\title{
Ezrin activation in vitro: \\ Investigation of ezrin's conformation and the interaction between ezrin and F-actin
}

\author{
Dissertation \\ for the award of the degree \\ Doctor rerum naturalium \\ Division of Mathematics and Natural Sciences \\ of the Georg-August-Universität Göttingen
}

\author{
submitted by \\ Julia Anna Braunger \\ from Biberach a. d. Riß
}

Göttingen 2013 
MEMBERS OF THE THESIS COMMITTEE:

Prof. Dr. Claudia Steinem (Reviewer),

Institute for Organic and Biomolecular Chemistry,

Georg-August-Universität Göttingen

Prof. Dr. Sarah Köster (Reviewer),

Institute for X-Ray Physics,

Georg-August-Universität Göttingen

Dr. Iwan Schaap,

Third Institute of Physics,

Georg-August-Universität Göttingen

DATE OF ORAL EXAMINATION:

21 June 2013 


\section{DECLARATION}

I, Julia Anna Braunger, hereby certify that my doctoral thesis entitled "Ezrin activation in vitro: Investigation of ezrin's conformation and the interaction between ezrin and F-actin" has been written independently and with no other sources and aids than quoted.

Göttingen, 2013

Julia Anna Braunger 

Für meine Familie 

ABSTRACT The function of ezrin, a member of the ezrin-radixin-moesin (ERM) protein family, is to regulate the cell membrane architecture within the context of fundamental biological processes by linking the membrane and the actin cytoskeleton. In the inactive state, ezrin is conformationally masked by self-association of $\mathrm{N}$ and $C$-terminal domains. Ezrin activation is thought to rely on a conformational change induced by binding to $\mathrm{L}-\alpha$-phosphatidylinositol-4,5-bisphosphate $\left(\mathrm{PIP}_{2}\right)$ and followed by phosphorylation of a conserved threonine $(\mathrm{T})$ residue, thus rendering the binding site for filamentous actin (F-actin) accessible. However, the contribution of the individual activation factor is still controversially discussed. Moreover, several lines of evidence indicate that $\mathrm{PIP}_{2}$ binding might be sufficient to establish a stable interaction between ezrin and F-actin

In the first part of this work, different strategies for the preparation of $\mathrm{PIP}_{2^{-}}$ containing solid-supported lipid bilayers (SLBs) are presented overcoming often encountered spreading issues owing to the multiple negative charges of $\mathrm{PIP}_{2}$. Reflectometric interference spectroscopy (RIfS) and fluorescence microscopy were employed to verify the SLB formation. With regard to reliable formation of SLBs containing high $\mathrm{PIP}_{2}$ fractions and subsequent ezrin binding to control the accessibility of $\mathrm{PIP}_{2}$, only spreading at $\mathrm{pH} 4.8$ gave satisfactory results, whereas calcium ions or increased ionic strength revealed to be not suitable. Solid-supported hybrid membranes, prepared by spreading of $\mathrm{PIP}_{2}$-containing vesicles on hydrophobically functionalized substrates, were characterized by comparably high lateral $\mathrm{PIP}_{2}$ mobility $\left(3 \mu \mathrm{m}^{2} / \mathrm{s}\right.$, mobile fraction of $\left.98 \%\right)$ in fluorescence recovery after photobleaching (FRAP) experiments using a fluorescent $\mathrm{PIP}_{2}$ analogue as tracer. Binding of ezrin to $\mathrm{PIP}_{2}$ effectively reduced its diffusion, implicating that ezrin is able to maintain $\mathrm{PIP}_{2}$ clusters within a cellular context.

The main part of this thesis is dedicated to investigating to what extent $\mathrm{PIP}_{2}$ and phosphorylation contribute to ezrin activation regarding on the one hand a possible height change of ezrin aggregates on SLBs due to the conformational change and on the other hand the strength of its interaction with F-actin. The influence of phosphorylation was assessed by using three ezrin variants, namely ezrin wildtype, ezrin T567A (nonphosphorylatable), and ezrin T567D (pseudophosphorylated). The influence of $\mathrm{PIP}_{2}$ on the activation was addressed by using two different ezrin immobilization strategies, either nickel nitrilotriacetic acid (NTA-Ni)or $\mathrm{PIP}_{2}$-mediated. Only the combination of $\mathrm{PIP}_{2}$ binding and phosphorylation led to a significantly lower protein height level on the SLB as determined by RIfS and atomic force microscopy imaging. By colloidal probe microscopy (CPM), it was found that $\mathrm{PIP}_{2}$ is sufficient to significantly enhance the interaction between ezrin and F-actin in terms of increased surface adhesion energies, whereas phosphorylation contributes to a minor extent. Notably, the adhesion forces remained almost unchanged. From these findings, we inferred that ezrin activation generates rather many weak than few strong bonds between ezrin and F-actin, forming a stable multivalent interaction. 
zusammenfassung Ezrin, ein Mitglied der Ezrin-Radixin-Moesin (ERM)-Proteinfamilie, ermöglicht eine dynamische Verknüpfung von Membran und Aktinzytoskelett und ist somit an grundlegenden biologischen Prozessen beteiligt. Ezrin wird durch intramolekularer Selbstassoziation in seiner Aktivität konformell reguliert. Als möglicher Aktivierungsmechanismus wird ein Konformationswechsel diskutiert, der durch Bindung an L- $\alpha$-Phosphatidylinositol-4,5-bisphosphat ( $\left.\mathrm{PIP}_{2}\right)$ und anschließender Phosphorylierung eines spezifischen Threoninrestes induziert wird. Allerdings wird auch postuliert, dass $\mathrm{PIP}_{2}$-Bindung als alleiniger aktivierender Faktor ausreichend ist.

Im ersten Teil dieser Arbeit wurden unterschiedliche Strategien für die Präparation $\mathrm{PIP}_{2}$-haltiger festkörperunterstützter Lipiddoppelschichten (SLBs) entwickelt, welche aufgrund der mehrfach negativen Ladung von $\mathrm{PIP}_{2}$ erschwert ist. Reflektometrische Interferenzspektroskopie (RIfS) und Fluoreszenzmikroskopie wurden eingesetzt um die SLB-Ausbildung zu überprüfen. Spreiten unilamellarer Vesikel in wässrigen Lösungen bei $\mathrm{pH} 4.8$ erwies sich für die Präparation $\mathrm{PIP}_{2}$-haltiger SLBs als geeignet, wohingegen die Verwendung von $\mathrm{Ca}^{2+}$ oder erhöhte Ionenstärke zu inhomogener $\mathrm{PIP}_{2}$-Verteilung führte. Festkörperunterstützte, $\mathrm{PIP}_{2}$-haltige Hybridmembranen wurden im Hinblick auf fluorescence recovery after photobleaching Experimente etabliert, wobei fluoreszenzmarkiertes $\mathrm{PIP}_{2}$ eingesetzt wurde. Es konnte gezeigt werden, dass die Bindung von Ezrin an $\mathrm{PIP}_{2}$ zur Reduzierung der lateralen Mobilität von $\mathrm{PIP}_{2}$ führt, was im zellulären Kontext ein Hinweis auf die Fähigkeit von Ezrin $\mathrm{PIP}_{2}$-Aggregate aufrechtzuerhalten ist.

Der Hauptteil dieser Arbeit adressiert die Frage, in welchem Ausmaß PIP 2 und Phosphorylierung zur Aktivierung von Ezrin beitragen. Zum einen wurde der Aspekt einer möglichen, mit dem Konformationswechsel einhergehenden Änderung der Höhe von Proteinmonoschichten auf SLBs untersucht. Zum anderen wurde die Stärke der Interaktion von Ezrin und F-Aktin quantifiziert. Der Einfluss der Phosphorylierung wurde durch Verwendung dreier Ezrinvarianten - Ezrin Wildtyp, Ezrin T567A (nicht-phosphorylierbar) und Ezrin T567D (pseudophosphoryliert) untersucht. Die Höhe der Proteinschichten auf SLBs wurde mittels RIfS und AFM (atomic force microscopy) bestimmt. Der Einfluss von $\mathrm{PIP}_{2}$ wurde durch Vergleich von $\mathrm{PIP}_{2}$ - oder Nickel-Nitrilotriessigsäure (NTA-Ni)-vermittelter Bindung der Proteine untersucht. Die Kombination von $\mathrm{PIP}_{2}$ und Phosphorylierung führte zu einer signifikanten Verringerung der detektierten Höhe der Proteinmonoschicht. Mittels colloidal probe microscopy (CPM), konnte gezeigt werden, dass $\mathrm{PIP}_{2}$ zu einer deutlich erhöhten Interaktion zwischen Ezrin und F-Aktin führt, was anhand vergrößerter Adhäsionsenergien gezeigt werden konnte. Der Einfluss der Phosphorylierung war hingegen geringer im Hinblick auf eine Verstärkung der Ezrin-F-Aktin-Interaktion. Es konnte gezeigt werden, dass die Aktivierung von Ezrin nicht mit einer signifikanten Erhöhung der Adhäsionskraft einer einzelnen Bindung zwischen Ezrin und F-Aktin einhergeht, woraus geschlossen werden kann, dass die Stärke der Ezrin-F-Aktin-Interaktion auf dem Zusammenwirken vieler schwacher Einzelbindungen basiert. 
CONTENTS

GENERAL INTRODUCTION

2 OVERVIEW AND SCOPE OF THESIS 5

3 METHODS AND MATERIALS $\quad 7$

3.1 Biochemical methods . . . . . . . . . . . . . . 7

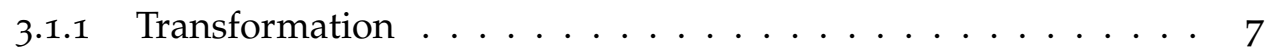

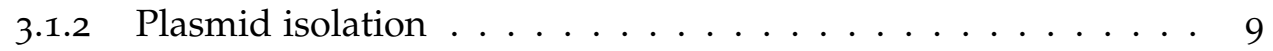

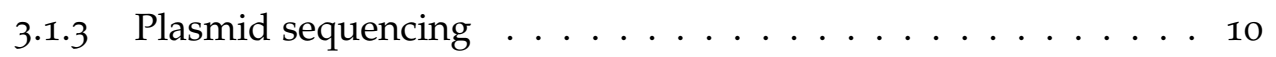

3.1.4 Heterologous protein expression ............. 10

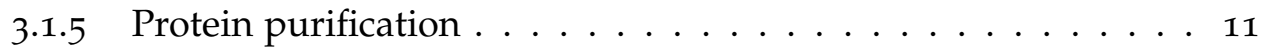

3.2 Surface functionalization . . . . . . . . . . . . . . . 14

3.2.1 Self-assembled monolayer formation .......... 15

3.2.2 Solid-supported membrane formation . . . . . . . . . . 16

3.3 Biophysical techniques . . . . . . . . . . . . . . 17

3.3.1 Atomic force microscopy ................... 17

3.3.2 Confocal laser scanning microscopy . . . . . . . . . . 26

3.3.3 Direct optical sensing techniques . . . . . . . . . . . 29

3.3.4 Scanning electron microscopy . . . . . . . . . . . 34

4 HOW MEMBRANE PREPARATION INFLUENCES PIP2 35

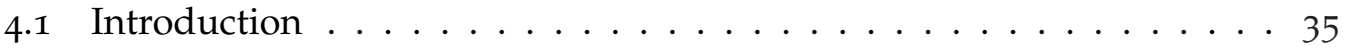

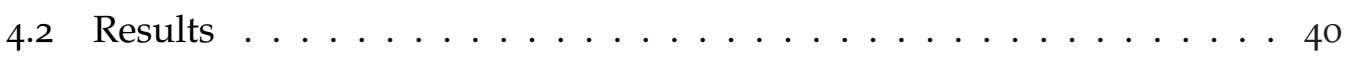

4.2.1 Spreading of $\mathrm{PIP}_{2}$-containing POPC vesicles $\left(8 \mathrm{~mol} \% \mathrm{PIP}_{2}\right)$ on silicon substrates and subsequent ezrin binding . . . . . . . 40

4.2.2 Lateral mobility of Bodipy TMR-PIP ${ }_{2}$ within solid-supported

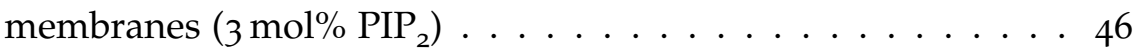

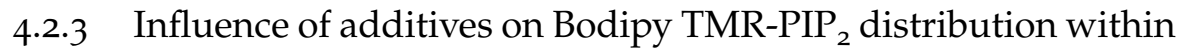
solid-supported hybrid membranes $\left(3 \mathrm{~mol}_{\%} \mathrm{PIP}_{2}\right)$. . . . . . . . 49

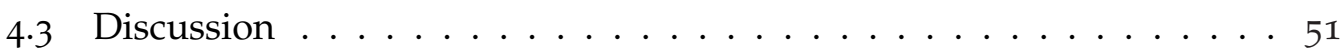

4.3.1 Influence of spreading conditions on solid-supported lipid bilayers $\left(8 \mathrm{~mol}_{\mathrm{o}} \mathrm{PIP}_{2}\right)$. . . . . . . . . . . . . 51

4.3.2 $\mathrm{PIP}_{2}$ mobility within solid-supported membranes $\left(3 \mathrm{~mol} \% \mathrm{PIP}_{2}\right) 56$

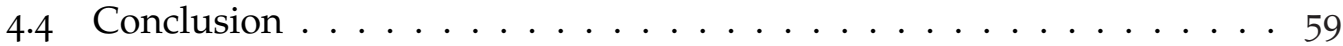

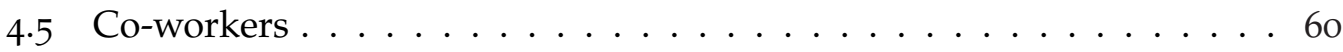

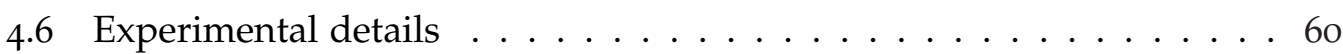

5 ON THE TRAIL OF THE CONFORMATIONAL CHANGE UPON EZRIN AC-

$\begin{array}{ll}\text { TIVATION } & 61\end{array}$ 


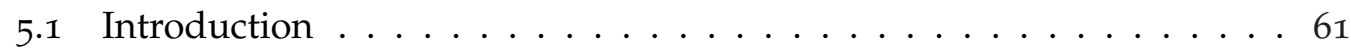

5.2 Results . . . . . . . . . . . . . . . . . . 65 65

5.2.1 Transformation and isolation of ezrin . . . . . . . . . 65

5.2.2 Exploring ezrin aggregates on solid-supported lipid bilayers by RIfS . . . . . . . . . . . . . . . . . . . . 666

5.2.3 Evaluating height level and morphology of ezrin aggregates by $\mathrm{AFM} \ldots \ldots \ldots \ldots \ldots \ldots \ldots \ldots$

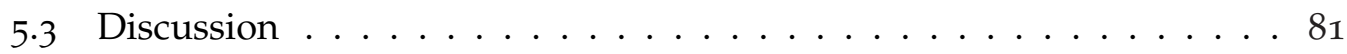

5.3.1 Tracing the conformational change by evaluating ezrin height

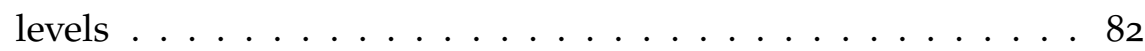

5.3.2 Pre-organization of $\mathrm{PIP}_{2}$ influences ezrin cluster morphology . 86

5.3.3 Drawing conclusions from the degree of protein surface coverage ..................... 87

5.4 Conclusion . . . . . . . . . . . . . . . . . . . . . . . . . . . 89

5.5 Co-workers . . . . . . . . . . . . . . . . . 90

5.6 Experimental details . . . . . . . . . . . . . . 90

5.7 Appendix . . . . . . . . . . . . . . . . . . . . 92

6 MODEL SYSTEM EXPLORING THE FORCES AT THE MEMBRANE CYTOSKELE-

TON INTERFACE 95

6.1 Introduction . . . . . . . . . . . . . . . . . 95

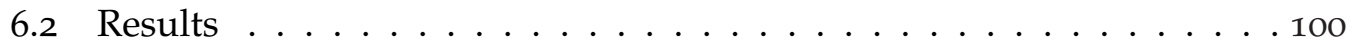

6.2.1 Surface functionalization strategy and setup . . . . . . . 100

6.2.2 Measuring the forces between F-actin and ezrin as a function of its activation . . . . . . . . . . . . . . 104

6.2.3 Colloidal probe microscopy revealing differences and similarities in adhesion between F-actin and ezrin as a function of its

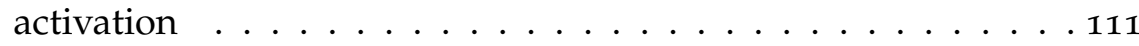

6.2.4 Low adhesion forces in CPM experiments - a comparison with single molecule experiments . . . . . . . . . . . . 120

6.3 Discussion . . . . . . . . . . . . . . . . . . . . . 122

6.3.1 NTA-Ni-His 6 and electrostatics overcome the ezrin F-actin connection . . . . . . . . . . . . . . . . . . .123

6.3.2 Activation of ezrin: Increasing surface adhesion energies instead of adhesion forces . . . . . . . . . . . . . 123

6.3.3 Ensemble versus single molecule measurement: Similar adhesion forces in both SMFM and CPM experiments . . . . . . 126

6.3.4 Molecular force spectroscopy: Dependence of mean adhesion force on loading rate . . . . . . . . . . . . . 133

6.4 The force response to ezrin activation - Conclusion $\ldots . \ldots 134$ 
6.5 Co-workers . . . . . . . . . . . . . . . . . . . . . . 135

6.6 Experimental details $\ldots \ldots \ldots . \ldots \ldots$

$\begin{array}{lll}7 & \text { SUMMARY OF THESIS } & 139\end{array}$

$\begin{array}{ll}\text { A APPENDIX } & 143\end{array}$

A.1 Abbreviations . . . . . . . . . . . . . . . . 143

A.2 Chemicals . . . . . . . . . . . . . . . . . . 145

A.3 Hardware . . . . . . . . . . . . . . . . . . 1446

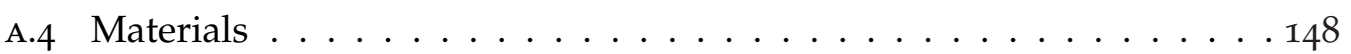

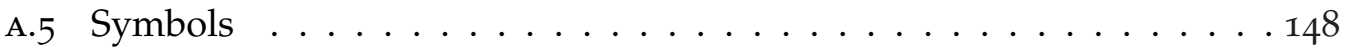

$\begin{array}{lr}\text { BIBLIOGRAPHY } & 153\end{array}$ 



\section{GENERAL INTRODUCTION}

Fundamental cellular processes occurring at the membrane-cytoskeleton interface, including cell motility, adhesion, endo- and exocytosis, rely on a finely balanced linkage between the plasma membrane and the subjacent actin cytoskeleton. Linker proteins such as the members of the ezrin-radixin-moesin (ERM) protein family provide such a linkage. ${ }^{[1]}$ ERM proteins are highly conserved in their sequence throughout evolution, giving rise to the assumption of redundant functions among ERM proteins. ${ }^{[2,3]}$ However, the expression of the ERM proteins in adult mammals revealed to be rather tissue-specific. Ezrin is predominantly found in epithelial cells, radixin in hepatocytes, whereas moesin is mainly expressed in the endothelium. ${ }^{[4]}$ The generation of ezrin-deficient mice unveiled the critical role of this ERM protein in intestinal epithelial cells. Wildtype intestinal epithelial cells were characterized by uniform, densely arranged brush border microvilli (figure 1.1 A), whereas ezrin deficiency led to severe apical defects generating misoriented, thickened microvilli (figure 1.1 B). ${ }^{[5]}$
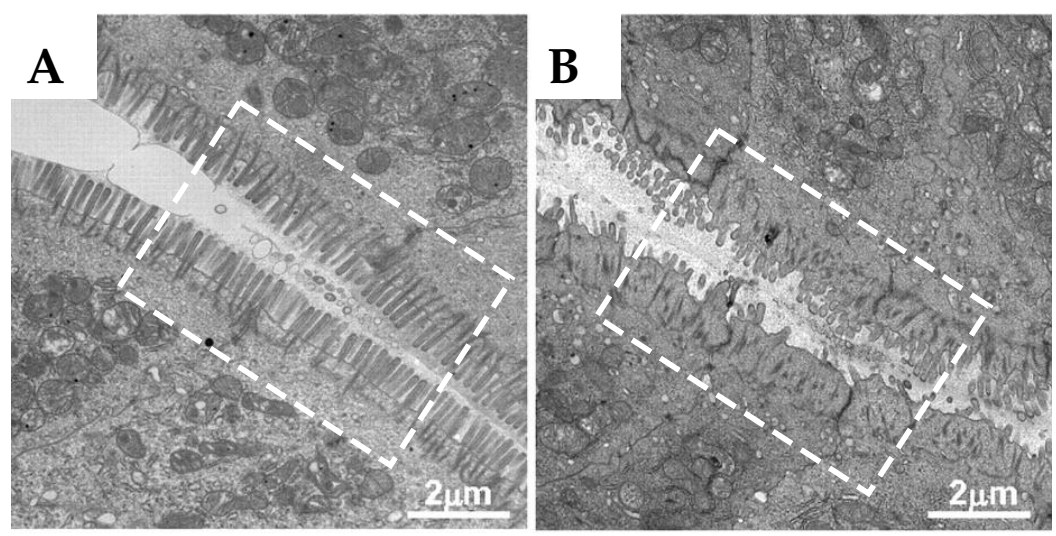

Figure 1.1: Transmission electron micrographs showing the influence of ezrin on brush border microvilli (white dashed box) at the apical surface of intestinal epithelial cells. Wildtype intestinal epithelial cells are characterized by uniform, densely arranged brush border microvilli (A). Severe apical defects arise in ezrin deficient intestinal epithelial cells resulting in misoriented, thickened microvilli (B). 1100o $\times$ magnification in panels A and B. Reproduced from Casaletto et al. [5]

In the light of the continuous dynamic interplay between the plasma membrane and the actin cytoskeleton, a balanced regulation of the ERM linker function is required. Two findings revealed that ERM proteins are conformationally regulated by head-to-tail folding. (1) A binding site for filamentous actin (F-actin) was identified in the last 34 amino acids of ezrin, ${ }^{6]}$ and (2) this F-actin binding site was found 
to be masked due to the strong association tendency between $C$-terminal and $N$ terminal domains of ezrin. ${ }^{[7]}$ First insights into the regulation of ERM proteins on a molecular level were provided by the finding that moesin is phosphorylated at a specific threonine $(\mathrm{T})$ residue during platelet activation, leading to colocalization of phosphorylated moesin and F-actin. ${ }^{[8]}$ This conserved threonine residue within the C-terminal domain of ERM proteins was identified for ezrin, radixin, and moesin $-\mathrm{T}_{5} 67, \mathrm{~T}_{5} 64$, and $\mathrm{T}_{55} 8$, respectively - as target for phosphorylation by Rho-kinase and protein kinase $C \Theta .{ }^{[8,9]}$ At the same time, a binding site for the phosphoinositide L- $\alpha$-phosphatidylinositol-4,5-bisphosphate $\left(\mathrm{PIP}_{2}\right)$ was identified within the $N$-terminal part of ezrin. ${ }^{[10]}$ First, the role of $\mathrm{PIP}_{2}$ was confined to membrane attachment of ERM proteins.

The phosphorylation of the specific threonine residue was considered long time to be the only activation factor of ERM proteins. However, lines of evidence accumulate that the role of $\mathrm{PIP}_{2}$ is not only restricted to provide membrane attachment of ERM proteins, but also a functional role of $\mathrm{PIP}_{2}$ in their activation mechanism is suggested. ${ }^{[1-13]}$ Roch et al. investigated the contribution of $\mathrm{PIP}_{2}$ and phosphorylation to the activation of moesin during Drosophila development, revealing intriguingly differential roles of $\mathrm{PIP}_{2}$ and phosphorylation. ${ }^{[13]}$ Moesin (Moe) deficient flies were characterized by abnormally rough eyes and small crumpled wings in comparison to wildtype flies (figure 1.2 A, B). Different GFP-labeled moesin variants were evaluated in vivo regarding their ability to substitute for endogenous moesin during Drosophila development.

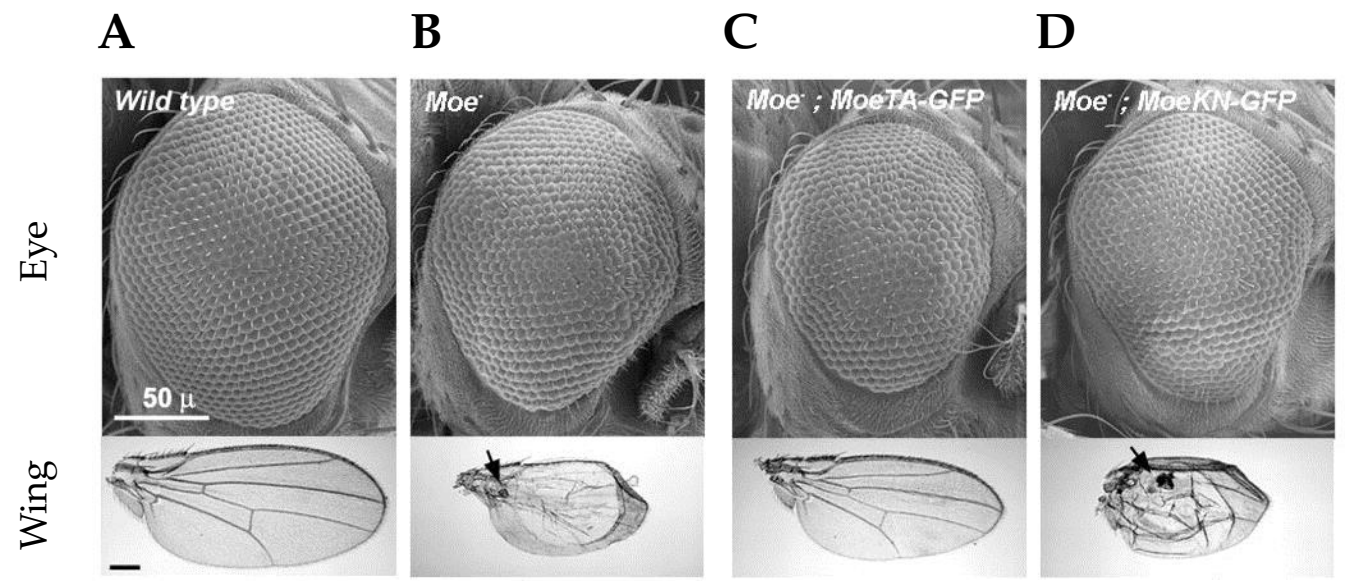

Figure 1.2: Activity tests of different Moe variants in vivo monitoring their ability to substitute for the endogenous product during fly development. Scanning electron micrographs of adult eyes and pictures of wings were taken from the wildtype fly (A) and moesindeficient flies (B-D). Moesin deficiency leads to the development of reduced rough eyes and small crumpled wings (B). Expression of the moesin wildtype analogue MoeWT-GFP rescues both eye and wing phenotypes (not shown). The nonphosphorylatable MoeTAGFP mutant does not rescue the eye but the wing phenotype (C). The mutant deficient in PIP $_{2}$ binding, namely MoeKN-GFP, fails in rescuing both phenotypes (D). Scale bars: $50 \mu \mathrm{m}$ top panels, $250 \mu \mathrm{m}$ bottom panels. Reproduced from Roch et al. ${ }^{[13]}$ 
The expression of the moesin wildtype analogue MoeWT-GFP was able to rescue both the wing and eye phenotypes of moesin deficient flies (not shown). Notably, the nonphosphorylatable moesin variant MoeTA-GFP did not favor normal eye development, however, it was able to rescue the wing phenotype (figure 1.2 C). In light of the long assumed prerequisite of phosphorylation for activation of ERM proteins, this is a striking result. In addition, the moesin variant abolished in PIP $_{2}$ binding, namely MoeKN-GFP, was neither able to rescue the eye nor the wing phenotype, thus emphasizing the pivotal role of $\mathrm{PIP}_{2}$ regarding moesin activation in Drosophila (figure 1.2 D). This is corroborated by Yonemura et al. showing that threonine phosphorylation is not necessarily required for ERM activation. ${ }^{[11]}$ Based on these findings, a two-step model was proposed for ERM activation comprising recruitment to the membrane and activation by $\mathrm{PIP}_{2}$ followed by phosphorylation of the conserved threonine residue in the $C$-terminal domain providing a fine-tune mechanism to further stabilize the interaction between F-actin and ERM proteins. ${ }^{[11,13]}$

The interpretation of an observed effect caused by a specific activation factor is often hampered due to the interwoven regulation mechanisms within the cell. Therefore, model systems are suited to reduce the cellular complexity, allowing the investigation of individual interactions in a well-defined environment. An indirect measure of ERM activation is the capability of ERM proteins to bind to F-actin as the conformational change of ERM proteins upon activation is prerequisite for the release of the F-actin binding site. In a recent confocal laser scanning microscopy (CLSM) based assay to probe the F-actin binding capability of ezrin, we aimed to assess the individual contributions of $\mathrm{PIP}_{2}$ binding and phosphorylation by using an artificial membrane system (figure 1.3). ${ }^{[14]}$ We were able to show that ezrin wildtype (wt) bound via $\mathrm{PIP}_{2}$ to a solid-supported lipid bilayer (SLB) led to a significantly higher F-actin surface coverage than ezrin wildtype bound through a $6 \times$ histidine $\left(\mathrm{His}_{6}\right)$ tag to a SLB displaying nickel nitrilotriacetic acid (NTA-Ni) head groups. By using pseudophosphorylated ezrin $\mathrm{T}_{5} 67 \mathrm{D}$, we mimicked the activating influence of phosphorylation. From the significantly increased F-actin surface coverage in case of ezrin $\mathrm{T}_{5} 67 \mathrm{D}$ bound via $\mathrm{PIP}_{2}$, we concluded a synergism between phosphorylation and $\mathrm{PIP}_{2}$. 

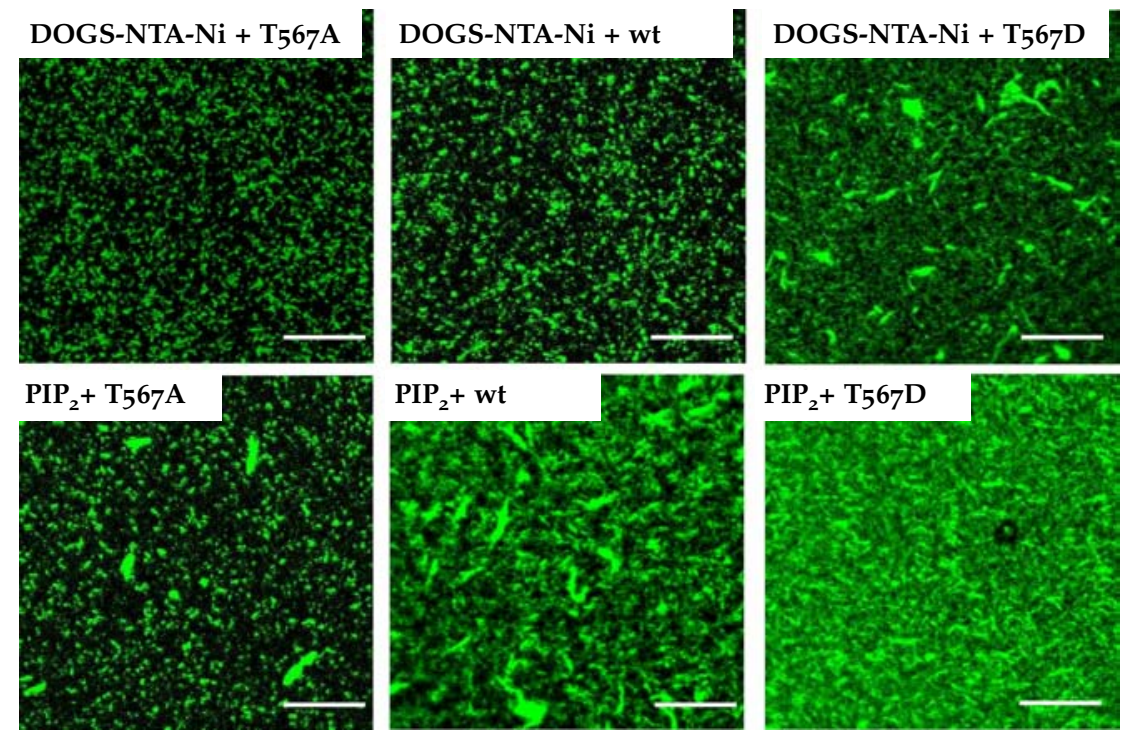

Figure 1.3: F-actin surface coverage depending on ezrin activation. CLSM images of AlexaFluor488-phalloidin labeled actin bound to ezrin on DOGS-NTA-Ni (10\%) doped solid-supported lipid bilayers (SLBs) or $\mathrm{PIP}_{2}$ (10\% doped SLBs on silicon substrates using ezrin T567A, ezrin wildtype (wt), and ezrin $\mathrm{T}_{56} 6_{7} \mathrm{D}$ as indicated. Scale bars: $10 \mu \mathrm{m}$. Reproduced from Bosk et al. ${ }^{[14]}$

The F-actin cytoskeleton is the major force bearing structure within the cell, providing mechanical stability and sustaining forces. Evidently, the bond between F-actin and ezrin is exposed to force as well. This gives rise to the question how the interaction between F-actin and ezrin is affected by the activation factors PIP $_{2}$ and phosphorylation. First insights were obtained in colloidal probe experiments showing that the interaction between F-actin and ezrin wildtype bound via $\mathrm{PIP}_{2}$ to SLBs was characterized by significantly larger adhesion forces and energies as compared to that between F-actin and ezrin wildtype adsorbed via its $\mathrm{His}_{6}$-tag to a SLB displaying NTA-Ni head groups. ${ }^{\left[{ }^{15}\right]}$ So far, a comprehensive characterization covering the individual contributions of $\mathrm{PIP}_{2}$ and phosphorylation with regard to possible differences in adhesion forces and energies between F-actin and ezrin is not available. 
The main objectives of this thesis were (1) the investigation of the conformational change of ezrin due to its activation, i.e., L- $\alpha$-phosphatidylinositol-4,5-bisphosphate $\left(\mathrm{PIP}_{2}\right)$ binding and phosphorylation, and (2) the development of a model system allowing the thorough characterization of the F-actin (filamentous actin) binding capability of ezrin as a function of its activation.

Chapter 4 focuses on the preparation of $\mathrm{PIP}_{2}$-containing solid-supported lipid bilayers (SLBs) and hybrid membranes (SHMs). Owing to the multiple negative charges of $\mathrm{PIP}_{2}$, the preparation of SLBs containing high $\mathrm{PIP}_{2}$ fractions is hampered. The spreading process was monitored by reflectometric interference spectroscopy (RIfS) and confocal laser scanning microscopy (CLSM). To evaluate the influence of different spreading protocols on the accessibility of $\mathrm{PIP}_{2}$, the $\mathrm{PIP}_{2}$ binding protein ezrin was added after membrane formation and visualized by RIfS and CLSM. The influence of $\mathrm{PIP}_{2}$ binding agents - calcium ions and ezrin - was evaluated in fluorescence recovery after photobleaching (FRAP) experiments in terms of diffusion coefficients and immobile fractions.

In chapter 5, the question was addressed whether the conformational change of ezrin due to activation can be investigated by RIfS and atomic force microscopy (AFM) imaging in terms of changes in protein height levels and surface coverages on SLBs. To map the influence of the individual activation step, ezrin wildtype and two mutants - nonphosphorylatable and pseudophosphorylated variants - were employed. Moreover, membrane attachment of the proteins was either achieved by binding to the natural receptor lipid PIP 2 or via its $N$-terminal $6 \times$ histidine $\left(\mathrm{His}_{6}\right.$ ) tag to a NTA-Ni-terminated lipid to evaluate the influence of PIP ${ }_{2}$.

The main part of this thesis deals with the thorough investigation of the interaction between ezrin and F-actin by force measurements described in chapter 6 . Colloidal probe microscopy (CPM) was used to analyze the strength of the interaction between F-actin and ezrin depending on its activation state with respect to adhesion forces and surface adhesion energies. Similar to chapter 5, ezrin wildtype and mutants were used in combination with $\mathrm{PIP}_{2}$ to assess the individual contributions of the activation factors in the context of the F-actin binding capability of ezrin. 

METHODS AND MATERIALS

\subsection{BIOCHEMICAL METHODS}

Bacterial expression systems are widely used for heterologous protein expression due to their capability to grow fast and at high density. In particular, the well characterized genetics as well as the large number of available cloning vectors and mutant host strains make the Escherichia coli (E. coli) bacterium an excellent choice for the expression of protein if no posttranslational modifications, such as glycosylation, are required. A widely used promoter system in E. coli is the $\mathrm{T}_{7}$ RNA polymerase system. Based on this development by Studier and colleagues, the pET vector series by Novagen has been established. ${ }^{[16]}$ The most commonly used host strain in combination with the pET system is BL21, which is available with a prophage $\left(\lambda \mathrm{DE}_{3}\right)$ encoding the $\mathrm{T}_{7}$ RNA polymerase under the control of the lac $\mathrm{UV}_{5}$ promoter. This promoter is induced by the non-hydrolyzable isopropyl $\beta$ D-1-thiogalactopyranoside (IPTG). However, there is basal expression of $\mathrm{T}_{7}$ RNA polymerase even in uninduced cells, which poses a problem in case of toxic target proteins. More stringent control is achieved using a host carrying the vector pLysS. This plasmid expresses $\mathrm{T}_{7}$ lysozyme, a natural $\mathrm{T}_{7}$ RNA polymerase inhibitor, thus preventing the transcription of target genes in uninduced cells. Most E. coli strains can be used to propagate a plasmid, although the characteristics of the particular strain have to be taken into account. The level of endonuclease activity, methylation and growth characteristics may strongly influence the quality of DNA. The E. coli strain BL21(DE3)pLysS was used as production host, while the E. coli strain TOP1o is ideal for plasmid propagation. ${ }^{[17,18]}$

All steps sensitive to microbiological contamination, such as transformation, cell growth and cultivation were carried out under aseptic conditions using a laminarflow clean bench. All glassware, tubes, culture media and distilled water were autoclaved at $121{ }^{\circ} \mathrm{C}$ for $20 \mathrm{~min}$ to sterilize them and to avoid contamination.

\subsubsection{Transformation}

Chemically competent cells (One Shot ${ }^{\circledR}$ cells, table 3.1) were purchased from Life Technologies (Darmstadt, Germany). The cells are calcium chloride treated to facilitate the uptake of exogenous genetic material upon a brief heat-shock. 
Table 3.1: Chemically competent $E$. coli cells and corresponding genotypes.

\begin{tabular}{|c|c|}
\hline Strain & Genotype \\
\hline BL21(DE3)pLysS & F- ompT gal dcm lon hsdSB(rB- mB-) $\lambda\left(\mathrm{DE}_{3}\right) \mathrm{pLysS}\left(\mathrm{cm}^{\mathrm{R}}\right)$ \\
\hline TOPio & 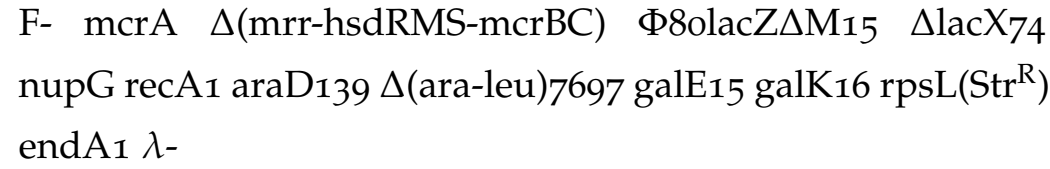 \\
\hline
\end{tabular}

The pET28a(+) vector (Merck Millipore, Darmstadt, Germany) with the insertion of either human cDNA of ezrin wildtype, ezrin $T_{56} 6 \mathrm{~A}$ or ezrin $\mathrm{T}_{5} 67 \mathrm{D}$ (table 3.2) was provided by Prof. Dr. Volker Gerke (ZMBE, Münster).

Table 3.2: Plasmids.

\begin{tabular}{llll}
\hline Plasmid name & Insert & Vector & Resistance \\
\hline pEzrinWT & full human ezrin cDNA & pET28a $(+)$ & kan \\
\hline pEzrinT567A & full human ezrin cDNA, substitution & pET28a(+) & kan \\
& T567A $^{2}$ & & \\
\hline pEzrinT567D & full human ezrin cDNA, substitution & pET28a $(+)$ & kan \\
& T567D & & \\
\hline
\end{tabular}

The vials containing the plasmids were briefly centrifuged and placed on ice until use. For each transformation, one vial $(50 \mu l)$ of One Shot ${ }^{\circledR}$ cells was thawed on ice. 8ong of the corresponding plasmid was pipetted to a vial of competent cells and mixed gently. The transformation mixture was placed on ice for $30 \mathrm{~min}$. Thereafter, the vials were incubated for exactly $30 \mathrm{~s}$ in a water bath at $42^{\circ} \mathrm{C}$. Then, they were removed from the water bath and cooled down on ice. To allow for replication of the desired plasmid, $250 \mu \mathrm{l}$ of pre-warmed SOC medium $\left(37^{\circ} \mathrm{C}\right)$ were added to each transformation mixture. The vials were incubated for $1 \mathrm{~h}$ in a shaking incubator $\left(225 \mathrm{rpm}, 37^{\circ} \mathrm{C}\right) .50 \mu \mathrm{l}$ of the transformation mixture were spread on one half of a pre-warmed, kanamycin containing agar plate $\left(60 \mu \mathrm{g} / \mathrm{ml}, 37^{\circ} \mathrm{C}\right)$. The remaining cells in the vial were pelleted and most of the supernatant was discarded. Resuspending the cells with approximately $50 \mu l$ of the remaining supernatant yielded a suspension of high cell concentration, which was spread on the other half of the agar plate. The inoculated agar plates were inverted and incubated overnight at $37^{\circ} \mathrm{C}$. After successful transformation, clones were picked the next day for overnight cultures at $37^{\circ} \mathrm{C}$ in LB medium containing the appropriate antibiotics, that is, kanamycin $(60 \mu \mathrm{g} / \mathrm{ml})$ for cells containing the pET28a(+) vector 
and additionally chloramphenicol $(34 \mu \mathrm{g} / \mathrm{ml})$ for cells carrying the pLysS plasmid. Glycerol stocks $(1: 1(v / v))$ were prepared from overnight cultures, snap-frozen in liquid nitrogen and stored at $-80^{\circ} \mathrm{C}$.

Table 3.3: Media used for transformation.

\begin{tabular}{|c|c|}
\hline Name & Composition \\
\hline LB medium & $\begin{array}{l}\text { 10 } \mathrm{g} / 1 \text { tryptone, } 5 \mathrm{~g} / 1 \text { yeast extract, } 10 \mathrm{~g} / 1 \\
\mathrm{NaCl}, \mathrm{pH} 7.4\end{array}$ \\
\hline SOC medium & $\begin{array}{l}20 \mathrm{~g} / 1 \text { tryptone, } 5 \mathrm{~g} / 1 \text { yeast extract, } 10 \mathrm{~mm} \\
\mathrm{NaCl}, 2.5 \mathrm{~mm} \mathrm{KCl}, 10 \mathrm{mM} \mathrm{MgCl}_{2} \text {, } 10 \mathrm{~mm} \\
\mathrm{MgSO}_{4}, 20 \mathrm{~mm} \text { glucose }\end{array}$ \\
\hline LB agar plates & $\begin{array}{l}10 \mathrm{~g} / 1 \text { tryptone, } 5 \mathrm{~g} / 1 \text { yeast extract, } 10 \mathrm{~g} / 1 \\
\mathrm{NaCl}, 18 \mathrm{~g} / \mathrm{l} \text { agar, } \mathrm{pH} 7.4\end{array}$ \\
\hline Kanamycin stock solution & $30 \mathrm{mg} / 1$ in ultrapure water \\
\hline Chloramphenicol stock solution & $34 \mathrm{mg} / 1$ in ethanol p.a. \\
\hline
\end{tabular}

\subsubsection{Plasmid isolation}

Alkaline Lysis was employed to isolate plasmid DNA. Briefly, a single colony was transferred into $10 \mathrm{ml} \mathrm{LB}$ medium, containing kanamycin $(30 \mu \mathrm{g} / \mathrm{ml})$. The culture was incubated at $37^{\circ} \mathrm{C}$ vigorously shaking overnight. $5 \mathrm{ml}$ of the cell suspension were centrifuged to harvest the cells. After removal of the medium, the bacterial pellet was resuspended in $200 \mu \mathrm{l}$ of ice-cold Solution I. $400 \mu \mathrm{l}$ of Solution II were added. The contents were mixed by inverting the tube 4-6 times. Subsequently, $300 \mu \mathrm{l}$ of Solution III were added and the tube was again inverted 4-6 times. Then, the tube was incubated for $5 \mathrm{~min}$ on ice to allow for the renaturation of plasmid DNA. The tube was centrifuged for $5 \mathrm{~min}\left(12000 \times \mathrm{g}, 4{ }^{\circ} \mathrm{C}\right)$. Thereafter, the supernatant was transferred to a fresh tube. To precipitate the plasmid DNA, $750 \mu \mathrm{l}$ of ice-cold isopropanol were added and the tube was inverted several times. Spinningdown the mixture for $15 \mathrm{~min}\left(12000 \times \mathrm{g}, 4^{\circ} \mathrm{C}\right)$ yielded a white pellet. After removal of the supernatant, the pellet was rinsed in ice-cold $70 \%$ ethanol and air-dried for approximately $10 \mathrm{~min}$. The clean DNA pellet was resuspended in $20 \mu 1$ ultrapure water. The final concentration was determined via UV absorbance at $\lambda=260 \mathrm{~nm}$ measured with a NanoDrop spectrophotometer (Thermo Scientific, Wilmington, USA). 
Table 3.4: Solutions and buffers used for plasmid isolation.

\begin{tabular}{ll}
\hline Name & Composition \\
Solution I & $50 \mathrm{~mm}$ TRIS $/ \mathrm{HCl} \quad(\mathrm{pH} 8.0)$, 10 mM EDTA (pH 8.0), 10o mg $/ \mu l$ \\
& RNAse A \\
\hline Solution II & $0.2 \mathrm{M} \mathrm{NaOH}, \mathrm{I} \%(w / v)$ SDS \\
\hline Solution III & 3 M potassium acetate $\left(\mathrm{pH}_{5.5}\right)$ \\
\hline
\end{tabular}

\subsubsection{Plasmid sequencing}

The plasmid sequencing was carried out by Seqlab, Göttingen. The primers used to achieve full sequencing are listed in table 3.5. $\mathrm{T}_{7}$ promotor and terminator primers were purchased from Life Technologies (Darmstadt, Germany), while ezrin forward and reverse primers were synthesized by Sigma Aldrich (Steinheim, Germany).

Table 3.5: Primers used for plasmid sequencing.

\begin{tabular}{ll}
\hline Name & Sequence \\
\hline T7 promoter primer & $5^{\prime}$-TAATACGACTCACTATAGGG-3' \\
\hline Ezrin forward primer & $5^{\prime}$-GGAAATGTATGGAATCAAC-3' \\
\hline Ezrin reverse primer & $5^{\prime}$-CTCCTCATAGTCCTGCAG-3' \\
\hline T7 terminator primer & $5^{\prime}$-GCTAGTTATTGCTCAGCGG-3' \\
\hline
\end{tabular}

\subsubsection{Heterologous protein expression}

A single colony of E. coli BL21(DE3)pLysS containing the corresponding expression vector (table 3.2) was picked from a freshly streaked agar plate to inoculate an overnight culture (10 $\mathrm{ml}$ LB medium) supplemented with chloramphenicol $(34 \mu \mathrm{g} / \mathrm{ml})$ and kanamycin $(6 \mathrm{o} \mathrm{g} / \mathrm{ml})$. The culture was grown for $12 \mathrm{~h}$ to $14 \mathrm{~h}$ at $37^{\circ} \mathrm{C}$ vigorously shaking. The saturated culture was diluted into fresh LB medium (containing kanamycin $(60 \mu \mathrm{g} / \mathrm{ml})$ ) 1:50 and grown with shaking at $37^{\circ} \mathrm{C}$ to an optical density of 0.5 to 0.7 at $\lambda=600 \mathrm{~nm}$. Protein expression was induced by addition of IPTG ( $1 \mathrm{~mm}$ ). The culture was incubated for $3 \mathrm{~h}$ at $37^{\circ} \mathrm{C}$ with shaking. To stop protein expression, the cells were cooled down for $10 \mathrm{~min}$ on ice. The cells were harvested by centrifugation $\left(4000 \times \mathrm{g}, 20 \mathrm{~min}, 4{ }^{\circ} \mathrm{C}\right.$ ). After decanting the supernatant, the pellet was resuspended in ice-cold lysis buffer $(25 \mathrm{ml})$. To complete lysis, the suspension was sonicated on ice $(5 \times 30 \mathrm{~s}$, duty cycle $50 \%)$. The bacterial lysate was clarified by centrifugation (100 000 $\mathrm{g}, \mathrm{I} \mathrm{h}, 4^{\circ} \mathrm{C}$ ). 
Table 3.6: Solutions and buffers used for protein expression.

\begin{tabular}{ll}
\hline Name & Composition \\
\hline IPTG stock solution & 1 M IPTG in ultrapure water \\
\hline Lysis buffer & $40 \mathrm{mM} \mathrm{HEPES,} \mathrm{20} \mathrm{mM} \mathrm{imidazole,} \mathrm{300} \mathrm{mM} \mathrm{NaCl,} \mathrm{1} \mathrm{mM}$ \\
& EDTA, pH 7.4 \\
& immediately before use: 10 mM 2-mercaptoethanol, \\
& I tablet/50 ml lysate of complete EDTA-free protease in- \\
& hibitor cocktail (Roche Diagnostics, Mannheim) \\
\hline
\end{tabular}

\subsubsection{Protein purification}

The proteins ezrin wildtype, ezrin $\mathrm{T}_{56} 6 \mathrm{~A}$, and ezrin $\mathrm{T}_{56} 6 \mathrm{D}$ used in this work were expressed as $\mathrm{N}$-terminally hexahistidine-tagged $\left(\mathrm{His}_{6}\right)$ proteins. The $\mathrm{His}_{6}$-tag allows protein purification from the clarified bacterial lysate via nickel affinity chromatography. $4 \mathrm{ml}$ of the metal-chelating material nickel-nitrilotriacetic acid (NTA$\mathrm{Ni}$ ) agarose (Quiagen, Hilden, Germany) were pipetted into a purification column. The solution was allowed to settle down evenly by gravity flow yielding a column volume of $\sim 2 \mathrm{ml}$. The column was washed with 1o column volumes of ultrapure water and equilibrated with the same amount of equilibration buffer. The lysate was applied three times onto the column to assure that most of the protein was immobilized. Wash steps were performed with 1o column volumes of wash buffer I, and subsequently, with 1o column volumes of wash buffer II. Elution of purified protein was achieved with 9 column volumes of elution buffer. The eluate was collected in $1 \mathrm{ml}$ fractions.

Table 3.7: Buffers used for protein isolation.

\begin{tabular}{|c|c|}
\hline Name & Composition \\
\hline Equilibration buffer & $40 \mathrm{~mm}$ HEPES, $20 \mathrm{~mm}$ imidazole, $300 \mathrm{~mm} \mathrm{NaCl}, \mathrm{pH} 7 \cdot 4$ \\
\hline Wash buffer I & $\begin{array}{l}40 \mathrm{~mm} \text { HEPES, } 30 \mathrm{~mm} \text { imidazole, } 300 \mathrm{~mm} \mathrm{NaCl}, 1 \mathrm{~mm} \\
\mathrm{MgCl}_{2}, \mathrm{pH} 7.4 \\
\text { immediately before use: } 10 \mathrm{~mm} \text { 2-mercaptoethanol }\end{array}$ \\
\hline Wash buffer II & $\begin{array}{l}40 \mathrm{mM} \text { HEPES, } 50 \mathrm{~mm} \text { imidazole, } 150 \mathrm{~mm} \mathrm{NaCl}, 1 \mathrm{~mm} \\
\mathrm{MgCl}_{2}, \mathrm{pH} 7.4 \\
\text { immediately before use: } 10 \mathrm{~mm} \text { 2-mercaptoethanol }\end{array}$ \\
\hline Elution buffer & $20 \mathrm{~mm}$ TRIS/HCl, $250 \mathrm{~mm}$ imidazole, $50 \mathrm{~mm} \mathrm{KCl,} \mathrm{pH} 7.4$ \\
\hline
\end{tabular}

All protein purification steps were performed at $4{ }^{\circ} \mathrm{C}$. Bacterial lysate, wash and elution fractions were analyzed by SDS-PAGE (section 3.1.5.1). 


\subsubsection{SDS polyacrylamide gel electrophoresis}

Sodium dodecyl sulfate polyacrylamide gel electrophoresis (SDS-PAGE) allows the separation of proteins according to their electrophoretic mobility. A discontinuous polyacrylamide gel, consisting of stacking and resolving gel, is used as support medium. The anionic detergent SDS binds noncovalently to proteins and thus transfers an overall negative charge according to their molecular weight while masking inherent charges. SDS causes denaturation of proteins disrupting non-covalent interactions. The electrophoretic mobility of a protein in the polyacrylamide gel is determined by both, the charge-to-mass ratio of the protein and the pore size of the gel. ${ }^{[19]}$

In this work, the vertical dual gel electrophoresis system PerfectBlue ${ }^{\mathrm{TM}}$ Twin (PEQLAB Biotechnologie, Erlangen) was used for SDS PAGE of protein fractions obtained during protein isolation (section 3.1.5). Polyacrylamide gels were prepared by radical polymerization of acrylamide and bisacrylamide upon addition of the initiator ammonium peroxydisulfate (APS) and the stabilizer $N, N, N^{\prime}, N^{\prime}$-tetramethylethane-1,2-diamine (TEMED). The resolving gel (12.5\%, pH 6.8) was cast by mixing $4 \mathrm{ml}$ stock solution, $40 \mu \mathrm{l}$ APS, and $2 \mu$ TEMED. The solution was poured into the casting assembly, overlayed with isopropanol, and allowed to polymerize for $45 \mathrm{~min}$. To prepare the stacking gel $(5.2 \%$, $\mathrm{pH} 8.8)$, isopropanol was removed and $2 \mathrm{ml}$ stock solution, $20 \mu \mathrm{l}$ APS, and $2 \mu \mathrm{l}$ TEMED were added on top of the resolving gel. A comb was immediately placed into the stacking gel to form sample wells. After approximately $45 \mathrm{~min}$, the polymerization was finished and the casting assembly was placed in the outer buffer chamber. It was filled with electrophoresis buffer and the comb was removed. Samples for SDS PAGE were prepared according to the following procedure: From each fraction of interest $10 \mu 1$ were taken and the same volume of sample buffer was added. The mixture was heated in a thermomixer at $85^{\circ} \mathrm{C}$ (5 $\left.\mathrm{min}, 350 \mathrm{rpm}\right)$ to denature the proteins. After denaturation, the samples were pipetted into the wells. One well was loaded with low molecular weight markers (GE Healthcare, Freiburg, Germany). The gel electrophoresis system was connected to a power source applying $15 \mathrm{~mA}$ per gel. After around 1.5 $\mathrm{h}$ the run was finished and the system was disassembled. The gel was rinsed with ultrapure water, placed into a plastic container filled with staining solution and shaken for $5 \mathrm{~min}$. After destaining overnight, the gel was documented using a transilluminator (Vilber Lourmat, Eberhardzell, Germany). 
Table 3.8: Buffers and solutions used for SDS-PAGE.

\begin{tabular}{|c|c|}
\hline Name & Composition \\
\hline Sample buffer $(2 x)$ & $\begin{array}{l}125 \mathrm{~mm} \text { TRIS/HCl pH } 6.8 \text {, 100 mM DTT, } 20 \%(v / v) \text { glyc- } \\
\text { erol, } 2 \%(w / v) \text { SDS, } 0.02 \%(w / v) \text { bromphenol blue }\end{array}$ \\
\hline Stacking gel & $\begin{array}{lllll}127 \mathrm{~mm} \text { TRIS } / \mathrm{HCl} & \mathrm{pH} 8.8, \quad 5.2 \% & (w / v) & \text { acry- } \\
\text { lamide/bisacrylamide } & (37.5: 1) & \text { stock } & \text { solution, } & 0.1 \% \\
(w / v) \text { SDS } & & & & \end{array}$ \\
\hline Resolving gel & $\begin{array}{lllll}258 \mathrm{~mm} \text { TRIS } / \mathrm{HCl} & \mathrm{pH} 6.8, & 12.5 \%(w / v) & \text { acry- } \\
\text { lamide/bisacrylamide } & (37 \cdot 5: 1) & \text { stock solution, } & 0.1 \% \\
(w / v) \text { SDS } & & & & \end{array}$ \\
\hline $\begin{array}{l}\text { ammonium peroxy- } \\
\text { disulfate (APS) }\end{array}$ & $10 \%(w / v)$ APS in ultrapure water \\
\hline Electrophoresis buffer & $\begin{array}{l}25 \mathrm{~mm} \text { TRIS/HCl, } 192 \mathrm{~mm} \text { glycine, } 0.1 \%(w / v) \text { SDS, } \\
\mathrm{pH} 8.3\end{array}$ \\
\hline $\begin{array}{l}\text { Coomassie blue stain- } \\
\text { ing solution }\end{array}$ & $\begin{array}{l}0.02 \%(w / v) \text { Coomassie Brilliant Blue G250, } 45 \%(v / v) \\
\text { methanol, } 18.5 \%(v / v) \text { glacial acetic acid }\end{array}$ \\
\hline Destaining solution & $5 \%(v / v)$ methanol, $7.5 \%(v / v)$ glacial acetic acid \\
\hline $\begin{array}{l}\text { Low molecular weight } \\
\text { markers }\end{array}$ & $\begin{array}{l}97 \mathrm{kDa} \text { phosphorylase } \mathrm{b}, 66 \mathrm{kDa} \text { albumin, } 45 \mathrm{kDa} \text { oval- } \\
\text { bumin , } 30 \mathrm{kDa} \text { carbonic anhydrase, } 20.1 \mathrm{kDa} \text { trypsin in- } \\
\text { hibitor, } 14.4 \mathrm{kDa} \alpha \text {-lactalalbumin }\end{array}$ \\
\hline
\end{tabular}

\subsubsection{Dialysis}

After SDS-PAGE analysis (section 3.1.5.1), the fractions containing protein were dialyzed against the desired buffer. Dialysis tubing (Carl Roth, Karlsruhe) with a molecular weight cut-off of $14 \mathrm{kDa}$ was cleaned before use according to the procedure provided by the manufacturer. Then, pieces of $10 \mathrm{~cm}$ were soaked in ultrapure water and dialysis buffer, filled with $2 \mathrm{ml}$ to $4 \mathrm{ml}$ of protein solution and sealed with plastic clamps. The tubing was immersed in a large beaker filled with pre-cooled dialysis buffer. The volume of dialysis buffer was at least 200-fold greater than the sample volume. The dialysis was performed stirring overnight at $4{ }^{\circ} \mathrm{C}$. In total, the dialysis buffer was changed three times. Buffers used as dialysis buffer are listed in table 3.9. After dialysis, the concentration of protein was determined as described in section 3.1.5.3. Protein solutions were stored at $4{ }^{\circ} \mathrm{C}$ until use. 
Table 3.9: Protein buffers.

\begin{tabular}{|c|c|}
\hline Name & Composition \\
\hline Protein buffer E1 & $\begin{array}{l}20 \mathrm{~mm} \text { TRIS/HCl, } 50 \mathrm{~mm} \mathrm{KCl,} \mathrm{o.1} \mathrm{mM} \mathrm{EDTA,} \mathrm{o.1} \mathrm{mM} \\
\mathrm{NaN}_{3}, \mathrm{pH} 7.4\end{array}$ \\
\hline Gel filtration buffer & $\begin{array}{l}20 \mathrm{~mm} \text { TRIS/HCl, } 150 \mathrm{~mm} \mathrm{KCl} \text {, o.1 mm EDTA, o.1 mm } \\
\mathrm{NaN}_{3}, \mathrm{pH} 7.4\end{array}$ \\
\hline
\end{tabular}

\subsubsection{Concentration determination}

The concentration of protein was determined via UV absorbance at $\lambda=280 \mathrm{~nm}$. This method is fast as well as convenient since no additional reagents are necessary and protein is not consumed. However, it is only applicable on proteins with aromatic amino acids. The absorbance $A_{280}$ of such proteins is caused by tryptophane and tyrosine, and to a small extent by phenylalanine. ${ }^{[20]}$ According to Beer-Lambert law (equation 3.1), the logarithm of the ratio between the intensity of light before $\left(I_{0}\right)$ and after $(I)$ passage through the protein solution is linearly dependent on the pathlength $l$ and concentration $c$ :

$$
A=-\lg \frac{I}{I_{0}}=\varepsilon l c,
$$

where $\varepsilon$ denotes the molar extinction coefficient specific for the protein. In case of ezrin, the molar extinction coefficient was determined to be $\varepsilon_{280}=66900 \mathrm{M}^{-1} \mathrm{~cm}^{-1}$ using the ProtParam tool. ${ }^{[21]}$ Since nucleic acids show an absorption maximum at $\lambda=260 \mathrm{~nm}$, the absorbance ratio $A_{260} / A_{280}$ is used to assess the nucleic acid contamination of a protein solution. A ratio $A_{260} / A_{280}$ of $\sim 0.5$ ensures that the protein solution is free of such contamination.

\subsection{SURFACE FUNCTIONALIZATION}

Surface functionalization strategies based on the spontaneous assembly of surface active agents (surfactants) offer a rich toolbox for the investigation of biological processes using techniques such as atomic force microscopy (AFM), surface plasmon resonance (SPR), and fluorescence microscopy. Spontaneous assembly processes at surfaces include on the one hand the fabrication of self-assembled monolayers composed of molecules such as alkanethiols and trichlorosilanes and on the other hand the formation of a lipid bilayer. 


\subsubsection{Self-assembled monolayer formation}

The formation of a self-assembled monolayer (SAM) is driven by specific interactions between the surfactant head group and the substrate surface. The degree of order in a monolayer is dependent on factors such as head group affinity of the surfactant to the surface and steric as well as electrostatic effects within the layer. Extensively studied molecules that form well ordered and stable SAMs are for instance long chain alkyl thiols and trichlorosilanes. In 1983, Nuzzo and Allara published the preparation of SAMs by the adsorption of di-n-alkyl disulfides on gold, whereas Maoz and Sagiv showed one year later that alkylsiloxane monolayers are obtained by covalent adsorption of trichlorosilanes onto hydrophilized silicon dioxide. ${ }^{[22,23]}$ SAMs have emerged being an inestimable tool for analyzing interfacial reactions since a wide range of functional groups can be incorporated in the monolayer as a tail group. This feature allows to control surface properties on a molecular level. Depending on the nature of the surfactant, it is for instance possible to render the surface hydrophobic or hydrophilic. Such surface functionalization strategies can be employed to produce either a lipid monolayer on a hydrophobic substrate, e.g. a hybrid membrane, or a physisorbed lipid bilayer on a hydrophilic substrate. ${ }^{[24-26]}$

Beside serving as substrate for hybrid membranes, SAMs are widely used as model systems for the study of surface effects such as adhesion, protein adsorption, and molecular recognition. In particular, the co-adsorption of different surfactants resulting in mixed SAMs is applied to implement specific receptors within the monolayer such as biotin or nitrilotriacetic acid (NTA). ${ }^{[27,28]}$ In addition, selfassembled monolayers are not restricted to planar surfaces but do form as well on curved surfaces such as colloids. ${ }^{[23]}$

\subsubsection{Thiols}

All thiols used in this work were purchased from Prochimia (Sopot, Poland). Before self-assembly, gold coated substrates were cleaned for $2 \mathrm{~min}$ and cantilevers for $30 \mathrm{~s}$ in argon plasma. The substrates were immersed in $2 \mathrm{~mm}$ ethanolic thiol solution. In case of (11-mercaptoundecyl)trimethylammonium $\left(\mathrm{AUT}^{+}\right)$, substrates were incubated overnight at room temperature, whereas cantilevers were placed into a 1:5 mixture of $N$-[5-[[[(20-mercapto-3,6,9-trioxaeicos-1-yl)oxo]carbonyl]amino]-1carboxypentyl]iminodiacetic acid (NTA-thiol) and (1-mercaptoundec-11-yI)tri(ethylene glycol) (matrix thiol) for $1 \mathrm{~h}$. The final thiol concentration was $2 \mathrm{~mm}$. After incubation in thiol solution, the substrates and the cantilevers, respectively, were carefully rinsed with ethanol p.a. and ultrapure water. 


\subsubsection{Silanes}

A silicon substrate $\left(100 \mathrm{~nm} \mathrm{SiO}_{2}\right.$ layer, $\left.0.8 \mathrm{~cm} \times 2.0 \mathrm{~cm}\right)$ was rinsed thoroughly with isopropanol and water. An aqueous solution of $\mathrm{NH}_{3}$ and $\mathrm{H}_{2} \mathrm{O}_{2}\left(\mathrm{H}_{2} \mathrm{O} / \mathrm{NH}_{3} / \mathrm{H}_{2} \mathrm{O}_{2}\right.$ 5:1:1) was used to turn the silicon substrate's surface hydrophilic and remove organic contamination $\left(20 \mathrm{~min}\right.$ at $\left.7 \mathrm{O}^{\circ} \mathrm{C}\right)$. Additional hydrophilization was achieved by oxygen plasma treatment for $2 \mathrm{~min}$. The substrate was immersed in dry toluene containing dodecyl-trichlorosilane (DTS, $2 \%(v / v)$ ) under vacuum for $15 \mathrm{~min}$, thoroughly rinsed with dry toluene, and left overnight under vacuum at $65^{\circ} \mathrm{C}{ }^{[29]} \mathrm{DTS}$ was obtained from Sigma Aldrich (Steinheim, Germany). Toluene p.a. (VWR, Darmstadt, Germany) was dried over 4 A molecular sieves (Carl Roth, Karlsruhe, Germany).

\subsubsection{Solid-supported membrane formation}

In the 1980s, McConnell and co-workers started depositing phospholipids directly onto solid support. Suitable substrates for the formation of solid-supported membranes are for instance silicon dioxide, borosilicate glass, and mica. ${ }^{[24,30-32]}$

\subsubsection{Preparation of lipid films}

The lipids L- $\alpha$-phosphatidylinositol-4,5-bisphosphate $\left(\mathrm{PIP}_{2}\right)$, 1-palmitoyl-2-oleoylsn-glycero-3-phosphocholine (POPC), 1,2-dioleoyl-sn-glycero-3-[( $N$-(5-amino-1-carboxypentyl) iminodiacetic acid)succinyl] (nickel salt) (DOGS-NTA-Ni), and 1,2-dipalmitoyl-sn-glycero-3-phosphocholine (DPPC) were purchased from Avanti polar lipids (Alabaster, USA). The fluorescently labeled PIP 2 analogue Bodipy TMR-PIP 2 (C16) was obtained from Echelon Biosciences (Salt Lake City, USA). Stock solutions of the respective lipids were prepared in chloroform at concentrations ranging from $0.5 \mathrm{mg} / \mathrm{ml}$ to $10 \mathrm{mg} / \mathrm{ml}$ except for $\mathrm{PIP}_{2}$, which was dissolved in a mixture of chloroform/methanol/water (20:9:1) at $1 \mathrm{mg} / \mathrm{ml}$. The particular lipid stock solutions were mixed in a test tube preloaded with $200 \mu \mathrm{l}$ chloroform at the desired molar ratio (total amount of lipid: $0.4 \mathrm{mg}$ to $0.5 \mathrm{mg}$ ). Fluorophores were added in small amounts ( $0.5 \mathrm{~mol} \%$ to $1 \mathrm{~mol} \%)$ in case of fluorescence experiments as indicated in section 4.6. The organic solvent was evaporated with a gentle stream of nitrogen at a temperature above the lipid gel-fluid phase transition. To remove residual solvent, the lipid film was further dried under vacuum for $3 \mathrm{~h}$ at elevated temperature as before. Lipid films were stored at $4{ }^{\circ} \mathrm{C}$ until use. 


\subsubsection{Preparation of small unilamellar vesicles}

Small unilamellar vesicles (SUVs) were prepared by sonication. A lipid film (paragraph 3.2.2.1) was rehydrated by adding $0.5 \mathrm{ml}$ to $1 \mathrm{ml}$ of appropriate buffer, incubated for $20 \mathrm{~min}$ and vortexed for $3 \times 30$ s periods with $5 \mathrm{~min}$ rest in between. The suspension of multilamellar vesicles (MLVs) was transferred to a new Eppendorf cup and sonicated for $2 \times 15 \mathrm{~min}$ using a ultrasonic homogenizer (Sonopuls HD 2070, resonator cup) from Bandelin (Berlin, Germany).

\subsubsection{Preparation of solid-supported membranes}

For atomic force microscopy experiments (section 5.6), fluorescence microscopy and recovery after photobleaching studies (section 4.6) either solid-supported hybrid membranes (SHMs) or physisorbed solid-supported lipid bilayers (SLBs) were prepared. To form SHMs, a substrate was rendered hydrophobic according to section 3.2.1.2 and placed in a measuring chamber. Freshly prepared SUV suspension (section 3.2.2.2) was added and incubated for $2 \mathrm{~h}$ at a temperature above the lipid gel-fluid phase transition. Subsequent rinsing with buffer removed remaining vesicles from the surface. In case of $\mathrm{SLBs}$, a silicon substrate (100 $\mathrm{nm} \mathrm{SiO}_{2}$ layer, $0.8 \mathrm{~cm} \times 2.0 \mathrm{~cm}$ ) was rinsed thoroughly with isopropanol and water. An aqueous solution of $\mathrm{NH}_{3}$ and $\mathrm{H}_{2} \mathrm{O}_{2}\left(\mathrm{H}_{2} \mathrm{O} / \mathrm{NH}_{3} / \mathrm{H}_{2} \mathrm{O}_{2}\right.$ 5:1:1) was used to yield a hydrophilic surface and remove organic contamination $\left(20 \mathrm{~min}\right.$ at $\left.70^{\circ} \mathrm{C}\right)$. The hydrophilized substrate was treated with SUV suspension as described above.

\section{$3 \cdot 3$ BIOPHYSICAL TECHNIQUES}

\subsubsection{Atomic force microscopy}

The atomic force microscope (AFM) was developed in 1985 by G. Binning, C. Gerber and C. F. Quate based upon the scanning tunneling microscope (STM). ${ }^{[33]}$ In contrast to STM, the AFM allows easily the characterization of nonconductive samples. In particular due to the diversity of applications, the AFM is used for surface analysis in many different fields. The applications of AFM encompass high resolution imaging of various materials such as biomolecules, ${ }^{[34]}$ cells, ${ }^{[35]}$ polymers, ${ }^{[36]}$ metals, ${ }^{[37]}$ and ceramics ${ }^{\left[{ }^{38]}\right.}$ as well as detection of forces between individual molecules and surfaces including specific ligand-receptor interactions ${ }^{[39]}$ and the unfolding of protein domains. ${ }^{[40]}$

BASIC PRINCIPLE AND SETUP In contrast to optical microscopes, the AFM does not rely on the use of lenses to generate an image and the resolution is thus not restricted by Abbe's limit. Instead, a flexible cantilever with a sharp tip is used 
to probe the sample surface. The imaging resolution is strongly dependent on the tip shape, that is, the sharper the tip the better the resolution. However, in case of soft samples, often a less sharp tip is employed to avoid damaging the sample. Besides the cantilever, the piezoelectric scanners are essential to allow precise movement of probe and sample. In case of the MFP-3D from Asylum Research (Santa Barbara, USA) the probe is positioned by a Z-piezo scanner, whereas the sample is moved within the plane using the XY-piezo stage (figure 3.1). The optical lever detection technique is applied to measure the bending of the cantilever as response to the mechanical interaction with the sample. ${ }^{\left[{ }^{11}\right]}$ For this purpose, the beam of a super luminescent diode (figure 3.1, red) is focused to the back of the cantilever (yellow) and reflected by its mostly gold coated surface. The light travels through a recollimation lens to a mirror (both in light blue), which directs the light toward the position sensitive detector, a segmented photodiode (green). The cantilever is mounted at approximately $11^{\circ}$ with respect to the sample plane. Force exerted on the cantilever leads to bending and thus changes the angle of the reflected beam as well as its position on the photodiode. Torsion of the cantilever upon lateral forces shifts the beam position vertically on the photodiode. Based on the principle of an optical lever, the AFM is able to detect subnanometer changes. ${ }^{422,43]}$

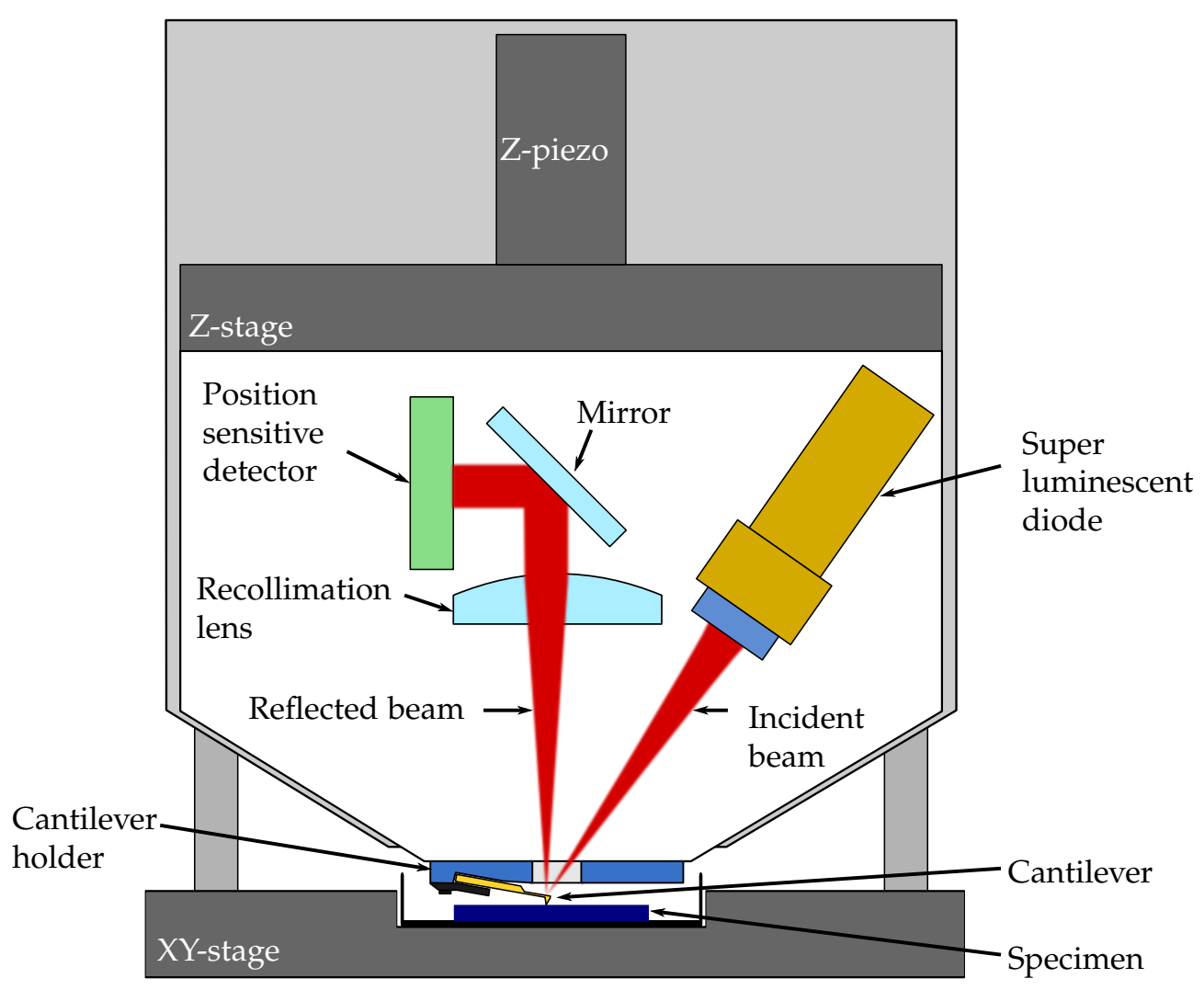

Figure 3.1: Schematic drawing of MFP-3D head. 
TIP-SAMPLE INTERACTION FORCES The AFM technique is characterized by the interaction forces between probe and sample surface. When the tip of a cantilever approaches the sample, diverse intermolecular, surface, and macroscopic interactions with different distance dependencies occur. The most contributing interactions are attractive van der Waals $(\mathrm{VdW})$ forces and repulsive Pauli forces. The tip surface interaction can thus be described to a first approximation by the Lennard-Jones potential (equation 3.2). The interaction potential $V$ as a function of the distance $D$ between a pair of particles is given by:

$$
V(D)=4 \epsilon\left[\frac{\sigma}{D^{12}}-\frac{\sigma}{D^{6}}\right]
$$

where $\epsilon$ denotes the depth of the potential well and $\sigma$ the distance at which the potential between two particles equals zero. The Lennard-Jones potential is composed of the reciprocal sixth-power $\mathrm{VdW}$ term, which is attractive, as well as the reciprocal 12th-power repulsive term, describing the Pauli repulsion due to overlapping electron orbitals. The derivative of the Lennard-Jones potential is depicted in figure 3.2, showing two different regimes. On the one hand, the long range $\mathrm{VdW}$ forces lead to attraction until $F=0$ corresponding to the energy minimum. On the other hand, any further approach results in increasing repulsive forces $F>0$. Depending on the regime, different AFM imaging modes are possible. The non-contact mode (figure 3.2, NC) is operated in the attractive regime, however close to the surface. In case of intermittent contact mode (figure 3.2, IC), the tip touches the sample periodically, transitioning from repulsive to attractive regime and vice versa. Contact mode (figure 3.2, C) is operated fully in the repulsive regime, where the cantilever tip is in constant contact with the surface (section 3.3.1.2).

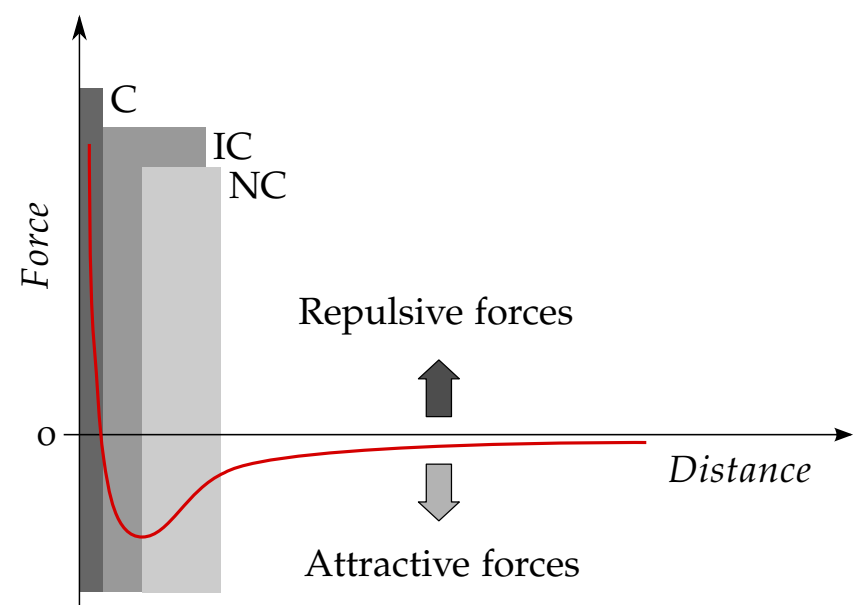

Figure 3.2: The interaction force is described by the derivative of the Lennard-Jones potential with respect to distance $D$. Different AFM imaging modes are depicted as a function of tip-sample distance (C: contact mode, IC: intermittent contact mode, NC: non-contact mode). 
The tip sample interaction is not described adequately by the interaction between two particles, though. This can be illustrated by deriving expressions for the VdW contribution of both, particle-particle and macroscopic body interaction. VdW forces in general can be divided into three categories. The Keesom force is characterized by the interaction between permanent dipoles, whereas the Debye force is given by the interaction between a permanent dipole and an induced dipole. These forces act only between polar molecules and thus are referred to as polar VdW contribution. The most important VdW contribution, the dispersion contribution, is the London force acting between induced dipoles, which is abundant between all molecules, hence. The overall VdW contribution $w$ to the interaction potential between two small particles is given by: ${ }^{[44]}$

$$
w(D)=-\frac{C_{\mathrm{K}}+C_{\mathrm{L}}+C_{\mathrm{D}}}{D^{6}}=-\frac{C_{\mathrm{VdW}}}{D^{6}},
$$

where $C_{\mathrm{K}}, C_{\mathrm{L}}$ and $C_{\mathrm{D}}$ denote contributions of Keesom, London, and Debye interactions, respectively. The force $F$ is the derivative with respect to $D$ :

$$
F(D)=\frac{d w(D)}{d D}=-\frac{6 C_{\mathrm{VdW}}}{D^{7}} .
$$

Equation 3.4, however, describes the interaction between two single particles, a situation that is not likely in AFM experiments. When the tip comes in contact with the surface, it is an interaction between macroscopic bodies rather than atoms or small molecules. Therefore, it is necessary to scale up the VdW forces. This has been done by Hamaker based on the pairwise addition of interactions with regard to several different geometries, such as two surfaces, two spheres, atom-surface, and sphere-surface. The interaction energy of two planar surfaces at a distance $D$ can be considered in terms of energy per unit surface area: ${ }^{[45]}$

$$
W(D)=-\frac{\pi C \rho^{2}}{6} \int_{\mathrm{D}}^{\infty} \frac{d r}{r^{3}}=-\frac{A_{\mathrm{H}}}{12 \pi D^{2}} \quad \text { with } \quad A_{\mathrm{H}}=\pi^{2} C \rho^{2}
$$

where $A_{\mathrm{H}}$ denotes the Hamaker constant with $C$ being the coefficient in the surfacesurface potential and $\rho$ the number of atoms per unit volume. In case of two interacting bodies that are of complex shape, integration as described above will lead to long and complicated expressions. Derjaguin, however, overcame these issues by deriving a relationship between the energy per surface area of two planar surfaces and the energy between two arbitrarily shaped bodies. His assumptions are valid as long as the distance between the interacting bodies and the range of the interaction is small compared to the radii of curvature. According to the 
Derjaguin's approximation, the force law $F(D)$ between two spheres is related to the interaction free energy per surface area $W(D)$ between two planar surfaces: ${ }^{[46]}$

$$
F(D)=2 \pi\left(\frac{R_{1} R_{2}}{R_{1}+R_{2}}\right) W(D)
$$

where $R_{1}$ and $R_{2}$ denote the sphere radii. The tip sample interaction can be approximated as a sphere in contact with a flat surface. This geometry is a special case of the interaction between two spheres with one sphere being much larger. If we consider $R_{1} \ll R_{2}$, equation 3.6 then becomes:

$$
F(D)=2 \pi R_{1} W(D)
$$

Combining equation 3.5 and equation 3.7 gives

$$
F(D)=-\frac{A_{\mathrm{H}} R_{1}}{6 D^{2}}
$$

This interaction law depends on the distance $D$, the sphere radius $R_{1}$, and the Hamaker constant $A_{\mathrm{H}}$. It should be pointed out that the interaction between macroscopic bodies, being proportional to the inverse square of distance (equation 3.5), is of long range nature compared to the one between small particles, having an inverse sixth-power distance dependence (equation 3.3). Therefore, the distance dependence of the force in an AFM experiment differs from figure 3.2 as such that the attractive regime is broadened toward larger distances.

SPRING CONSTANT DETERMINATION The calibration of a cantilever to assess its spring constant is pivotal to force spectroscopic measurements in particular. In principle, it is possible to calculate the spring constant of a cantilever from its geometry and material properties. For a rectangular cantilever, the spring constant $k_{c}$ is given by: ${ }^{[47]}$

$$
k_{c}=\frac{F}{Z_{c}}=\frac{E w_{\mathrm{c}} t_{\mathrm{c}}^{3}}{4 L^{3}}
$$

where $w_{\mathrm{c}}$ denotes the width, $t_{\mathrm{c}}$ the thickness, and $L$ the length of the cantilever. The Young's modulus of the material is given by E. According to equation 3.9 a cantilever should be long and thin to show large deflections at small forces, that is, a high sensitivity. However, a high resonance frequency is desirable to ensure both, reduced interferences with external vibrations and high time resolution. Thus, a compromise is achieved by using thin and short cantilevers, which have high resonance frequency and high sensitivity. ${ }^{[44]}$ The calculation of spring constants from equation 3.9 yields different values as compared to experimentally assessed ones, though. This is due to the fact that the Young's modulus of silicon ni- 
tride cantilevers is strongly dependent on manufacturing conditions. Furthermore, the determination of thickness, length and width are prone to errors as well. ${ }^{[48]}$ In addition most cantilevers are coated with gold on the back to enhance the reflectivity which makes it even harder to determine elastic properties. ${ }^{[49]}$ Due to these uncertainties many different techniques have been contrived over the past two decades such as the reference spring method ${ }^{\left[5^{\circ}\right]}$ and the nondestructive added mass method ${ }^{[51]}$ to improve the determination of spring constants. However, Hutter and Bechhofer devised a method, which does not rely on attachment of any mass or reference cantilevers. This technique is implemented in many commercial AFMs and is based on measuring the intensity of thermal noise. If the cantilever is modeled as a harmonic oscillator and the equipartition theorem is applied to the first flexural mode in the cantilever, the mean square cantilever displacement $\left\langle\Delta Z_{c}^{2}\right\rangle$ is related as follows: ${ }^{[49]}$

$$
\frac{1}{2} k_{\mathrm{c}}\left\langle\Delta Z_{\mathrm{c}}^{2}\right\rangle=\frac{1}{2} k_{\mathrm{B}} T
$$

where $k_{\mathrm{c}}$ denotes the spring constant of the cantilever, $k_{\mathrm{B}}$ the Boltzmann's constant, and $T$ the temperature. The spring constant of the cantilever is readily obtained when equation 3.10 is solved for $k_{\mathrm{c}}$ :

$$
k_{c}=\frac{k_{\mathrm{B}} T}{\left\langle\Delta Z_{\mathrm{c}}^{2}\right\rangle} .
$$

The mean square cantilever displacement $\left\langle\Delta Z_{\mathrm{c}}^{2}\right\rangle$ is obtained from the power spectral density. First, the thermal fluctuations of the cantilever are recorded as the amplitude of the cantilever response in units of $\mathrm{V} / \sqrt{\mathrm{Hz}}$ and second, the inverse optical lever sensitivity ( $\operatorname{nv} O L S$ ) is used to convert the thermal vibrational amplitude into units of $\mathrm{m} / \sqrt{\mathrm{Hz}}$. This conversion factor is obtained by recording a deflection displacement curve on a hard surface, while measuring the voltage response $\Delta V$ of the photodiode:

$$
\operatorname{InvOLS}=\frac{\Delta \mathrm{Z}_{\mathrm{p}}}{\Delta V}
$$

where $\Delta Z_{\mathrm{p}}$ is the change in Z-piezo height. However, these measurements are not as straightforward as described above. On the one hand, contributions from higher flexural modes are neglected since the equipartition theorem only refers to single modes. On the other hand, the inclination rather than the deflection of the cantilever is measured by the optical lever detection. Thus, two major corrections have been implemented over the years. One is that a mode correction factor $\beta$ has been introduced to take into account that the thermal noise measurement is based on a single mode of a real cantilever rather than a simple harmonic oscillator. 
The second correction considers that the shape of a cantilever changes whether the cantilever vibrates freely by thermal fluctuations or it is end-loaded. Such deviations are taken into account by introducing the correction factor $\chi:^{\left[5^{2-54]}\right.}$

$$
k_{\mathrm{c}}=\frac{\beta k_{\mathrm{B}} T}{\chi^{2}\left\langle\Delta Z_{\mathrm{c}}^{2}\right\rangle} \quad \text { with } \quad \beta=0.971 \quad \text { and } \quad \chi=1.09 \text {. }
$$

The assumption of $\chi=1.09$ however is only valid in the case of a small optical spot at the very end of a large cantilever. Deviations in $\chi$ affect the determination of the spring constant to a great extent since $\left\langle\Delta Z_{\mathrm{c}}^{2}\right\rangle$ and $k_{\mathrm{c}}$ are quadratically related. ${ }^{\text {[54] }}$ The equations 3.10 to 3.13 are limited to rectangular cantilevers. One additional consideration is necessary since cantilevers are usually mounted in a slightly tilted way to ensure that only the tip comes into contact with the sample surface. In case of the MFP-3D, the cantilever tilt $\theta$ amounts to $11^{\circ}$. Division of the measured spring constant by the term $\cos ^{2} \theta=0.964$ diminishes the contribution of the tilted cantilever. ${ }^{[55,56]}$

\subsubsection{Force measurements}

Besides the application of the AFM as high resolution imaging tool, the use as highly sensitive force measuring device has been in the focus of increased interest over the past 20 years. In a force measurement, force distance curves are recorded to give information about the interaction between tip and sample as a function of tip sample separation. This is done by the Z-piezo scanner as response to a command voltage, ramping the AFM head up and down along the z-axis. Figure $3 \cdot 3$ shows a typical course of a deflection displacement curve comprising approach (red) and retraction curve (blue). Initially, no force is exerted on the cantilever since it is far away from the surface (A). As soon as the cantilever comes into proximity of the surface, it may be affected by long range surface forces such as electrostatics $(\sim 1 \mu \mathrm{m})$. In the range of $10 \mathrm{~nm}$ to $100 \mathrm{~nm}$, the cantilever experiences $\mathrm{VdW}$ attraction. ${ }^{[46]}$ These long range $\mathrm{VdW}$ forces originate in the interaction between macroscopic bodies, that is between cantilever tip and sample surface (section 3.3.1). When the gradient of attractive surface forces overcomes the restoring force of the cantilever, the equilibrium between spring and surface forces is lost and as a result, the cantilever jumps into contact with the surface (B). This mechanical instability is also referred to as "jump-in" or "snap-on". Further movement of the Z-piezo towards the surface leads to increasing deflection of the cantilever (C). Depending on the sample, deformation may occur. If the cantilever is retracted subsequently, the deflection decreases. The tip stays in contact with the surface until the restoring force of the cantilever exceeds the adhesion force $F_{a d}$. Adhesion causes the cantilever to bend down and consequently, to jump off the surface at a 
distance further away from the surface as in case of the initial jump-in (D). After the jump-off, the cantilever returns to its baseline deflection (E).
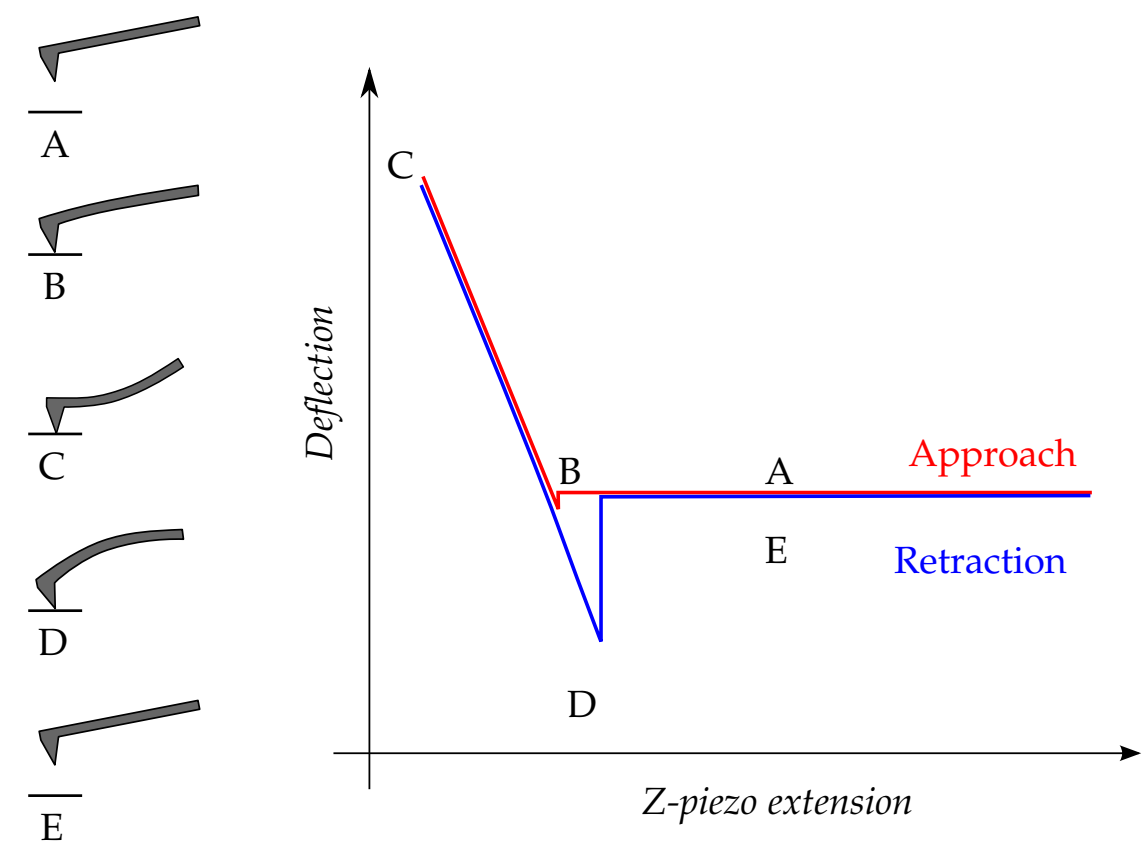

Figure 3.3: Drawing of cantilever approaching and retracting from surface and its deflection as a response to surface contact. During the approach phase $\mathbf{A}$, no tip sample interaction is detected. At point $\mathbf{B}$, the cantilever jumps into contact with the sample surface due to attractive surface forces. Further approach leads to increasing deflection of the cantilever until a preset deflection value is reached (C). During retraction, the tip stays in contact with the surface due to adhesive forces. Once they are overcome by the cantilever's restoring force (D), the cantilever returns to its baseline deflection (E).

CONVERSION OF FORCE CURVES To obtain a force distance curve, the raw data acquired from such a measurement, that is, cantilever deflection as a function of Z-piezo displacement, requires two corrections. First, the measured deflection is converted into a force $F$ according to Hooke's law:

$$
F=-k_{\mathrm{c}} Z_{\mathrm{c}}
$$

For this purpose, the spring constant of the cantilever $k_{c}$, determined as described in paragraph 3.3.1, is multiplied by the cantilever deflection $Z_{c}$ in units of $\mathrm{nm}$. The InvOLS value obtained in the course of spring constant determination is used for this conversion. Second, the Z-piezo displacement $Z_{p}$ has to be transformed into the actual tip sample separation. This correction is necessary as soon as the cantilever tip comes into contact with the surface. Consequently, the cantilever 
deflection $Z_{\mathrm{c}}$ as well as the sample indentation $\delta$ has to be considered. Subtracting these contributions from $Z_{\mathrm{p}}$ yields the tip sample separation $D$ :

$$
D=Z_{\mathrm{p}}-\left(Z_{\mathrm{c}}+\delta\right)
$$

However, the indentation of many inorganic solids including silicon wafers and glass by a soft cantilever is neglectable since $\delta \ll \Delta Z_{\text {c }}$. Correcting the tip sample separation changes the force displacement curve in such a way that the tip sample separation in the contact regime remains zero, while the force is increased by the pushing cantilever.

\subsubsection{Imaging}

The AFM has emerged as a divers surface characterization technique in many different fields ranging from material sciences to life science. Imaging of surfaces at atomic resolution is possible under ultra high vacuum, while imaging in fluid allows the characterization of biological specimen under physiological conditions. Different AFM imaging modes have been developed to meet the requirements of various applications.

CONTACT MODE In contact mode, the cantilever raster scans the sample while the tip is in permanent mechanical contact with the surface (figure 3.2). Two modes of operation are applicable: constant height mode and constant force mode. In case of constant height mode, the height of the Z-piezo scanner relative to the sample is fixed while the cantilever deflection is recorded. From the variations in deflection, topographic information is obtained. However, this mode of operation can only be applied in case of very flat surfaces otherwise the sample would be damaged. In case of constant force mode, the deflection of the cantilever is the input signal to a feedback loop, which adjusts the height of the Z-piezo scanner as a response to the sample's topography by keeping the deflection constant. The image is generated from the variations of the Z-piezo scanner. This mode is generally preferred compared to the constant height mode since rough samples can be characterized without major damage. One advantage of the contact mode is that it allows fast scanning. Imaging soft samples without damaging them, however, is often difficult due to frictional forces. ${ }^{[57]}$

INTERMITTENT CONTACT MODE The intermittent contact mode or referred to as tapping mode is a resonant mode. The cantilever is excited by a piezoelectric actuator and oscillates at a frequency slightly below its resonance frequency close to the surface. The tip periodically touches the surface and experiences attractive and repulsive forces, respectively (figure 3.2). As a result of these interactions 
and because the cantilever is driven at a fixed frequency, the vibration amplitude decreases upon contact with the surface. While scanning in intermittent contact mode, the tip-sample separation is adjusted by a feedback loop, thus maintaining the amplitude constant. This information is used to generate an image of the topographic features. In addition to the vibration amplitude, the phase lag of the cantilever response relative to the actuator is recorded. This phase lag gives information about differences in material properties such as changes in adhesion or elasticity. An additional advantage over the contact mode is, that the tip is only in intermittent contact with the sample and thus lateral forces are significantly reduced. However, the scanning speed is much lower in case of intermittent mode compared to contact mode. ${ }^{[58]}$

EXPERIMENTAL Details In this work, single molecule force spectroscopy as well as colloidal probe microscopy were employed to investigate the interaction between filamentous actin and ezrin depending on its activation state. An extended description of experimental details can be found in section 6.6. Ezrin wildtype, ezrin $\mathrm{T}_{567} \mathrm{~A}$ and $\mathrm{T}_{56} \mathrm{D}$ were imaged on solid-supported DPPC membranes containing either $\mathrm{PIP}_{2}$ or DOGS-NTA-Ni as receptor lipid. A detailed description of the experimental procedure can be found in section 5.6.

\subsubsection{Confocal laser scanning microscopy}

The confocal laser scanning microscopy (CLSM) has evolved as a fundamental noninvasive imaging tool in many research fields including material science and life science. Its success is based on both the improved resolution compared with conventional wide-field fluorescence microscopy and the ability of optical sectioning, which allows reconstruction of three-dimensional features. In a conventional fluorescence microscope, a considerable portion of the sample is illuminated, thus resulting in fluorescence emission not only from the focal plane, but also from above and below. This out-of-focus light blurs the image and hence impairs resolution and contrast. In CLSM, however, most of the out-of focus light is excluded by the use of a spatial filter, commonly named as confocal aperture or pinhole. The term confocal refers to the fact that this filter is situated in a conjugated focal plane. Further decrease of out-of-focus fluorescence is achieved by pointwise illumination of the sample and consequently sequential signal detection. Topographic information of a sample can be reconstructed by recording a series of confocal two-dimensional images as a stack in z-direction. The $\mathrm{x}, \mathrm{y}$-resolution of a CLSM under typical fluorescence conditions is approximately $0.2 \mu \mathrm{m}$, whereas the resolution in z-direction is in the range of $0.7 \mu \mathrm{m} .{ }^{[59,60]}$ 


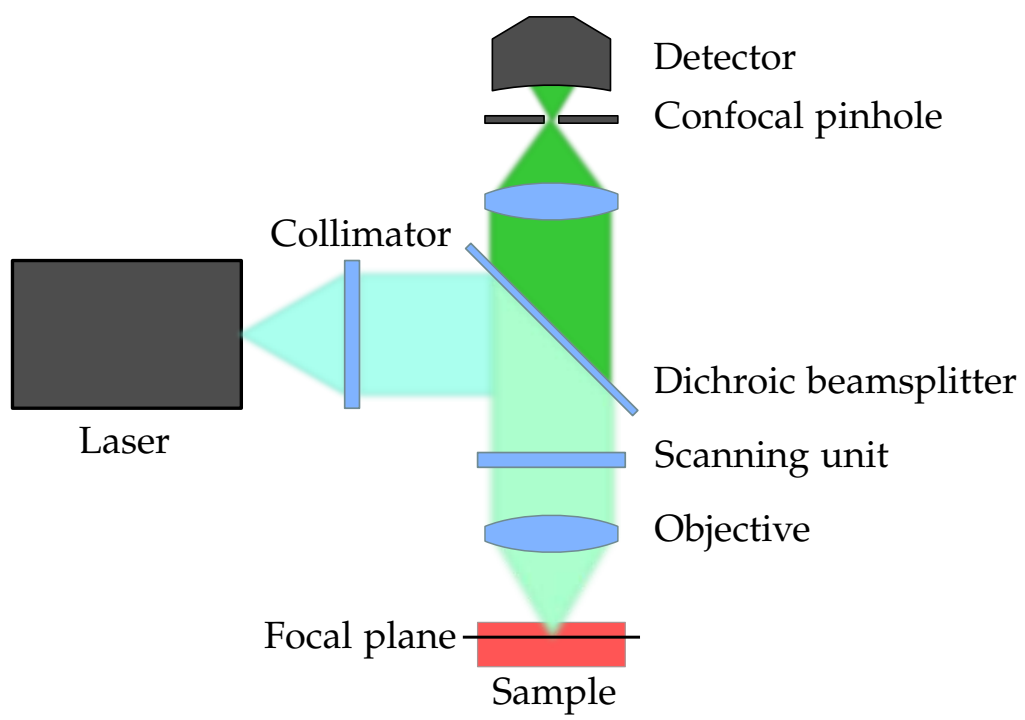

Figure 3.4: Schematic setup of a confocal laser scanning microscope.

The confocal approach is depicted in figure 3.4. The incident laser beam is parallelized by the collimator lens and reflected by the dichroic beamsplitter into the light path of the microscope. The objective focuses the beam to a diffraction limited spot onto the sample. The longer wavelength fluorescent light emitted from the sample travels through the objective and passes the dichroic beamsplitter. Only fluorescent light originated from the focal plane of the objective passes through the confocal pinhole towards the detector, whereas any out-of-focus light is excluded. A CLSM image is generated sequentially, that is, the laser beam is scanned over the sample in a raster pattern. Therefore, no spatial resolution of the detector is necessary. ${ }^{[59]}$

\subsubsection{Fluorescence recovery after photobleaching}

In 1972, Singer and Nicolson published the fluid mosaic model describing biological membranes as two-dimensional fluids composed of diffusing lipids and proteins. ${ }^{[61]}$ During that time, a widespread interest in the investigation of transport processes within lipid membranes lead, among other things, to the development of fluorescence recovery after photobleaching (FRAP). ${ }^{[62]}$ Fluorescent molecules within a small region are photobleached irreversibly by a short light pulse of high intensity. Immediately after photobleaching, the fluorescence recovery due to diffusional mixing of bleached and unbleached species is monitored using an attenuated light beam. This technique is able to disclose dynamics underlying the steady-state distribution of fluorescent molecules. Fluorescence recovery is either observed or not depending on whether the fluorophores are mobile or immobile. The first approach to quantify diffusion coefficients and immobile fractions was published by 
Axelrod et al. ${ }^{\left[6_{3}\right]}$ Assuming diffusion according to Fick's second law in two dimensions, the diffusion coefficient $D$ of the fluorophor is given by:

$$
D=\frac{w_{\mathrm{G}}^{2}}{4 \tau_{\mathrm{D}}}
$$

where $w_{\mathrm{G}}$ represents the Gauss radius of the circular bleached spot and $\tau_{\mathrm{D}}$ is the characteristic diffusion time. However, this approach relies on the assumptions that the bleaching profile is of Gaussian shape, the photobleaching proceeds as an irreversible first-order reaction, and that the bleach puls is short compared to the characteristic time of recovery. ${ }^{[64]}$ One approach, which does not rely on the knowledge of bleaching profile and geometry, is based on a Hankel transformation of the acquired data. In comparison with traditional FRAP analysis methods, this method offers advantages including compensation for temporal variations in intensity, diminished influence of noise, and shape independence of the bleached area. Therefore, the Hankel transform method is in particular suitable for the analysis of experiments affected by nonideal conditions such as photobleaching during recovery and drifts in illumination. ${ }^{[65]}$

EXPERIMENTAL DetAils Confocal imaging as well as FRAP experiments were performed on the LSM 710 (Carl Zeiss, Jena, Germany). The confocal microscope was equipped with a water immersion $63 x$ objective with a numerical aperture $N A=1$ (W Plan Achromat, Carl Zeiss, Jena, Germany) and operated with the software Zen 2008. An Ar-laser was used for the excitation of AlexaFluor488 phalloidin $\left(\lambda_{\mathrm{ex}}=488 \mathrm{~nm}, \lambda_{\mathrm{em}}=495 \mathrm{~nm}\right.$ to $\left.575 \mathrm{~nm}\right)$, the perylene fluorescence was excited at $\lambda_{\mathrm{ex}}=405 \mathrm{~nm}$ by a diode laser and detected at $\lambda_{\mathrm{em}}=410 \mathrm{~nm}$ to $480 \mathrm{~nm}$, and a HeNelaser was chosen for the excitation of Bodipy-TMR-PI $(4,5) \mathrm{P} 2\left(\lambda_{\mathrm{ex}}=561 \mathrm{~nm}, \lambda_{\mathrm{em}}=\right.$ $574 \mathrm{~nm}$ to $619 \mathrm{~nm})$.

DATA ANALysis The Hankel transform method published by Jönsson et al. was used to analyze the acquired data. ${ }^{[65]}$ The analysis was performed using the program frap_analysis with MATLAB R2012b. ${ }^{[66]}$ First, an image sequence containing at least one pre-bleach frame was loaded. Secondly, the number of post-bleach frames as well as the dark count value was specified. The latter was calculated from a selected area within the bleached region. The largest radial value $r_{\text {max }}$ with respect to the center of the bleached region was defined and data specific values such as pixelwidth (in $\mu \mathrm{m}$ ) and frame time (in s) were entered. In the next step, the bleached region was defined by drawing a polygon around its edges. After setting all parameters, the data fitting window was opened, displaying a plot of the normalized Hankel transform $F(k, t) / F(k, 0)$ as a function of both $k$ and $4 \pi^{2} k^{2} t$. The highest value of $k$ that should be used for the FRAP analysis was determined from 
the plot of $F(k, t) / F(k, 0)$ vs $4 \pi^{2} k^{2} t$ as such that the normalized Hankel transform followed an exponential decay of the lowest order possible. A general equation for the determination of $F(k, t)$ is given, considering a single as well as a two component system, either with or without immobile fraction:

$$
\begin{aligned}
F(k, t)= & F(k, 0)\left[\left(1-\gamma_{2}-\gamma_{0}\right) \exp \left(-4 \pi^{2} k^{2} D_{1} t\right)\right. \\
& \left.+\gamma_{2} \exp \left(-4 \pi^{2} k^{2} D_{2} t\right)+\gamma_{0}\right]
\end{aligned}
$$

where $D_{1}$ and $D_{2}$ are diffusion coefficients of components 1 and $2, \gamma_{0}$ is the fraction of immobile molecules and $\gamma_{2}$ is the fraction of the second component. Assuming that we deal with diffusion of a single component with an immobile fraction of molecules, that is $D_{2}=\gamma_{2}=0$, equation 3.17 is simplified to:

$$
F(k, t)=F(k, 0)\left[\left(1-\gamma_{0}\right) \exp \left(-4 \pi^{2} k^{2} D_{1} t\right)+\gamma_{0}\right]
$$

All data were fitted according to equation 3.18 to obtain the diffusion coefficient $D_{1}$ as well as the immobile fraction $\gamma_{0}$.

\subsubsection{Direct optical sensing techniques}

Direct optical sensing methods have gained more and more attention during the last decades, since labeling of any kind can influence the bioactivity of the target molecule. These methods can be divided into two classes: (1) the reflectometric methods such as reflectometric interference spectroscopy (RIfS) and ellipsometry and (2) evanescent field techniques such as surface plasmon resonance spectroscopy. $[67,68]$

\subsubsection{Reflectometric interference spectroscopy}

Reflectometric interference spectroscopy (RIfS) is based on the reflection of white light at the interfaces of a transparent layer system. Superposition of the partially reflected beams generates an interference pattern, which leads to either destructive or constructive interference, depending on wavelength, angle of incidence and optical thickness of the layer system. The optical thickness $O T$ is the product of refractive index $n$ and physical thickness $d$ of the interference layer:

$$
O T=n d
$$


Assuming normal irradiation leads to the following conditions for interference: Maximum constructive interference, that is maximum intensity, is achieved, if the optical path length is an integer multiple $m$ of the wavelength $\lambda_{\text {max }}$ :

$$
m \lambda_{\max }=2 n d,
$$

whereas destructive interference results in minimum intensity fulfilling the following condition: ${ }^{[69]}$

$$
\left(m+\frac{1}{2}\right) \lambda_{\min }=2 n d
$$

In this work, a silicon wafer with a $5 \mu \mathrm{m}$ layer of silicon dioxide is employed as transducer. Incident light is on the one hand directly reflected from the substrate surface, but on the other hand refracted into the silicon dioxide layer where it travels toward the $\mathrm{Si} / \mathrm{SiO}_{2}$-interface from which it is reflected again (figure 3.5, black arrows). Superposition of refracted and reflected light generates an interference spectrum (figure 3.5, black curve). The refractive indices of silicon dioxide, lipid bilayers and proteins are in a similar range. ${ }^{\left[{ }^{[0-72]}\right.}$ Thus, the thickness of the interference layer is increased upon membrane deposition and protein binding. The greater optical path difference between refracted and reflected light (figure 3.5, gray, dashed arrows) produces a red shifted interference spectrum (figure 3.5, gray, dashed curve).
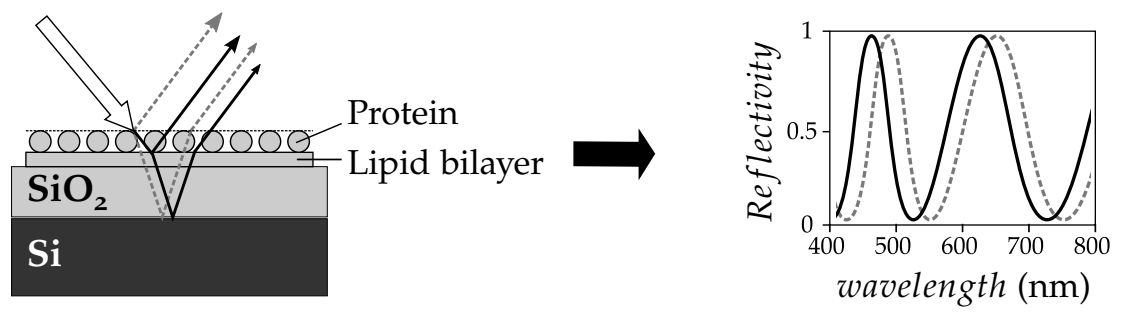

Figure 3.5: Basic principle of RIfS. The initial interference layer is formed by a lipid bilayer and the $\mathrm{SiO}_{2}$-layer of the transducer chip. White light illumination of the initial layer and superposition of the partially reflected beams (black arrows) produces an interference spectrum (black curve). Protein binding (gray arrows, dashed) leads to a thickening of the interference layer resulting in a red shifted interference spectrum (gray curve, dashed).

EXPERIMENTAL SETUP The setup comprises a tungsten halogen lamp, a yshaped optical fiber and an UV-VIS spectrometer all purchased from Ocean Optics (Dunedin, USA). Silicon wafers with a $5 \mu \mathrm{m}$ layer of silicon dioxide were used as transducer chips (ABC - Active business company, Munich, Germany). The light emitted by a tungsten halogen lamp is coupled into an optical fiber and transmitted to a flow-through cell. In this chamber, the silicon chip is fixed by an acrylic glass 
cover containing the flow channel. The transducer is illuminated perpendicularly to its surface, the light is reflected back into the optical fiber and transmitted to a spectrometer. The spectrometer is operated with standard software from OceanOptics.

DATA PROCESSING The recorded intensity spectrum is converted into a reflectivity spectrum according to the following equation: ${ }^{[73]}$

$$
R=\frac{I_{\mathrm{m}}-I_{\text {dark }}}{I_{\text {ref }}-I_{\text {dark }}}
$$

$R$ denotes the reflectivity, $I_{\mathrm{m}}$ the measured intensity spectrum, $I_{\text {ref }}$ the reference intensity spectrum of a purely reflective surface, and $I_{\text {dark }}$ the dark current of the spectrometer. The parameters of the following equation (equation 3.23) are fitted to the reflectivity spectrum (equation 3.22) to obtain the change in optical thickness. The fitting procedure is carried out using the RIfS evaluation tool provided by Milena Stephan.

$$
R(\lambda)=\frac{r_{1}^{2}+2 r_{1} r_{2} \cos \left(\frac{4 \pi}{\lambda} O T\right)+r_{2}^{2}}{1+2 r_{1} r_{2} \cos \left(\frac{4 \pi}{\lambda} O T\right)+r_{1}^{2} r_{2}^{2}}
$$

$r_{1}$ and $r_{2}$ denote the Fresnel coefficients of the respective interfaces. Assuming perpendicular incidence of light, the Fresnel coefficient of the interface is given by:

$$
r=\frac{n_{1}-n_{2}}{n_{1}+n_{2}}
$$

where $n_{1}$ and $n_{2}$ denote the refractive indices of the adjacent layers. The refractive indices at the sodium D-line $(589 \mathrm{~nm})$ of water, silicon dioxide, and silicon amount to $n_{\mathrm{H}_{2} \mathrm{O}}=1.3316, n_{\mathrm{SiO}_{2}}=1.5442$, and $n_{\mathrm{Si}}=3.8714$, respectively. ${ }^{\text {[74] }}$

\subsubsection{Surface plasmon resonance}

Surface plasmon resonance (SPR) is a charge density wave phenomenon that occurs at the interface between a metal and a dielectricum when light irradiated under specific conditions couples with the conduction electrons near the metal surface. However, only metals that are described by the free electron model such as gold and silver are suitable for optical excitation of surface plasmons (SPs). ${ }^{[75]}$ SPs are collective electron oscillations propagating along the metal-dielectricum interface. The amplitude of such a transverse magnetic wave decays exponentially on either 
side. Solving Maxwell's equations using appropriate boundary conditions, the frequency dependent wavevector $\boldsymbol{k}_{\mathrm{sp}}$ of a SP can be described as follows: ${ }^{[76]}$

$$
\boldsymbol{k}_{\mathrm{sp}}=\frac{\omega}{c} \sqrt{\frac{\varepsilon_{\mathrm{d}} \varepsilon_{\mathrm{m}}}{\varepsilon_{\mathrm{d}}+\varepsilon_{\mathrm{m}}}},
$$

where $\omega$ denotes the circular frequency, $c$ the speed of light, and $\varepsilon$ the dielectric constants for metal (subscript $\mathrm{m}$ ) and dielectric medium (subscript $\mathrm{d}$ ). If the propagation of a photon in dielectric medium is considered,

$$
k_{\text {photon }}=\frac{\omega}{c} \sqrt{\varepsilon_{\mathrm{d}}}
$$

it follows that resonant coupling, that is transformation of photons into plasmons,

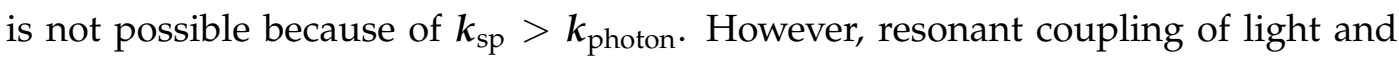
SPs can be achieved as the wavevector of light is increased by a third dielectric medium of high refractive index $\left(\varepsilon>\varepsilon_{\mathrm{d}}\right)$. For this purpose, a prism is commonly used as coupling device. Under attenuated total reflection (ATR) conditions, the $x$-component of the light wavevector $k_{\text {photon, } \mathrm{x}}$ is given by:

$$
k_{\text {photon, } \mathrm{x}}=\frac{\omega}{c} \sqrt{\varepsilon_{\text {prism }}} \sin \Theta_{\mathrm{i}},
$$

where $\varepsilon_{\text {prism }}$ denotes the refractive index of the prism and $\Theta_{\mathrm{i}}$ the angle of incidence above the critical angle $\Theta_{c}$.

One geometry applying a prism coupler to meet ATR criteria is the Kretschmann configuration (figure 3.6). A prism with high refractive index is in contact with a thin metal layer. P-polarized light, passing from the optically denser prism to the less dense metal at an angle of incidence $\Theta_{i}$ above a critical angle $\Theta_{c}$, is totally reflected from the interface. Despite total internal reflection, a component of this light, the evanescent wave, penetrates the interface, exponentially decaying into the metal layer. If the metal layer is sufficiently thin (below $100 \mathrm{~nm}$ for visible and nearinfrared light), the evanescent wave is able to pass through the metal and to couple with SPs on the other side. When the coupling condition $\boldsymbol{k}_{\mathrm{x}}=\boldsymbol{k}_{\mathrm{sp}}$ is matched, a characteristic drop in intensity of the reflected light is observed (SPR dip) due to the energy transfer between the evanescent wave and SPs. Changes in refractive index within the evanescent wave's penetration range alters the wavevector and thus results in a different plasmon resonance angle $\Theta_{i}$ (figure 3.7 A). This angle shift correlates with the adsorption and desorption of molecules, respectively. The kinetics of an adsorption/desorption process is obtained by monitoring the intensity of reflected light as a function of time (figure $3 \cdot 7$ B). ${ }^{[77,78]}$ 


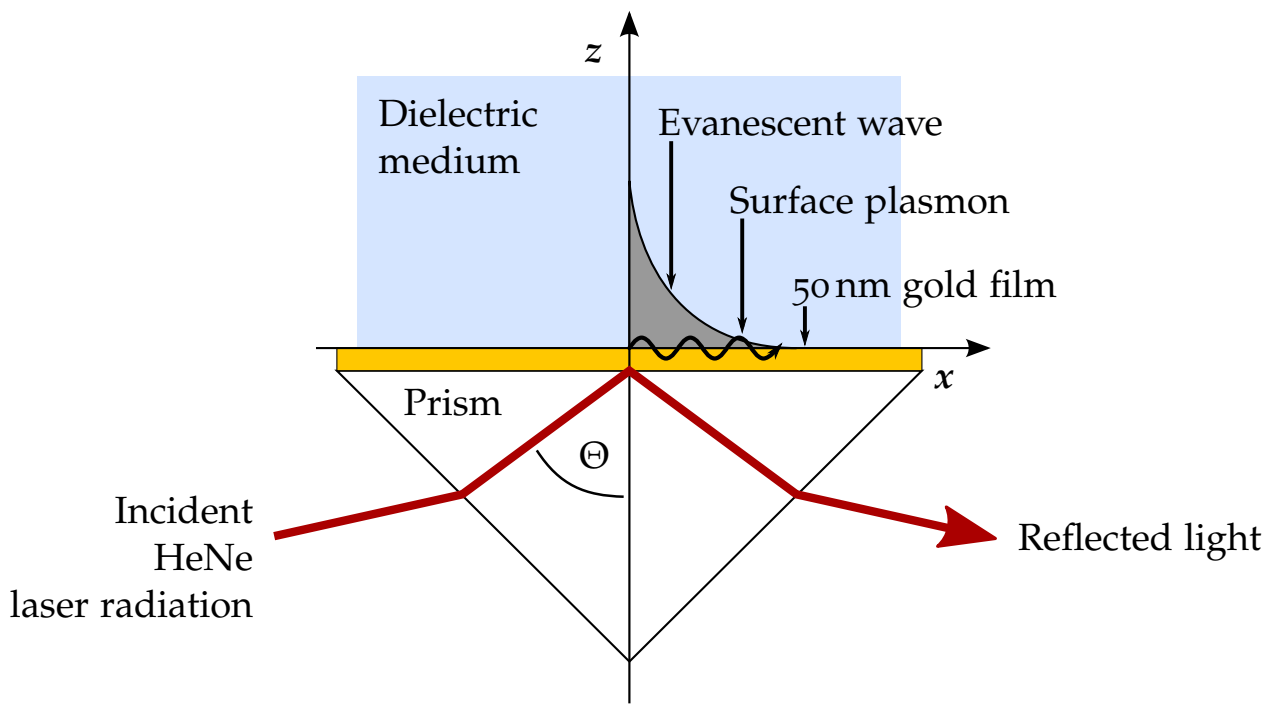

Figure 3.6: SPR setup. Surface plasmon excitation by laser irradiation of an attenuated total internal reflection setup in the Kretschmann configuration.

EXPERIMENTAL SETUP SPR experiments were performed using a Res-Tec2005 spectrometer (Resonant Technologies, Framersheim, Germany). Surface plasmon excitation was achieved employing a HeNe-laser $(632.8 \mathrm{~nm})$ and a LaSFN9 high refractive index prism $(n=1.84)$ with a Kretschmann configuration. A thin gold layer $(50 \mathrm{~nm})$ was evaporated on a LaSFN9 glass substrate. The backside of the glass plate and the prism were optically coupled by a matching immersion oil. An open face flow-cell was mounted on top of the gold surface. In- and outlet tubing provided a flow-through system. The spectrometer was controlled using the WASPLAS software provided by the manufacturer. Before each experiment, sample and detector motors were adjusted by the goniometer stage to align the laser beam path. A gold coated LaSFNg glass plate was functionalized overnight by immersion in a 5:1 ethanolic mixture of HS-11-(EG) $)_{3}-\mathrm{OH}$ and (HS-11-(EG) $)_{3}-\mathrm{NTA}$ ) as described in section 3.2.1.1 and assembled with prism and flow-cell. The NTA head groups were charged with $100 \mathrm{~mm}$ aqueous $\mathrm{Ni}^{2+}$ solution. Before and after protein binding an angular scan $\left(\Theta_{\mathrm{i}}=49^{\circ}\right.$ to $65^{\circ}$ in aqueous solution) was performed in scan mode. Such a spectrum exhibits a steep increase in reflectivity at the critical angle $\Theta_{c}$ upon total internal reflection followed by an abrupt dip indicating the surface plasmon excitation (figure 3.7 A). For kinetic measurements, an angle $\Theta_{\text {kin }}$ in the linear regime before the minimum was chosen. As consequence, reflectivity changes are linearly related to changes in refractive index. Thus, protein binding was monitored in the kinetic mode, that is reflectivity was recorded as a function of time at fixed angle $\Theta_{\text {kin }}$ (figure 3.7 B). 
A

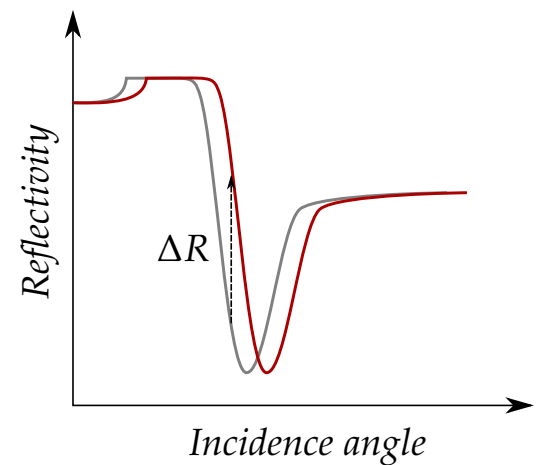

B

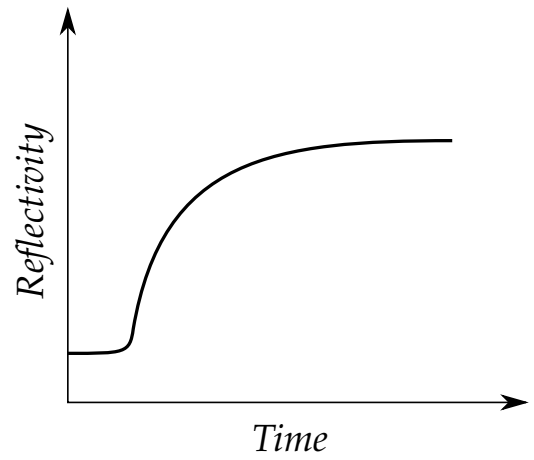

Figure 3.7: (A) Surface plasmon resonance angular reflectivity spectrum in buffer before (gray) and after (red) adsorption. (B) Kinetic measurement of adsorption by monitoring the reflectivity over time at fixed angle $\Theta_{\text {kin }}$.

\subsubsection{Scanning electron microscopy}

The scanning electron microscope (SEM) enables imaging feature sizes of less than $1 \mathrm{~nm}$. In principle, a beam of high energy electrons is emitted by an electron cathode and focused by a series of electromagnetic lenses at the sample's surface. Scanning coils control the beam position and allow the illumination of the sample in a raster scan pattern. The kinetic energy of the incident primary electrons is dissipated upon interaction with the specimen and generates a variety of signals including secondary electrons, backscattered electrons, diffracted backscattered electrons, $X$ rays, and cathodoluminescence. Commonly used for SEM imaging are secondary and backscattered electrons. Morphological and topographical features are illustrated by detecting second electrons, whereas phase contrasts are visualized by the detection of backscattered electrons. To avoid scattering of electrons before reaching the sample and thus reduce the resolution, SEMs are usually operated in high vacuum mode. ${ }^{[79]}$

EXPERIMENTAL SETUP SEM experiments were performed using a LEO SUPRA ${ }^{\mathrm{TM}}$ 35 SEM microscope (Zeiss, Jena, Germany). The microscope is equipped with a Schottky field emission gun, in-lens and lateral secondary electron detectors. Electron acceleration voltages between $2 \mathrm{kV}$ to $5 \mathrm{kV}$ were applied. The aperture was set to $30 \mu \mathrm{m}$. 


\subsection{INTRODUCTION}

The phosphoinositide PIP - A lipid standing apart because of its versatile roles within the cell

Although L- $\alpha$-phosphatidylinositol-4,5-bisphosphate $\left(\mathrm{PIP}_{2}\right)$ constitutes a minor fraction of $\sim_{1} \%$ of the lipids in the plasma membrane, it fulfills a multiplicity of functions within the cell including cytoskeletal attachment, ${ }^{[1]}$ regulation of actin polymerization, ${ }^{[80]}$ enzyme as well as ion channel activation, ${ }^{[81,82]}$ endo- and exocytosis. ${ }^{\left[{ }_{3}-85\right]}$ Moreover, $\mathrm{PIP}_{2}$ is the precursor compound of three second messengers, namely diacylglycerol (DAG), inositol-1,4,5-trisphosphate $\left(\mathrm{IP}_{3}\right)$, and phosphatidylinositol-3,4,5-trisphosphate ( $\left.\mathrm{PIP}_{3}\right)$, thus participating in signal transduction. ${ }^{[86]}$

The most prominent structural feature of $\mathrm{PIP}_{2}$ is the inositol head group, which is rather large compared to usual lipid head groups such as choline (figure 4.1). The $\mathrm{PIP}_{2}$ head group is assumed to protrude from the membrane surface, thus providing membrane anchoring for a vast number of proteins. The orientation relative to the membrane surface is predicted to amount approximately $45^{\circ}$ as derived by molecular dynamic simulations. ${ }^{[87]}$ Owing to its phosphate groups, $\mathrm{PIP}_{2}$ possesses a net negative charge ranging from -3 to -5 under physiological conditions. ${ }^{[88]}$

A

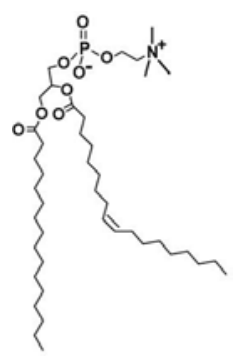

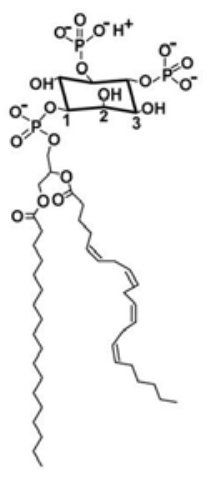

B

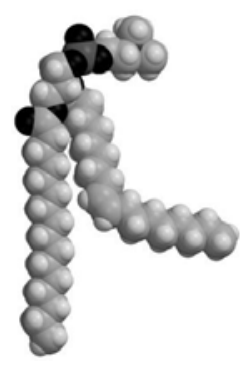

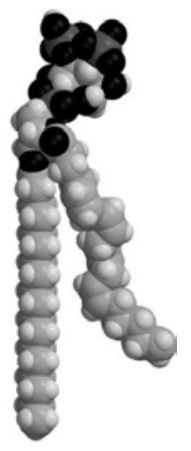

Figure 4.1: A Structure of 1-palmitoyl-2-oleoyl-sn-glycero-3-phosphocholine (POPC) and L- $\alpha$-phosphatidylinositol-4,5-bisphosphate ( $\mathrm{PIP}_{2}$, predominant species). B Space-filling models of POPC and PIP 2 illustrating the differently sized head groups. Adapted from McLaughlin et al. ${ }^{[88]}$

To illustrate the versatility of this particular lipid, we will consider the effect of a local increase in $\mathrm{PIP}_{2}$ concentration on the activation and inhibition of selected 
actin binding proteins, respectively (figure 4.2). On the one hand, binding to $\mathrm{PIP}_{2}$ promotes the function of certain actin binding proteins: (i) Members of the ezrin, radixin, moesin (ERM) protein family are well-known linker proteins, mediating the interaction between the plasma membrane and actin cytoskeleton. Their activation relies on $\mathrm{PIP}_{2}$ binding and subsequent phosphorylation of a conserved threonine residue. The activation and its impact on the F-actin (filamentous actin) binding capability of these proteins will be discussed in chapter 5 and 6 in more detail. (ii) The actin related protein complex 2/3 (Arp 2/3) is an actin filament nucleating protein, thus promoting the formation of a nucleation core, which is the initial step in actin polymerization. Arp 2/3 is stimulated by members of the Wiskott-aldrich syndrome protein (WASP) family, such as neuronal WASP (NWASP), that are themselves activated by $\mathrm{PIP}_{2} \cdot{ }^{[89,90]}$

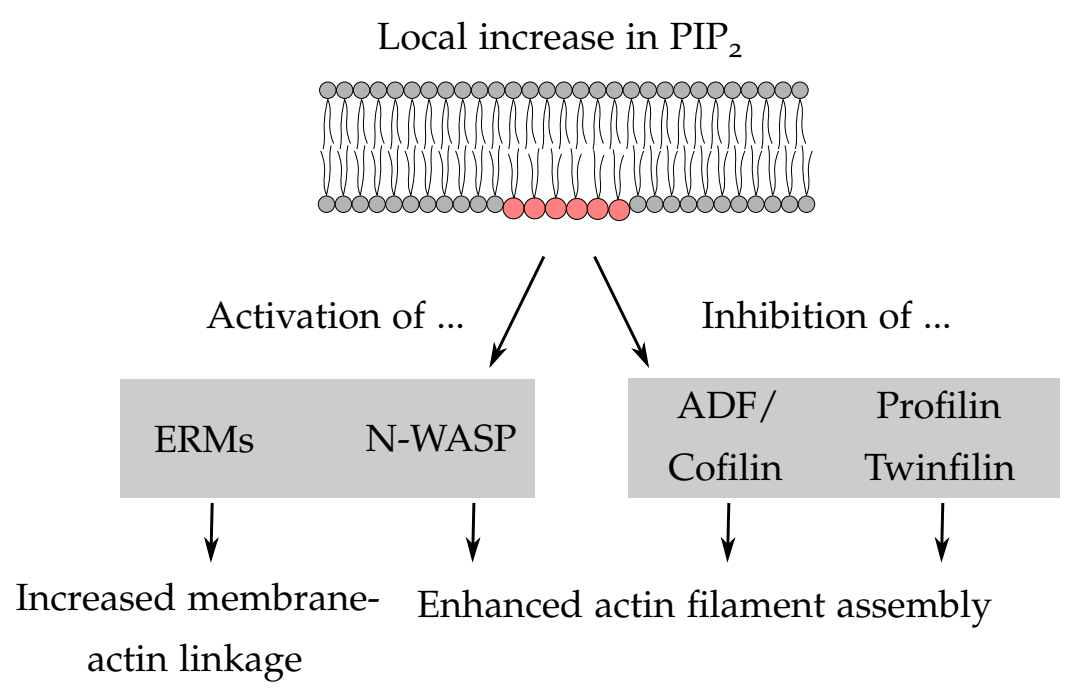

Figure 4.2: Local increase in $\mathrm{PIP}_{2}$ regulates actin binding proteins. ERM proteins are activated, resulting in increased linkage of membrane and actin cytoskeleton. Binding of NWASP to Arp2/3 is enhanced by PIP 2 and promotes actin filament assembly. ADF/Cofilin, Profilin and Twinfilin are inhibited by $\mathrm{PIP}_{2}$ which enhances actin filament assembly as well.

On the other hand, $\mathrm{PIP}_{2}$ binding leads to inhibition of actin binding proteins with severing or capping functions: (i) Actin depolymerizing factor (ADF)/cofilins accelerate actin dynamics by facilitating the disassembly of F-actin. Binding to $\mathrm{PIP}_{2}$

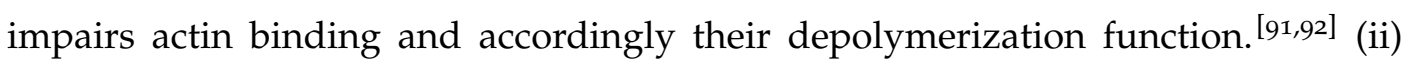
Both profilin and twinfilin are able to sequester actin monomers. However, their function is inhibited upon interaction with $\mathrm{PIP}_{2} \cdot{ }^{\left[{ }^{[93}\right]}$ Based on the ability of PIP $_{2}$ to interact with many actin binding proteins, a pivotal role as regulator of cell shape and dynamic membrane functions has been attributed to this lipid. This view is corroborated by Raucher et al. in optical tweezer experiments, showing that the adhesion energy between plasma membrane and cytoskeleton is directly proportional to the $\mathrm{PIP}_{2}$ concentration. ${ }^{[80]}$ The authors suggested that local changes in 
$\mathrm{PIP}_{2}$ concentration alter the interactions between actin binding proteins, regulating the membrane-cytoskeleton interface.

Considering the variety of functions $\mathrm{PIP}_{2}$ fulfills, the question arises how this rather simple molecule achieves such versatility. Moreover, with regard to the overall scarceness of $\mathrm{PIP}_{2}$ within the membrane, it appears rather astonishing how $\mathrm{PIP}_{2}$ chooses among the numerous $\mathrm{PIP}_{2}$ binding proteins. It has been argued that different $\mathrm{PIP}_{2}$ subpopulations of distinct reactivities owing to its molecular organization - individual lipid, small aggregates or large lipid complexes - might be the key to such diverse functions. ${ }^{[94]}$ Lines of evidence such as the partial inaccessibility of $\mathrm{PIP}_{2}$ moieties toward a $\mathrm{PIP}_{2}$ binding $\mathrm{PH}$ domain support this hypothesis. ${ }^{[95]}$ Therefore, several mechanisms including localized production of $\mathrm{PIP}_{2}$, local sequestration as well as lipid reorganization induced by membrane curvature and cationic or transmembrane proteins have been suggested to account for different $\mathrm{PIP}_{2}$ pools. ${ }^{[96]}$

For instance, local sequestration of $\mathrm{PIP}_{2}$ is achieved by the positively charged myristoylated alanine-rich C-kinase substrate (MARCKS). Both in vivo and in vitro studies suggest that MARCKS is involved in controlling the concentration of free $\mathrm{PIP}_{2}$ within the plasma membrane by nonspecific electrostatic sequestration. [97] Several in vitro experiments have shown that MARCKS is able to efficiently prevent phospholipase $\mathrm{C}$ catalyzed $\mathrm{PIP}_{2}$ hydrolysis, thus corroborating lateral sequestration of $\mathrm{PIP}_{2} \cdot{ }^{[98,99]}$ A mechanism combining both hydrophobic insertion of the myristate anchor and electrostatic interaction with acidic lipids, in particular with $\mathrm{PIP}_{2}$, for localization at the plasma membrane has been proposed to account for the high affinity of MARCKS to $\mathrm{PIP}_{2} \cdot{ }^{[99,100]}$ Furthermore, recent biophysical studies have shown that sequestration of $\mathrm{PIP}_{2}$ can be induced as well by divalent ions such as calcium $\left(\mathrm{Ca}^{2+}\right)$ and magnesium $\left(\mathrm{Mg}^{2+}\right) \cdot{ }^{[101-103]}$ Levental et al. investigated the influence of $\mathrm{Ca}^{2+}$ on domain formation in $\mathrm{PIP}_{2}$-containing monolayers. The authors reported the formation of $\mathrm{PIP}_{2}$ enriched domains with a concomitant surface pressure drop and attributed this effect to the multivalency of $\mathrm{PIP}_{2}$ as a correlation with the $\mathrm{pH}$ value was observed. ${ }^{[102]}$ The idea of a primarily electrostatic mechanism was further corroborated by Ellenbroek et al., showing in experiments and coarse-grained simulations that $\mathrm{PIP}_{2}$ clusters - even at low molar fractions of $2 \%-$ via $\mathrm{Ca}^{2+}$ mediated electrostatic interactions. ${ }^{[103]}$ To what extent $\mathrm{Ca}^{2+}$ contributes to the formation of different $\mathrm{PIP}_{2}$ subpopulations in vivo remains elusive. It is probably a function of the $\mathrm{Ca}^{2+}$ concentration within the cell, which is between ${ }^{10^{-7}} \mathrm{M}$ to ${ }^{10^{-4}}$ M. ${ }^{[104]}$ However, with regard to in vitro studies it is pivotal to carefully evaluate the influence of $\mathrm{Ca}^{2+}$ on $\mathrm{PIP}_{2}$, thus preventing possible misinterpretation as it is often used as spreading additive or in conjunction with $\mathrm{Ca}^{2+}$ dependent proteins. 
How to favor the formation of solid-supported lipid bilayers

In general, many different factors are important with regard to bilayer formation upon fusion of small unilamellar vesicles (SUVs) including ionic strength, vesicle size and composition, surrounding $\mathrm{pH}$ value and surface charge. ${ }^{[05]}$ The forces acting between lipid vesicles and the solid support encompass on the one hand attractive van der Waals (VdW) forces and electrostatic repulsion, which can be described in the framework of the Derjaguin-Landau-Verwey-Overbeek (DLVO) theory. On the other hand, further repulsive contributions such as hydration and steric forces are often predominant at short distances between $1 \mathrm{~nm}$ to $3 \mathrm{~nm}$, thus preventing adhesion and fusion of uncharged lipid vesicles and bilayers. ${ }^{[46,106]}$ In this work, SUVs composed of POPC/PIP 2 (92:8) were used to prepare solid-supported lipid bilayers (SLBs) on silicon substrates. The silicon support is covered with a $100 \mathrm{~nm}$ silicon dioxide layer. Ong et al. suggested a two site model of the silicon dioxide/aqueous interface based on second harmonic generation experiments. ${ }^{[107]}$ One silanol site occupies $81 \%$ of the overall silanol sites and has a $\mathrm{pK}_{\mathrm{a}}$ of 8.5 , whereas the other one, occupying the remaining $19 \%$ of sites, has a $\mathrm{pK}_{\mathrm{a}}$ of 4.5 . Depending on the buffer conditions, $\mathrm{PIP}_{2}$ can have a net charge up to -5 due to its three phosphate groups. The $\mathrm{pK}_{\mathrm{a} 2}$ values of the phosphate groups at the 4- and 5-position of the inositol ring are approximately 6.7 and 7.7. ${ }^{[108]}$ Accordingly, both lipid vesicles and the substrate are negatively charged over a wide range of $\mathrm{pH}$ values. The formation of SLBs involves vesicle adsorption to the substrate followed by vesicle rupture and fusion. ${ }^{[109]}$ Therefore, strategies have been developed minimizing electrostatic repulsion between lipid vesicles and the substrate by either screening or reducing the surface charge.

Screening of surface charges is in general achieved by increasing the ionic strength. On the one hand, monovalent cations such as sodium $\left(\mathrm{Na}^{+}\right)$and potassium $\left(\mathrm{K}^{+}\right)$ adsorb to the negatively charged moieties, thus reducing the Debye length and leading to the formation of an uniform bilayer. ${ }^{[110]}$ Furthermore, it has been shown that $\mathrm{Na}^{+}$binds more strongly to the phosphate moieties of phospholipids than $\mathrm{K}^{+}$ does. ${ }^{[11]}$ On the other hand, divalent cations do not only screen charges, but also appear to interact directly with lipids and surfaces, promoting the fusion of lipid vesicles to solid support. ${ }^{[109,112]}$ In particular $\mathrm{Ca}^{2+}$ influences the spreading process as such that its effect cannot be described adequately by the classical DLVO theory. ${ }^{[106,113]}$ So far, no exact mechanism is known how the $\mathrm{Ca}^{2+}$-promoted SLB formation occurs. During the initial phase of vesicle adsorption, $\mathrm{Ca}^{2+}$ may act as bridging medium between surface and vesicles. ${ }^{[14]}$ Moreover, local dehydration and defects in lipid packing evoked by $\mathrm{Ca}^{2+}$ are suggested. ${ }^{[106]}$ Local stress in- 
duced by $\mathrm{Ca}^{2+}$ may lead to an increased exposure of the hydrophobic core, giving rise to enhanced hydrophobic attraction and hence bilayer fusion. ${ }^{[46]}$

Reducing the surface charge is achieved by lowering the $\mathrm{pH}$ value resulting in increasing protonation of negatively charged moieties. ${ }^{\left[{ }^{13}\right]}$ Within $\mathrm{pH} 6.8$ to $\mathrm{pH} 7.7$, the net charge of $\mathrm{PIP}_{2}$ is calculated to be -4 , whereas it is reduced to approximately -3 at $\mathrm{pH} 4.8 \cdot{ }^{[88,115]}$

In general, in vivo experiments investigating the impact of $\mathrm{PIP}_{2}$ at the plasma membrane are rather challenging as the $\mathrm{PIP}_{2}$ metabolism is controlled by a complex network of enzymatic processes. ${ }^{[16]}$ Unraveling such complexity can be achieved by establishing model systems of known composition. In particular, artificial membrane systems have emerged as versatile tools to study membrane related biological processes in a well defined environment, while retaining important cell membrane properties such as the two-dimensional fluidity and hydrophobic core. Several biophysical studies employing for instance solid-supported membranes (SSMs), ${ }^{[14,15,117]}$ large unilamellar vesicles (LUVs), ${ }^{[118-120]}$ and giant unilamellar vesicles (GUVs), ${ }^{[101,121]}$ aimed at elucidating interactions between proteins and PIP $_{2}$ in a well defined environment. Considering dimensions and membrane curvature, micrometer sized GUVs rather mimic cells than LUVs ( $\sim 100 \mathrm{~nm}$ in diameter) and are easily investigated by means of fluorescence microscopy. ${ }^{[101]}$ Although SSMs are characterized by a reduced lateral lipid mobility because of the solid support and by the lack of an internal compartment as compared to unilamellar vesicles, they are rather stable. ${ }^{[122]}$ Moreover, SSMs are inestimable tools for the investigation of biological processes at the interface between membrane and aqueous phase as they allow beside fluorescence microscopic assays the application of numerous surface sensitive techniques including atomic force microscopy (AFM), reflectometric interference spectroscopy (RIfS), and surface plasmon resonance spectroscopy (SPR).

In this work, we employed two different types of SSMs. On the one hand, we prepared solid-supported lipid bilayers (SLBs) on silicon support. On the other hand, solid-supported hybrid membranes (SHMs) were formed upon spreading of SUVs on silanized silicon substrates. Assuming that at least some in vivo interactions with $\mathrm{PIP}_{2}$ rely on higher concentrations than the global $\mathrm{PIP}_{2}$ level of approximately $1 \%$, the development of model systems with high $\mathrm{PIP}_{2}$ content is highly desirable. However, spreading of SUVs containing $>4 \mathrm{~mol} \% \mathrm{PIP}_{2}$ on silicon support is hindered because of the high net negative charge of $\mathrm{PIP}_{2}$ of approximately -4 at $\mathrm{pH} 7 \cdot 4$. Based on the strategies mentioned above to improve SLB formation, 
three different spreading protocols for $\mathrm{PIP}_{2}$-containing SUVs (up to $8 \mathrm{~mol} \%$ ) were established, addressing either the aspect of screening or reducing surface charges. In presence of either $2 \mathrm{mM} \mathrm{Ca}^{2+}$, or $200 \mathrm{mM} \mathrm{Na}^{+}$, or at $\mathrm{pH} 4.8$, successful SLB formation was monitored by RIfS and confocal laser scanning microscopy (CLSM). Although we succeeded in membrane formation for all spreading protocols, we observed drastic differences with regard to the accessibility of $\mathrm{PIP}_{2}$ head group, which was controlled upon addition of the $\mathrm{PIP}_{2}$ binding protein ezrin.

Moreover, we investigated the distribution of $\mathrm{PIP}_{2}$ within SLBs and SHMs by using the fluorescently labeled $\mathrm{PIP}_{2}$ analogue Bodipy TMR-PIP ${ }_{2}$ as tracer. The lateral mobility of Bodipy TMR-PIP ${ }_{2}$ was monitored by means of fluorescence recovery after photobleaching (FRAP) experiments. Notably, differences regarding the immobile fractions were observed upon comparison of SLBs and SHMs. Furthermore, the impact of clustering agents as well as the influence of $\mathrm{PIP}_{2}$ binding proteins on the lateral mobility was analyzed by using $\mathrm{Ca}^{2+}$ and ezrin, respectively.

\subsection{RESULTS}

4.2.1 Spreading of PIP ${ }_{2}$-containing POPC vesicles ( $8 \mathrm{~mol}_{\mathrm{O}} \mathrm{PIP} \mathrm{P}_{2}$ ) on silicon substrates and subsequent ezrin binding

Three spreading protocols, namely A, B, and C were evaluated (table 4.1). RIfS was used as a precise tool to monitor the change in optical thickness $(\Delta O T)$ during the spreading process of SUVs composed of POPC/PIP 2 (92:8) on hydrophilized silicon substrates.

Table 4.1: Spreading protocols A, B, and C for $\mathrm{PIP}_{2}$-containing SUVs.

\begin{tabular}{|c|c|}
\hline Buffer & Composition \\
\hline A & $20 \mathrm{~mm}$ TRIS/ $\mathrm{HCl}, 2 \mathrm{mM} \mathrm{CaCl}_{2}, 50 \mathrm{~mm} \mathrm{KCl}, 0.1 \mathrm{~mm} \mathrm{NaN}_{3}, \mathrm{pH} 7.4$ \\
\hline $\mathrm{AE}$ & $20 \mathrm{~mm}$ TRIS/HCl, $50 \mathrm{~mm} \mathrm{KCl,} 10 \mathrm{~mm}$ EGTA, o.1 mM NaN ${ }_{3}, \mathrm{pH} 7.4$ \\
\hline B & $20 \mathrm{~mm}$ TRIS/HCl, $200 \mathrm{~mm} \mathrm{NaCl}$, o.1 mM $\mathrm{NaN}_{3}, \mathrm{pH} 7.4$ \\
\hline C & $20 \mathrm{~mm}$ Citrate, 50 mM KCl, o.1 mM EDTA, o.1 $\mathrm{mm} \mathrm{NaN}_{3}$, pH 4.8 \\
\hline E1 & $20 \mathrm{~mm}$ TRIS/HCl, $50 \mathrm{~mm} \mathrm{KCl,} \mathrm{o.1} \mathrm{mм} \mathrm{EDTA,} \mathrm{o.1} \mathrm{mm} \mathrm{NaN}$ m, $\mathrm{pH} 7.4$ \\
\hline
\end{tabular}

After membrane formation, ezrin was added to monitor the accessibility of the $\mathrm{PIP}_{2}$ head group. The RIfS experiments were performed according to the procedure described in section 3.3.3.1. The refractive indices of silicon dioxide, lipid bilayers and proteins are in a similar range. ${ }^{\left[7^{0-72}\right]}$ Accordingly, detected changes in OT, averaged over an area of $1 \mathrm{~mm}^{2}$, can be correlated with changing physical layer thicknesses 
upon deposition of lipid or protein material as the optical thickness is the product of refractive index $n$ and physical layer thickness $d$. Figure 4.3 shows the typical course of a RIfS experiment. The addition of $\mathrm{PIP}_{2}$-containing SUVs to the silicon substrate leads to spreading of vesicles and subsequent formation of a SLB $(a, b)$. The process is finished when no more changes in OT are detected, resulting typically in an average increase in $O T$ of approximately $6 \mathrm{~nm}$ (c). The addition of protein generates an additional increase in $O T$ due to adsorption of protein to $\mathrm{PIP}_{2}(\mathrm{~d})$.

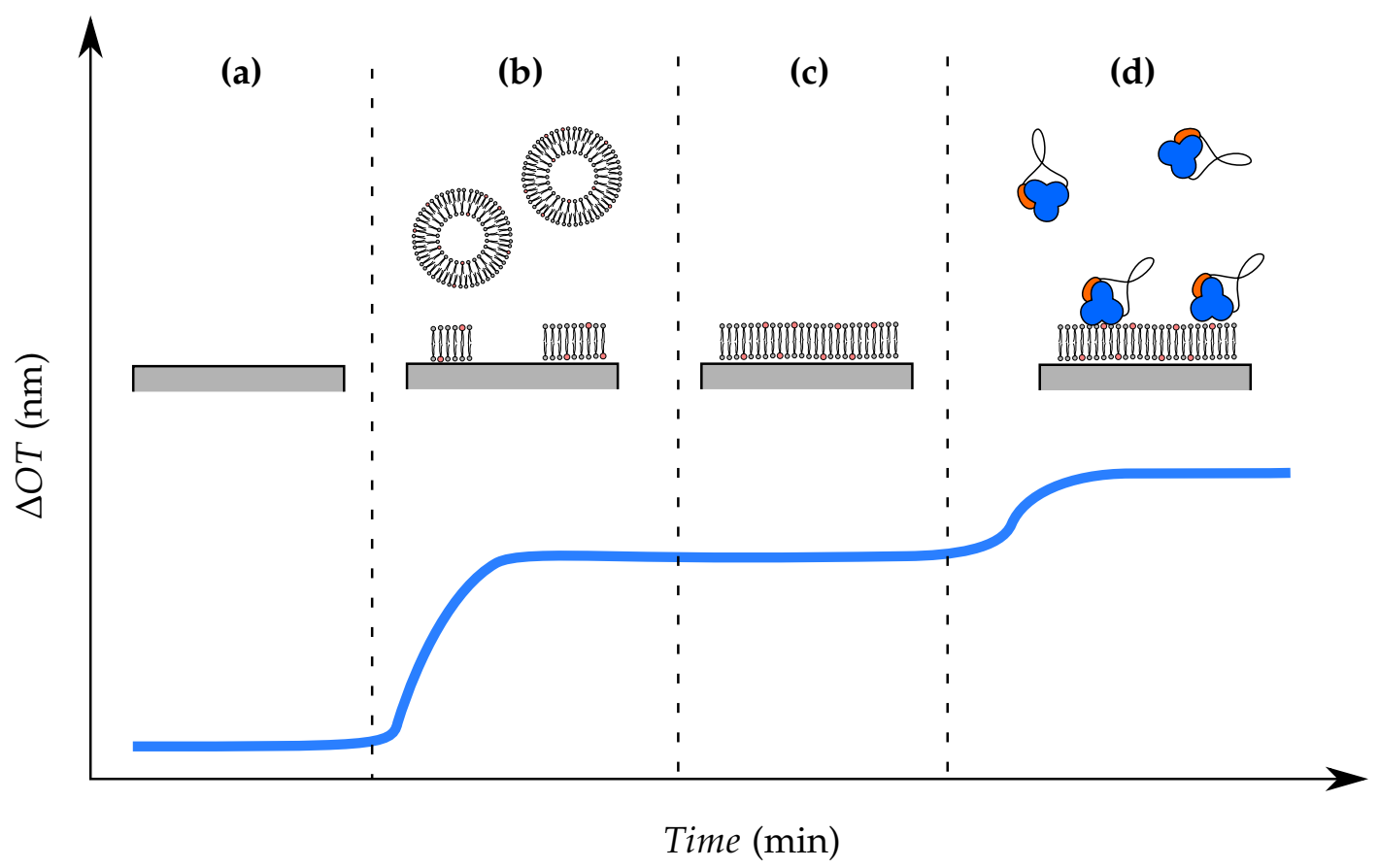

Figure 4.3: Schematic drawing of typical RIfS experiment. Baseline of bare silicon wafer (a). Spreading of $\mathrm{PIP}_{2}$-containing SUVs leads to increasing OT (b). SLB formation is achieved when no more changes in OT are detected (c). Binding of protein to $\mathrm{PIP}_{2}$ leads to further increase in $O T(\mathbf{d})$.

In addition to RIfS experiments, the membrane quality was evaluated by CLSM imaging. SLBs consisting of POPC/PIP 2 / Perylene (91:8:1) were prepared according to the respective spreading protocol. AlexaFluor488 labeled ezrin was added to visualize the accessibility of the $\mathrm{PIP}_{2}$ head group to ezrin binding depending on the spreading conditions with higher spatial resolution as compared to RIfS. In a previous thesis, it has been shown that the fluorescent label does not change the functionality of ezrin. ${ }^{[123]}$

Protocol A The spreading of SUVs in $\mathrm{Ca}^{2+}$-containing buffer A led to an increase in optical thickness of $\triangle O T=10.2 \mathrm{~nm}$. By rinsing with EGTA-containing buffer $\mathrm{AE}$ to remove $\mathrm{Ca}^{2+}$ from the system, a decrease of $\triangle O T=-1.9 \mathrm{~nm}$ was observed, leading to a final value of $\triangle O T=8.3 \mathrm{~nm}$ for the POPC $/ \mathrm{PIP}_{2}$ bilayer (figure 4.4 A1, RIfS). This $\triangle O T$ value was remarkable as typical $\triangle O T$ values due to bilayer formation lay rather in the range of $6 \mathrm{~nm}$ to $6.5 \mathrm{~nm}$ (chapter 5 ). The optical thick- 
ness remained constant while the buffer was changed to protein buffer E1 to set appropriate conditions for ezrin binding to $\mathrm{PIP}_{2}$. The addition of ezrin to a final concentration of $0.7 \mu \mathrm{M}$, however, did not affect the optical thickness (figure 4.4 A2, RIfS). Apparently, no ezrin was bound to the PIP $_{2}$-containing bilayer.

SLBs prepared according to spreading protocol A showed a continuous Perylene fluorescence, indicating the formation of a bilayer (figure 4.4 A1, CLSM). Nevertheless, the fluorescence intensity was not as homogeneous as expected. Bright fluorescent circular structures, which can be attributed to adhered excess vesicles, were distributed over the entire sample. The addition of AlexaFluor488 labeled ezrin (final concentration $0.5 \mu \mathrm{M}$ ) led to the formation of submicrometer clusters rather than to a complete surface coverage (figure 4.4 A2, CLSM).

RIfS

A1

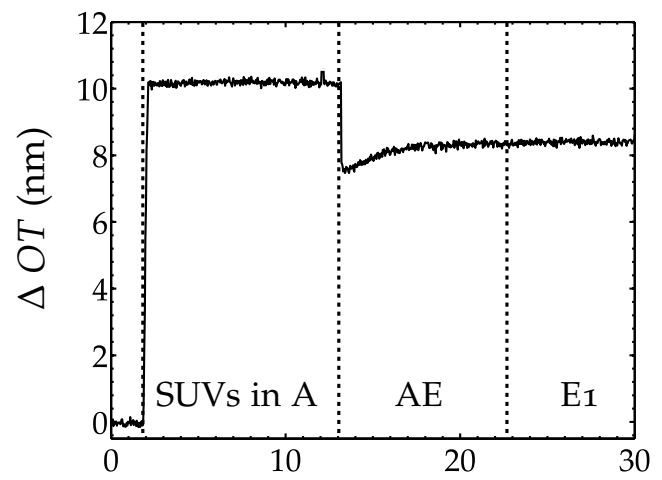

A2

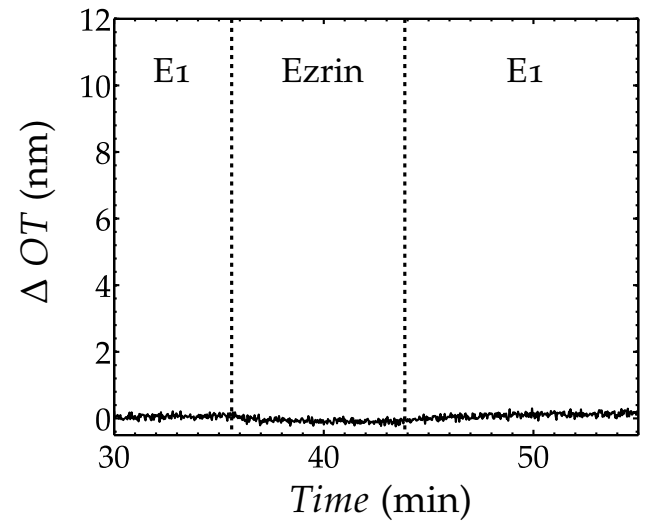

CLSM
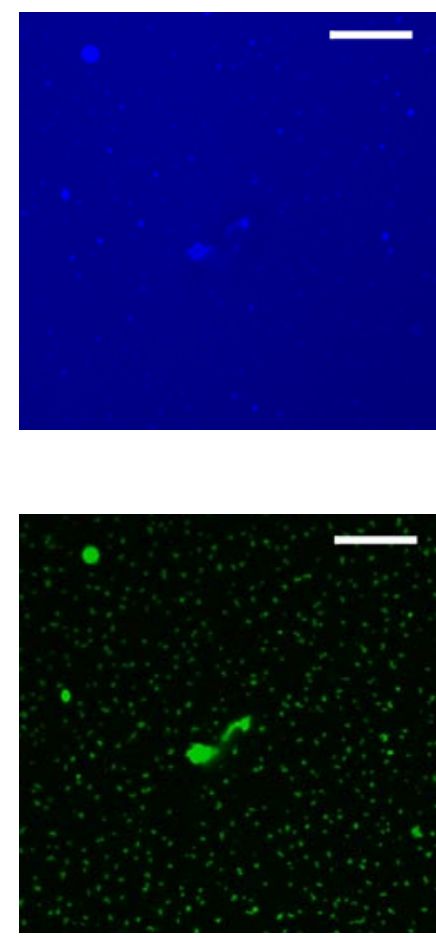

Figure 4.4: Spreading process of SUVs composed of POPC/PIP 2 (92:8) in buffer A, subsequent rinsing with buffer $\mathrm{AE}$ and protein buffer $\mathrm{E}_{1}$ monitored by means of reflectometric interference spectroscopy (A1, RIfS). Addition of ezrin to a final concentration of $0.7 \mu \mathrm{M}$ to $\mathrm{PIP}_{2}$-containing membrane (A2, RIfS; OT was set to zero to allow fast determination of $\triangle O T$ upon protein binding). Confocal fluorescent image of POPC/PIP $2 /$ Perylene (91:8:1) SLB prepared in buffer A (A1, CLSM). After rinsing with AE and E1, AlexaFluor488 labeled ezrin (final concentration $0.5 \mu \mathrm{M})$ was added (A2, CLSM). Scale bars: 10 $\mu \mathrm{m} .^{\mathrm{a}}$

In conclusion, both methods RIFS and CLSM validated the formation of a continuous bilayer via spreading procedure A. However, ezrin binding to the SLB was 
strongly hampered, indicated by the unchanged $\triangle O T$ value in RIfS and by the spotlike ezrin adsorption observed by CLSM.

To evaluate the apparent high $\triangle O T$ due to membrane formation found in RIfS measurements, we performed further CLSM experiments controlling the formed SLB before and after rinsing with $\mathrm{Ca}^{2+}$-chelating buffer. Instead Perylene, the fluorophor Bodipy- $\mathrm{C}_{12} \mathrm{HPC}$ was used as less energetic light is required for its excitation. Therefore, it is less sensitive toward photobleaching. We found that in presence of $\mathrm{Ca}^{2+}$ not only a lipid bilayer is formed by spreading POPC $/ \mathrm{PIP}_{2} /$ Bodipy- $\mathrm{C}_{12} \mathrm{HPC}$ (96.8:3:0.2) vesicles onto silicon substrates, but also a significant number of intact vesicles, adhering to the bilayer, were observed as indicated by the bright green fluorescence (figure 4.5 A). The amount of vesicles was reduced by rinsing with EDTA-containing buffer, although no complete detachment was achieved (figure 4.5 B), similar to the situation depicted in figure 4.4 A1, CLSM.

A

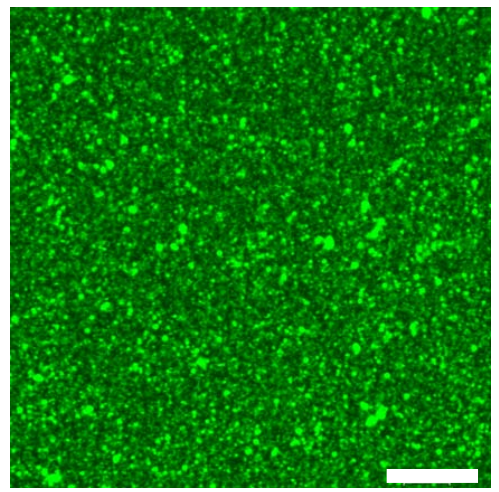

B

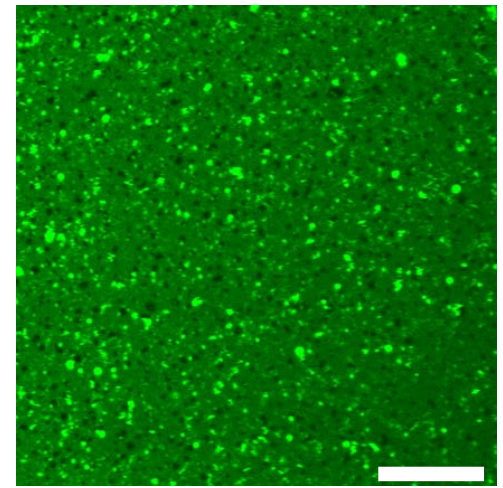

Figure 4.5: Confocal fluorescent image of SLB formed upon spreading of SUVs composed of POPC/PIP $/$ BODIPY-C ${ }_{12} \mathrm{HPC}$ (96.8:3:0.2) in buffer (20 mM TRIS/HCl, $10 \mathrm{~mm}$ $\mathrm{CaCl}_{2}, 50 \mathrm{~mm} \mathrm{KCl}$, o.1 $\mathrm{mM} \mathrm{NaN}_{3}, \mathrm{pH} 7.4$ ) before (A) and after 15 min incubation in EDTAcontaining buffer (B). Scale bars: $10 \mu \mathrm{m}$.

Consequently, the apparent high $\triangle O T$ value obtained for the SLB formation according to spreading protocol A most probably originates in adhering excess vesicle, which are impossible to remove by rinsing with $\mathrm{Ca}^{2+}$-chelating buffer.

PROTOCOL B Spreading SUVs in high salt buffer B caused an increase in optical thickness of $\triangle O T=6.4 \mathrm{~nm}$, indicating the formation of a lipid bilayer. Buffer B was exchanged against protein buffer E1 and a final value of $\triangle O T=6.6 \mathrm{~nm}$ was obtained for the formed bilayer (figure 4.6 B1, RIfS). An increase in optical thickness of $\triangle O T=1.0 \mathrm{~nm}$ was detected after the addition of ezrin to a final concentration of $0.7 \mu \mathrm{M}$, illustrating the adsorption of protein. Rinsing with buffer E1 reduced the protein layer's thickness to $\triangle O T=0.6 \mathrm{~nm}$ (figure $4.6 \mathrm{~B} 2$, RIfS).

SLBs prepared for CLSM experiments according to the spreading protocol B showed a rather homogeneously distributed Perylene fluorescence as compared to the one 
observed with spreading procedure C (figure 4.6 B2, CLSM). After the addition of AlexaFluor 488 labeled ezrin to a final concentration of $0.5 \mu \mathrm{M}$, few protein clusters with a diameter of several micrometers were detectable among many submicrometer clusters (figure $4.6 \mathrm{~B} 2$, CLSM).

RIfS

B1

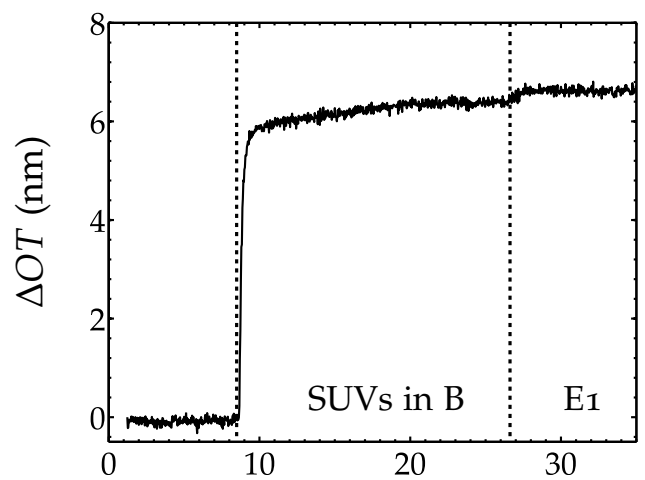

B2

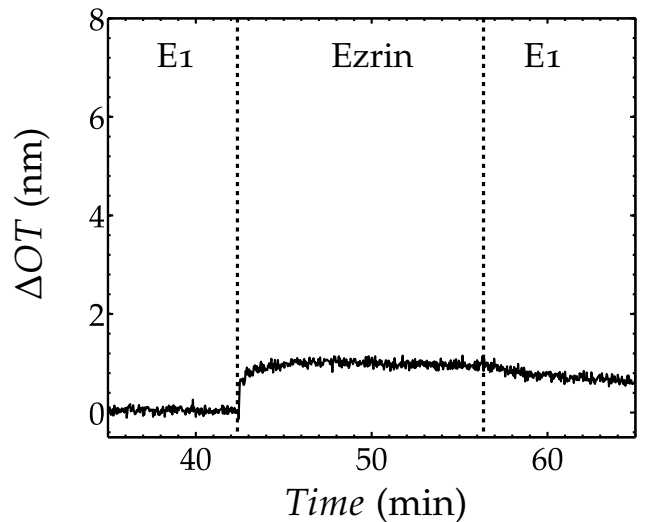

CLSM
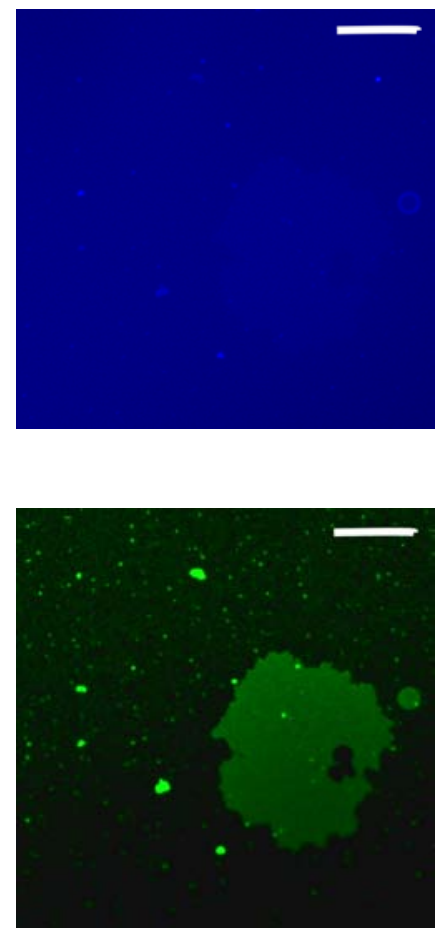

Figure 4.6: Spreading process of SUVs composed of POPC/PIP 2 (92:8) in buffer B, subsequent rinsing with protein buffer $\mathrm{E}_{1}$ monitored by means of reflectometric interference spectroscopy (B1, RIfS). Addition of ezrin to a final concentration of $0.7 \mu \mathrm{M}$ to $\mathrm{PIP}_{2}$ containing membrane (B2, RIfS; OT was set to zero to allow fast determination of $\triangle O T$ upon protein binding). Confocal fluorescent image of POPC/PIP $2 /$ Perylene (91:8:1) SLB prepared in buffer B (B1, CLSM). After rinsing with E1, AlexaFluor488 labeled ezrin (final concentration $0.5 \mu \mathrm{M})$ was added (B2, CLSM). Scale bars: $10 \mu \mathrm{m}$. $^{\mathrm{b}}$

Conclusively, SLB formation according to spreading procedure B was confirmed by RIfS and CLSM. Although some ezrin binding to the SLB was detected, the observed surface coverage did not correspond to full coverage, which we would expect in case of SLBs containing $8 \mathrm{~mol} \% \mathrm{PIP}_{2}{ }^{\left[{ }^{14}\right]}$

PROTOCOL C Upon spreading of SUVs in buffer $\mathrm{C}$ at $\mathrm{pH} 4.8$, an increase in optical thickness of $\triangle O T=6.3 \mathrm{~nm}$ was detected. The buffer exchange to protein buffer E1 did not affect the optical thickness of the formed bilayer (figure 4.7 $C_{1}$, RIfS). In contrary to the spreading protocols $A$ and $B$, the addition of ezrin (final concentration $0.7 \mu \mathrm{M}$ ) led to an increase in optical thickness of $\Delta O T=6.5 \mathrm{~nm}$. Rins-

b RIfS experiments and CLSM imaging performed by C. Kramer and D. Morick. 
ing with buffer E1 removed nonspecifically bound protein and caused a decrease of $\triangle O T=-1.8 \mathrm{~nm}$. The optical thickness of the protein layer was determined with $\Delta O T=4.7 \mathrm{~nm}$ (figure $4.7 \mathrm{C} 2, \mathrm{RIfS}$ ).

Analogous to the procedures A and B, the preparation of SLBs according to spreading protocol $\mathrm{C}$ yielded membranes of good quality, as indicated by the homogeneous Perylene fluorescence (figure $4.7 \mathrm{C}_{1}, \mathrm{CLSM}$ ). Upon addition of AlexaFluor488 labeled ezrin to a final concentration of $0.5 \mu \mathrm{M}$, a complete ezrin layer was obtained, as can be concluded from the abundant AlexaFluor488 fluorescence (figure $4.7 \mathrm{C} 2$, CLSM).

RIfS

C1

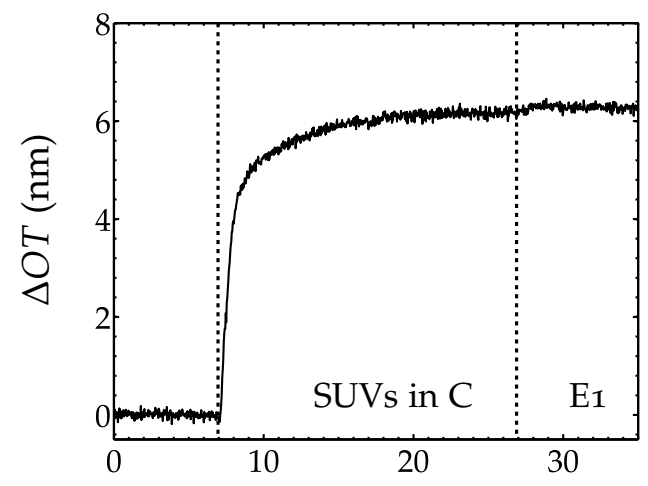

$\mathrm{C} 2$

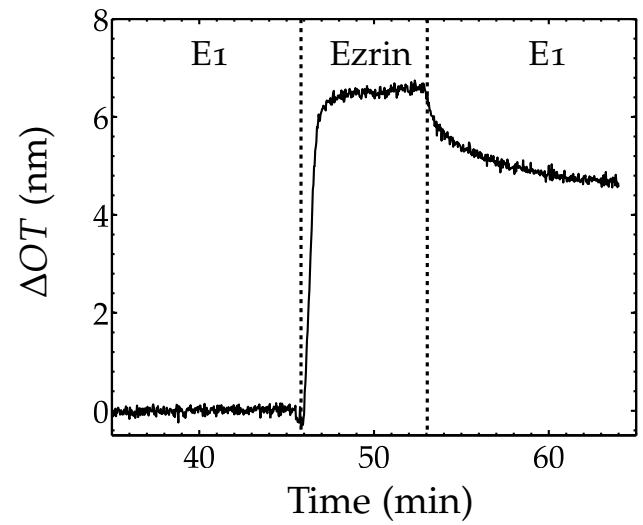

CLSM
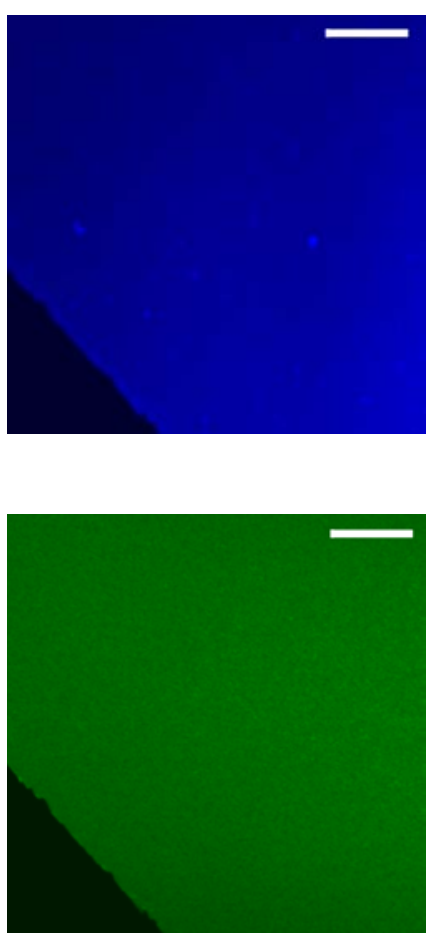

Figure 4.7: Spreading process of SUVs composed of POPC/PIP ${ }_{2}(92: 8)$ in buffer C, subsequent rinsing with protein buffer $\mathrm{E}_{1}$ monitored by means of reflectometric interference spectroscopy (CI, RIfS). Addition of ezrin to a final concentration of $0.7 \mu \mathrm{M}$ to $\mathrm{PIP}_{2^{-}}$ containing membrane (C2, RIfS; OT was set to zero to allow fast determination of $\triangle O T$ upon protein binding). Confocal fluorescent image of POPC/PIP $2 /$ Perylene (91:8:1) SLB prepared in buffer C (C1, CLSM). After rinsing with E1, AlexaFluor488 labeled ezrin (final concentration $0.5 \mu \mathrm{M})$ was added $\left(\mathbf{C}_{2}, \mathbf{C L S M}\right)$. The edge of the substrate is visualized in the lower left corner of the image (intersection to black area). Scale bars: $10 \mu \mathrm{m} .{ }^{\mathrm{C}}$

Notably, spreading protocol C provides both reliable SLB formation and unhindered ezrin binding to $\mathrm{PIP}_{2}$-containing SLBs as indicated by a distinct increase in OT upon ezrin addition and by the homogeneously dispersed AlexaFluor488 fluorescence.

c RIfS experiments and CLSM imaging performed by C. Kramer and D. Morick. 
4.2.2 Lateral mobility of Bodipy TMR-PIP 2 within solid-supported membranes (3 mol\% $\left.P I P_{2}\right)$

Fluorescence recovery after photobleaching (FRAP) experiments were performed to investigate the lateral mobility of the fluorescent $\mathrm{PIP}_{2}$ analogue Bodipy TMR$\mathrm{PIP}_{2}$ within membranes. For this purpose, solid-supported lipid bilayers (SLBs) as well as hybrid membranes (SHMs) on solid-support were analyzed. Membranes were obtained by spreading SUVs composed of POPC/PIP $2 /$ Bodipy TMR$\mathrm{PIP}_{2} /$ Perylene (96:2.5:0.5:1) either on a hydrophilized silicon substrate (SLB) or on dodecyl-trichlorosilane functionalized silicon wafers (SHM). Owing to the lower $\mathrm{PIP}_{2}$ content, spreading could be directly performed in E1 buffer. The lower $\mathrm{PIP}_{2}$ percentage was chosen to visualize a possible reorganization of $\mathrm{PIP}_{2}$ caused by the addition of $\mathrm{PIP}_{2}$ binding agents. Perylene was added to control whether membrane formation was successful, while the distribution of $\mathrm{PIP}_{2}$ and its lateral mobility was monitored by Bodipy TMR-PIP 2 fluorescence. All membranes, SHMs and SLBs, were characterized by a homogeneously distributed Perylene fluorescence similar to the images shown in figures 4.4 to 4.7. Typical pictures recorded during a FRAP experiment are depicted in figure 4.8. The first two of them, 1a and $2 \mathrm{a}$, were taken before the bleach pulse. The Bodipy TMR-PIP ${ }_{2}$ fluorescence is in both cases homogeneously dispersed within the membrane considering the resolution of the CLSM. Small defects are present in $1 \mathrm{a}$, whereas $2 \mathrm{a}$ seems to be more noisy owing to the higher magnification. The series $1 b-d$ and $2 b-d$ illustrate the fluorescence recovery of Bodipy TMR-PIP 2 due to diffusion after bleaching. However, it is obvious that in case of the SHM setup (figure $4.8 \mathrm{Ib}-\mathrm{d}$ ), the fluorescence recovers almost completely within short time, whereas the SLB stays partially bleached (figure $4.82 \mathrm{~b}-\mathrm{d}$ ). More precisely, a partial recovery of fluorescence intensity is observed, while the edges remain rather well-defined.
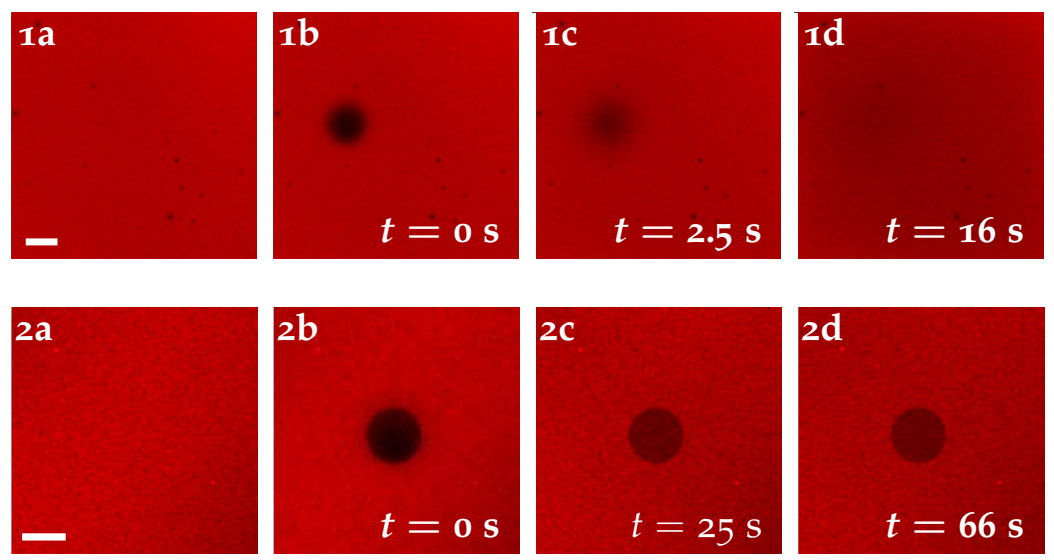

Figure 4.8: Fluorescent recovery experiments on solid-supported hybrid membranes (1ad) and lipid bilayers (2a-d). Lipid composition: POPC $/ \mathrm{PIP}_{2} /$ Bodipy TMR-PIP $2 /$ Perylene 96:2.5:0.5:1. Scale bars: $10 \mu \mathrm{m}$. 
To quantify the differences between SLB and SHM setup, the diffusion coefficients and mobile fractions of Bodipy TMR-PIP 2 were determined according to Jönsson et al. (section 3.3.2.1). ${ }^{[65]}$ In case of the SLB setup, the diffusion coefficient was in the range of $(2.9 \pm 0.3) \mathrm{\mu m}^{2} / \mathrm{s}$ (figure $4.9 \mathrm{~A}$, dark blue). The diffusion coefficient determined for the SHM setup amounts to $(3.0 \pm 0.5) \mathrm{\mu m}^{2} / \mathrm{s}$ (figure $4.9 \mathrm{~A}$, light blue), which is similar to that of the SLB setup. Differences between the setups become evident by comparing the respective mobile fractions. The SLB setup is characterized by a reduced mobile fraction of $(52 \pm 9) \%$ (figure $4.9 \mathrm{~B}$, dark blue), whereas SHMs seem to recover almost completely with ( $98 \pm 3) \%$ (figure $4.9 \mathrm{~B}$, light blue). The calculation is based on ten and eleven fluorescence recovery measurements from four independent experiments for the SLB and SHM setup, respectively.

A

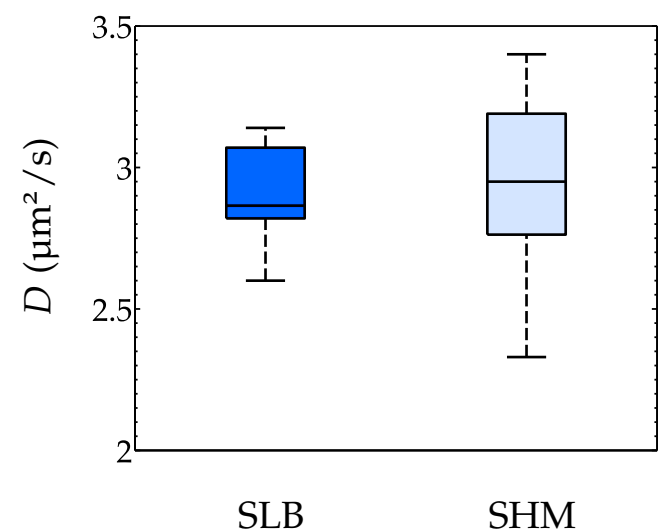

B

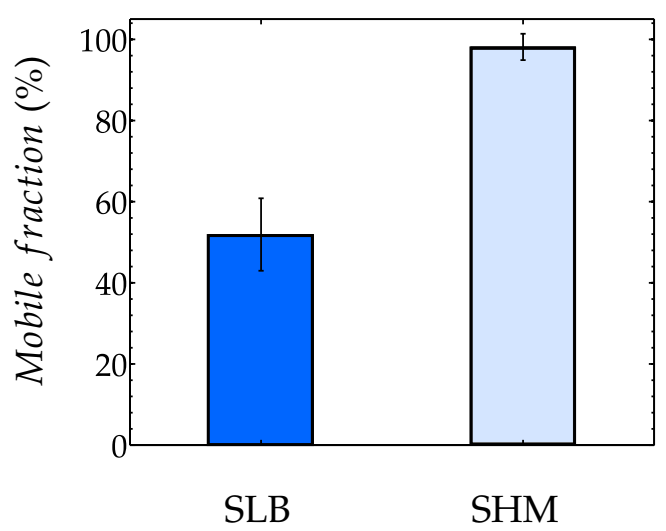

Figure 4.9: Diffusion coefficients visualized as box plots (A) and bar diagram showing the mobile fractions (B) of Bodipy TMR-PIP ${ }_{2}$ within SLBs (dark blue, $n=10$ from 4 independent experiments) and SHMs (light blue, $n=11$ from 4 independent experiments) obtained by means of FRAP. Lipid composition of membranes: POPC $/ \mathrm{PIP}_{2} /$ Bodipy TMR$\mathrm{PIP}_{2} /$ Perylene (96:2.5:0.5:1).

Due to its excellent fluorescence recovery behavior, the SHM setup was chosen to investigate the influence of divalent ions as well as the effect of protein binding on the lateral mobility of Bodipy TMR-PIP ${ }_{2}$. After a $2 \mathrm{~h}$ incubation period with $\mathrm{Ca}^{2+}$-containing buffer (20 mM TRIS/ $\mathrm{HCl}, 50 \mathrm{~mm} \mathrm{CaCl}$, $50 \mathrm{~mm} \mathrm{KCl}$, 0.1 mM EDTA, o.1 $\mathrm{mM} \mathrm{NaN}_{3}, \mathrm{pH} 7.4$ ), the diffusion coefficient of Bodipy TMR-PIP 2 decreased to $(1.4 \pm 0.4) \mu \mathrm{m}^{2} / \mathrm{s}$ (figure 4.10, light red). The addition of AlexaFluor488 labeled ezrin to a final concentration of $0.5 \mu \mathrm{M}$ to SHMs, however, had even a more drastic effect on the lateral diffusion of Bodipy TMR-PIP ${ }_{2}$. After a period of $2 \mathrm{~h}$ protein incubation, it was reduced to $(0.7 \pm 0.5) \mu \mathrm{m}^{2} / \mathrm{s}$ (figure 4.10 , light green). No diffusion coefficient could be determined for AlexaFluor488 labeled ezrin, as it appeared to be completely immobile during the measurements. Only regions characterized by an apparently homogeneous distribution of Bodipy TMR-PIP ${ }_{2}$ fluorescence were evaluated by means of FRAP. 


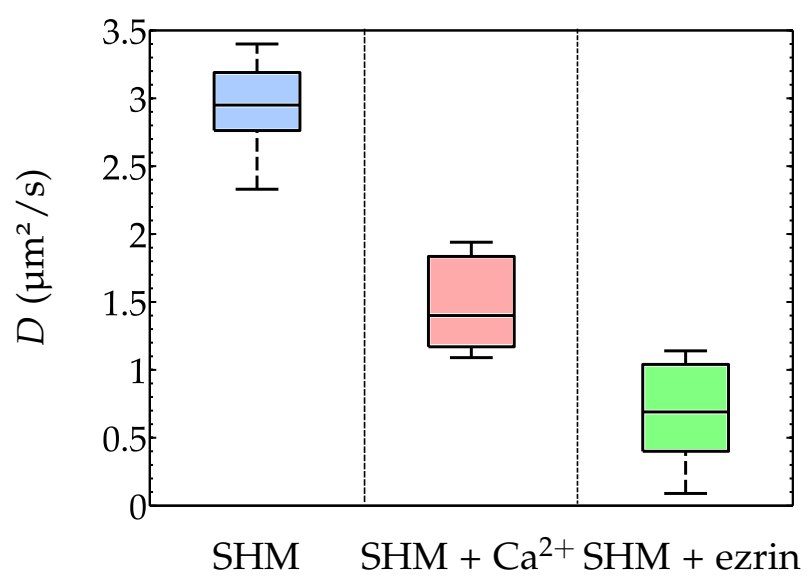

Figure 4.10: Box plots showing the distribution of diffusion coefficient of Bodipy TMR-PIP within SHMs (POPC $/ \mathrm{PIP}_{2} /$ Bodipy TMR-PIP 2 / Perylene 96:2.5:0.5:1) without additive (light blue, $n=11$ from 4 independent experiments) and after the addition of either $50 \mathrm{~mm} \mathrm{Ca}^{2+}$ (light red, $n=8$ from 2 independent experiments) or $0.5 \mu \mathrm{M}$ AlexaFluor 488 labeled ezrin (light green, $n=14$ from 5 independent experiments) obtained by means of FRAP.

Figure 4.11 shows the respective mobile fractions. The mobile fraction of the SHM system without additive amounts to $(98 \pm 3) \%$ (light blue), whereas the addition of $\mathrm{Ca}^{2+}$ led to $(85 \pm 13) \%$. In presence of ezrin, the mobile fraction was reduced to $(89 \pm 9) \%$. Within the range of error $\mathrm{PIP}_{2}$ is still rather mobile, indicating the formation of small $\mathrm{PIP}_{2}$ aggregates diffusing more slowly than the individual lipid, however.

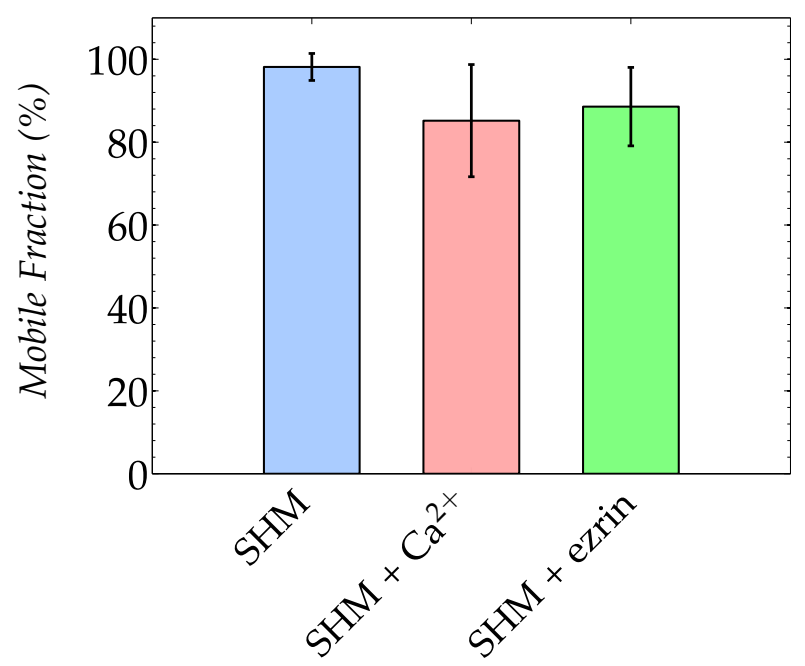

Figure 4.11: Bar diagram showing the mobile fractions of Bodipy TMR-PIP ${ }_{2}$ within SHMs (POPC $/ \mathrm{PIP}_{2} /$ Bodipy TMR-PIP 2 /Perylene 96:2.5:0.5:1) without additive (light blue, $n=11$ from 4 independent experiments) and after the addition of either $50 \mathrm{~mm} \mathrm{Ca}^{2+}$ (light red, $n=8$ from 2 independent experiments) or $0.5 \mu \mathrm{M}$ AlexaFluor 488 labeled ezrin (light green, $n=14$ from 5 independent experiments) obtained by means of FRAP.

Considering that AlexaFluor488 labeled ezrin was immobile during the measurement, the rather small reduction in mobile fraction implicates that the interaction between $\mathrm{PIP}_{2}$ and ezrin might not be static. Instead, dissociation of $\mathrm{PIP}_{2}$ and rebinding of $\mathrm{PIP}_{2}$ can occur. As AlexaFluor488 labeled ezrin remained bound to the 
surface, we can assume that the protein is at least bound via two different $\mathrm{PIP}_{2}$ binding sites. This is in agreement with Niggli et al. proposing several distinct $\mathrm{PIP}_{2}$ binding based on site directed mutagenesis. ${ }^{[124]}$

4.2.3 Influence of additives on Bodipy TMR-PIP ${ }_{2}$ distribution within solid-supported hy-

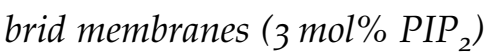

In the previous section we described how additives had affected the lateral mobility of Bodipy TMR-PIP ${ }_{2}$. These results raise the question whether the distribution of Bodipy TMR-PIP 2 is changed by them. Is their effect observable within the resolution of the CLSM or are the structures too small to be resolved? Confocal fluorescent images were taken before the addition of either $\mathrm{Ca}^{2+}$ or ezrin to control the formed SHM. The Bodipy TMR-PIP ${ }_{2}$ as well as the Perylene fluorescence was in general homogeneously dispersed (figure $4.12 \mathrm{a}, \mathrm{b}$ ). Some inhomogeneities in the fluorescence intensity of Bodipy TMR-PIP ${ }_{2}$ could be observed remaining unchanged on the time scale of the experiment, however (figure $4.12 \mathrm{~b}$ ).

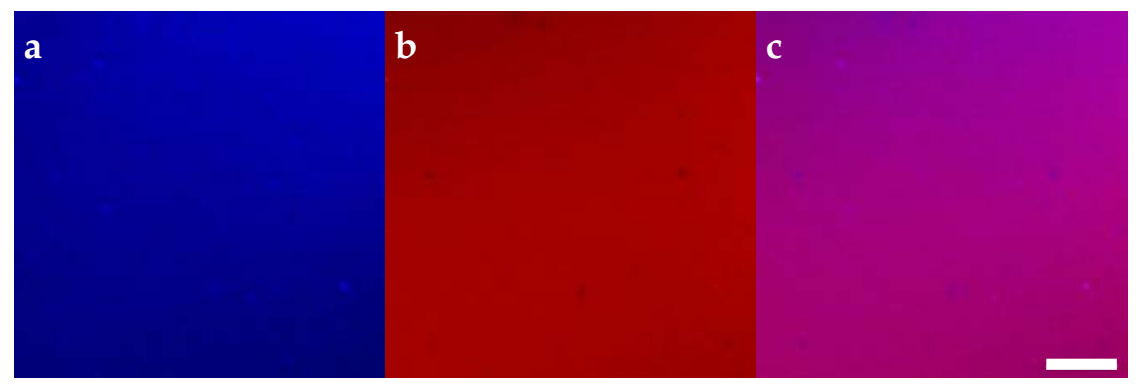

Figure 4.12: Confocal fluorescent image of SHM composed of POPC/PIP 2 /Bodipy TMR$\mathrm{PIP}_{2} /$ Perylene (96:2.5:0.5:1). (a) blue channel: Perylene, (b) red channel: Bodipy TMR-PIP ${ }_{2}$, (c) overlay. Scale bar: $10 \mu \mathrm{m}$.

After a $2 \mathrm{~h}$ incubation period in $\mathrm{Ca}^{2+}$-containing buffer $(20 \mathrm{~mm}$ TRIS/ $\mathrm{HCl}, 50 \mathrm{~mm}$ $\mathrm{CaCl}_{2}, 50 \mathrm{~mm} \mathrm{KCl}$, o.1 mM $\mathrm{NaN}_{3}, \mathrm{pH} 7.4$ ) and subsequent rinsing with EDTA-free E1 buffer, the Perylene fluorescence was still uniformly distributed (figure 4.13 a), whereas inhomogeneities in the Bodipy TMR-PIP ${ }_{2}$ fluorescence were observed (figure $4.13 \mathrm{~b}$ ). It appears that the Bodipy TMR-PIP 2 fluorophor is excluded from micrometer sized areas within the hybrid membrane. This behavior, tough, was not observed for the total membrane surface area, but rather a coarsening of the Bodipy TMR-PIP ${ }_{2}$ fluorescence was detectable. The overlay of Perylene and Bodipy TMR-PIP ${ }_{2}$ channel (figure $4.13 \mathrm{C}$ ) shows this effect together with areas where no Bodipy TMR-PIP ${ }_{2}$ fluorescence is found. The apparent coarsening might be attributed to the formation of areas without Bodipy TMR-PIP ${ }_{2}$ molecules, which are too small to be resolved by means of CLSM. 


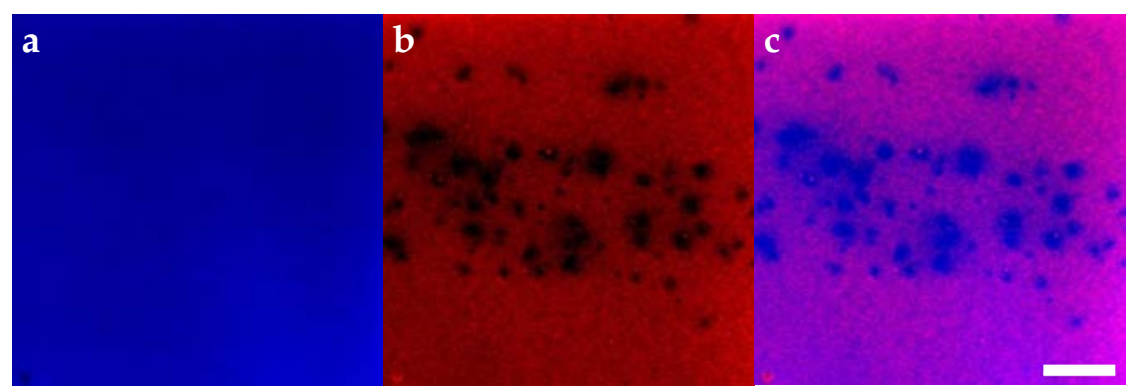

Figure 4.13: Confocal fluorescent image of SHM after incubation with $\mathrm{Ca}^{2+}$ (50 mM). (a) blue channel: Perylene, (b) red channel: Bodipy TMR-PIP ${ }_{2}$ (c) overlay. Scale bar: $10 \mu \mathrm{m}$.

Similar to the experiments with $\mathrm{Ca}^{2+}$, we investigated the influence of ezrin binding on the distribution of Bodipy TMR-PIP $\mathrm{P}_{2}$ within the membrane. After a $2 \mathrm{~h}$ incubation period with $0.5 \mathrm{\mu m}$ AlexaFluor488 labeled ezrin and subsequent rinsing with E1 buffer, the Perlyene fluorescence was still homogeneous, whereas Bodipy TMR-PIP ${ }_{2}$ colocalized with the AlexaFluor 488 labeled ezrin showing the specificity of the ezrin-PIP ${ }_{2}$-interaction (figure 4.14).

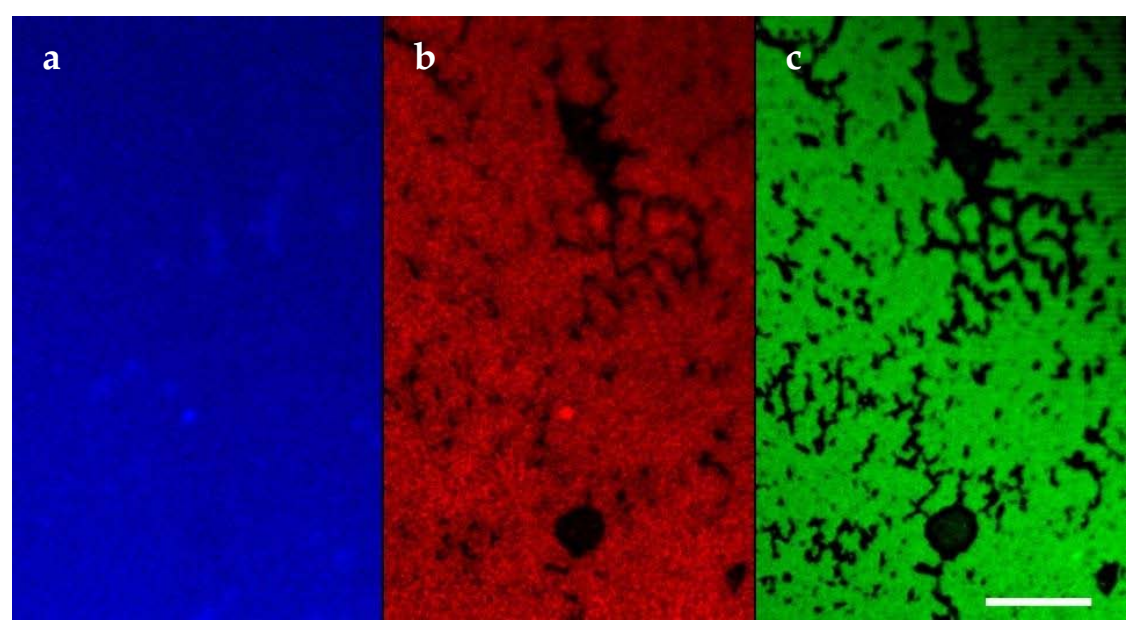

Figure 4.14: Confocal fluorescent image of SHM after incubation with AlexaFluor488 labeled ezrin. (a) blue channel: Perylene, (b) red channel: Bodipy TMR-PIP ${ }_{2}$ (c) green channel: AlexaFluor488 labeled ezrin. Scale bar: $10 \mu \mathrm{m}$.

The black areas in the Bodipy TMR-PIP ${ }_{2}$ and AlexaFluor488 channel are assigned to areas without the respective fluorophor. These areas, however, are rather irregularly shaped as compared to the ones formed upon $\mathrm{Ca}^{2+}$ addition. The exclusion of Bodipy TMR-PIP 2 fluorophores is not uniform. On the one hand, there are areas as depicted in figure 4.14, but on the other hand, a rather homogeneous distribution of Bodipy TMR-PIP 2 and AlexaFluor488 labeled ezrin can be observed. The deviations in the $\mathrm{PIP}_{2}$ distribution due to ezrin binding might be caused by local differences in ezrin concentration. 


\section{$4 \cdot 3$ DISCUSSION}

\subsubsection{Influence of spreading conditions on solid-supported lipid bilayers ( 8 mol\% PIP $\left.{ }_{2}\right)$}

One membrane, different thicknesses

The formation of a lipid bilayer was successful for all spreading protocols, indicated by a characteristic increase in optical thickness in RIfS experiments. The Perylene fluorescence was found to be homogeneous in case of spreading procedures $B$ and C, whereas protocol A yielded membranes exhibiting different levels of Perylene intensity. Deviations of spreading procedure A from B and C were also observed with respect to the recorded $\triangle O T$ values upon vesicle spreading.The increase was with $\triangle O T=10.2 \mathrm{~nm}$ significantly higher than those obtained for the protocols $\mathrm{B}$ and $C$, which amount to $6.4 \mathrm{~nm}$ and $6.3 \mathrm{~nm}$, respectively. These values rather correspond to the typical changes in optical thickness due to bilayer formation $(\triangle O T=$ $6.0 \mathrm{~nm}$ to $6.5 \mathrm{~nm}$ ) determined in RIfS experiments. According to equation 3.19, the change in optical thickness $\triangle O T$ is the product of layer thickness and refractive index. Assuming that the refractive index of a lipid bilayer is $n=1.5,{ }^{[125]}$ a bilayer thickness of $4.4 \mathrm{~nm}$ and $4.2 \mathrm{~nm}$ are calculated for spreading protocol B and C, respectively. This is in the range of $(3.98 \pm 0.08) \mathrm{nm}$ for a POPC bilayer, determined via simultaneous analysis of small angle neutron and X-ray scattering data. ${ }^{[126]}$ The slightly higher bilayer thickness in our case is most probable due to the incorporation of $\mathrm{PIP}_{2}$, whose head group is assumed to protrude from the lipid bilayer up to $(5.99 \pm 1.26) \AA$ with respect to the bridging phosphate diester. ${ }^{[87]}$ However, the apparent high optical thickness in case of spreading protocol A, resulting in bilayer thickness of $6.8 \mathrm{~nm}$, can most likely be attributed to excess vesicles adsorbed to the lipid bilayer. Although the amount of vesicles was reduced by rinsing with EDTAcontaining buffer, no complete detachment was achieved, resulting in an apparent bilayer thickness of $5.5 \mathrm{~nm}$.

One membrane, different $\mathrm{PIP}_{2}$ accessibility

The influence of the different spreading conditions on the $\mathrm{PIP}_{2}$ accessibility in terms of ezrin binding was remarkable. It should be noted that all membranes were treated after spreading such that identical conditions for protein adsorption were ensured. Although all spreading procedures led to membrane formation, only SLBs prepared according to protocol $\mathrm{C}$ showed significant protein coverage. An increase in optical thickness of $\triangle O T=4.7 \mathrm{~nm}$ arising from ezrin binding could be observed 
(figure $4.7 \mathrm{C}_{2}$, RIfS). If the refractive index of an adsorbed protein layer is assumed to be $n=1.5,{ }^{[127]}$ the layer thickness amounts to $3.1 \mathrm{~nm}$ according to equation 3.19. This is in good agreement with the height of ezrin on $\mathrm{PIP}_{2}$-containing SLBs, obtained by atomic force imaging (chapter 5). In addition, CLSM imaging revealed complete surface coverage due to ezrin binding, as can be inferred from the homogeneously distributed AlexaFluor488 labeled ezrin on the $\mathrm{PIP}_{2}$-containing membrane (figure $4.7 \mathrm{C} 2, \mathrm{CLSM}$ ). This is in accordance with quartz-crystal microbalance (QCM) experiments showing that a $\mathrm{PIP}_{2}$ content of $\sim 7 \mathrm{~mol} \%$ is sufficient to achieve maximum protein coverage of solid-supported membranes. ${ }^{[14]}$

Only minor ezrin binding, indicated by $\triangle O T=0.6 \mathrm{~nm}$, was observed for procedure $\mathrm{B}$, yielding a layer thickness of $0.4 \mathrm{~nm}$ according to equation 3.19. This value, however, is substantially lower than the one determined in case of spreading protocol C. This apparently low height of the protein layer can be rationalized by the fact that the recorded RIfS signal is averaged over a region of $1 \mathrm{~mm}^{2}$. Consequently, the height directly relies on the degree of protein coverage. As indicated by CLSM, no complete ezrin surface coverage was observed. Instead, protein clusters in the size of several micrometers were formed besides smaller clusters.

In case of spreading protocol $\mathrm{A}$, only spotlike ezrin adsorption to the $\mathrm{PIP}_{2}$ containing membrane was observed as indicated by the bright green AlexaFluor 488 fluorescence, while no ezrin binding was detectable in the RIfS experiment (figure 4.4 A2, RIfS). This difference can be attributed to data acquisition in RIfS as well since the effective adsorption averaged over the detection area is too small to be detected.

Obviously, both protocol A and B appear to change the accessibility of the $\mathrm{PIP}_{2}$ head group as compared to procedure C. As we employ identical conditions for protein binding, the differences among the spreading protocols most probably originate owing to changed $\mathrm{PIP}_{2}$ distributions within the membrane. With regard to an inhomogeneous $\mathrm{PIP}_{2}$ distribution, there are two possibilities: (i) the spreading conditions induce $\mathrm{PIP}_{2}$ cluster formation on both sides of the membrane and (ii) an asymmetric distribution across the leaflets is obtained, i.e., $\mathrm{PIP}_{2}$ is predominantly located in the proximal leaflet of the SLB and thus not available for ezrin binding. In the following, we will first consider the effect of $\mathrm{Ca}^{2+}$ and then the influence of $\mathrm{Na}^{+}$on $\mathrm{PIP}_{2}$.

Wang et al. could show in both fluorescence and AFM studies that $\mathrm{PIP}_{2}$-containing monolayers $(50 \mathrm{~mol} \%)$ transferred to glass substrates after addition of $1 \mathrm{~mm} \mathrm{Ca}{ }^{2+}$ were characterized by inhomogeneous $\mathrm{PIP}_{2}$ distribution due to phase demixing. In AFM and TEM (transmission electron microscopy) experiments with $1 \mathrm{~mol} \% \mathrm{PIP}_{2}$ in presence of $1 \mu \mathrm{M} \mathrm{Ca}^{2+}$, they determined the cluster size to $(40 \pm 11) \mathrm{nm} .{ }^{[128]} \mathrm{Lev}-$ ental et al. demonstrated that the lateral organization of $\mathrm{PIP}_{2}$ is sensitive to small 
changes in $\mathrm{Ca}^{2+}$ concentration. In combined Langmuir film balance and epifluorescence experiments, they could show that $\mathrm{PIP}_{2}$-rich domains were formed upon addition of $\mathrm{Ca}^{2+}$. By modeling the change in surface pressure as a function of the $\mathrm{Ca}^{2+}$ concentration by first-order reversible binding with a $\mathrm{pH}$ value independent dissociation constant, they determined a $K_{\mathrm{d}}$ value of $\sim 3 \mu \mathrm{M} .{ }^{[129]}$ The clustering effect of $\mathrm{Ca}^{2+}$, however, is not restricted to monolayers, as phase demixing within giant unilamellar vesicles was observed upon $\mathrm{Ca}^{2+}$ addition. ${ }^{[101,128]}$ Therefore, we expected to induce $\mathrm{PIP}_{2}$ clustering upon $\mathrm{Ca}^{2+}$ addition in our measurements as well. However, all studies mentioned above could show that the clustering effect was reversible by adding corresponding concentrations of EDTA or EGTA, respectively. For this reason, we believed to reverse $\mathrm{Ca}^{2+}$ induced $\mathrm{PIP}_{2}$ clustering by rinsing with EGTA-containing buffer. However, the presence of $\mathrm{Ca}^{2+}$ during membrane formation appeared to hamper the binding of ezrin to $\mathrm{PIP}_{2}$, although EGTA should have removed $\mathrm{Ca}^{2+}$ from the system. This led to the question whether EGTA chelates $\mathrm{Ca}^{2+}$ efficiently or whether there were still amounts of $\mathrm{Ca}^{2+}$ present. The apparent dissociation constant of EGTA and $\mathrm{Ca}^{2+}$ at $\mathrm{pH} 7.1$ amounts to $K_{\mathrm{d}}=131 \mathrm{nM}^{[130]}$, which is significantly lower than the $K_{d}$ value for $\mathrm{Ca}^{2+}$ and $\mathrm{PIP}_{2}$ determined by Levental et al. $(\sim 3 \mu \mathrm{M}) .{ }^{[129]}$ Hence, $\mathrm{Ca}^{2+}$ should be chelated by appropriate EGTA concentration and consequently be removed from the solution. However, it might be that the aggregation of $\mathrm{PIP}_{2}$ within the membrane is maintained because of hydrogen bonding between individual $\mathrm{PIP}_{2}$ molecules. ${ }^{\left[{ }^{131-133]}\right.}$ We will discuss this aspect in more detail in the following.

$\mathrm{Ca}^{2+}$ induced asymmetric $\mathrm{PIP}_{2}$ distribution across the leaflets has not been reported to the best of our knowledge in SLBs so far. Rossetti et al. demonstrated by evaluating the mobility of labeled phosphatidylserine (PS), an anionic phospholipid, that most of the PS within a phosphatidylcholine matrix on $\mathrm{TiO}_{2}$ is facing the oxide surface in presence of $\mathrm{Ca}^{2+}$, suggesting a bridging effect. ${ }^{\left[{ }^{134}\right]}$ However, they showed that this behavior is surface specific as no asymmetric PS distribution was observed within SLBs prepared on $\mathrm{SiO}_{2}$. If we dealt with an asymmetric transbilayer distribution of $\mathrm{PIP}_{2}$ in terms of $\mathrm{PIP}_{2}$ enrichment in the proximal leaflet, we would expect an immobile fraction $>50 \%$ of PIP 2 in FRAP experiments. We were not able to substantiate this idea in experiments. Accordingly, it appears that an asymmetric $\mathrm{PIP}_{2}$ distribution across the leaflets caused by $\mathrm{Ca}^{2+}$ bridging is rather unlikely.

In contrast to $\mathrm{Ca}^{2+}, \mathrm{Na}^{+}$is monovalent and thus not able to bridge $\mathrm{PIP}_{2}$. Contrary effects of $\mathrm{Ca}^{2+}$ and $\mathrm{Na}^{+}$on the surface pressure of pure $\mathrm{PIP}_{2}$ monolayers were reported by Levental et al. ${ }^{[133]} \mathrm{Ca}^{2+}$ caused a drop in surface pressure, thus indicating the bridging of $\mathrm{PIP}_{2}$ within the monolayer, whereas $\mathrm{Na}^{+}$exhibited an expanding effect, i.e, an increased surface pressure was detected upon $\mathrm{Na}^{+}$addition. 
Interestingly, a purely electrostatic mechanism could be excluded as monolayers containing other anionic lipids such as phosphatidylserine were not affected in the same way. Instead, a slight contraction of the monolayer was observed upon increased ionic strength owing to reduced electrostatic repulsion. The authors argued that $\mathrm{Na}^{+}$disrupts the hydrogen-bonded network of $\mathrm{PIP}_{2}$. Accordingly, one would assume a rather homogeneous distribution of $\mathrm{PIP}_{2}$ within the membrane in presence of $\mathrm{Na}^{+}$. However, we used a significantly lower $\mathrm{PIP}_{2}$ content of $8 \mathrm{~mol} \%$ and bilayers instead of monolayers. For this reason, a direct correlation might be only valid to a limited extent.

Nevertheless, several lines of evidence suggest significant contribution of hydrogen bonding with regard to local accumulation of $\mathrm{PIP}_{2}$. Redfern et al. investigated the domain forming tendency of $\mathrm{PIP}_{2}$ within phosphatidylcholine vesicles employing different biophysical techniques. ${ }^{\left[{ }^{132]}\right.}$ The authors reported a $\mathrm{pH}$-dependent formation of $\mathrm{PIP}_{2}$ enriched domains, which they attributed to hydrogen bond mediated attraction. Enhanced domain formation was observed for $\mathrm{pH}$ values $>\mathrm{pK}_{\mathrm{a} 2}$, indicating that the deprotonated $4^{\prime}$ and $5^{\prime}$-phosphomonoester groups act as hydrogen bond acceptors. In the first place, this is rather surprising as one might expect increased repulsive forces because of deprotonation. However, it appears that attractive forces including hydroxyl/phosphomonoester, hydroxyl/phosphodiester, and hydroxyl/hydroxyl interactions overcome electrostatic repulsion. Notably, the loss of proper hydrogen bond acceptors as a consequence of lowering the $\mathrm{pH}$ value leads to enhanced lipid mixing. Liepina et al. corroborated the hydrogen bond induced $\mathrm{PIP}_{2}$ aggregation in dynamic molecular simulations showing that $\mathrm{PIP}_{2}$ clusters within a phosphatidylcholine lipid bilayer are stabilized via hydrogen bonding. ${ }^{\left[{ }^{131}\right]}$ It appears that the $\mathrm{PIP}_{2}$ distribution is regulated by a subtle interplay between hydrogen bonding and electrostatic repulsion. Hydrogen bonding should favor the segregation of $\mathrm{PIP}_{2}$, whereas repulsive forces should produce homogeneously distributed $\mathrm{PIP}_{2}$.

Obviously, further evaluation concerning the distribution of $\mathrm{PIP}_{2}$ as a function of applied spreading conditions is necessary. For this purpose, the fluorescent $\mathrm{PIP}_{2}$ analogue Bodipy TMR-PIP 2 was incorporated into the membrane to visualize the distribution of PIP ${ }_{2}$. SLBs were prepared by spreading SUVs composed of POPC/ $\mathrm{PIP}_{2}$ /Bodipy TMR-PIP 2 /Perylene (91:7.5:0.5:1) on silicon substrates according to the respective spreading protocol. Notably, differences with respect to the $\mathrm{PIP}_{2}$ distribution within the membrane were detected by CLSM (figure 4.15). SLBs prepared according to protocol A were characterized by excess vesicles adsorbed to the membrane surface as already observed in figure 4.4 A1 CLSM and 4.5. The number of adhered vesicles could be reduced to a certain degree by rinsing with AE buffer. However, it was impossible to achieve complete removal as indicated 
in figure 4.15 by the bright fluorescent structures. The application of spreading protocol B lead to rather inhomogeneously dispersed Bodipy TMR-PIP ${ }_{2}$, whereas spreading protocol C yielded a comparably homogeneous distribution of the $\mathrm{PIP}_{2}$ analogue.

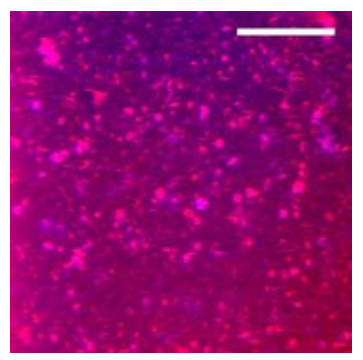

Protocol A

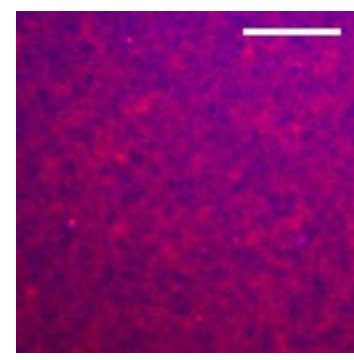

Protocol B

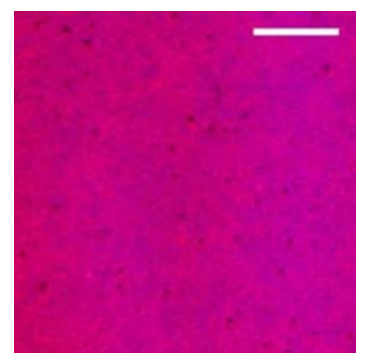

Protocol C

Figure 4.15: Confocal fluorescent images illustrating the influence of spreading conditions on $\mathrm{PIP}_{2}$ distribution. Overlay of blue Perylene fluorescence and red Bodipy TMR-PIP fluorescence to visualize the distribution of $\mathrm{PIP}_{2}$. Lipid composition: $\mathrm{POPC} / \mathrm{PIP}_{2} /$ Bodipy TMR-PIP $_{2} /$ Perylene (91:7.5:0.5:1). Scale bars: $10 \mu \mathrm{m}$.

A distinct influence of spreading conditions on the $\mathrm{PIP}_{2}$ distribution emerges. In light of these experiments, the deviation in protein binding as a function of spreading might be based on different $\mathrm{PIP}_{2}$ distributions.

In case of spreading protocol $\mathrm{A}$, the $\mathrm{Ca}^{2+}$ induced clustering of $\mathrm{PIP}_{2}$ was not reversed by rinsing with $\mathrm{Ca}^{2+}$-chelating buffer, preventing full protein surface coverage. Moreover, the tightly adhering excess vesicles impair any application of these SLBs in connection with surface-sensitive techniques.

In case of spreading protocol $\mathrm{B}$, the rather inhomogeneous distribution of $\mathrm{PIP}_{2}$ was not expected as such. Assuming that $\mathrm{Na}^{+}$is able to reduce electrostatic repulsion as well as to disrupt hydrogen bonds, a homogeneous distribution of $\mathrm{PIP}_{2}$ should be preferred. A speculative assumption is that the inhomogeneities might be the result of the subtle interplay of hydrogen bonds and reduced repulsive forces. $\mathrm{Na}^{+}$reduces the electrostatic repulsion, thus allowing PIP 2 molecules to come closer. This might enhance hydrogen bonding if the $\mathrm{Na}^{+}$concentration is not too high and consequently cause the formation of $\mathrm{PIP}_{2}$ enriched domains as indicated in figure 4.15. However, to test this hypothesis further experiments are required investigating the effect of variation in $\mathrm{pH}$, similar to the work of Redfern et al. with $\mathrm{PIP}_{2}$-containing vesicles. ${ }^{[132]}$ Spreading at low $\mathrm{pH}$ values should always produce homogeneously dispersed $\mathrm{PIP}_{2}$ as the number of proper hydrogen bond acceptors is reduced.

Notably, $\mathrm{PIP}_{2}$ appears to be rather homogeneously dispersed upon vesicle spreading according to protocol C (figure 4.15). Lowering the $\mathrm{pH}$ value decreases the net charge of $\mathrm{PIP}_{2}$, thus reducing the electrostatic repulsion among $\mathrm{PIP}_{2}$ molecules. Moreover, it decreases the number of proper hydrogen bond acceptors via protona- 
tion and accordingly the ability of $\mathrm{PIP}_{2}$ to form hydrogen bonds between individual $\mathrm{PIP}_{2}$ molecules.

In conclusion, we propose that the apparent reduced ezrin adsorption in case of spreading protocol A might be attributed to hydrogen bonds maintaining the $\mathrm{Ca}^{2+}$ induced PIP 2 clusters. Accordingly, it would be impossible to achieve complete protein surface coverage as $\mathrm{PIP}_{2}$ is not homogeneously distributed within the membrane. A similar explanation might account for the obvious $\mathrm{PIP}_{2}$ inhomogeneities in case of spreading protocol B. However, it remains elusive why no $\mathrm{PIP}_{2}$ redistribution is observed when the spreading buffers are exchanged for the protein buffer E1, which is considerably lower in ionic strength and contains no $\mathrm{Ca}^{2+}$. Considering that the diffusion length of a lipid molecule with a diffusion coefficient of $3 \mu \mathrm{m}^{2} / \mathrm{s}$ over $60 \mathrm{~s}$ amounts to $\sim 27 \mu \mathrm{m}$, we expected that the $\mathrm{PIP}_{2}$ lipids would be randomly redistributed in absence of clustering factors. Whether hydrogen bonding can account solely for the inhomogeneous distribution of $\mathrm{PIP}_{2}$ should be revealed upon the addition of uncharged chaotropes, such as urea, disrupting the aggregation of $\mathrm{PIP}_{2}$. Moreover, esterification of the phosphate groups in $\mathrm{PIP}_{2}$ should give additional information whether hydrogen bonding is causing inhomogeneities in the $\mathrm{PIP}_{2}$ distribution.

\subsubsection{PIP 2 mobility within solid-supported membranes (3 mol\% PIP ${ }_{2}$ )}

High immobile fractions of Bodipy TMR-PIP ${ }_{2}$ in solid-supported lipid bilayers

The lateral mobility of Bodipy TMR-PIP ${ }_{2}$ as $\mathrm{PIP}_{2}$ tracer was investigated within a POPC matrix by means of FRAP. Considering that all experiments were carried out at room temperature, the lateral diffusion coefficients of $\sim 3 \mu \mathrm{m}^{2} / \mathrm{s}$ for both SHMs and SLBs were in good agreement with values for POPC SLBs, determined by deuterium nuclear magnetic resonance (NMR) relaxation. ${ }^{[35]}$ Interestingly, differences with respect to the mobile fractions emerged between SHM and SLB setup. The mobile fraction in case of the SHM setup amounts to almost $100 \%$, whereas it is drastically reduced to $52 \%$ for SLBs. This indicates that in the latter case, a symmetrical transbilayer distribution of $\mathrm{PIP}_{2}$ with hindered diffusion within the proximal leaflet may be the reason for the decrease in mobile fraction to $\sim 50 \%$. This is in accordance with observations made by Bosk. ${ }^{\left[{ }^{123}\right]}$ In general, SLBs should retain their fluidity due to the thin, lubricant water layer between bilayer and substrate. ${ }^{[136]}$ However, Hetzer et. al could show by means of NMR that frictional coupling between substrate and proximal leaflet influences significantly the lateral diffusion of lipids within this monolayer. Surprisingly, the distal leaflet is not affected, thus indicating low friction forces between the two monolayers. ${ }^{[137]}$ These results suggest 
that an averaged, reduced diffusion coefficient may be obtained. However, within the time frame of the experiments the diffusion within the distal leaflet may be neglected. Considering both mobile fraction of $50 \%$ and similar diffusion coefficient to the one in case of SHMs, we suggest that the diffusion in the proximal leaflet is drastically reduced, whereas the diffusion in the distal leaflet remains unhindered, determining the average diffusion coefficient.

Effects of calcium and ezrin: Slowing down PIP ${ }_{2}$

We observed upon the addition of either $\mathrm{Ca}^{2+}$ or ezrin slowed diffusion of the fluorescent $\mathrm{PIP}_{2}$ analogue Bodipy TMR-PIP ${ }_{2}$ within SHMs, thus indicating the formation of $\mathrm{PIP}_{2}$ clusters. The same trend was reported by Wang et al. in fluorescence correlation spectroscopy (FCS) experiments. They found a retardation of the diffusion coefficient of Bodipy TMR-PIP ${ }_{2}$ in GUVs to $(0.8 \pm 0.4) \mu^{2} / \mathrm{s}$ in presence of $10 \mu \mathrm{M} \mathrm{Ca}^{2+} .{ }^{[128]}$ However, this value is even below the diffusion coefficient of $(1.4 \pm 0.4) \mathrm{\mu m}^{2} / \mathrm{s}$ we determined for Bodipy TMR-PIP ${ }_{2}$ within SHMs upon incubation in $\mathrm{Ca}^{2+}$-containing buffer $(50 \mathrm{mM})$ and subsequent rinsing with EDTA-free E1 buffer. As a matter of fact, we should expect to observe a lower diffusion coefficient as we employ model membranes on solid support rather than GUVs and we determined the diffusion coefficients by means of FRAP instead of FCS measurements. ${ }^{[138,139]}$ According to Guo et al., the diffusion coefficient within model membranes is shifted to larger values when measuring on small length scales as in case of FCS measurement, whereas for FRAP experiments, measuring on large length scales should produce smaller values. However, the diffusion coefficient most probably relies on the size of the formed $\mathrm{PIP}_{2}$ cluster as such that larger aggregates should diffuse slower than smaller ones. Assuming that the formation of larger $\mathrm{PIP}_{2}$ clusters is favored within GUVs owing to the higher mobility of lipids as compared to SLBs and the possibility to generate membrane curvature to accommodate the large lipid head group, we might expect smaller $\mathrm{PIP}_{2}$ clusters within SHMs and accordingly an increased diffusion coefficient. This is corroborated by the observation of $\mathrm{PIP}_{2}$ clustering within GUVs in presence of $\mathrm{Ca}^{2+}$ by means of CLSM. ${ }^{[101,128]}$ On the contrary, we observed rather a coarsening of the Bodipy TMR-PIP $_{2}$ fluorescence within SHMs than the formation of distinct PIP $_{2}$ clusters, although some regions depleted in Bodipy TMR-PIP 2 were detected (figure 4.13). Considering that Wang et al. determined the size of $\mathrm{Ca}^{2+}$ induced $\mathrm{PIP}_{2}$ clusters to $(40 \pm 11) \mathrm{nm}$ in solid-supported monolayers (10 $\mathrm{mol}_{\%} \mathrm{PIP}_{2}$ ) by means of AFM and TEM, it appears likely that we are not able to resolve these clusters owing to the limited resolution of the CLSM. ${ }^{[128]}$ 
Golebiewska et al. addressed the question of different $\mathrm{PIP}_{2}$ pools within Rat1 cells by microinjection of Bodipy TMRPIP ${ }_{2}$ and subsequent measurements of its diffusion. ${ }^{\left[{ }^{140]}\right.}$ They determined an average diffusion coefficient for Bodipy TMR$\mathrm{PIP}_{2}$ of $(0.9 \pm 0.2) \mu^{2} / \mathrm{s}$ in the unperturbed inner leaflet of the plasma membrane, while a value of $(2.5 \pm 0.8) \mu^{2} / \mathrm{s}$ was derived for its diffusion in blebs formed on the plasma membrane. Blebs are characterized by a rather poor coupling to the cytoskeleton, thus resembling a typical artificial phospholipid bilayer. Accordingly, the determined values are in rather good agreement with our diffusion coefficients of Bodipy TMR-PIP ${ }_{2}$ within SLBs or SHMs, $(2.9 \pm 0.3) \mu^{2} / \mathrm{s}$ and $(3.0 \pm 0.5) \mu \mathrm{m}^{2} / \mathrm{s}$, respectively. An average diffusion coefficient of $(3.3 \pm 0.8) \mu \mathrm{m}^{2} / \mathrm{s}$ for Bodipy TMR$\mathrm{PIP}_{2}$ in GUVs was found in a previous study, indicating the slightly lower lateral mobility of lipids in SSMs as compared to vesicle membranes lacking the solid support. ${ }^{[141]}$ Notably, the addition of the cytoskeletal linker protein ezrin decreased the lateral mobility of Bodipy TMR-PIP ${ }_{2}$ to $(0.7 \pm 0.5) \mu \mathrm{m}^{2} / \mathrm{s}$, similar to the value Golebiewska et al. found in the unperturbed inner leaflet of the plasma membrane. ${ }^{\left[{ }^{140]}\right.}$ We conclude that ezrin is able to slow down the diffusion of $\mathrm{PIP}_{2}$ within artificial membranes at complete surface coverage of ezrin. Moreover, we observed the formation of domains enriched in Bodipy TMR-PIP ${ }_{2}$, which colocalized with ezrin covered areas indicating that ezrin may be able to induce clustering of PIP $_{2}$. The question whether ezrin binding leads to reorganization of $\mathrm{PIP}_{2}$ is still a matter of controversy. Based on self-quenching assay with fluorescent analogues of $\mathrm{PIP}_{2}$, Blin et al. argued that ezrin does not induce the clustering of $\mathrm{PIP}_{2}$, whereas MARCKS does. ${ }^{[119]}$ However, it might be a function of the local ezrin concentration as the same group suggested reorganization of PIP $\mathrm{P}_{2}$ by ezrin in GUVs using ezrin concentrations up to $\sim 15 \mu \mathrm{M} .{ }^{[101]}$ In this study, they reported the colocalization of $\mathrm{PIP}_{2}$ fluorophores and fluorescently labeled ezrin at GUVs and apparently clustered ezrin-PIP ${ }_{2}$ complexes in some places, indicated by enhanced fluorescence. Although we employed lower concentrations of ezrin, this is in good agreement with our observations. We always found colocalization of ezrin and $\mathrm{PIP}_{2}$. Mostly, the distribution of $\mathrm{PIP}_{2}$ remained apparently homogeneous upon ezrin addition. However, in some places we observed regions depleted in $\mathrm{PIP}_{2}$ and ezrin, thus suggesting that ezrin might be able to reorganize $\mathrm{PIP}_{2}$. Accordingly, further reduction of the $\mathrm{PIP}_{2}$ content may clarify the reorganization capabilities of ezrin. This process might depend on the lateral protein-protein interaction, which were observed by AFM imaging of ezrin on SLBs. ${ }^{[117]}$ The authors proposed that the ezrin monolayer is formed emanating from few nucleation sites by attractive lateral protein-protein interactions on the membrane. 


\subsection{CONCLUSION}

In this work, we presented three strategies that allowed the reliable preparation of SLBs on silicon support by spreading SUVs containing up to $8 \mathrm{~mol} \% \mathrm{PIP}_{2}$. We demonstrated that a thorough investigation of the obtained membranes is pivotal to evaluate the influence of different spreading conditions on membrane formation and subsequent protein binding. The spreading protocols employing either $2 \mathrm{mM} \mathrm{Ca}^{2+}, 200 \mathrm{mM} \mathrm{Na}^{+}$, or $\mathrm{pH} 4.8$, were evaluated by means of RIfS and CLSM with respect to binding of ezrin, a cytoskeleton-membrane linker protein, which binds specifically to $\mathrm{PIP}_{2}$. We were able to show that the agents employed during the spreading process have a considerably strong influence on ezrin binding. We suggest that this behavior is attributed to different PIP $_{2}$ distributions caused by the influence of spreading agents. In particular, we strongly advise to refrain from using $\mathrm{Ca}^{2+}$ as spreading additive in connection with $\mathrm{PIP}_{2}$ containing vesicles. SLBs prepared in presence of $\mathrm{Ca}^{2+}$ were characterized by a high number of adhered excess vesicles, which was not significantly reduced even after rinsing with $\mathrm{Ca}^{2+}$-chelating buffer. Consequently, such membranes are not suitable for the application of surface sensitive techniques. Increasing the ionic strength produced unexpectedly a rather inhomogeneous distribution of $\mathrm{PIP}_{2}$, which is obstructive if e.g. the $\mathrm{PIP}_{2}$ sequestration properties of a protein are to be investigated. However, we found suitable spreading conditions by lowering the $\mathrm{pH}$ value to $4.8 \mathrm{pH}$ during the spreading process. This protocol allowed both the reliable membrane formation even in presence of high molar fractions of $\mathrm{PIP}_{2}$ and the subsequent binding of ezrin. It is worth to mention that SLBs containing $<4 \mathrm{~mol} \% \mathrm{PIP}_{2}$ can also be prepared at $\mathrm{pH} 7.4$ and similar ezrin coverage is observed in both cases.

Moreover, we presented an alternative approach to prepare solid-supported membranes with high $\mathrm{PIP}_{2}$ content. SHMs were formed upon spreading of $\mathrm{PIP}_{2}$ containing vesicles on silanized silicon substrates. This setup is in particular useful for the determination of diffusion coefficients and immobile fractions by means of FRAP as the fluorescence intensity contribution is reduced to the distal leaflet. Accordingly, the lack of the often rather immobile proximal leaflet in SLBs does not hamper the FRAP data analysis in case of SHMs. The fluorescently labeled $\mathrm{PIP}_{2}$ analogue Bodipy TMR-PIP ${ }_{2}$ was used to evaluate the distribution of $\mathrm{PIP}_{2}$ and its lateral mobility. We were able to show that both $\mathrm{Ca}^{2+}$ and ezrin lead to reduced lateral mobility of Bodipy TMR-PIP ${ }_{2}$ within the membrane and partial PIP 2 reorganization. However, it is not clear whether the $\mathrm{Ca}^{2+}$ dependent sequestration of $\mathrm{PIP}_{2}$ occurs as well in the much more complex cell membrane. Notably, the lateral mobility of $\mathrm{PIP}_{2}$ was reduced upon ezrin binding to a value similar to diffusion coefficients found in cellular plasma membrane. ${ }^{[140]}$ Although it remains elusive whether ezrin promotes 
$\mathrm{PIP}_{2}$ clustering within the cell, it appears reasonable that it might at least be able to maintain preformed aggregates.

\section{$4 \cdot 5$ CO-WORKERS}

Kramer, Corinna (RIfS and CLSM experiments shown in figures 4.4, 4.6, 4.7); Morick, Daniela (CLSM experiments shown in figures 4.4, 4.6, 4.7)

\subsection{EXPERIMENTAL DETAils}

MATERIALS The lipids L- $\alpha$-phosphatidylinositol-4,5-bisphosphate (PIP ${ }_{2}$, purified from porcine brain with a fatty acid composition primarily composed of 18:0, 18:1, and 20:4 acyl chains) and 1-palmitoyl-2-oleoyl-sn-glycero-3-phosphocholine (POPC) were obtained from Avanti polar lipids (Alabaster, USA). The fluorescently labeled $\mathrm{PIP}_{2}$ analogue Bodipy TMR-PIP ${ }_{2}\left(\mathrm{C}_{1} 6\right)$ was from Echelon Biosciences (Salt Lake City, USA). The fluorophores Perylene and AlexaFluor488 $\mathrm{C}_{5}$-maleimide were obtained from Life Technologies (Darmstadt, Germany). Dodecyl-trichlorosilane (DTS) was from Sigma-Aldrich (Steinheim, Germany). Silicon wafers were purchased from Silicon materials (Kaufering, Germany). All chemicals were of the highest purity available. Water was purified first through a Millipore water purification system Milli-RO 3 plus and finally with a Millipore ultrapure water system Milli-Q plus 185 (specific resistance $=18.2 \mathrm{M} \Omega / \mathrm{cm}$ ) (Billerica, USA).

EXPERIMENTAL Details on the preparation of lipid films, SUVs and SLBs are listed in section 3.2.2. The silanization and subsequent formation of SHBs is explained in section 3.2.1.2 and 3.2.2.3.

The experimental procedure of RIfS and SPR experiments can be found in section 3.3.3. Details on FRAP experiments as well as a detailed description concerning the data analysis is listed in section 3.3.2.1. 
THE ACTIVATION PROCESS OF EZRIN: ON THE TRAIL OF THE CONFORMATIONAL CHANGE

\subsection{INTRODUCTION}

Ezrin, radixin, and moesin: A family of membrane-cytoskeleton linkers

A finely tuned linkage of the plasma membrane and the subjacent actin cytoskeleton is a prerequisite for the organization and dynamic characteristics of the cell membrane architecture. Members of the ezrin-radixin-moesin (ERM) protein family are known to provide such a regulated connection, thus participating in adhesion, motility, and fundamental developmental processes. ${ }^{[1,3]}$ Ezrin was identified as $80 \mathrm{kDa}$ component of the microvillar cytoskeleton in 1983 and named in recognition of Ezra Cornell University. ${ }^{[142]}$ In 1988, Moesin (membrane-organizing extension spike protein) was isolated from bovine uteri and identified as receptor protein for heparin. ${ }^{[143]}$ Radixin was characterized as an actin-binding protein and found to be highly concentrated in the undercoat of adherens junctions of rat liver cells in 1989. ${ }^{\left[{ }^{[44]}\right.}$ ERM proteins have been examined in culture cell lines and various tissues with respect to their localization. Even though they are coexpressed in most cultured cell lines, ${ }^{\left[145^{-147]}\right.}$ cells within the body show different and even restricted patterns of expression. Epithelial and mesothelial cells express ezrin, whereas moesin is expressed in the endothelium. ${ }^{[148]}$ In microvilli of epithelial cells only ezrin was found, while in hepatocytes exclusively radixin is present. ${ }^{[149]}$ Notably, moesin-deficient mice did not develop any distinguishable phenotype, leading to the assumption of redundancy among ERM proteins. ${ }^{[2]}$ However, specific roles for individual ERM proteins have been reported. For instance, radixin-knockout mice show loss of hearing and selective degeneration of cochlear stereocilia, where ezrin substituted for radixin, in the inner ear, thus not being able to compensate for radixin deficiency. ${ }^{[150]}$ At the subcellular level, all ERM proteins are concentrated in actin-rich membrane projections such as microvilli, filopodia, and membrane ruffles. ${ }^{[151]}$ 


\section{Structural homology of ERM proteins}

The ERM proteins exhibit a high degree of $\sim 85 \%$ sequence homology. Consequently, they are characterized by similar structural features (figure 5.1). ${ }^{[1]}$ ERM proteins possess a $\sim 300$ residue $N$-terminal ERM association domain (N-ERMAD), providing membrane attachment via specific interaction with the phosphoinositide L- $\alpha$-phosphatidylinositol-4,5-bisphosphate $\left(\mathrm{PIP}_{2}\right) \cdot{ }^{[10]} \mathrm{N}$-ERMAD is also referred to as FERM (four-point-one, ezrin, radixin, moesin) domain, reflecting the identification of this domain within a protein family of membrane-cytoskeleton linkers. ${ }^{\text {[152] }}$ The C-terminal ERM association domain (C-ERMAD) contains a binding site for filamentous actin (F-actin) mapped to the last 34 amino acids, which is highly conserved among ERM proteins. ${ }^{[6]} \mathrm{N}-E R M A D$ and C-ERMAD are connected via an $\alpha$-helical linker region. Moreover, ezrin and radixin contain a polyproline stretch between $\alpha$-helical and $C$-terminal region. A conserved threonine residue $-\mathrm{T}_{5} 67$ (ezrin), T564 (radixin), and T558 (moesin), respectively - is located within the CERMAD, which is considered to play a pivotal role in the activation of ERM proteins (see below). ${ }^{[3]}$

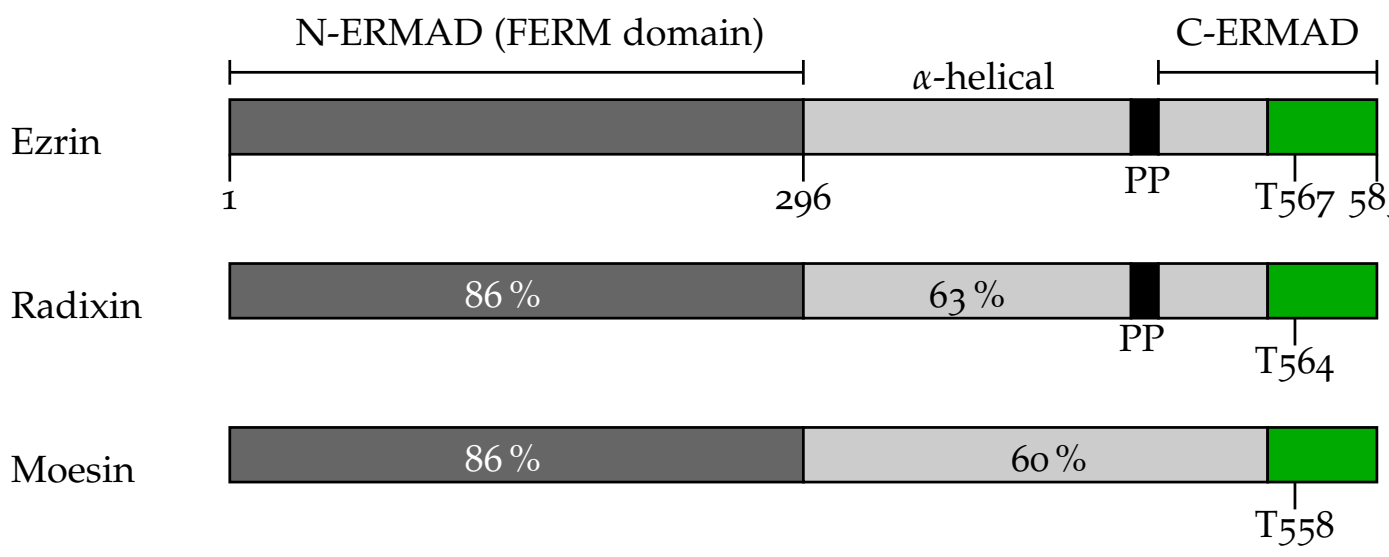

Figure 5.1: Schematic drawing showing the sequence identity of moesin and radixin to ezrin. All ERM proteins are characterized by the $\sim 300$ residue N-ERMAD, also referred to as FERM domain (dark grey). A F-actin binding site (green) is located within the CERMAD containing a conserved threonine residue. Its phosphorylation is involved in the activation of ERM proteins. N-ERMAD and C-ERMAD are connected via an $\alpha$-helical linker region (light gray) with high propensity for coiled-coil formation. Ezrin and radixin contain a polyproline region (black, PP). Adapted from Bretscher et al. [3]

Crystal structures of the $N$-terminal domains of the ERM proteins as well as the moesin FERM/tail complex led to a deeper understanding of the structural arrangement. ${ }^{\left[{ }^{153-155]}\right.}$ The FERM domain forms a clover-shaped structure subdivided into three structural modules $\mathrm{F}_{1}-\mathrm{F}_{3}$ (figure $5.2 \mathrm{~A}$ ). In particular, the $\mathrm{IP}_{3} /$ radixin-FERM complex revealed a possible $\mathrm{PIP}_{2}$ binding site located in the basic cleft between $\mathrm{F}_{1}$ and $\mathrm{F}_{3}$ subdomain. Li et al. succeeded in crystallizing full-length moesin, isolated from Spodoptera frugiperda cells (figure $5.2 \mathrm{~B}$ ). ${ }^{\left[{ }^{156]}\right.}$ It is characterized by $\sim 60 \%$ cDNA 
(complementary deoxyribonucleic acid) sequence identity with human ERM proteins. The structure exhibits typical ERM protein features including the $N$-terminal three-lobed FERM domain, the $\alpha$-helical linker region, and the C-ERMAD. The $\alpha$ helical domain is composed of three extended helices, from which two of them form an antiparallel coiled-coil of $\sim 7 \mathrm{~nm}$ in length. Notably, parts of the $\alpha$-helical linker region appear to block the PIP $_{2}$ binding site located in the basic cleft. This region was identified as inhibitory linker, which is believed to be released after initial association of $\mathrm{PIP}_{2}$ to a binding site located in the $\mathrm{F}_{3}$ subdomain of moesin. ${ }^{\text {[120] }}$ The authors suggested sequential binding of $\mathrm{PIP}_{2}$ to this binding site followed by binding to the one situated within the basic cleft.

A

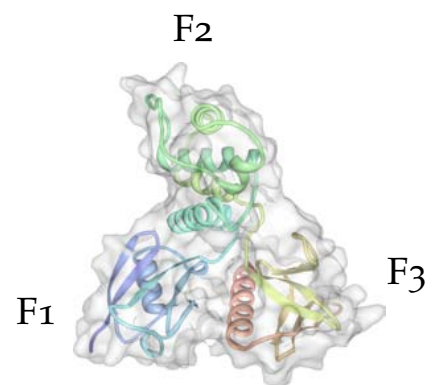

B

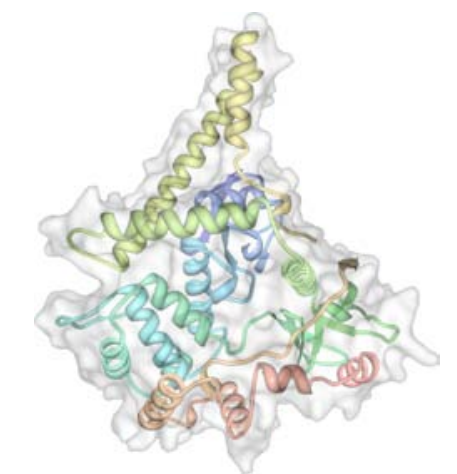

Figure 5.2: A Crystal structure of the FERM domain of ezrin subdivided into three structural modules F1-F3 (PDB ID: 1NI2). B Crystal structure of full-length moesin isolated from Spodoptera frugiperda cells (PDB ID: 2IIJ).

The activation process of ERM proteins

Several lines of evidence indicated that the function of ERM proteins is conformationally regulated by strong interaction between N-ERMAD and C-ERMAD. Based on the expression of either full-length ezrin or its $\mathrm{N}$ - and $\mathrm{C}$-terminal parts in cells, Algrain et al. proposed that ezrin interacts with the plasma membrane via $\mathrm{N}$ ERMAD, while the linkage to the actin cytoskeleton is mediated by C-ERMAD. ${ }^{[157]}$ The relevance of C-ERMAD with regard to association to the actin cytoskeleton was corroborated by Turunen et al. identifying a $\sim 34$ amino acid F-actin binding site in the C-ERMAD of ezrin ${ }^{[6]}$. Gary et al. could show in biochemical studies that self-association of N-ERMAD and C-ERMAD prevented F-actin binding. ${ }^{[7]}$ Owing to the high degree of structural homology, it can be assumed that ERM proteins are characterized by a similar activation mechanism. In this context, a conformational mask model was established proposing the existence of different ERM pools - an inactive, also referred to as dormant state, and an active form exposing the F-actin 
binding site (figure 5.3).
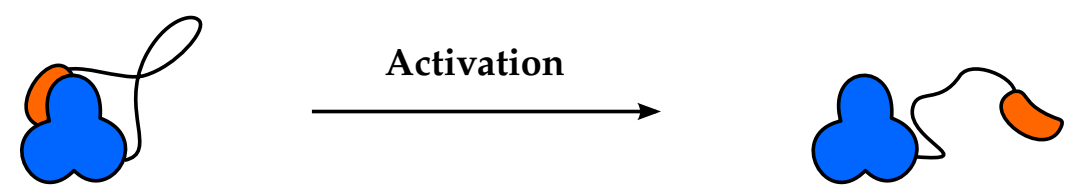

Figure 5.3: Schematic drawing showing conformational regulation of ERM proteins. Selfassociation of N-ERMAD (blue) and C-ERMAD (orange) results in the inactive or dormant conformation. Dissociation is induced by activation leading to the active state.

Consequently, exposure of the F-actin binding site requires the dissociation of the N-ERMAD/C-ERMAD complex induced by activation factors. A threonine (T) residue within the F-actin binding site was identified as strong candidate for this role. This conserved residue was recognized for ezrin, radixin, and moesin $-\mathrm{T}_{5} 67$, $\mathrm{T}_{564}$, and $\mathrm{T}_{558}$, respectively - as target for phosphorylation by Rho-kinase and protein kinase $C \Theta .{ }^{[8,9]}$ Matsui et al. could show that the phosphorylation of this specific threonine by Rho kinase in vitro reduces the N-ERMAD/C-ERMAD interaction, whereas the F-actin binding capability of C-ERMAD is not affected. ${ }^{[9]}$ Furthermore, $\mathrm{PIP}_{2}$ was suggested as candidate for the activation of ERM protein. ${ }^{[158]}$ Several biochemical studies and cellular assays confirmed that $\mathrm{PIP}_{2}$ is not only responsible for membrane attachment but also plays a functional role in the activation process of ERM proteins. ${ }^{[11,159,160]}$ Based on these findings, a two-step model was proposed for ERM activation comprising recruitment to the membrane by $\mathrm{PIP}_{2}$ and subsequent phosphorylation of the conserved threonine residue in the C-ERMAD. ${ }^{[12,13]}$

Several studies mapped the conformational change either by structural changes in the protein or indirectly by monitoring its binding capability to F-actin. Maniti et al. investigated ezrin and moesin with regard to potential secondary structure modifications due to $\mathrm{PIP}_{2}$ binding. The authors could observe that both proteins undergo conformational changes upon binding to $\mathrm{PIP}_{2}$. Moreover, the changes were substantiated by an increased sensitivity to proteolysis, indicating a weakening of the tight N-ERMAD/C-ERMAD complex. ${ }^{[161]}$ In a previous study, we could confirm that the combination of $\mathrm{PIP}_{2}$ binding and phosphorylation leads to a significant increase in F-actin surface coverage of ezrin covered solid-supported lipid bilayers (SLBs). ${ }^{[14]}$

In this work, we aimed at elucidating the conformational change of ezrin upon activation by using reflectometric interference spectroscopy (RIfS) and atomic force microscopy (AFM). The increase in optical thickness (OT), i.e., the product of refractive index and physical layer thickness, over an area of $1 \mathrm{~mm}^{2}$ is detected in our RIfS setup, whereas AFM allows to resolve height levels in the subnanometer range. To map the influence of the individual activation step, ezrin wildtype 
and two mutants ezrin $\mathrm{T}_{56} 6 \mathrm{~A}$ and ezrin $\mathrm{T}_{567} \mathrm{D}$ were used. In the latter one, the threonine at position 567 was substituted for an aspartate, thus mimicking a permanent phosphorylated form. Due to the replacement of threonine by alanine, ezrin $\mathrm{T}_{567} \mathrm{~A}$ is considered constitutively inactive with regard to phosphorylation and therefore used in cell experiments. ${ }^{[162-164]}$ Membrane attachment of the proteins was either achieved by binding to the natural receptor lipid $\mathrm{PIP}_{2}$ or via the $\mathrm{N}$-terminal $6 \times$ histidine $\left(\mathrm{His}_{6}\right.$ ) tag to DOGS-NTA-Ni to evaluate the influence of $\mathrm{PIP}_{2}$. SLBs were chosen as suitable membrane model systems with regard to the application of the surface sensitive techniques RIfS and AFM. We addressed the question whether the conformational change can be monitored as a function of the protein height level on SLBs.

\subsection{RESULTS}

\subsubsection{Transformation and isolation of ezrin}

The E. coli strain TOP1o was used to amplify the plasmids pEzrinWT, pEzrinT567A, and pEzrin ${ }_{56} 6 \mathrm{D}$ containing the respective cDNA of either ezrin wildtype, ezrin $\mathrm{T}_{567} \mathrm{~A}$, and ezrin $\mathrm{T}_{567} \mathrm{D}$. The plasmids were isolated as described in section 3.1.2 and sequenced to guarantee the expression of the desired protein. All plasmids exhibited the correct DNA sequence including parts coding for the $N$-terminal $\mathrm{His}_{6}$-tag as well as for the mutations $\mathrm{T}_{5} 67 \mathrm{~A}$ and $\mathrm{T}_{5} 67 \mathrm{D}$, respectively. The whole sequences can be found in the appendix (section 5.7).

The E. coli strain BL21(DE3)pLysS was used as production host. Competent cells were transformed successfully with the corresponding plasmids as outlined in section 3.1.1. Figure 5.4 A shows BL21(DE3)pLysS colonies carrying pEzrinWT with both kanamycin and chloramphenicol resistances on LB (lysogeny broth) agar plates after transformation. Similar results were obtained with pEzrinT567A and pEzrinT567D. Protein expression was carried out according to the procedure described in 3.1.4 and nickel affinity chromatography was employed to isolate and purify the desired protein from the cell lysate (section 3.1.5). Figure 5.4 B shows the SDS-PAGE gel of the mass standard and the purified ezrin elution fraction. The broad band at $\sim 81 \mathrm{kDa}$ can be assigned to ezrin wildtype. Both mutants ezrin $\mathrm{T}_{567 \mathrm{~A}}$ and $\mathrm{T}_{567} \mathrm{D}$ migrate with a similar molecular weight in SDS-PAGE gels. However, the calculated mass of ezrin amounts to $\sim 70 \mathrm{kDa}$. This apparent higher molecular weight of ezrin in SDS-PAGE gels is well documented in literature. ${ }^{\left[{ }^{142]}\right.}$ On the one hand, ezrin contains a heptaproline sequence, which is known to induce conformational restrictions, thus influencing the electrophoretic mobility. ${ }^{[6]}$ On the other hand, the high amount of charged amino acids $(38.5 \%)$ might 
cause the unusually slow migration of ezrin on SDS-PAGE gels. ${ }^{\left[{ }^{[65]}\right.}$

A

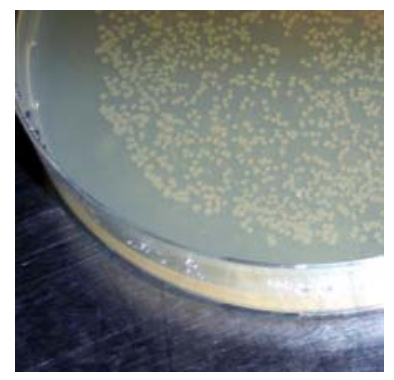

B

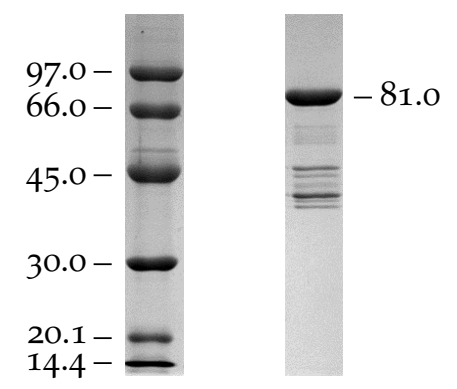

Figure 5.4: A Image showing BL21(DE3)pLysS colonies carrying pEzrinWT after successful transformation. B SDS-PAGE gels of mass standard and ezrin wildtype (wt) elution fraction stained with Coomassie Brilliant Blue. Protein band at $\sim 81 \mathrm{kDa}$ was identified as ezrin wildtype. Molecular weight in $\mathrm{kDa}$.

In addition to the broad ezrin band, there are several bands at lower molecular weight with minor intensities. It has been shown in Western blot analysis with anti$\mathrm{His}_{6}$ antibody that these contaminants can most likely be attributed to $C$-terminally truncated ezrin fragments as they are all bearing a $\mathrm{His}_{6}{ }^{- \text {tag. }}{ }^{\left[{ }^{[66]}\right.}$ Obviously, the position of the tag is not ideal with regard to purification since the protein synthesis propagates from the $\mathrm{N}$ - to the $\mathrm{C}$-terminus. However, it is essential to preserve a free $C$-terminus as the $\mathrm{F}$-actin binding site comprises the last 34 amino acids of this region. Hence, a $C$-terminal tag would probably hamper the accessibility of the F-actin binding site, which is critical with regard to the experiments in chapter 6 investigating the interaction between ezrin and F-actin. Moreover, the $\mathrm{N}$-terminal $\mathrm{His}_{6}$-tag is well suited to mimic the $N$-terminal $\mathrm{PIP}_{2}$ mediated membrane attachment of ezrin.

\subsubsection{Exploring ezrin aggregates on solid-supported lipid bilayers by RIfS}

RIfS is an optical technique allowing the label-free investigation of adsorption processes, for instance the binding of a protein to a specific receptor lipid within a solid-supported lipid bilayer (section 3.3.3.1). The adsorption process is detected as increase in optical thickness (OT), i.e., the product of refractive index and physical layer thickness.

The motivation for the following experiments originates in the question whether the different conformational states of ezrin (figure 5.3) are distinguishable in height and $O T$, respectively. We made use of ezrin wildtype, nonphosphorylatable ezrin $\mathrm{T}_{56} 6 \mathrm{~A}$, and pseudophosphorylated ezrin $\mathrm{T}_{56} 6 \mathrm{D}$. To mimic the plasma membrane attachment of ezrin, we prepared SLBs containing receptor lipids. Suitable lipids for binding of ezrin or its mutants are on the one hand the natural receptor $\mathrm{PIP}_{2}$ and on the other hand the synthetic DOGS-NTA-Ni, which is able to coordinate 
the protein via the $\mathrm{N}$-terminal $\mathrm{His}_{6}$-tag. By using these immobilization strategies in combination with the different ezrin variants, we aimed at addressing the individual contribution of the activating factors $\mathrm{PIP}_{2}$ and phosphorylation, respectively.

Receptor lipid contents of $2 \mathrm{~mol} \%$ and $4 \mathrm{~mol} \%$ were employed as we were not able to prepare SLBs exceeding a percentage of $4 \mathrm{~mol} \% \mathrm{PIP}_{2}$ at this stage. An approach overcoming this issue is given in chapter 4. Since it has been shown that $\sim 7 \mathrm{~mol} \% \mathrm{PIP}_{2}$ are required to achieve complete ezrin surface coverage of solidsupported hybrid membranes, ${ }^{[14]}$ the following $\triangle O T$ values due to ezrin adsorption are rather a qualitative measure for the conformational change of ezrin. Nevertheless, trends with regard to the conformational states of ezrin can be derived as the signal, arising from a comparably large region of $1 \mathrm{~mm}^{2}$, is averaged.

To validate the specificity of ezrin binding to $\mathrm{PIP}_{2}$ and DOGS-NTA-Ni, respectively, we prepared pure POPC-SLBs and added ezrin to control whether nonspecific binding of ezrin takes place. The spreading process of pure POPC small unilamellar vesicles (SUVs) and the addition of ezrin $\mathrm{T}_{567} \mathrm{D}$ to the formed SLB are shown in figure 5.5. The change in OT of $\sim 6.5 \mathrm{~nm}$ indicates bilayer formation upon vesicle spreading. Subsequent rinsing with E1 buffer caused no further changes in OT. The addition of o.6 $\mu \mathrm{M}$ ezrin $\mathrm{T}_{5} 67 \mathrm{D}$ (final concentration) to the pure POPC bilayer did not affect $O T$, thus showing that no ezrin binding took place.

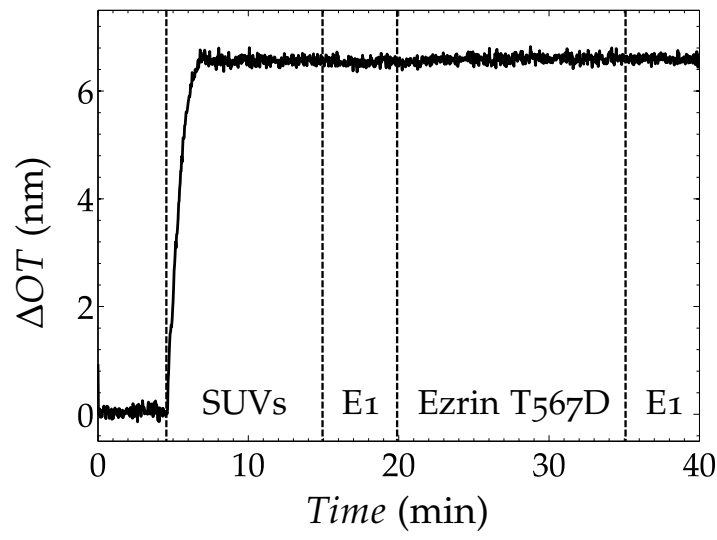

Figure 5.5: RIfS measurement controlling the specificity of ezrin binding to SLBs. Spreading of pure POPC SUVs led rapidly to bilayer formation indicated by $\triangle O T \sim 6.5 \mathrm{~nm}$. Rinsing with E1 buffer and subsequent addition of $0.6 \mu \mathrm{M}$ ezrin T567D (final concentration) caused no change in $O T$, confirming that specific receptor sites are required for ezrin binding.

This finding confirmed that specific receptor sites, e.g. NTA-Ni-groups or $\mathrm{PIP}_{2}$, are required to achieve ezrin adsorption to SLBs.

The spreading of DOPC/DOGS-NTA-Ni SUVs and following bilayer formation caused a change in OT of $(6.3 \pm 0.2) \mathrm{nm}$ in case of $2 \mathrm{~mol} \%$ DOGS-NTA-Ni $(n=10)$ and $(6.4 \pm 0.2) \mathrm{nm}$ for $4 \mathrm{~mol} \%$ DOGS-NTA-Ni $(n=6)$. Assuming that the refractive index of the lipid bilayer amounts to $1.5,{ }^{[125]}$ a physical layer thicknesses of $\sim 4.2 \mathrm{~nm}$ was calculated, which is in good agreement with literature reporting a thickness of $4.1 \mathrm{~nm}$ for a DOPC bilayer at room temperature. ${ }^{[167]}$ Representative 
binding curves of ezrin wildtype, ezrin $\mathrm{T}_{567} \mathrm{~A}$, and ezrin $\mathrm{T}_{567} \mathrm{D}$ to DOPC/DOGSNTA-Ni (96:4) are depicted in figure 5.6. The steps a and b indicate the SLB formation and subsequent rinsing with E1 buffer to adjust the conditions for protein binding, respectively. Upon addition of $\sim 0.6 \mu \mathrm{M}$ protein (step c), a distinct change in $O T$ is monitored, which is characteristic for the adsorption of a protein. Rinsing with E1 buffer (step d) caused a slight decrease in OT, which can be attributed to the removal of loosely bound protein. The actual $\triangle O T$ caused by protein binding can be extracted from the difference between the final $\triangle O T$ value (d) and the value obtained after bilayer formation (b).
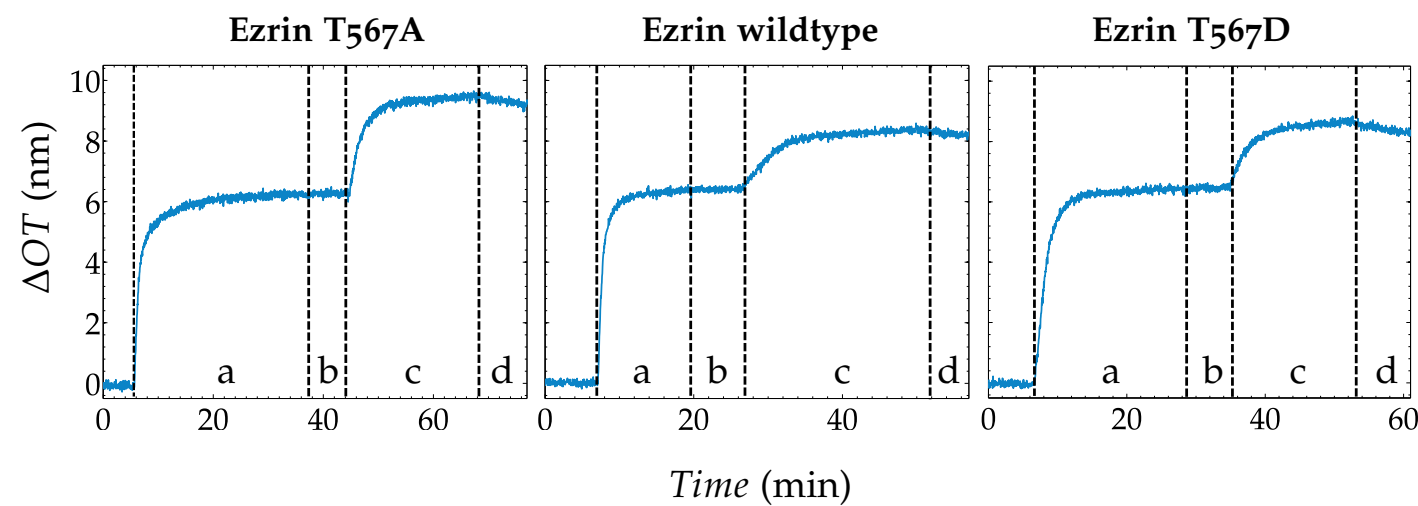

Figure 5.6: Representative binding curves of ezrin $\mathrm{T}_{567} \mathrm{~A}$, ezrin wildtype, and ezrin $\mathrm{T}_{567 \mathrm{D}}$ to SLBs composed of DOPC/DOGS-NTA-Ni (96:4) obtained by RIfS. Spreading of SUVs is indicated by a. Bilayer formation is completed when no further changes in OT are observed and $\triangle O T \sim 6 \mathrm{~nm}$ is reached. Rinsing with E1 buffer removes excess vesicles $(\mathbf{b})$. Binding of $0.6 \mu \mathrm{M}$ protein (c) and subsequent rinsing with E1 buffer (d) to evaluate the final $\triangle O T$ owing to protein binding.

All proteins produced a substantial increase in OT by binding to DOGS-NTA-Nicontaining SLBs, which was most pronounced in case of ezrin $\mathrm{T}_{5} 67 \mathrm{~A}$ and similar for ezrin wildtype and ezrin T567D. The binding curves of ezrin to DOPC/DOGSNTA-Ni (98:2) exhibited similar characteristics, although lower $\triangle O T$ values were determined owing to the lower receptor density. All results are summarized in table 5.1.

Table 5.1: Summarized values of changes in optical thickness $\triangle O T$ upon binding of ezrin to DOGS-NTA-Ni-containing SLBs with varying receptor lipid content. Mean $\langle\Delta O T\rangle$, respective standard deviations (std), and the number $n$ of experiments are given.

\begin{tabular}{|c|c|c|c|c|c|c|}
\hline & \multicolumn{3}{|c|}{$2 \mathrm{~mol} \%$ receptor } & \multicolumn{3}{|c|}{$4 \mathrm{~mol} \%$ receptor } \\
\hline & $\langle\Delta O T\rangle(\mathrm{nm})$ & \pm std (nm) & $n$ & $\langle\Delta O T\rangle(\mathrm{nm})$ & \pm std (nm) & $n$ \\
\hline Ezrin T567A & 1.6 & 0.5 & 3 & 2.8 & 0.3 & 2 \\
\hline Ezrin wildtype & 1.2 & 0.3 & 3 & 2.3 & 0.4 & 2 \\
\hline Ezrin $\mathrm{T}_{567} \mathrm{D}$ & 1.1 & 0.5 & 4 & 2.0 & 0.3 & 2 \\
\hline
\end{tabular}


Analogous to DOGS-NTA-Ni-containing SLBs, membranes composed of POPC/ $\mathrm{PIP}_{2}$ with either $2 \mathrm{~mol} \%$ or $4 \mathrm{~mol} \% \mathrm{PIP}_{2}$ were prepared. For the system with $2 \mathrm{~mol} \%$ $\mathrm{PIP}_{2}$, an increase in OT of $(6.3 \pm 0.5) \mathrm{nm}(n=12)$ was determined by RIfS, while a $\triangle O T$ of $(6.2 \pm 0.4) \mathrm{nm}(n=10)$ was found for $4 \mathrm{~mol} \% \mathrm{PIP}_{2}$. The calculated bilayer thickness amounts to $4.1 \mathrm{~nm}$ in good agreement with the bilayer thickness of $(3.98 \pm 0.08) \mathrm{nm}$ for a POPC bilayer, determined via simultaneous analysis of small angle neutron and X-ray scattering data. ${ }^{[126]}$ The slightly higher bilayer thickness in our case is most probable due to the incorporation of $\mathrm{PIP}_{2}$, whose head group is assumed to protrude from the lipid bilayer up to (5.99 \pm 1.26$) \AA$ with respect to the bridging phosphate diester. ${ }^{[87]}$ Representative binding curves of ezrin wildtype, ezrin $\mathrm{T}_{567} \mathrm{~A}$, and ezrin $\mathrm{T}_{56} 6 \mathrm{D}$ to $\mathrm{POPC} / \mathrm{PIP}_{2}$ (96:4) are shown in figure 5.7. The steps $a$ and $b$ indicate the SLB formation and subsequent rinsing with E1 buffer to adjust the conditions for protein binding. Upon addition of $\sim 0.6 \mu \mathrm{M}$ protein (step c), a distinct increase in $O T$ is monitored, which is characteristic for the adsorption of a protein. The final $\triangle O T$ caused by protein binding was determined after rinsing with E1 buffer (step d). The slight decrease in OT can be attributed to the removal of loosely bound protein.

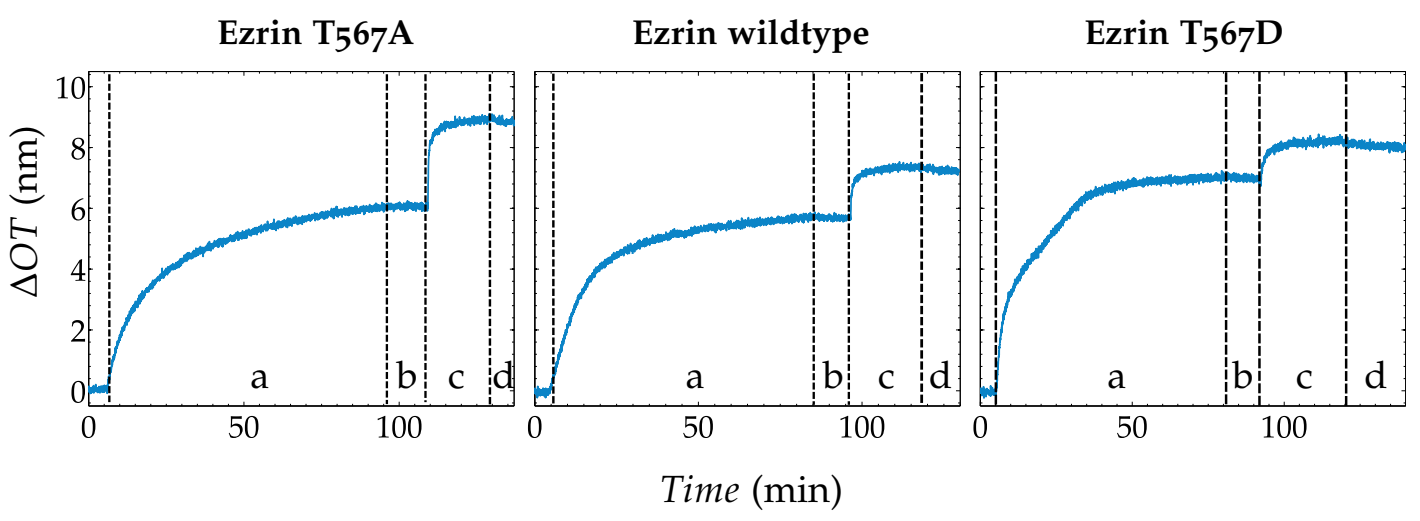

Figure 5.7: Representative binding curves of ezrin $\mathrm{T}_{5} 67 \mathrm{~A}$, ezrin wildtype, and ezrin $\mathrm{T}_{567 \mathrm{D}}$ to SLBs composed of POPC/PIP 2 (96:4) obtained by RIfS. Spreading of SUVs is indicated by a. Bilayer formation is completed when no further changes in OT are observed and $\triangle O T \sim 6 \mathrm{~nm}$ is reached. Rinsing with E1 buffer removes excess vesicles (b). Binding of $0.6 \mu \mathrm{M}$ protein (c) and subsequent rinsing with E1 buffer (d) to evaluate the final $\triangle O T$ owing to protein binding.

Notably, the determined $\triangle O T$ due to specifically bound protein differed between ezrin wildtype, ezrin $\mathrm{T}_{56} 6 \mathrm{~A}$, and ezrin $\mathrm{T}_{56} 6 \mathrm{D}$. Apparently, the greatest change in OT was observed upon adsorption of ezrin $\mathrm{T}_{5} 67 \mathrm{~A}$, whereas ezrin wildtype produced less increase in OT. Interestingly, the lowest increase was found for ezrin $\mathrm{T}_{567} \mathrm{D}$. Similar characteristics were obtained in case of ezrin binding to POPC/PIP (98:2). However, lower $\triangle O T$ values were determined owing to the lower receptor density. The summarized values are given in table 5.2. 
Table 5.2: Summarized values of changes in optical thickness $\triangle O T$ upon binding of ezrin to $\mathrm{PIP}_{2}$-containig SLBs with varying receptor lipid content. Mean $\langle\Delta O T\rangle$, respective standard deviations (std), and the number $n$ of experiments are given.

\begin{tabular}{l|ccccccc}
\hline & \multicolumn{3}{|c}{ 2 mol\% receptor } & \multicolumn{3}{c}{ 4 mol\% receptor } \\
& $\langle\Delta O T\rangle(\mathbf{n m})$ & \pm 土td (nm) & $\boldsymbol{n}$ & $\langle\Delta O T\rangle(\mathbf{n m})$ & \pm 土std (nm) & $n$ \\
\hline Ezrin T567A & 1.5 & 0.2 & 4 & 2.5 & 0.6 & 4 \\
\hline Ezrin wildtype & 1.1 & 0.2 & 4 & 2.0 & 0.5 & 3 \\
\hline Ezrin T567D & 0.6 & 0.2 & 4 & 1.1 & 0.4 & 3 \\
\hline
\end{tabular}

In the following, we summarize and compare the obtained $\triangle O T$ values as a function of receptor lipid and protein to evaluate the activation factors of ezrin, $\mathrm{PIP}_{2}$ binding and phosphorylation. Figure 5.8 shows the summarized $\triangle O T$ values upon binding of ezrin wildtype, ezrin $\mathrm{T}_{567} \mathrm{~A}$, and ezrin $\mathrm{T}_{567} \mathrm{D}$ to SLBs containing either DOGS-NTA-Ni (A) or $\mathrm{PIP}_{2}(\mathrm{~B})$ as receptor lipid. The increased receptor concentration from $2 \mathrm{~mol} \%$ to $4 \mathrm{~mol} \%$ led in both cases to an increase in the observed $\triangle O T$, indicating a higher protein coverage due to the larger number of binding sites.

Comparison of the changes in OT generated upon binding of ezrin wildtype, ezrin T567A, and ezrin T567D to DOGS-NTA-Ni-containing SLBs allows to investigate the influence of pseudophosphorylation on the conformational state separately. We observed for $2 \mathrm{~mol} \%$ receptor content similar $\triangle O T$ values for both ezrin wildtype and ezrin $\mathrm{T}_{567} \mathrm{D}$. A slightly higher increase in OT was caused by binding of ezrin T567A. The same trend was observable with $4 \mathrm{~mol} \%$ receptor lipid within the range of the standard deviation, although $\langle\Delta O T\rangle$ for ezrin $\mathrm{T}_{56} 6 \mathrm{D}$ was slightly shifted to lower values as compared to ezrin wildtype. Consequently, no clear effect of the pseudophosphorylation on the $\triangle O T$ value could be derived. Apparently, ezrin $T_{567} \mathrm{D}$ adopts a similar arrangement on the SLB surface as ezrin wildtype. Notably, ezrin T567A exhibited larger $\triangle O T$ values, even though it is expected to behave similar to ezrin wildtype. 


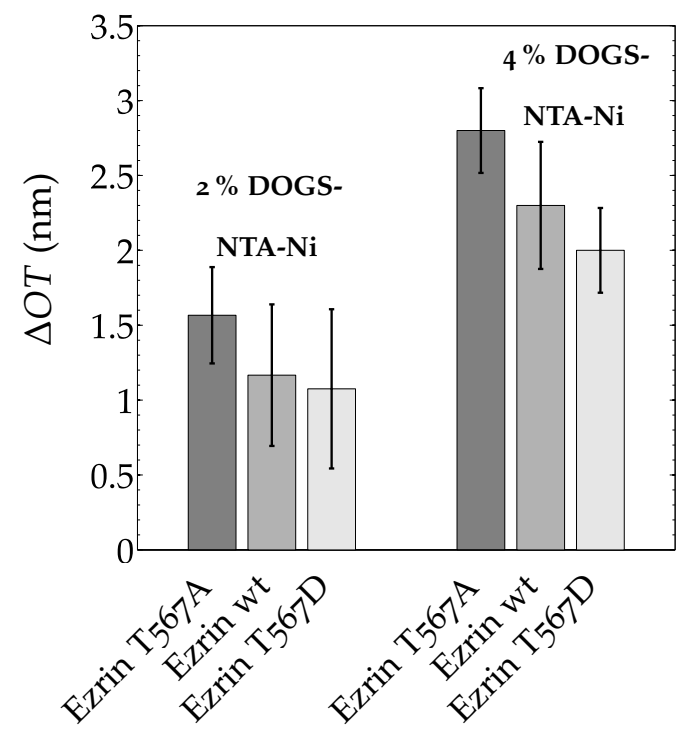

B

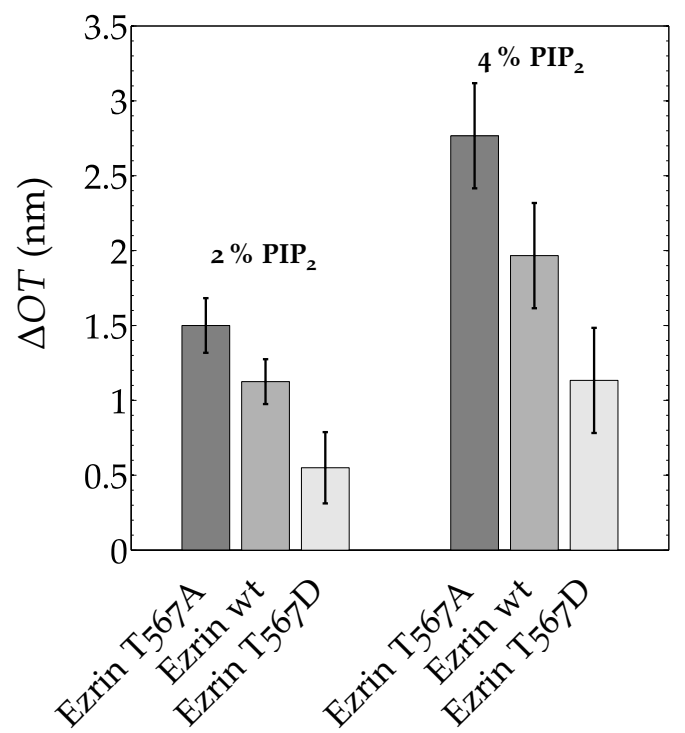

Figure 5.8: Bar diagrams illustrating $\triangle O T$ upon ezrin binding to SLBs. A Binding of ezrin $\mathrm{T}_{567 \mathrm{~A}}$, ezrin wildtype (wt), and ezrin T567D to SLBs containing either $2 \mathrm{~mol} \%$ or $4 \mathrm{~mol} \%$ DOGS-NTA-Ni (A) and PIP $_{2}($ B), respectively.

The situation changed as soon as the natural receptor lipid $\mathrm{PIP}_{2}$ came into play. Although increasing the receptor concentration from $2 \mathrm{~mol} \%$ to $4 \mathrm{~mol} \% \mathrm{PIP}_{2}$ caused a larger increase in OT - a similar behavior as in case of DOGS-NTA-Ni-containing SLBs - different $\triangle O T$ values due to binding of either ezrin wildtype, ezrin $\mathrm{T}_{5} 67 \mathrm{~A}$, or ezrin $\mathrm{T}_{5} 67 \mathrm{D}$ to $\mathrm{PIP}_{2}$-containing bilayers were obtained. Apparently, ezrin $\mathrm{T}_{56} \mathrm{~A}$ generated the largest change in $O T$, whereas lower values were determined for ezrin wildtype. Interestingly, the combination of pseudophosphorylation and $\mathrm{PIP}_{2}$ binding, ezrin $\mathrm{T}_{5} 67 \mathrm{D}$ bound to $\mathrm{PIP}_{2}$-containing SLBs, caused a considerably lower increase in OT. This trend was observable for both $2 \mathrm{~mol} \%$ and $4 \mathrm{~mol} \% \mathrm{PIP}_{2}$.

To gain an impression on the influence of $\mathrm{PIP}_{2}$, we compared $\triangle O T$ values obtained for ezrin binding to either DOGS-NTA-Ni- or $\mathrm{PIP}_{2}$-containing SLBs with $4 \mathrm{~mol} \%$ receptor lipid. A decrease of $7 \%$ was determined for the mean $\langle\Delta O T\rangle$ in case of ezrin $\mathrm{T}_{5} 67 \mathrm{~A}$ in presence of $\mathrm{PIP}_{2}$, while a shift of $13 \%$ was found for ezrin wildtype. Strikingly, the mean $\langle\Delta O T\rangle$ was reduced by $45 \%$ in case of ezrin $T_{5} 67 \mathrm{D}$ bound to the $\mathrm{PIP}_{2}$-containing SLB. Obviously, the combination of $\mathrm{PIP}_{2}$ binding and pseudophosphorylation leads to a clearly distinguishable protein arrangement on SLBs, which is characterized by lower $\triangle O T$ values. Correlating the OT qualitatively to the protein's layer thickness gives rise to the assumption that aggregates composed of ezrin $\mathrm{T}_{56} 6 \mathrm{D}$ bound to $\mathrm{PIP}_{2}$ are lower in height than ezrin wildtype or ezrin T567A clusters. Accordingly, the lower height might be an indicator for the conformational change occurring upon ezrin activation due to $\mathrm{PIP}_{2}$ and phosphorylation. 


\subsubsection{Evaluating height level and morphology of ezrin aggregates by AFM}

In RIfS, we obtained a qualitative measure for the different height levels of ezrin wildtype, ezrin $\mathrm{T}_{5} 67 \mathrm{~A}$, and ezrin $\mathrm{T}_{5} 67 \mathrm{D}$ on $\mathrm{PIP}_{2^{-}}$and DOGS-NTA-Ni-containing SLBs, respectively. To obtain quantitative height values of ezrin aggregates on SLBs, we chose to employ AFM to resolve height levels in the subnanometer range. DPPC was chosen as matrix lipid as it is in the gel phase at room temperature owing to the saturated acyl chains. Accordingly, DPPC-SLBs are rather stiff and not as deformable as SLBs composed of fluid lipids such as POPC. This property is in particular advantageous when using AFM imaging to determine topographic features formed by protein aggregates immobilized to such a membrane. To evaluate the influence of the gel phase matrix on the distribution of $\mathrm{PIP}_{2}$, we used $\mathrm{PIP}_{2}$-containing POPC-SLBs for comparison.

\section{Membrane integrity}

Each SLB used for protein immobilization was characterized before by AFM imaging. Bodipy- $\mathrm{C}_{12}-\mathrm{HPC}$ had been added at $0.5 \mathrm{~mol} \%$ to the lipid mixture to validate bilayer formation by fluorescence spectroscopy before the AFM measurement. Figure 5.9 shows representative height images obtained for POPC/PIP $2 /$ Bodipy- $\mathrm{C}_{12}$ HPC (96.5:3:0.5) SLBs (A) and DPPC/PIP 2 /Bodipy-C 12 -HPC (96.5:3:0.5) bilayers (B). Both membranes are characterized by a smooth membrane surface (yolk colored). Black areas represent membrane defects with depths of $\sim 4 \mathrm{~nm}$ in case of POPCSLBs $\left(\mathrm{A}^{\prime}\right)$ and $\sim 5 \mathrm{~nm}$ for DPPC-containing bilayers $\left(\mathrm{B}^{\prime}\right)$ according to the single line profiles. The average defect depth is in agreement with the bilayer thickness of $(3.98 \pm 0.08) \mathrm{nm}$ for a POPC bilayer, determined via simultaneous analysis of small angle neutron and X-ray scattering data ${ }^{[126]}$ and the thickness obtained for a DPPC SLB of $\sim 5.1 \mathrm{~nm}$ determined by AFM. ${ }^{[168]}$ Therefore, we concluded that single bilayers were formed upon spreading of SUVs containing either POPC/PIP ${ }_{2} /$ Bodipy$\mathrm{C}_{12}$-HPC (96.5:3:0.5) or DPPC/PIP 2 /Bodipy-C ${ }_{12}$-HPC (96.5:3:0.5). Notably, DPPCcontaining membranes are characterized by many defects, while POPC-SLBs are rather homogeneous. This can be attributed to the so-called self-healing properties of fluid lipid bilayers on smooth hydrophilic surfaces. ${ }^{[169]}$ Accordingly, defects are only present if topological faults in the solid surface are generated due to mechanical scratching. In contrast, DPPC is in the gel phase at room temperature forming a stiffer bilayer, which is not able to re-seal defects due to the decreased lateral lipid mobility. With regard to a possible inhomogeneous $\mathrm{PIP}_{2}$ distribution within DPPCSLBs due to phase demixing of the gel phase lipid DPPC and the fluid PIP ${ }_{2}$, we controlled the distribution of the fluorescent $\mathrm{PIP}_{2}$ analogue Bodipy TMR-PIP ${ }_{2}$ within 
a DPPC matrix by CLSM. Based on its homogeneous fluorescence, we concluded that $\mathrm{PIP}_{2}$ was homogeneously distributed within the DPPC-SLB with respect to the resolution limit of the CLSM. ${ }^{[170]}$ Moreover, AFM imaging of DPPC-SLBs with varying $\mathrm{PIP}_{2}$ (up to $7 \mathrm{~mol} \%$ ) yielded in all cases smooth membrane surfaces.
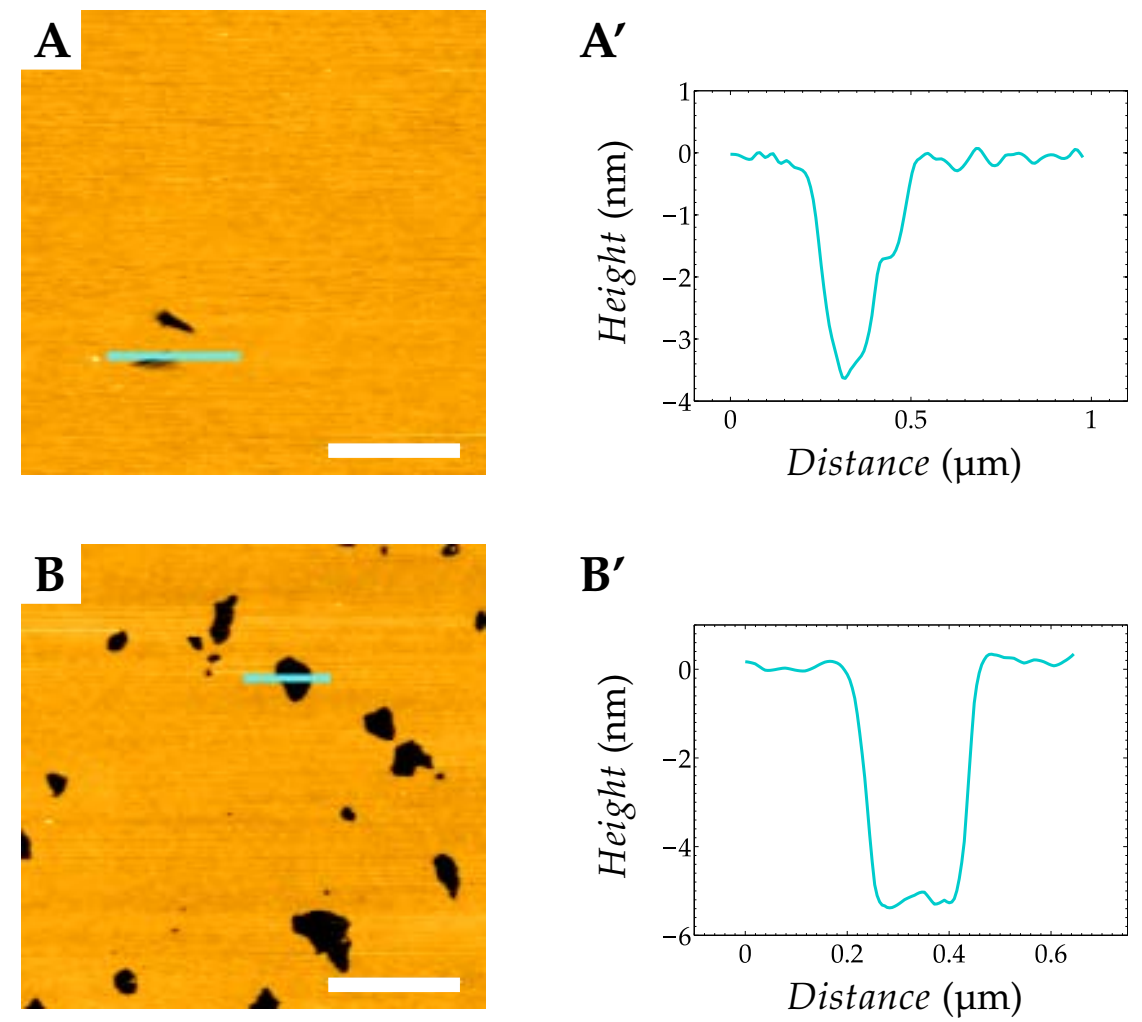

Figure 5.9: Representative AFM images (tapping mode) of SLBs composed of either POPC/PIP 2 /Bodipy-C ${ }_{12}$-HPC (96.5:3:0.5) (A) or DPPC/PIP 2 /Bodipy-C ${ }_{12}-$ HPC (96.5:3:0.5) (B) on silicon support in E1 buffer. Defects of $\sim 4 \mathrm{~nm}$ in POPC-containing SLBs $\left(\mathbf{A}^{\prime}\right)$ and defects in DPPC-SLBs of $\sim 5 \mathrm{~nm}\left(\mathbf{B}^{\prime}\right)$ are consistent with the thickness of a single bilayer. Single line profiles correspond to turquoise lines in $\mathbf{A}$ and $\mathbf{B}$ to validate membrane formation. Scale bars: $1 \mu \mathrm{m}$.

Ezrin binding to DPPC bilayers via PIP ${ }_{2}$ or DOGS-NTA-Ni

Similar to the RIfS measurements, we increased the PIP ${ }_{2}$ content within DPPC-SLBs to monitor the increasing ezrin surface coverage by AFM imaging. $\mathrm{PIP}_{2}$ percentages of $3 \mathrm{~mol} \%, 5 \mathrm{~mol} \%$, and $7 \mathrm{~mol} \%$ were employed. The spreading protocol employing low $\mathrm{pH}$, developed in chapter 4 , was used for the preparation of $\mathrm{PIP}_{2}$-containing SLBs. Figure 5.10 shows the adsorption of ezrin wildtype to SLBs with increasing $\mathrm{PIP}_{2}$ percentages. In general, three different height levels were observed after incubation with ezrin as can be seen in single line profiles $\left(\mathrm{A}^{\prime}-\mathrm{C}^{\prime}\right)$ and by the AFM color scale (A-C). Dark brown or black areas correspond to membranes defects as can be deduced from their shape and depth. Middle brown colored regions represent the membrane surface, whereas orange-brown colored features correspond to bound 
ezrin. Very bright, circular structures are mostly due to adhered excess vesicles, exhibiting heights in the range of $10 \mathrm{~nm}$ to $30 \mathrm{~nm}$.

All SLBs were covered with widely distributed, elongated protein aggregates after incubation with ezrin wildtype as indicated by the orange-brown colored structures (5.10 A-C).
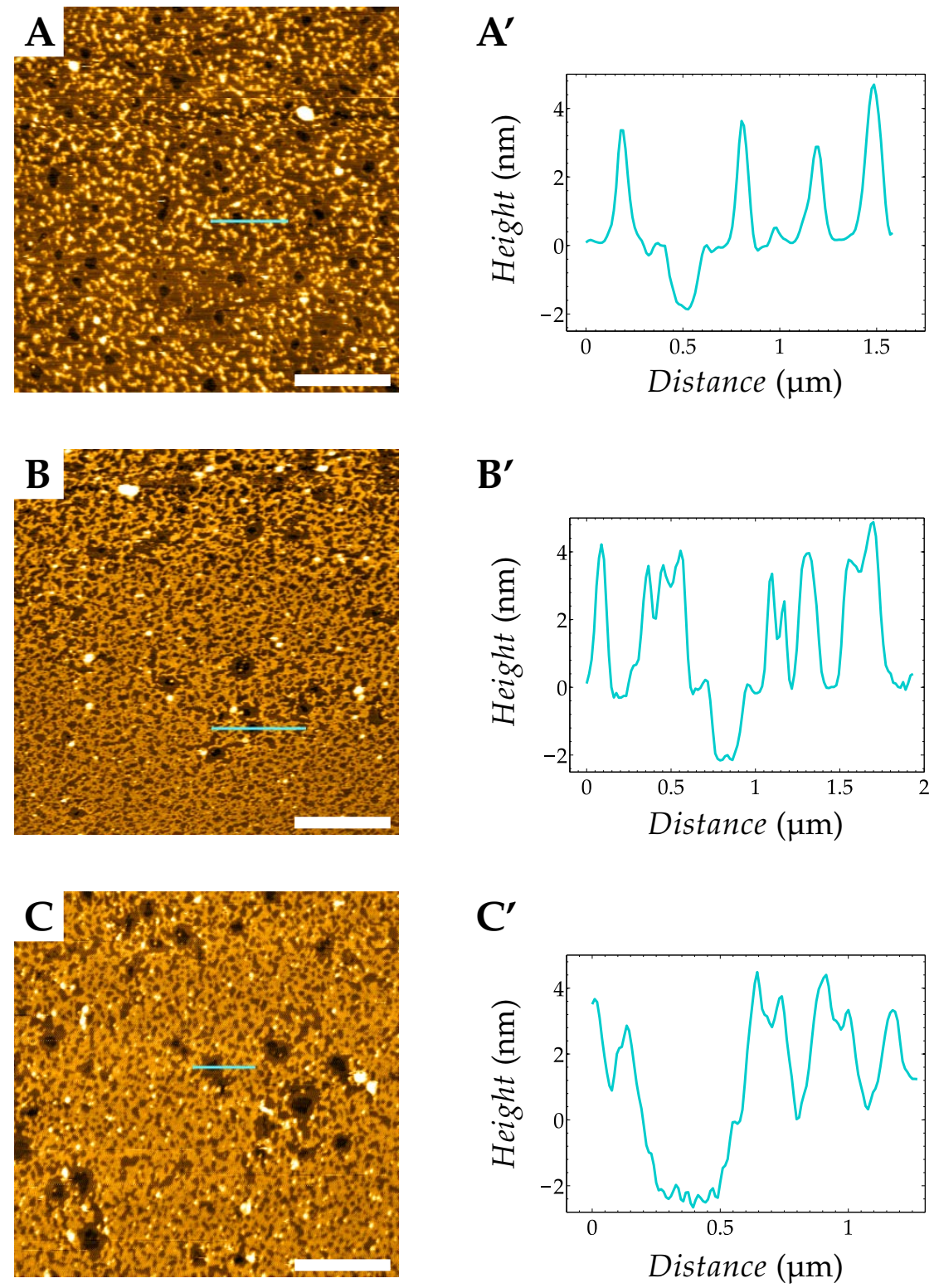

Figure 5.10: Representative AFM images (tapping mode) of DPPC $/ \mathrm{PIP}_{2} /$ Bodipy- $\mathrm{C}_{12}-\mathrm{HPC}$ SLBs with varying $\mathrm{PIP}_{2}$ content on silicon support in E1 buffer. SLBs containing $3 \mathrm{~mol} \%$ (A), $5 \mathrm{~mol} \%(\mathbf{B})$, and $7 \mathrm{~mol} \%$ (C) $\mathrm{PIP}_{2}$ after incubation with $0.6 \mu \mathrm{M}$ ezrin wildtype (final concentration). Single line profiles $\left(\mathbf{A}^{\prime}-\mathbf{C}^{\prime}\right)$ correspond to turquoise lines in $\mathbf{A}-\mathbf{C}$ showing protein cluster heights ranging from $3 \mathrm{~nm}$ to $4 \mathrm{~nm}$. Scale bars: $2 \mu \mathrm{m}$.

As expected, the highest ezrin surface coverage was observed with $7 \mathrm{~mol} \% \mathrm{PIP}_{2}$ (C). Only few areas can be attributed to defects (dark brown) and uncovered membrane surface (middle brown). The ezrin wildtype aggregates exhibit a height level ranging from $3 \mathrm{~nm}$ to $4 \mathrm{~nm}$ as illustrated in the single line profiles $\left(\mathrm{A}^{\prime}-\mathrm{C}^{\prime}\right)$. Mem- 
brane defects were still detectable, although it appeared that protein material has partially filled these holes since the depth is reduced to $\sim 2 \mathrm{~nm}$.

The effect of increasing $\mathrm{PIP}_{2}$ concentration on the ezrin surface coverage was further evaluated by pixel analysis. For $3 \mathrm{~mol} \%$ a surface coverage ranging from $20 \%$ to $28 \%$ was found, whereas $40 \%$ to $52 \%$ of SLBs containing $5 \mathrm{~mol} \% \mathrm{PIP}_{2}$ were covered with protein. The highest ezrin surface coverage was obtained with $40 \%$ to $57 \%$ for $7 \mathrm{~mol}^{2} \mathrm{PIP}_{2}$ content.

Histogram height analysis is usually used to evaluate the distribution of height levels in topographic AFM images (5.11 A). All height values originating from an AFM height image (or a selection) are pooled in a single histogram. However, in case of low protein coverage and small protein clusters, the protein height level is often only visible as shoulder in the histogram. Most of our AFM images revealed to be challenging with regard to conventional histogram analysis. Therefore, we established the histogram height analysis tool based in MATLAB. Figure 5.11 B shows schematically the process: (1) the height level of the membrane surface is set to zero for each image to obtain comparable height values for protein aggregates. (2) Threshold setting allows to exclude height values arising from membrane defects, the membrane surface, and artifacts caused by data flattening. Overlays of real image and analyzed image were generated to control the chosen threshold.

A
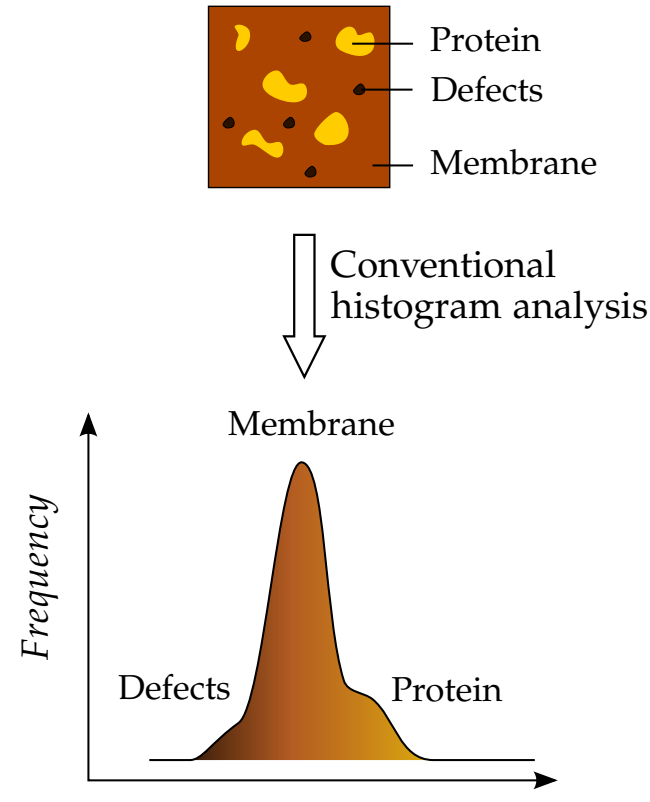

Height $(\mathrm{nm})$
B

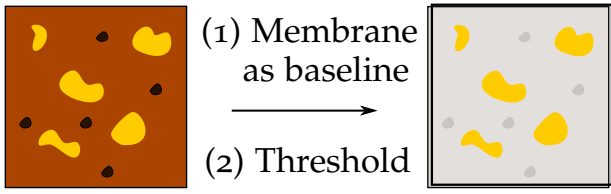
$\sqrt{\begin{array}{l}\text { Histogram height } \\ \text { analysis tool }\end{array}}$

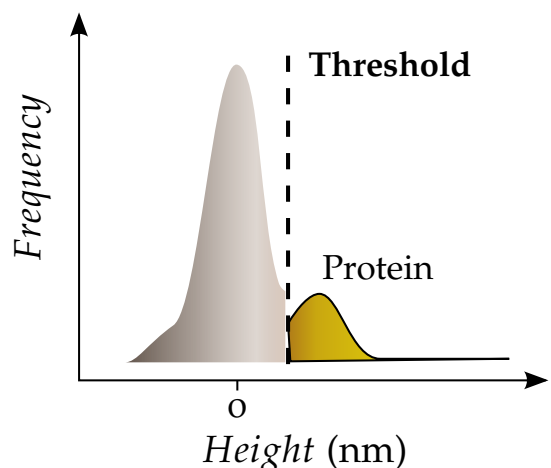

Figure 5.11: Schematic drawing illustrating histogram height analysis. A Conventional histogram analysis pools height values originating from selected area of topographic image into the histogram. Height levels of defects, membrane, and protein are not clearly distinguishable in the histogram $\mathbf{B}$ The histogram height analysis tool defines the membrane height level as zero and allows to set a lower threshold to extract only relevant height values. The histogram shows only the height level of protein clusters. 
Evaluating the recorded height images by using the histogram height analysis tool, overlay images as depicted in figure 5.12 A were produced. The determined protein height values were not depending on the $\mathrm{PIP}_{2}$ content of the SLB. Consequently, all values were combined in one single histogram (figure 5.12 B). An average height of $(3.5 \pm 0.9) \mathrm{nm}$ was determined for ezrin wildtype on PIP $_{2}$-containing DPPC-SLBs.

A

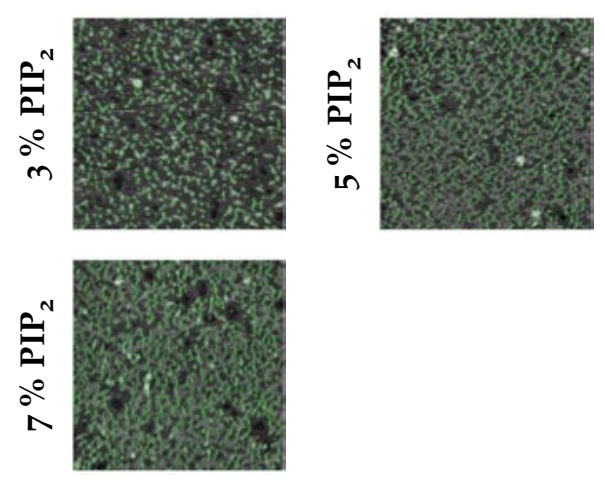

B

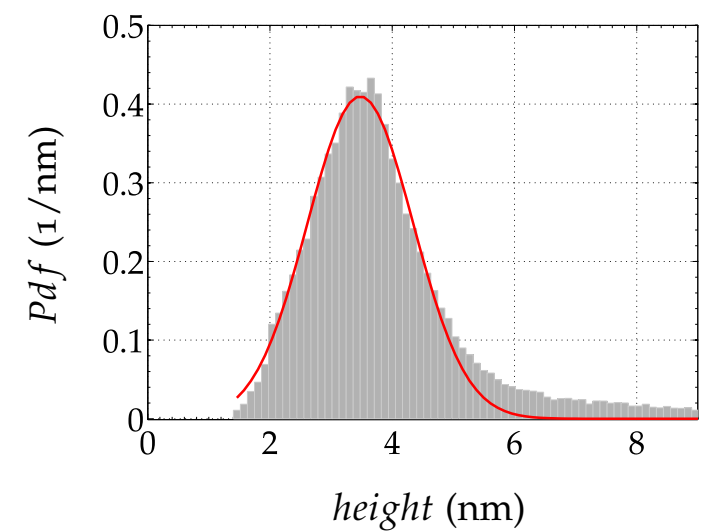

Figure 5.12: A Representative overlay images generated by the histogram height analysis tool. Green pixels correspond to maxima used for further height analysis. B Height analysis histogram of ezrin wildtype on PIP $_{2}$-containing DPPC-SLBs reveals an average height of $(3.5 \pm 0.9) \mathrm{nm}$. No correlation of protein cluster height and PIP ${ }_{2}$ content was observed. The red line represents the Gaussian fit to the data yielding the mean height level \pm standard deviation.

To evaluate the influence of $\mathrm{PIP}_{2}$ on the height level and morphology of ezrin wildtype on DPPC-SLBs, we substituted PIP ${ }_{2}$ for DOGS-NTA-Ni. The binding of ezrin wildtype to a SLB composed of DPPC/DOGS-NTA-Ni/Bodipy-C ${ }_{12}$-HPC SLB (96.5:3:0.5) is depicted in figure 5.13 A. Notably, a completely different pattern of protein arrangement on the membrane surface was observed. Instead of elongated, reticular structures covering large parts of the surface, it appears that a rather spotlike adsorption of ezrin wildtype to DOGS-NTA-Ni generated smaller, roundish elevated structures on the membrane surface. The single line profile $\left(\mathrm{A}^{\prime}\right)$ revealed a protein height level ranging from $1.5 \mathrm{~nm}$ to $2.5 \mathrm{~nm}$, while the membrane defect showed a reduced depth of $\sim 2 \mathrm{~nm}$ probably due to nonspecifically bound protein. The impression of a rather low ezrin surface coverage was confirmed by pixel analysis, yielding a range of $7 \%$ to $14 \%$ for the covered membrane area by bound ezrin wildtype. 

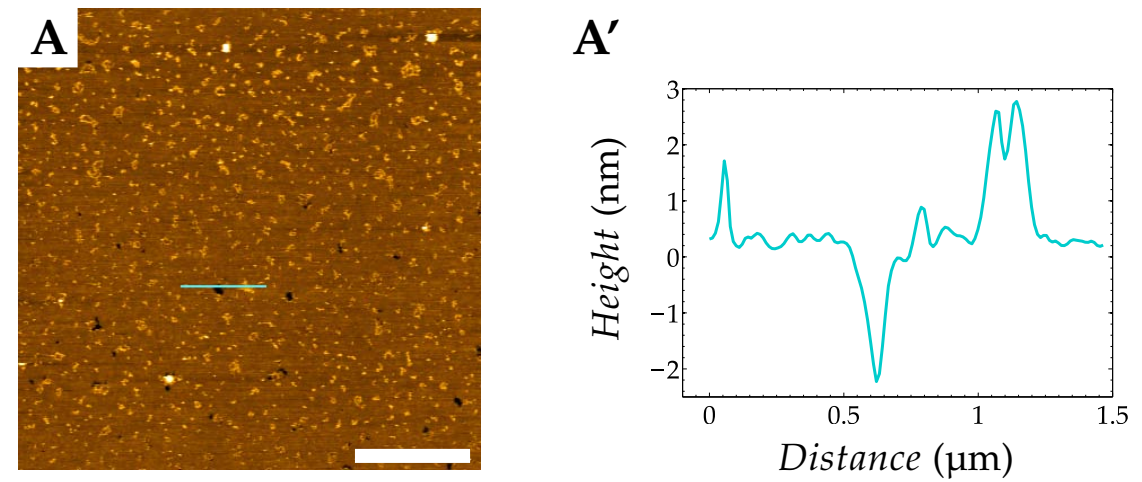

Figure 5.13: Representative AFM image (tapping mode) of DPPC/DOGS-NTA-Ni/Bodipy$\mathrm{C}_{12}$-HPC SLB (96.5:3:0.5) on silicon support in E1 buffer. A Protein covered SLB after incubation with o.6 $\mu \mathrm{M}$ ezrin wildtype (final concentration). Single line profile $\left(\mathbf{A}^{\prime}\right)$ corresponds to turquoise line in A showing protein cluster heights of $\sim 3 \mathrm{~nm}$. Scale bar: $2 \mu \mathrm{m}$.

Figure 5.14 A shows the overlay image generated by histogram height analysis tool. Ezrin wildtype exhibited height levels in the range of $(2.8 \pm 1.5) \mathrm{nm}$ on DOGS-NTANi-containing DPPC-SLBs (figure 5.14 B).

A

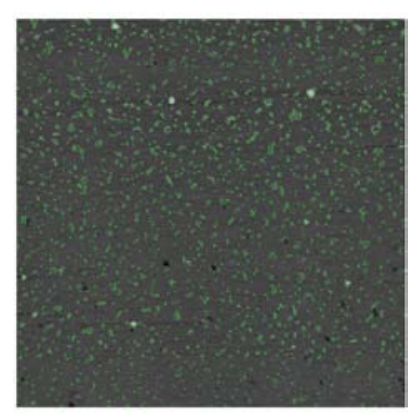

B

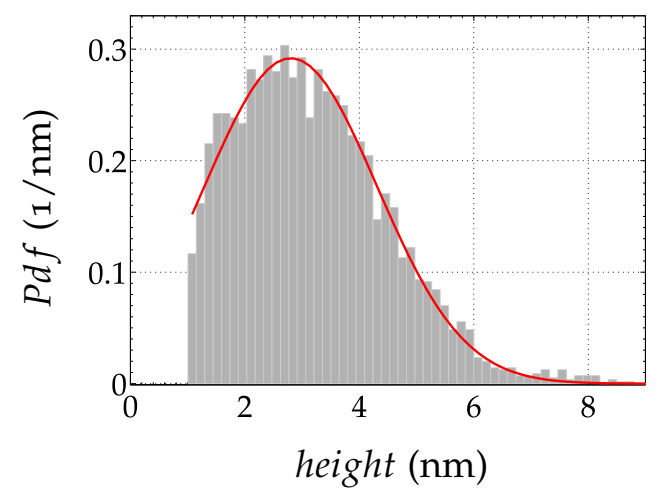

Figure 5.14: A Representative overlay image generated by the histogram height analysis tool. Green pixels correspond to maxima used for further height analysis. B Height analysis histogram of ezrin wildtype on DOGS-NTA-Ni-containing DPPC-SLBs (3 mol\%) reveals an average height of $(2.8 \pm 1.5) \mathrm{nm}$. The red line represents the Gaussian fit to the data yielding the mean height level \pm standard deviation. Height values $<1 \mathrm{~nm}$ are excluded.

The combination of both ezrin activation factors $\mathrm{PIP}_{2}$ and phosphorylation was mimicked by the binding of ezrin $\mathrm{T}_{5} 67 \mathrm{D}$ to $\mathrm{PIP}_{2}$-containing DPPC-SLBs. Figure 5.15 A shows the adsorption of ezrin $\mathrm{T}_{5} 67 \mathrm{D}$ to a SLB composed of DPPC/ $\mathrm{PIP}_{2} /$ Bodipy-C ${ }_{12}$-HPC SLBs (96.5:3:0.5). Ezrin $\mathrm{T}_{567 \mathrm{D}}$ formed similar to ezrin wildtype elongated aggregates on the $\mathrm{PIP}_{2}$-containing DPPC membrane surface (orangebrown colored). However, it appeared that these regions were lower in height, indicated by the slightly darker shade as compared to ezrin wildtype aggregates in figure 5.10 A. This was confirmed by the single line profile (figure $5.15 \mathrm{~A}^{\prime}$ ), showing height levels for protein aggregates ranging from $1.5 \mathrm{~nm}$ to $2 \mathrm{~nm}$. The depth of membrane defects was reduced to $\sim 2.5 \mathrm{~nm}$ similar to the behavior observed before for defects in $\mathrm{PIP}_{2}$-containing DPPC membranes after incubation with ezrin wild- 
type. Notably, the protein surface coverage determined via pixel analysis amounts to $38 \%$ to $52 \%$ for a $\mathrm{PIP}_{2}$ content of $3 \mathrm{~mol} \%$. This is considerably higher than the range from $20 \%$ to $28 \%$ coverage found for ezrin wildtype on similar membranes.
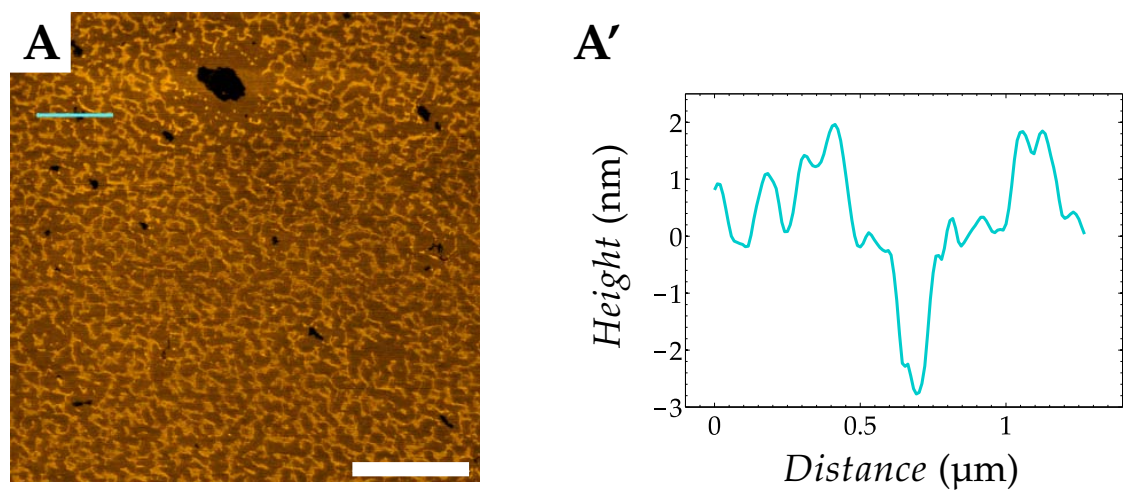

Figure 5.15: Representative AFM image (tapping mode) of DPPC $/ \mathrm{PIP}_{2} /$ Bodipy- $\mathrm{C}_{12}-\mathrm{HPC}$ SLBs (96.5:3:0.5) on silicon support in E1 buffer. A Protein covered SLB after incubation with $0.6 \mu \mathrm{M}$ ezrin $\mathrm{T}_{567} \mathrm{D}$ (final concentration). Single line profile ( $\left.\mathbf{A}^{\prime}\right)$ corresponds to turquoise line in A showing protein cluster heights of $\sim 2 \mathrm{~nm}$. Scale bar: $2 \mu \mathrm{m}$.

The overlay image produced by the histogram height analysis tool is depicted in figure $5.16 \mathrm{~A}$. In contrast to ezrin wildtype bound via $\mathrm{PIP}_{2}$, ezrin $\mathrm{T}_{567} \mathrm{D}$ was characterized by significantly lower protein aggregates in the range of $(1.5 \pm 0.8) \mathrm{nm}$ on $\mathrm{PIP}_{2}$-containing DPPC-SLBs (figure 5.16 B).

A

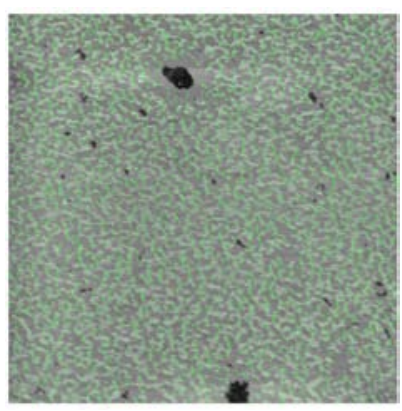

B

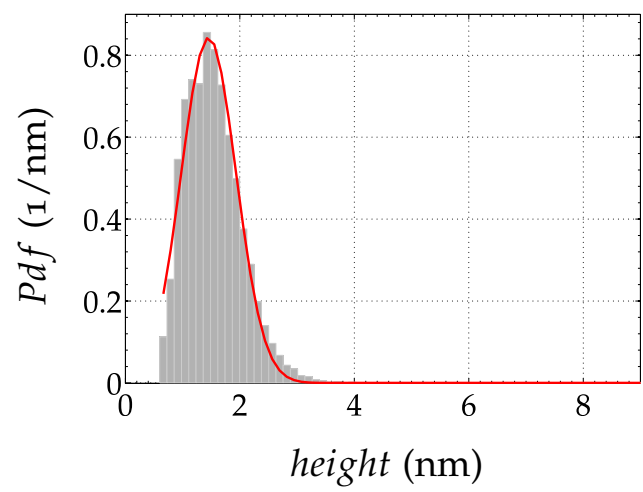

Figure 5.16: A Representative overlay image generated by the histogram height analysis tool. Green pixels correspond to maxima used for further height analysis. B Height analysis histogram of ezrin $\mathrm{T}_{5} 67 \mathrm{D}$ on $\mathrm{PIP}_{2}$-containing DPPC-SLBs $(3 \mathrm{~mol} \%)$ reveals an average height of $(1.5 \pm 0.8) \mathrm{nm}$. The red line represents the Gaussian fit to the data yielding the mean height level \pm standard deviation.

Ezrin immobilization to POPC bilayers via $\mathrm{PIP}_{2}$

Figure 5.17 shows the adsorption of ezrin wildtype to a SLB composed of POPC/ $\mathrm{PIP}_{2}$ /Bodipy- $\mathrm{C}_{12}$-HPC SLB (96.5:3:0.5). As POPC-containing SLBs exhibited only few membrane defects, two height levels were observed after protein incubation. 
Middle brown areas correspond to the membrane surface, while elevated protein structures are brown-orange colored. A remarkable change regarding the pattern of protein arrangement on the membrane was found by replacing the matrix lipid DPPC with POPC. Instead of elongated protein structures covering larges parts of the membrane surface, circular features (orange-brown) with a diameter ranging from $200 \mathrm{~nm}$ to $600 \mathrm{~nm}$ were observed, indicating the formation of round protein clusters (A, B). The single line profiles $\left(\mathrm{A}^{\prime}, \mathrm{B}^{\prime}\right)$ revealed a height level of $\sim 4 \mathrm{~nm}$ due to adsorption of ezrin wildtype to $\mathrm{PIP}_{2}$.
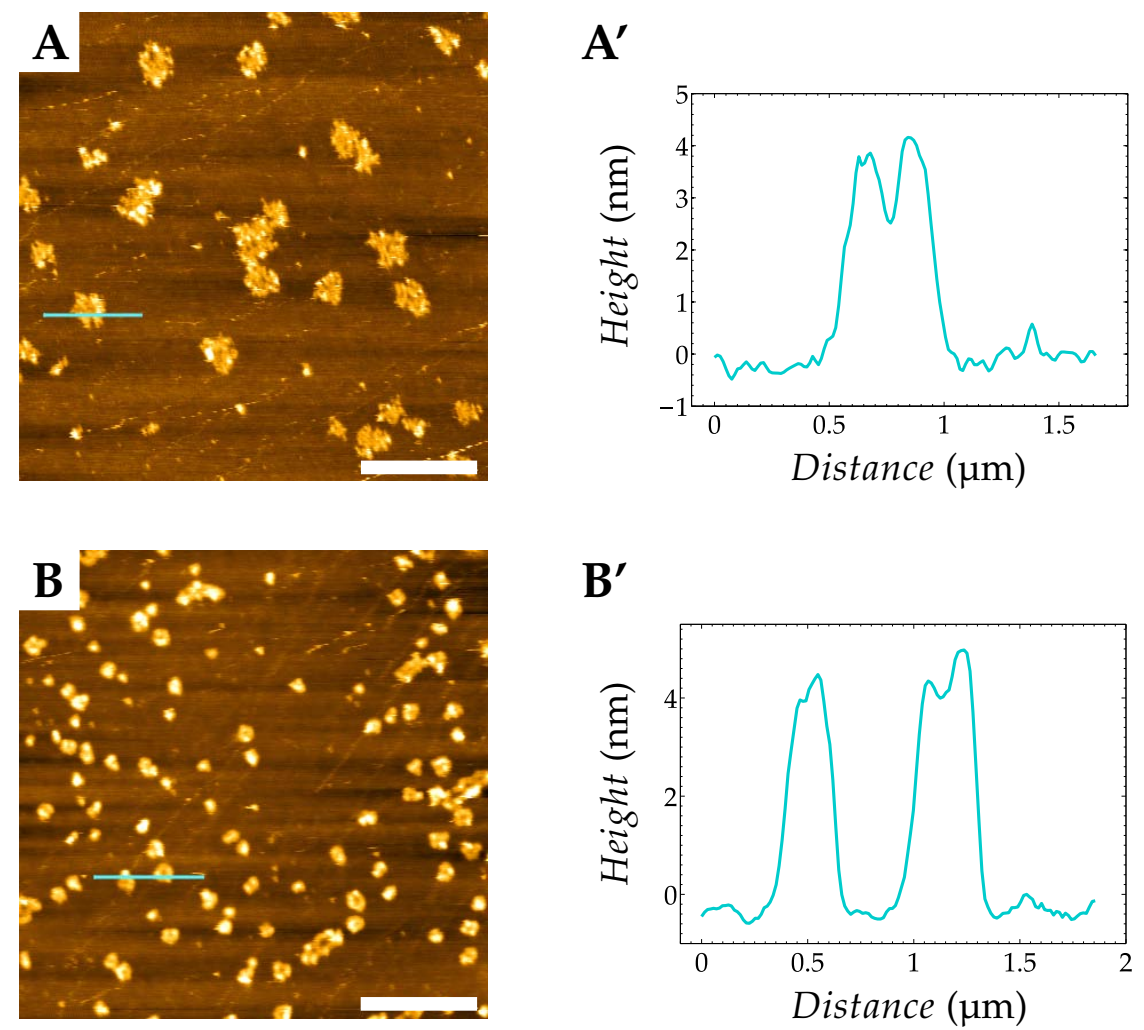

Figure 5.17: Representative AFM images (tapping mode) of POPC $/ \mathrm{PIP}_{2} /$ Bodipy- $_{12}-\mathrm{HPC}$ SLB (96.5:3:0.5) on silicon support in E1 buffer. Ezrin wildtype clusters of $\sim 0.5 \mu \mathrm{m}$ in diameter (A) and smaller ezrin wildtype aggregates of $\sim 0.3 \mu \mathrm{m}(\mathbf{B})$ were found after incubation with o.6 $\mu \mathrm{M}$ ezrin wildtype (final concentration). Single line profiles $\left(\mathbf{A}^{\prime}, \mathbf{B}^{\prime}\right)$ correspond to turquoise lines in $\mathbf{A}$ and $\mathbf{B}$ showing protein heights of $\sim 4 \mathrm{~nm}$. Scale bars: $2 \mu \mathrm{m}$.

Figure $5.18 \mathrm{~A}$ and $\mathrm{C}$ show the overlay images generated by the histogram height analysis tool. At first sight, the height level appears to be dependent on the cluster dimension as larger ones exhibited height values in the range of $(3.4 \pm 2.1) \mathrm{nm}$ (figure $5.18 \mathrm{~B}$ ), whereas smaller clusters were characterized by a height level of $(4.0 \pm 2.4) \mathrm{nm}$ (figure $5.18 \mathrm{D}$ ). However, the large error range indicates that these deviation might arise rather from imaging or flattening artifacts. The POPC matrix is considerably softer than DPPC. Accordingly, small deviations of the applied force might give rise to apparently different height levels. Moreover, flattening artifacts, i.e., horizontally or vertically arranged, stripelike features due to raw data processing, are clearly visible in figure $5.17 \mathrm{~A}$ and $\mathrm{B}$. Consequently, the height level 
of the membrane surface, which is used as point of reference for the determination of the protein height level, cannot be clearly identified, leading to rather large errors in the estimation of the protein cluster height.

A

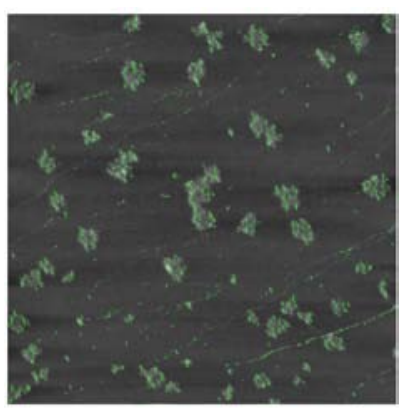

C

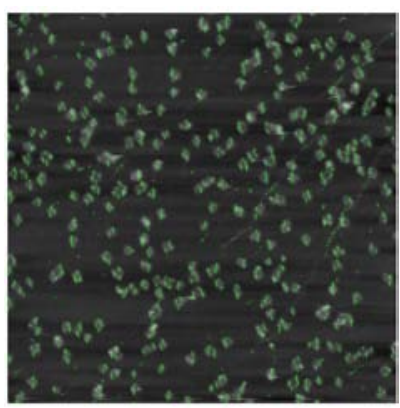

B

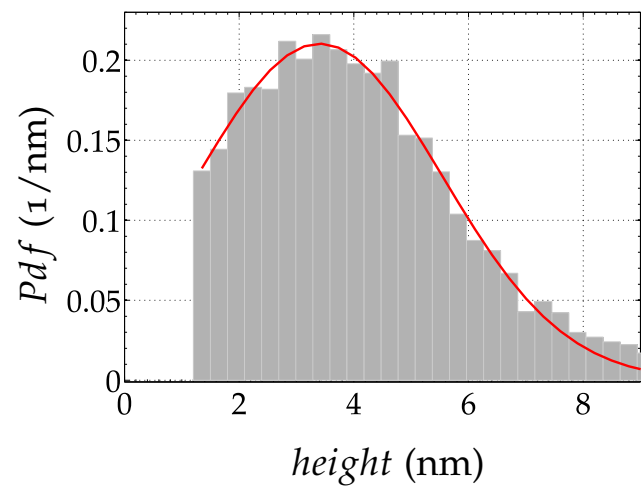

D

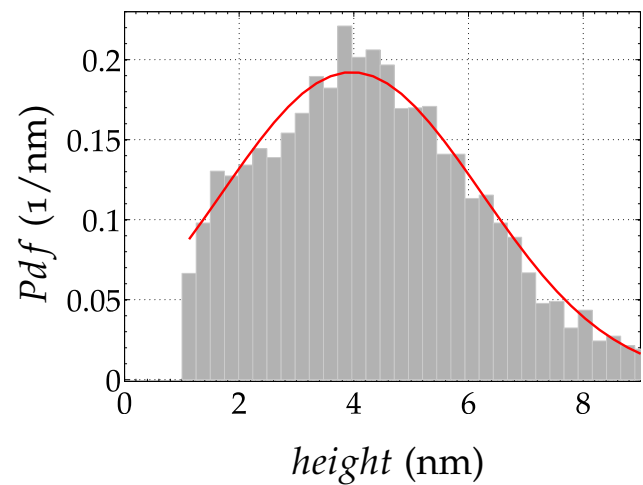

Figure 5.18: A, C Representative overlay images generated by the histogram height analysis tool. Green pixels correspond to maxima used for further height analysis. Height analysis histograms of ezrin wildtype on PIP $_{2}$-containing POPC-SLBs reveal an average height of $(3.4 \pm 2.1) \mathrm{nm}$ for larger clusters $(B)$ and $(4.0 \pm 2.4) \mathrm{nm}$ for smaller ones (D). The red lines represent the Gaussian fits to the data $\bar{x} \pm \sigma$.

All determined height levels of ezrin wildtype and ezrin $\mathrm{T}_{567} \mathrm{D}$ on different membrane systems are summarized in table 5.3. Although it appears that ezrin wildtype exhibits rather similar height levels on DPPC-SLBs independent of the receptor lipid, clear differences were observable with respect to the shape of protein clusters and the protein surface coverage. Ezrin wildtype immobilized via $\mathrm{PIP}_{2}$ formed elongated structures, covering a surface area in the range of $20 \%$ to $28 \%$, whereas the same protein bound to DOGS-NTA-Ni was characterized by a rather circular, spotlike arrangement spread over $7 \%$ to $14 \%$ of the membrane surface. This deviation can most probably be attributed to the different receptor lipids DOGS-NTA-Ni and PIP $_{2}$. With regard to the activation process of ezrin, ezrin wildtype immobilized via DOGS-NTA-Ni represents the completely inactive form, whereas partial activation is believed to occur upon binding to $\mathrm{PIP}_{2}$. This situation is mimicked by ezrin wildtype bound to $\mathrm{PIP}_{2}$. However, $\mathrm{PIP}_{2}$ appears 
to affect rather the protein arrangement on the membrane surface than the actual height level. Full activation is proposed to be achieved by $\mathrm{PIP}_{2}$ binding and subsequent phosphorylation of the threonine at position 567. This case is represented by ezrin $\mathrm{T}_{56}{ }_{7} \mathrm{D}$ bound to $\mathrm{PIP}_{2}$, where we observed a clear decrease in height level and considerable increase in protein surface coverage to $38 \%$ to $52 \%$. This finding suggests that the active state of ezrin is characterized by a rather elongated, flattened shape. Notably, the more fluid matrix lipid POPC led to the formation of differently shaped ezrin wildtype aggregates in presence of $\mathrm{PIP}_{2}$. Instead of elongated structures, a rather circular protein arrangement was obtained. Obviously, the influence of the gel matrix influences the shape of protein aggregates in case of $\mathrm{PIP}_{2}$.

Table 5.3: Summary of height levels and surface coverages of ezrin clusters as a function of matrix and receptor lipid. * Average of measurements with $3 \mathrm{~mol} \%, 5 \mathrm{~mol} \%$, and $7 \mathrm{~mol} \%$ receptor. Mean values determined from smaller $(\ddagger)$ and larger $(\dagger)$ clusters.

\begin{tabular}{|c|c|c|c|c|}
\hline & Matrix lipid & Receptor lipid & $\begin{array}{c}\text { Height level } \\
(\mathrm{nm})\end{array}$ & $\begin{array}{l}\text { Surface coverage for } \\
3 \mathrm{~mol} \% \text { receptor }\end{array}$ \\
\hline \multirow{4}{*}{ Ezrin wildtype } & DPPC & $\mathrm{PIP}_{2}$ & $* 3.5 \pm 0.9$ & $20-28$ \\
\hline & DPPC & DOGS-NTA-Ni & $2.8 \pm 1.5$ & $7-14$ \\
\hline & POPC & $\mathrm{PIP}_{2}$ & ${ }^{\dagger} 3.4 \pm 2.1$ & ${ }^{+} 7-10$ \\
\hline & POPC & $\mathrm{PIP}_{2}$ & $\ddagger 4.0 \pm 2.4$ & $\ddagger_{10}-14$ \\
\hline Ezrin $\mathrm{T}_{56} 6_{7} \mathrm{D}$ & DPPC & $\mathrm{PIP}_{2}$ & $1.5 \pm 0.8$ & $38-52$ \\
\hline
\end{tabular}

\section{$5 \cdot 3$ DISCUSSION}

In this work, we investigated the activation process and the associated conformational change of ezrin, induced by $\mathrm{PIP}_{2}$ binding and phosphorylation. In particular, we addressed the question whether ezrin in different activation states - no, partial, and full activation - would exhibit different height levels when immobilized on SLBs. To map the individual influence of the particular activation factor, we employed on the one hand ezrin wildtype as well as nonphosphorylatable and pseudophosphorylated ezrin mutants, namely ezrin $\mathrm{T}_{567} \mathrm{~A}$ and ezrin $\mathrm{T}_{56} 6_{7} \mathrm{D}$. The influence of $\mathrm{PIP}_{2}$ binding was assessed as such that we made use of SLBs containing either $\mathrm{PIP}_{2}$ or DOGS-NTA-Ni as receptor lipid for ezrin binding. We employed the surface sensitive techniques RIfS and AFM to visualize the height levels caused upon protein binding to SLBs. The influence of gel phase DPPC as matrix lipid on the shape of protein clusters was examined by comparison with a fluid POPC matrix. The use of the optical RIfS technique and AFM imaging is beneficial as RIfS allows to determine height changes without manipulating influence, whereas AFM 
provides a high spatial resolution in the nanometer, even subnanometer range to assess morphological features.

\subsubsection{Tracing the conformational change by evaluating ezrin height levels}

We were able to determine absolute height levels of ezrin clusters ranging from $1.5 \mathrm{~nm}$ to $3.5 \mathrm{~nm}$ on DPPC-containing SLBs depending on the activation state of ezrin. Similarly in RIfS experiments, changes in optical thickness in the range of $1.1 \mathrm{~nm}$ to $2.8 \mathrm{~nm}$ were observed due to protein binding. If the refractive index of an adsorbed protein layer is assumed to be $n=1.5,{ }^{[127]}$ the physical layer thickness amounts to $\sim 0.7 \mathrm{~nm}$ to $1.9 \mathrm{~nm}$. Obviously, incomplete protein surface coverage impairs the determination of absolute height levels by RIfS. Nevertheless, it offers a qualitative measure for possible changes in height due to the activation of ezrin by $\mathrm{PIP}_{2}$ binding and pseudophosphorylation.

To evaluate the range of the determined values, it is useful to establish a relation to structural data. So far, no crystal structure of full length ezrin has been published. However, Li et al. succeeded in crystallizing moesin, isolated from Spodoptera frugiperda cells (figure $5.2 \mathrm{~B}$ ). ${ }^{\left[{ }^{156]}\right.}$ With regard to ezrin, only the $N$-terminal FERM domain, also referred to as N-ERMAD, has been reported. ${ }^{[154]}$ Figure 5.21 shows the crystal structure of ezrin N-ERMAD. The indicated $\mathrm{PIP}_{2}$ binding site was derived from the $\mathrm{IP}_{3} /$ radixin-FERM complex crystallized by Hamada et al., ${ }^{[153]}$ while the binding site of DOGS-NTA-Ni, i.e. the $\mathrm{His}_{6}$-tag, is located at the $\mathrm{N}$-terminus. JmolApplet ${ }^{[171]}$ was used to estimate the dimensions of the ezrin N-ERMAD. Assuming an up-right arrangement on the membrane surface, a distance of $\sim 4.7 \mathrm{~nm}$ can be found (figure 5.21 A). This assumption is supported by Blin et al. suggesting a molecularly flat surface in the region of the $\mathrm{PIP}_{2}$ binding site based on the $\mathrm{IP}_{3}$ /radixin-FERM complex and site-directed mutagenesis identifying $\mathrm{PIP}_{2}$ binding sites in ezrin. ${ }^{[119,153,160]}$ Consequently, the measured protein height levels should be approximately $4.7 \mathrm{~nm}$. In addition, $\alpha$-helical linker and $C$-terminal domain are contributing to the total height level. Conclusively, the determined absolute height levels for ezrin clusters on SLBs lie in principal in a reasonable range in comparison with the crystal structure. The slightly lower values can be attributed to the mechanical deformation caused by the cantilever tip during AFM imaging. ${ }^{\left[{ }^{[72]}\right.}$ To minimize this influence, as low set point forces as possible were applied. Moreover, structural deviations might exist between the solid state of ezrin and its structure in fluids. 


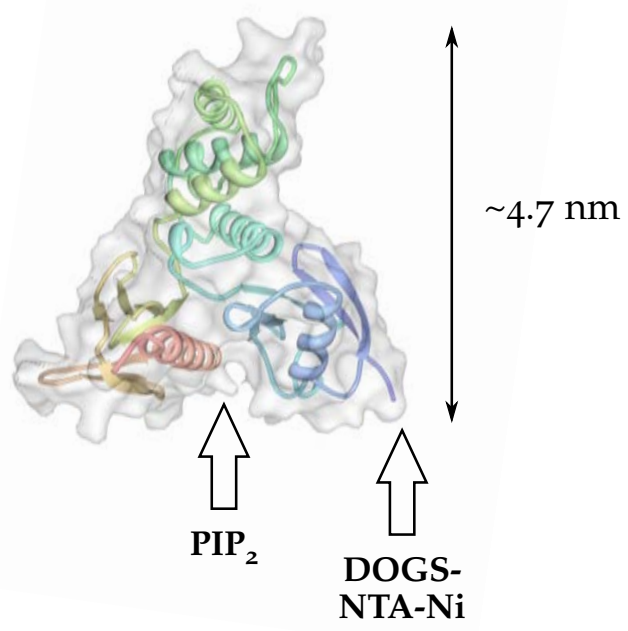

Figure 5.19: Crystal structure of N-ERMAD of ezrin (PDB ID: 1NI2). The assumed binding sites of the receptor lipids PIP $_{2}$ and DOGS-NTA-Ni are indicated by arrows. The PIP binding site was derived from the $\mathrm{IP}_{3}$ /radixin-FERM complex. ${ }^{\left[{ }^{[53]}\right.}$ The His 6 -tag is located at the $N$-terminus.

With regard to the activation process of ezrin, we investigated the individual contributions of either $\mathrm{PIP}_{2}$ or pseudophosphorylation as well as the combination of both. Interestingly, the pseudophosphorylation appears to contribute only to a minor extent on its own to a changed height level as the determined $\triangle O T$ for ezrin $\mathrm{T}_{567} \mathrm{D}$ on DOGS-NTA-Ni-containing SLBs ( $4 \mathrm{~mol} \%$ receptor lipid) is with $(2.0 \pm 0.4) \mathrm{nm}$ rather similar to $(2.3 \pm 0.3) \mathrm{nm}$, which was found for ezrin wildtype under the same conditions in RIfS experiments. This is in agreement with the work of Chambers et al. showing in binding assays that the $T_{5} 67 \mathrm{D}$ mutation in ezrin impairs only to a minor extent the association of $N$ - and C-termini. ${ }^{[173]}$ Jayasundar et al. corroborated this finding by using contrast variation small angular neutron scattering, showing that ezrin wildtype as well as ezrin $\mathrm{T}_{567} \mathrm{D}$ adopt a closed conformation, i.e., no release of the F-actin binding site was detectable upon pseudophosphorylation. ${ }^{[174]}$ However, with regard to an increased F-actin binding capability of ezrin as a result of ezrin activation via pseudophosphorylation, we suggested in a previous study that ezrin $\mathrm{T}_{567} \mathrm{D}$ is able to adopt a partially active conformation. ${ }^{[14]}$ Similarly, Huang et al. could show that the pseudophosphorylation led to an increased cosedimentation of the full length moesin mutant and F-actin, indicating a partial release of the F-actin binding site owing to electrostatic repulsion. ${ }^{\left[{ }^{175]}\right.}$ Considering our results obtained in RIfS measurements, such a partial release of the $C$-terminal region appears to affect the height of ezrin $T_{56}{ }_{7} \mathrm{D}$ aggregates on DOGS-NTA-Ni-containing SLBs only to a minor extent. To further substantiate the RIfS data, it would be of great interest to image ezrin $\mathrm{T}_{5} 67 \mathrm{D}$ im- 
mobilized via the $\mathrm{His}_{6}$-tag by AFM and compare it with the likewise determined height level of $\sim 2.8 \mathrm{~nm}$ for ezrin wildtype on the same membrane system.

Considering the individual contribution of $\mathrm{PIP}_{2}$, we observed a slight decrease in $\triangle O T$ for ezrin wildtype and ezrin $\mathrm{T}_{5} 67 \mathrm{~A}$ bound to $\mathrm{PIP}_{2}$ as compared to the immobilization via DOGS-NTA-Ni. However, this rather subtle change is still within the standard deviation. In case of AFM measurements, we even found an increase in the absolute height level of ezrin wildtype from $(2.8 \pm 1.2) \mathrm{nm}$ on DOGS-NTA-Ni containing SLBs to $(3.5 \pm 0.9) \mathrm{nm}$ for PIP $\mathrm{P}_{2}$ immobilization. Yet the standard deviation in case of the DOGS-NTA-Ni immobilized protein is considerably large, indicating that more data is required to confirm this difference. Moreover, we cannot exclude that the slightly different locations of PIP ${ }_{2}$ and DOGS-NTA-Ni binding site, respectively (figure 5.19), might induce different orientations, thus obscuring an effect of PIP ${ }_{2}$ binding on the observed height level. In general, an effect of PIP $\mathrm{P}_{2}$ binding on the conformational change was expected as Maniti et al. reported a higher sensitivity of ezrin to digestion by chymotrypsin in presence of $\mathrm{PIP}_{2}$, thus concluding that a conformational change renders cleavages sites more accessible. ${ }^{[161]}$ Furthermore, Carvalho et al. examined the changes in the intrinsic tryptophane fluorescence of ezrin wildtype and a mutant deficient in specific $\mathrm{PIP}_{2}$ binding. ${ }^{176]}$ In presence of $\mathrm{PIP}_{2}$ concentrations in the micromolar range, they observed different quenching characteristic, which they attributed to a conformational change in case of the wildtype and to nonspecific interactions for the mutant. Notably, Herrig et al. determined for ezrin wildtype on $\mathrm{PIP}_{2}$-containing POPC-SLBs $(1.8 \pm 0.2) \mathrm{nm}$, while an average height level of $(2.5 \pm 0.2) \mathrm{nm}$ was determined for the same protein bound to DOPC/DOGS-NTA-Ni bilayers. ${ }^{[17]}$ This might be an indication that a correlation of $\mathrm{PIP}_{2}$ induced conformational change and different protein height levels exist. However, with regard to the crystal structure these values are considerably small, indicating that the influence of force during imaging was more pronounced as in our case. Within the context of our results, it appears that the conformational change induced by $\mathrm{PIP}_{2}$ does not necessarily correlate with a change in the protein height level on the membrane surface.

The combination of $\mathrm{PIP}_{2}$ binding and pseudophosphorylation led to a remarkable small $O T$ value and low absolute height level. Ezrin wildtype bound to $\mathrm{PIP}_{2}$ on DPPC-SLBs was characterized by a height level of $(3.5 \pm 0.9) \mathrm{nm}$, while ezrin $\mathrm{T}_{567} \mathrm{D}$ formed protein cluster of $(1.5 \pm 0.8) \mathrm{nm}$ in height. In RIfS experiments with $4 \mathrm{~mol} \%$, the changes in OT of $(2.0 \pm 0.5) \mathrm{nm}$ and $(1.1 \pm 0.4) \mathrm{nm}$ caused by either ezrin wildtype or ezrin $\mathrm{T}_{5} 67 \mathrm{D}$ binding to $\mathrm{PIP}_{2}$ showed the same trend as observed in AFM measurements. This behavior was also found for $2 \mathrm{~mol} \% \mathrm{PIP}_{2}$ content. Considering the distinct height reduction in case of both RIfS and AFM experiments, it appears that in particular the combination of PIP $_{2}$ binding and phosphorylation 
leads to a different protein arrangement on the membrane surface. As it is known that ezrin is able to form dimers and even oligomers, the question arises whether a conformational change of monomeric ezrin at the SLB is sufficient to cause such a reduction or whether the dissociation of membrane bound antiparallel dimers have to be taken into account (figure 5.20). Gel filtration analysis revealed Stokes radii of $4.1 \mathrm{~nm}$ for the monomer and $7.2 \mathrm{~nm}$ for the dimerized form. ${ }^{[177]}$ Moreover, it has been shown that the pseudophosphorylated radixin exists mainly in the monomeric form in solution. ${ }^{[178]}$ However, the same study reported that the dimer peak of ezrin wildtype was only $\sim 30 \%$ of that of the monomeric form. Consequently, the major part of ezrin wildtype should adsorb to the membrane as monomer. This is corroborated by the fact that we observe essentially only one pronounced height level for ezrin wildtype on SLBs. Thus, we conclude that both ezrin wildtype and ezrin $\mathrm{T}_{5} 67 \mathrm{D}$ are mainly bound in their monomeric form to $\mathrm{PIP}_{2}$.

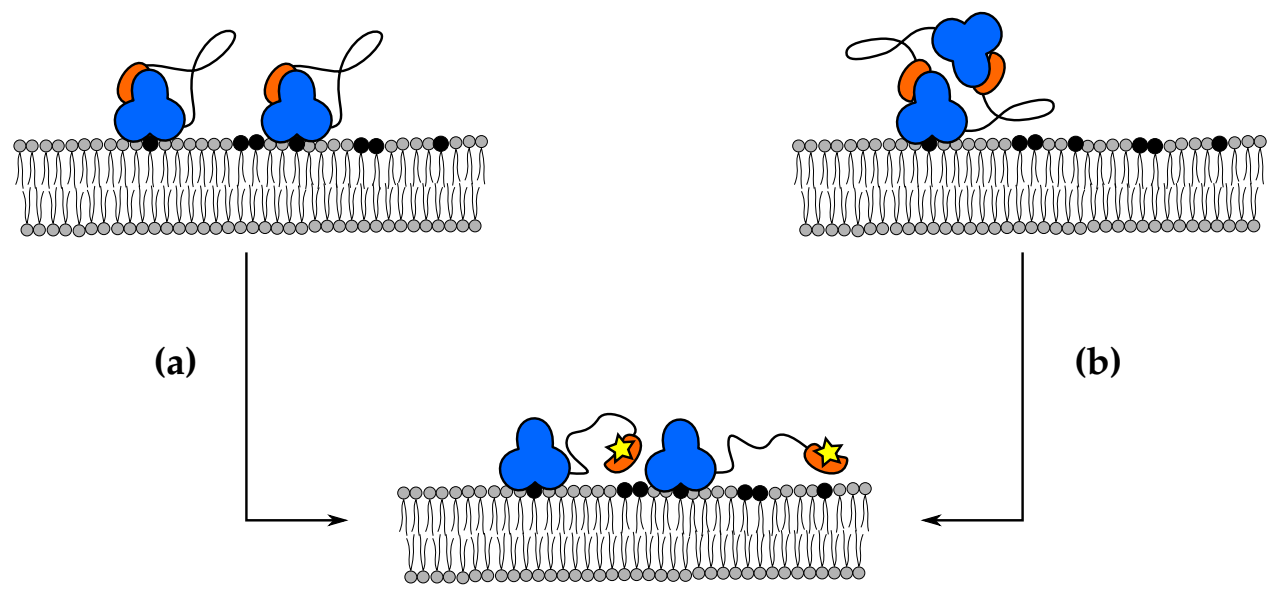

Figure 5.20: Model accounting for different ezrin height levels due to its activation by $\mathrm{PIP}_{2}$ lipids (solid black circles) in combination with phosphorylation (yellow star). Ezrin is composed of N-ERMAD (blue), an $\alpha$-helical linker region (black solid line) and the $C$ terminal part (orange). Phosphorylation triggers release of the C-terminal domain (a) or induces both dissociation of antiparallel dimer and subsequent release of the $C$-terminus (b).

Considering that already the $N$-terminal part of ezrin is characterized by $4.7 \mathrm{~nm}$ in height (figure 5.19), rises the question whether the combination of $\mathrm{PIP}_{2}$ binding and pseudophosphorylation only triggers the release of the $C$-terminal domain or if a different orientation to the membrane is induced. Rotating the crystal structure of N-ERMAD by $90^{\circ}$ reveals a $\sim 2.5 \mathrm{~nm}$ distance (figure 5.21 ), which is much closer to the observed height level. A rather speculative hypothesis is that a profound structural rearrangement might be induced upon the combination of both activation factors $\mathrm{PIP}_{2}$ binding and phosphorylation leading to a different membrane orientation of the $\mathrm{N}$-terminal domain of ezrin. However, a further hint substantiating this idea is the exceptional high protein surface coverage found for ezrin $\mathrm{T}_{567} \mathrm{D}$ bound to $\mathrm{PIP}_{2}$-containing SLBs as compared to ezrin wildtype on the same 
membrane system.

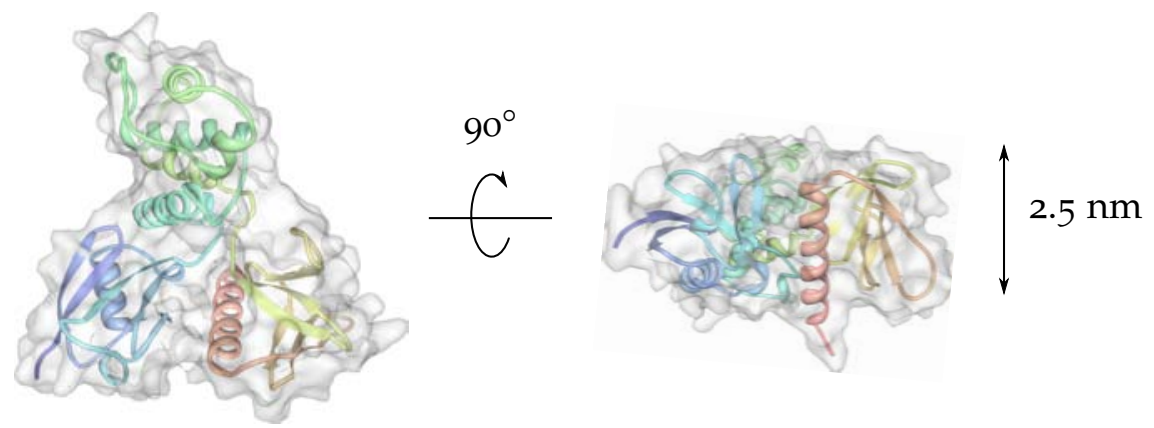

Figure 5.21: Crystal structure of N-ERMAD of ezrin (PDB ID: 1NI2) rotated by $90^{\circ}$.

In conclusion, our results show that the conformational state induced upon full activation, i.e., ezrin $\mathrm{T}_{5} 67 \mathrm{D}$ bound to $\mathrm{PIP}_{2}$, is clearly distinguishable from other conformational states owing to lower height levels as determined by the surface sensitive techniques RIfS and AFM. However, it is worth to note that this does not necessarily exclude a conformational change of ezrin wildtype upon binding to $\mathrm{PIP}_{2}$. Several lines of evidence suggest that $\mathrm{PIP}_{2}$ on its own has indeed an activating influence on ezrin. It rather appears that this conformational state does not correlate with a changed protein height level on SLBs.

\subsubsection{Pre-organization of PIP ${ }_{2}$ influences ezrin cluster morphology}

Based on homogeneous distribution of the fluorescent $\mathrm{PIP}_{2}$ analogue Bodipy TMR$\mathrm{PIP}_{2}$ within POPC- and DPPC-SLBs in CLSM experiments, we assumed for both types of membrane randomly distributed $\mathrm{PIP}_{2}$ and expected similarly shaped ezrin aggregates. However, we observed the formation of an elongated, reticular ezrin arrangement on the DPPC-SLB, whereas rather circularly shaped ezrin aggregates were found in case of the matrix lipid POPC. It appears that the different phases - gel vs fluid phase - cause such deviations. Apparently, the distribution of $\mathrm{PIP}_{2}$ is not completely homogeneous on a scale below the resolution limit of the CLSM. This can most likely be attributed to the fact that the natural PIP $_{2}$ we used is fluid owing to its fatty acid composition. Accordingly, we might have very small fluid $\mathrm{PIP}_{2}$ domains within the gel phase DPPC matrix. Ellenbroek et al. simulated the formation of $\mathrm{PIP}_{2}$ nanoclusters induced by divalent cations. ${ }^{[103]}$ They reported a rather irregularly shape as depicted in figure 5.22 A. 
A

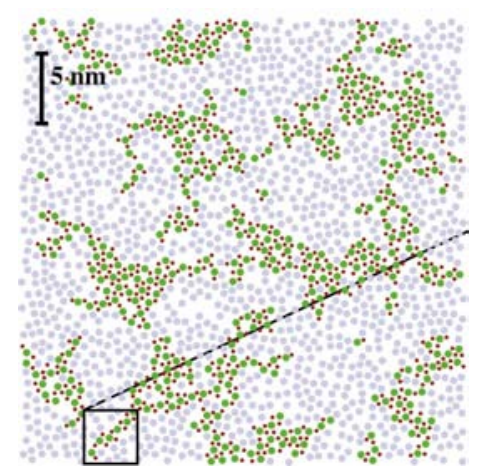

B

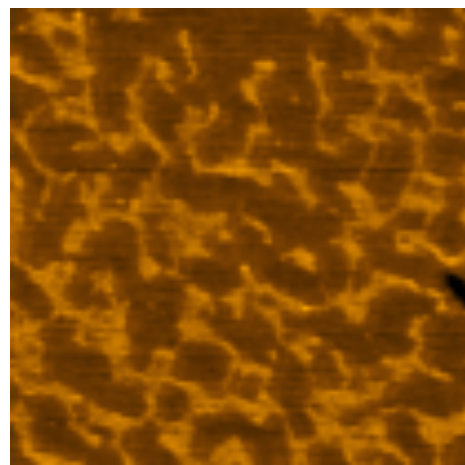

Figure 5.22: A Simulation showing the coarsening of the $\mathrm{PIP}_{2}$ distribution in presence of divalent cations after $3.5 \mathrm{~ns}$. Charged and neutral lipids are dark green and light gray colored, respectively, and divalent ions close to the lipid monolayer are represented by dark red dots. ${ }^{\left[{ }^{103]}\right]}$ B $2 \mu \mathrm{m} \times 2 \mu \mathrm{m}$ section of the AFM height image showing ezrin $\mathrm{T}_{5} 67 \mathrm{D}$ immobilized onto a DPPC/PIP ${ }_{2} /$ Bodipy- ${ }_{12}$-HPC SLB (96.5:3:0.5).

Although no divalent cations were present in our case, it might be that we forced a pre-organization of $\mathrm{PIP}_{2}$ due to nanoscale phase separation. On the one hand, complete mixing within the gel phase formed by DPPC molecules might be unfavorable for $\mathrm{PIP}_{2}$ as it is in the fluid phase. On the other hand, the formation of large, circular $\mathrm{PIP}_{2}$ domains to reduce the line tension is electrostatically impaired since $\mathrm{PIP}_{2}$ has a net negative charge of approximately -3 to -4 under the applied conditions. ${ }^{[88]}$ If we assume that we deal with such a nanoscale aggregation of $\mathrm{PIP}_{2}$, which is impossible to resolve by CLSM, we might have an explanation for the irregularly shaped protein arrangement on PIP $_{2}$-containing DPPC-SLBs (5.22 B). The assumption that ezrin occupies an area of $\sim 3000 \AA^{2}$, while the $\mathrm{PIP}_{2}$ head group covers $\sim 70 \AA^{2}$, gives an explanation for the different order of magnitude of $\mathrm{PIP}_{2}$ nanoclusters and ezrin aggregates in figure 5.22. ${ }^{[119,179]}$ To substantiate this hypothesis, it would be of great interest to investigate the arrangement of ezrin on a DPPC-SLB containing PIP 2 with saturated fatty acyl chains. In this case, we would expect randomly distributed ezrin aggregates forming more circular domains, similar to the situation with POPC assuming that the diffusion has no or only a minor influence. Moreover, high resolution AFM images, e.g. $100 \mathrm{~nm} \times 100 \mathrm{~nm}$ of DPPC-SLBs with varying $\mathrm{PIP}_{2}$ content might reveal such small $\mathrm{PIP}_{2}$ domains.

\subsubsection{Drawing conclusions from the degree of protein surface coverage}

We investigated the degree in protein surface coverage as a function of increasing the $\mathrm{PIP}_{2}$ content within DPPC-SLBs. Herrig et al. reported a completely ezrin covered POPC $/ \mathrm{PIP}_{2}$ (90:10) SLB by AFM, corresponding to $70 \%$ surface coverage. ${ }^{[117]}$ In quartz-crystal microbalance (QCM) experiments, we showed that a $\mathrm{PIP}_{2}$ content of $\sim 7 \mathrm{~mol} \%$ is sufficient to achieve maximum protein coverage of solid-supported 
membranes. ${ }^{\left[{ }^{14]}\right.}$ In this work, we observed a protein surface coverage ranging from $40 \%$ to $57 \%$ with $7 \mathrm{~mol} \% \mathrm{PIP}_{2}$. The lower degree of coverage could be attributed to the fact that DPPC-SLBs contain more membrane defects than POPC-SLBs. Consequently, a systematic underestimation of the protein covered surface was obtained in our case. Notably, Herrig et al. could show by QCM and AFM imaging that the adsorption of ezrin to $\mathrm{PIP}_{2}$-containing membranes is reversible to a certain degree. ${ }^{[117]}$ They suggested the existence of ezrin populations with different affinities, i.e., weakly adsorbed proteins readily desorb upon rinsing with buffer, while larger protein aggregates, formed due to lateral protein-protein interactions, remain irreversibly bound.

A positive effect of $\mathrm{PIP}_{2}$ on the formation of lateral protein-protein interactions might be concluded from the significantly higher protein surface coverage obtained for ezrin wildtype bound via $\mathrm{PIP}_{2}$ to DPPC-SLBs as compared to ezrin wildtype adsorbed to the same receptor lipid content of DOGS-NTA-Ni within DPPC-SLBs. However, recent experiments in our group have shown that the binding of ezrin to DOGS-NTA-Ni-containing membranes is hampered when buffer at low $\mathrm{pH}$ (citrate buffer) was used for spreading. On the one hand, protonation of the carboxylic acid groups at low $\mathrm{pH}$ is expected to reduce the NTA-Ni stability. On the other hand, the buffering agent citrate can compete for $\mathrm{Ni}^{2+}$ with the NTA groups. Although citrate exhibits a lower complex stability with $\mathrm{Ni}^{2+}$ than NTA, we most certainly reduced the number of available NTA-Ni binding sites by using citrate buffer for membrane formation. ${ }^{[180]}$ For this reason, the lower protein surface coverage for DOGS-NTANi-doped membranes can be attributed to a certain degree to the smaller number of available binding sites. Further measurements are required to clarify whether $\mathrm{PIP}_{2}$ might induce lateral protein-protein interactions leading to a higher ezrin surface coverage as compared to DOGS-NTA-Ni-containing membranes.

The comparison of protein surface coverages obtained for ezrin wildtype adsorbed to either POPC- or DPPC-SLBs with same PIP ${ }_{2}$ fractions showed that in case of the POPC matrix, a significantly lower portion of the membrane is covered with protein. We propose that the possible pre-organization of $\mathrm{PIP}_{2}$ we discussed before might account for this observation. Due to the DPPC gel phase matrix, a local enrichment of $\mathrm{PIP}_{2}$ lipids might be induced. As lateral protein-protein interactions appear to be important with regard to the formation of stable protein aggregates, the pre-organization of $\mathrm{PIP}_{2}$ might facilitate such cooperative protein adsorption. In contrast, a completely random distribution of $\mathrm{PIP}_{2}$ is expected within the POPC matrix. The lateral diffusion of $\mathrm{PIP}_{2}$ bound ezrin is decisive for the formation of lateral protein-protein interactions. In case the diffusion of this complex is drastically reduced, the bound ezrin might have already desorbed before the complex encounters another one. However, it has been shown that the lateral mobility of 
lipid bound peripheral proteins can be as high as the one of the respective lipid. ${ }^{[181]}$ In case of POPC $/ \mathrm{PIP}_{2}$-SLBs on silicon support, we determined a diffusion coefficient of $\sim 3 \mu^{2} / \mathrm{s}$ (chapter 4 ). Considering that the AFM tip ( $8 \mathrm{~nm}$ radius) covers $\sim 0.06 \mathrm{\mu m}^{2} / \mathrm{s}$ under our imaging conditions, it would be almost impossible to capture fast diffusing single ezrin-PIP ${ }_{2}$ complexes. This problem inherent to the low temporal resolution of the AFM technique is well-known. ${ }^{[182]}$ Approaches to overcome such issues led to the development of high-speed AFM. ${ }^{[183,184]}$

Notably, we observed an increase in surface coverage for ezrin $\mathrm{T}_{5} 67 \mathrm{D}$ on $\mathrm{PIP}_{2^{-}}$ containing DPPC-SLBs as compared to ezrin wildtype under the same conditions. In the light of full activation leading to the dissociation of $\mathrm{N}$-terminal and $\mathrm{C}$ terminal domains, a more extended structure of ezrin $\mathrm{T}_{5} 67 \mathrm{D}$ appears reasonable. Accordingly, fully activated ezrin, i.e., in our case ezrin $\mathrm{T}_{567} \mathrm{D}$ immobilized via $\mathrm{PIP}_{2}$, requires more surface area than ezrin wildtype. Moreover, it would be of great interest to examine the protein arrangement of ezrin $\mathrm{T}_{5} 67 \mathrm{D}$ on $\mathrm{PIP}_{2}$ containing POPC-SLBs.

\subsection{CONCLUSION}

In this work, we investigated the consequences of ezrin activation by the surfacesensitive techniques RIfS and AFM with regard to height levels of ezrin monolayers and their surface coverage on solid-supported membranes. In particular, we addressed the individual steps involved in ezrin activation, i.e., PIP $_{2}$ binding and phosphorylation by using ezrin wildtype and nonphosphorylatable as well as pseudophosphorylated variants in combination with different immobilization strategies.

We were able to show that the pseudophosphorylation on its own does not substantially contribute to a changed height level of protein clusters.

With regard to the individual contribution of $\mathrm{PIP}_{2}$, we could neither detect a substantial decrease or increase in height in presence of $\mathrm{PIP}_{2}$, indicating that this influence cannot be resolved unambiguously by evaluating the height level alone. Considering that the activating influence has been shown in other studies, ${ }^{[14,15]}$ the height level analysis as measure for ezrin activation is not sufficient. Partial activation via $\mathrm{PIP}_{2}$ might favor lateral protein-protein interaction leading to higher protein surface coverage. Consequently, activation of ezrin might be not only restricted to a dissociation of $\mathrm{N}$ - and $\mathrm{C}$-terminal domains leading to lower protein height levels, but also include the formation of a dense protein layer to offer as many binding site as possible toward F-actin.

The combination of $\mathrm{PIP}_{2}$ binding and pseudophosphorylation led to a clearly lower protein height level and to an increased protein surface coverage. Conse- 
quently, we conclude that the full activation of ezrin causes a profound structural rearrangement on the membrane surface including dissociation of the N-ERMAD/CERMAD complex and lateral protein-protein interactions.

Remarkable was the effect of the gel phase DPPC matrix as it apparently forced a local enrichment of $\mathrm{PIP}_{2}$ on the nanoscale facilitating cooperative ezrin adsorption. Consequently, this model system offers the possibility to generate locally enriched $\mathrm{PIP}_{2}$ areas without the need of proteins or divalent cations promoting $\mathrm{PIP}_{2}$ sequestration. This system may serve as model for the investigation of proteins, which require a comparably high local concentration of $\mathrm{PIP}_{2}$, in a well-defined environment.

\subsection{CO-WORKERS}

Kramer, Corinna; Gerdes, Benjamin; Mey, Ingo; Wechsler, Cindy

\subsection{EXPERIMENTAL DETAILS}

MATERIALS The materials used for transformation, plasmid isolation, and protein purification are given in sections 3.1.1, 3.1.2, and 3.1.4. The lipids 1,2-dioleoyl-sn-glycero-3-phosphocholine (DOPC), 1,2-dipalmitoyl-sn-glycero-3-phosphocholine (DPPC), 1-palmitoyl-2-oleoyl-sn-glycero-3-phosphocholine (POPC), L- $\alpha$-phosphatidylinositol-4,5-bisphosphate (PIP $\left.{ }_{2}\right)$, and 1,2-Dioleoyl-sn-glycero-3-[( $N$ - $(5$-amino-1-carboxypentyl) iminodiacetic acid)succinyl] (nickel salt) (DOGS-NTA-Ni) were obtained from Avanti polar lipids (Alabaster, USA). The fluorophor $\beta$-BODIPY ${ }^{\circledR} 500 /$ $510 \mathrm{C}_{12}$-HPC (2-(4,4-difluoro-5-methyl-4-bora-3a,4a-diaza-s-indacene-3-dodecanoyl)1-hexadecanoyl-sn-glycero-3-phosphocholine, Bodipy- $\mathrm{C}_{12}-\mathrm{HPC}$ ) was obtained from Life Technologies (Darmstadt, Germany). Silicon wafers with a $5 \mu \mathrm{m}$ layer of silicon dioxide were used as RIfS transducer chips (ABC - Active business company, Munich, Germany). Silicon wafers for AFM measurements ( $100 \mathrm{~nm} \mathrm{SiO}_{2}$ layer) were purchased from Silicon materials (Kaufering, Germany). All chemicals were of the highest purity available. Water was purified first through a Millipore water purification system Milli-RO 3 plus and finally with a Millipore ultrapure water system Milli-Q plus 185 (specific resistance $=18.2 \mathrm{M} \Omega / \mathrm{cm}$ ) (Billerica, USA).

RIFS MEASUREMENT The experimental setup used is described in more detail in section 3.3.3.1. SUVs composed of POPC/PIP ${ }_{2}$ or DOPC/DOGS-NTA-Ni were prepared according to section 3.2.2.2 in E1 buffer. A hydrophilized RIfS wafer was mounted into the flow cell.and a baseline was recorded by flowing E1 buffer through the system. The spreading process was performed under closed-loop con- 
ditions and monitored by RIfS as an increase in $\triangle O T$ to $\sim 6 \mathrm{~nm}$. After bilayer formation, the SLB was rinsed copiously with E1 buffer to remove excess vesicles. Protein binding (final concentration $\sim 0.7 \mu \mathrm{M}$ ) generated further increase in $\triangle O T$. To determine the amount of specifically adsorbed protein, an additional rinsing step with EI buffer was performed. The effective $\triangle O T$ was extracted from the difference between the final $\triangle O T$ level and the plateau immediately before protein addition.

AFM IMAGING SLBs were prepared on silicon support according to the procedure described in section 3.2.2.3. SUVs were prepared from lipid films composed of either DPPC/PIP 2 /Bodipy-C ${ }_{12}-\mathrm{HPC}$, DPPC/DOGS-NTA-Ni/Bodipy-C $\mathrm{C}_{12}{ }^{-}$ $\mathrm{HPC}$, or $\mathrm{POPC} / \mathrm{PIP}_{2} /$ Bodipy- $\mathrm{C}_{12}-\mathrm{HPC}$ with $\mathrm{PIP}_{2}$ fractions ranging from $3 \mathrm{~mol} \%$ to $7 \mathrm{~mol} \%$ in buffer $\mathrm{C}\left(20 \mathrm{~mm}\right.$ Citrate, $50 \mathrm{~mm} \mathrm{KCl}$, o.1 mM EDTA, o.1 $\mathrm{mm} \mathrm{NaN}_{3}$, $\mathrm{pH} 4.8$ ). Bodipy- $\mathrm{C}_{12}-\mathrm{HPC}$ was added at $0.5 \mathrm{~mol} \%$ to validate bilayer formation by fluorescence spectroscopy before the AFM measurement. Each SLB was imaged in tapping mode before protein addition in E1 buffer to evaluate the bilayer quality with regard to defects and adhered excess vesicles. Only membranes characterized by minor adhesion of vesicles were used for protein binding. A final concentration of $\sim 0.6 \mu \mathrm{M}$ ezrin in E1 buffer was employed and the sample was incubated at $4{ }^{\circ} \mathrm{C}$ overnight. Before the measurement the sample was rinsed with E1 buffer to remove nonspecifically bound protein and thermally equilibrated for $45 \mathrm{~min}$. CSC 37 cantilevers with a nominal spring constant of $0.65 \mathrm{pN} / \mathrm{nm}$ were purchased from MikroMasch (Wetzlar, Germany). Imaging of protein-covered SLBs was performed in tapping mode applying low forces by using the NanoWizard 3 (JPK Instruments, Berlin, Germany). 10 $\mu \mathrm{m} \times 10 \mu \mathrm{m}$ images were recorded with a line scan rate ranging from $0.4 \mathrm{~Hz}$ to $0.6 \mathrm{~Hz}$. The freeware Gwyddion was used for further data processing. Histogram height analysis was carried out using the histogram height analysis tool based in MATLAB. 
$5 \cdot 7$ APPENDIX

DNA sequence of ezrin wildtype with fused $N$-terminal $\mathrm{His}_{6}$-tag.

1 ATGGGCAGCA GCCATCATCA TCATCATCAC AGCAGCGGCC TGGTGCCGCG CGGCAGCCAT

61 ATGGCTAGCA TGACTGGTGG ACAGCAAATG GGTCGCGGAT CCGAATTCAT GCCGAAACCA 121 ATCAATGTCC GAGTTACCAC CATGGATGCA GAGCTGGAGT TTGCAATCCA GCCAAATACA 181 ACTGGAAAAC AGCTTTTTGA TCAGGTGGTA AAGACTATCG GCCTCCGGGA AGTGTGGTAC 241 TTTGGCCTCC ACTATGTGGA TAATAAAGGA TTTCCTACCT GGCTGAAGCT GGATAAGAAG 301 GTGTCTGCCC AGGAGGTCAG GAAGGAGAAT CCCCTCCAGT TCAAGTTCCG GGCCAAGTTC 361 TACCCTGAAG ATGTGGCTGA GGAGCTCATC CAGGACATCA CCCAGAAACT TTTCTTCCTC 421 CAAGTGAAGG AAGGAATCCT TAGCGATGAG ATCTACTGCC CCCCTGAGAC TGCCGTGCTC 481 TTGGGGTCCT ACGCTGTGCA GGCCAAGTTT GGGGACTACA ACAAAGAAGT GCACAAGTCT 541 GGGTACCTCA GCTCTGAGCG GCTGATCCCT CAAAGAGTGA TGGACCAGCA CAAACTTACC 601 AGGGACCAGT GGGAGGACCG GATCCAGGTG TGGCATGCGG AACACCGTGG GATGCTCAAA 661 GATAATGCTA TGTTGGAATA CCTGAAGATT GCTCAGGACC TGGAAATGTA TGGAATCAAC 721 TATTTCGAGA TAAAAAACAA GAAAGGAACA GACCTTTGGC TTGGAGTTGA TGCCCTTGGA 781 CTGAATATTT ATGAGAAAGA TGATAAGTTA ACCCCAAAGA TTGGCTTTCC TTGGAGTGAA 841 ATCAGGAACA TCTCTTTCAA TGACAAAAAG TTTGTCATTA AACCCATCGA CAAGAAGGCA 901 CCTGACTTTG TGTTTTATGC CCCACGTCTG AGAATCAACA AGCGGATCCT GCAGCTCTGC 961 ATGGGCAACC ATGAGTTGTA TATGCGCCGC AGGAAGCCTG ACACCATCGA GGTGCAGCAG 1021 ATGAAGGCCC AGGCCCGGGA GGAGAAGCAT CAGAAGCAGC TGGAGCGGCA ACAGCTGGAA 1081 ACAGAGAAGA AAAGGAGAGA AACCGTGGAG AGAGAGAAAG AGCAGATGAT GCGCGAGAAG 1141 GAGGAGTTGA TGCTGCGGCT GCAGGACTAT GAGGAGAAGA CAAAGAAGGC AGAGAGAGAG 1201 CTCTCGGAGC AGATTCAGAG GGCCCTGCAG CTGGAGGAGG AGAGGAAGCG GGCACAGGAG 1261 GAGGCCGAGC GCCTAGAGGC TGACCGTATG GCTGCACTGC GGGCTAAGGA GGAGCTGGAG 1321 AGACAGGCGG TGGATCAGAT AAAGAGCCAG GAGCAGCTGG CTGCGGAGCT TGCAGAATAC 1381 ACTGCCAAGA TTGCCCTCCT GGAAGAGGCG CGGAGGCGCA AGGAGGATGA AGTTGAAGAG 1441 TGGCAGCACA GGGCCAAAGA AGCCCAGGAT GACCTGGTGA AGACCAAGGA GGAGCTGCAC 1501 CTGGTGATGA CAGCACCCCC GCCCCCACCA CCCCCCGTGT ACGAGCCGGT GAGCTACCAT 1561 GTCCAGGAGA GCTTGCAGGA TGAGGGCGCA GAGCCCACGG GCTACAGCGC GGAGCTGTCT 1621 AGTGAGGGCA TCCGGGATGA CCGCAATGAG GAGAAGCGCA TCACTGAGGC AGAGAAGAAC 1681 GAGCGTGTGC AGCGGCAGCT GCTGACGCTG AGCAGCGAGC TGTCCCAGGC CCGAGATGAG 1741 AATAAGAGGA CCCACAATGA CATCATCCAC AACGAGAACA TGAGGCAAGG CCGGGACAAG 1801 TACAAGACGC TGCGGCAGAT CCGGCAGGGC AACACCAAGC AGCGCATCGA CGAGTTCGAG 1861 GCCCTGTAA 
Amino acid sequence of ezrin wildtype with fused $\mathrm{N}$-terminal $\mathrm{His}_{6}$-tag. The threonine highlighted in orange is substituted for an alanine (ezrin $\mathrm{T}_{567} \mathrm{~A}$ ) or an aspartate (ezrin $\mathrm{T}_{567} \mathrm{D}$ ). The position 567 is with respect to the initiator methionine in human ezrin (green).

1 MGSSHHHHHH SSGLVPRGSH MASMTGGQQM GRGSEFMPKP INVRVTTMDA ELEFAIQPNT

61 TGKQLFDQVV KTIGLREVWY FGLHYVDNKG FPTWLKLDKK VSAQEVRKEN PLQFKFRAKF 121 YPEDVAEELI QDITQKLFFL QVKEGILSDE IYCPPETAVL LGSYAVQAKF GDYNKEVHKS 181 GYLSSERLIP QRVMDQHKLT RDQWEDRIQV WHAEHRGMLK DNAMLEYLKI AQDLEMYGIN 241 YFEIKNKKGT DLWLGVDALG LNIYEKDDKL TPKIGFPWSE IRNISFNDKK FVIKPIDKKA 301 PDFVFYAPRL RINKRILQLC MGNHELYMRR RKPDTIEVQQ MKAQAREEKH QKQLERQQLE 361 TEKKRRETVE REKEQMMREK EELMLRLQDY EEKTKKAERE LSEQIQRALQ LEEERKRAQE 421 EAERLEADRM AALRAKEELE RQAVDQIKSQ EQLAAELAEY TAKIALLEEA RRRKEDEVEE 481 WQHRAKEAQD DLVKTKEELH LVMTAPPPPP PPVYEPVSYH VQESLQDEGA EPTGYSAELS 541 SEGIRDDRNE EKRITEAEKN ERVQRQLLTL SSELSQARDE NKRTHNDIIH NENMRQGRDK 601 YKTLRQIRQG NTKQRIDEFE AL* 



\section{A MODEL SYSTEM EXPLORING THE FORCES AT THE MEMBRANE CYTOSKELETON INTERFACE}

\subsection{INTRODUCTION}

\section{The ezrin F-actin connection}

The organization and dynamic characteristics of the cell membrane architecture are regulated at the interface between the membrane and the subjacent cortical actin cytoskeleton. This structural framework is tailored by the cell in response to external and internal stimuli, thus participating in various fundamental cellular processes including adhesion, migration, polarization, division, and differentiation. ${ }^{[1,185,186]}$ The key player within this carefully balanced interplay is filamentous actin (F-actin), a semi-flexible polymer composed of globular actin monomers (G-actin) with a molecular weight of $43 \mathrm{kDa}$ each. F-actin is characterized by a mean diameter of $7 \mathrm{~nm}$ and its variable length ranging from $0.1 \mu \mathrm{m}$ to $20 \mu \mathrm{m}$ (figure 6.1). ${ }^{[187,188]}$ Each monomeric subunit is rotated by $166^{\circ}$, thus appearing morphologically as two righthanded, entwined helices. ${ }^{[189]} \mathrm{X}$-ray analysis revealed a nucleotide binding site occupied either by adenosine triphosphate (ATP) or adenosine diphosphate (ADP) as well as several binding sites for divalent cations such as $\mathrm{Ca}^{2+}$ or $\mathrm{Mg}^{2+}{ }^{2}{ }^{[190]}$

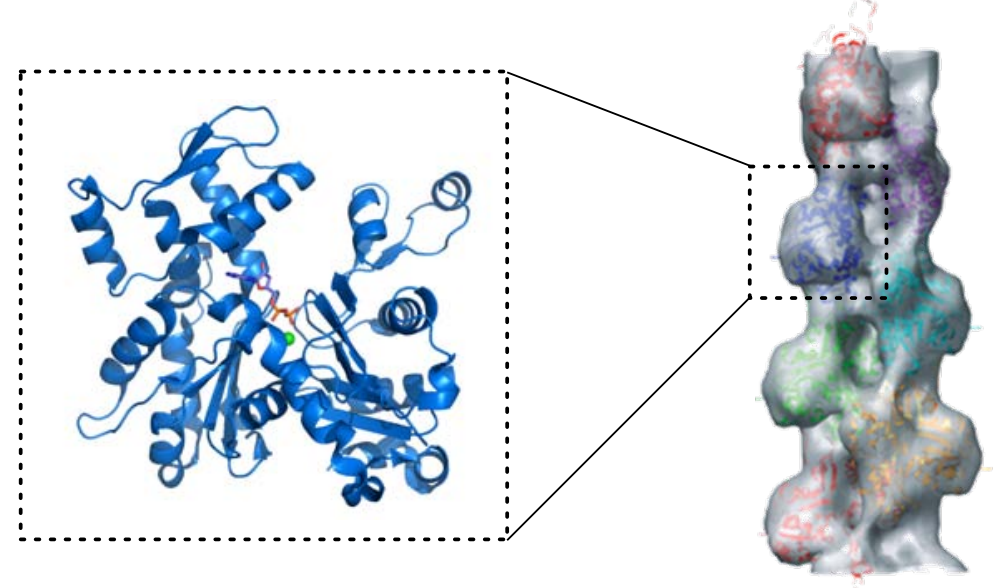

Figure 6.1: Filamentous actin (gray shaded) is composed of globular actin monomers. The monomeric subunit (blue) is complexed with ADP and $\mathrm{Ca}^{2+}$. Adapted from Otterbein and Oda. ${ }^{[190,191]}$

The dynamic and adaptive characteristics of the cortical actin cytoskeleton originate in the fast polymerization and depolymerization of actin. ${ }^{[192,193]}$ Within the cell, these processes are regulated by a vast number of proteins. Despite this com- 
plexity in vivo, the basic polymerization of monomeric actin is easily achieved in vitro under physiological conditions. In particular, the presence of divalent ions and raising the temperature effectively reduces the required critical monomer concentration. The assembly of F-actin encompasses three different phases: (1) nucleation, (2) elongation, and (3) steady-state. The initial phase is characterized by a lag period, where ATP-G-actin oligomerizes to unstable aggregates. Once a length of three to four subunits is reached, the oligomer can act as nucleus, growing rapidly to a filament during the second phase. The incorporated ATP is hydrolyzed to ADP, thus forming ADP-containing subunits. F-actin is intrinsically polarized owing to the oriented head-to-tail assembly of monomers. This polarity is reflected as such that the addition of monomers is preferred at one end, namely the $(+)$ end, as it exhibits a lower critical concentration for elongation than the other one, the (-) end. The growth phase proceeds until equilibrium between monomer and filament is reached, where a steady exchange between monomer and subunits at the ends occurs; however, no more net increase in length is observed. The equilibrium monomer concentration in this so-called treadmilling state is the critical concentration. ${ }^{[194]}$

To translate and direct the forces generated by assembly and disassembly of Factin, a dynamic linkage between plasma membrane and cytoskeleton is required. Among such linker proteins are the members of the ERM (ezrin, radixin, moesin) protein family. By providing a regulated linkage, ERM proteins are implicated to contribute to the organization of structurally and functionally distinct cortical domains, thus participating in adhesion, motility, and fundamental developmental processes. ${ }^{[1,3]}$ Studies have shown that the function of ERMs is conformationally regulated by intramolecular head-to-tail association (figure 6.2). A binding site for F-actin was identified in an $\mathrm{N}$-terminally truncated form of ezrin, whereas no in vitro F-actin association was observed in case of the full length protein. ${ }^{[6,157]}$ Experiments on the association characteristics of truncated ezrin fusion proteins provided further support for the conformational masking model, predicting an apparently dormant, closed conformation due to strongly interacting $N$-terminal and C-terminal ERM association domains (N-ERMAD, C-ERMAD). [7] Thus, an open, active conformation requires the dissociation of the N-ERMAD/C-ERMAD interaction to allow for F-actin binding.

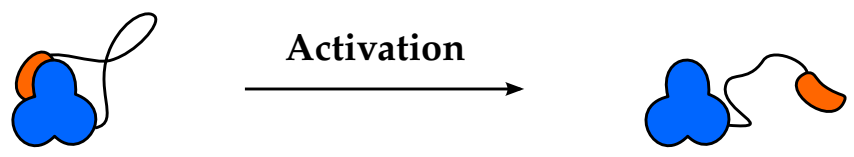

Figure 6.2: Schematic of the conformational masking model. Activation leads to dissociation of N-ERMAD (blue) and C-ERMAD (orange) as such that the closed, dormant state (left) transitions into the open, active conformation (right) capable of binding F-actin. 
After extensive research, two factors have emerged to play a pivotal role in the ERM activation process. A conserved threonine $(\mathrm{T})$ residue within the F-actin binding site was identified for ezrin, radixin, and moesin $-\mathrm{T}_{56} 6, \mathrm{~T}_{5} 64$, and $\mathrm{T}_{55} 8$, respectively - as target for phosphorylation by Rho-kinase and protein kinase $C \Theta \Theta^{[8,9]}$ Furthermore, cosedimentation revealed that a binding site specific to the phosphoinositide $\mathrm{L}$ - $\alpha$-phosphatidylinositol-4,5-bisphosphate $\left(\mathrm{PIP}_{2}\right)$ is located within the $N$ terminal part of ezrin. ${ }^{[10]}$ Based on these findings, a two-step model was proposed for ERM activation comprising recruitment to the membrane by $\mathrm{PIP}_{2}$ and subsequent phosphorylation of the conserved threonine residue in the $C$-terminal domain. ${ }^{[12,13]}$ However, the extent of contribution to the activation regarding the individual activating factor is still subject of controversial discussion. No differences in F-actin binding capability of phosphorylated and non-phosphorylated C-terminal radixin were detected. ${ }^{[9]}$ A similar behavior was observed by Huang et al., mimicking the phosphorylation of moesin by substitution of $\mathrm{T}_{55} 8$ with aspartate (T558D). However, they could show that the pseudophosphorylation enhanced significantly the F-actin binding capability of the full length moesin mutant in cosedimentation assays, indicating a partial release of the F-actin binding site owing to electrostatic repulsion. ${ }^{[175]}$ The pivotal role of $\mathrm{PIP}_{2}$ as activation factor has emerged primarily in in vivo studies. Fievet et al. could show that, although the $\mathrm{T}_{567} \mathrm{D}$ mutation in ezrin circumvents the requirement for $\mathrm{PIP}_{2}$ binding for the release of masked binding sites, it is still essential with regard to the correct apical localization of ezrin. ${ }^{[12]}$ Moreover, Yonemura et al. suggested that the specific phosphorylation is rather required for stabilizing activated ERMs than for their activation. ${ }^{[11]}$ In a recent confocal laser scanning microscopy (CLSM) based assay to probe the F-actin binding capability of ezrin, we were able to show a synergism between phosphorylation and $\mathrm{PIP}_{2}{ }^{\left[{ }^{14]}\right]}$ Furthermore, colloidal probe experiments investigating the influence of $\mathrm{PIP}_{2}$ binding on the interaction between ezrin and F-actin revealed a distinct increase in adhesion forces and cumulative adhesion energy in presence of $\mathrm{PIP}_{2} \cdot{ }^{[15]}$

\section{Bonds under load - Force induced unbinding}

The F-actin cytoskeleton is the major force bearing structure within the cell, providing mechanical stability and sustaining forces. Evidently, the bond between F-actin and ezrin is exposed to force as well. Therefore, we regard the atomic force microscope (AFM) as the method of choice to investigate the behavior of this interaction under force load as it is able to resolve forces in the range of piconewtons. ${ }^{[195]}$ In the framework of Kramers' rate theory, the dissociation of a formed bond to an unbound state can be described as thermally activated escape over an energetic barrier, also referred to as transition state. ${ }^{[196,197]}$ A schematic representation of the 
energy landscape or binding potential describing a dissociating ligand-receptor interaction along the reaction coordinate $x$ is depicted in figure 6.3 A. The bound state is characterized by an energetic minimum whose separation to the transition state along the reaction coordinate is characterized by $x^{\ddagger}$, also referred to as potential width. The rate at which dissociation occurs is given by $k_{\text {off }}^{0}$.

A

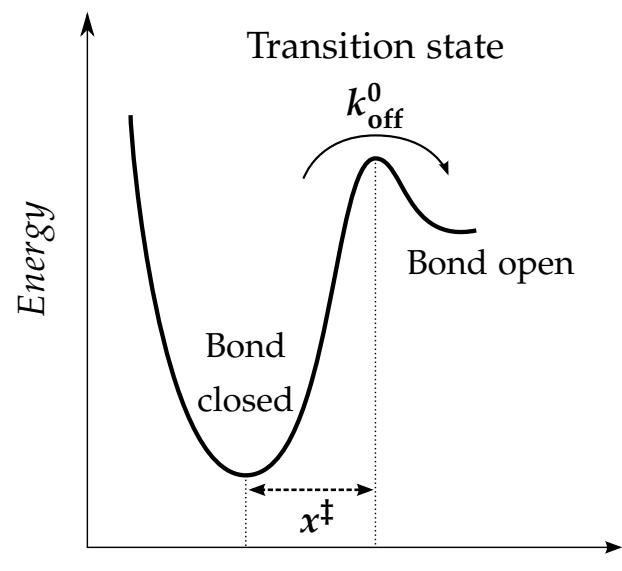

Reaction coordinate
B

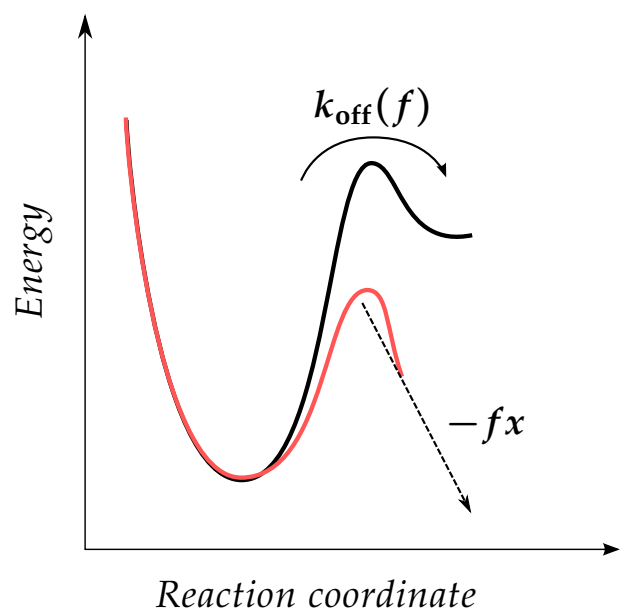

Figure 6.3: A Schematic representation of the energy landscape for the dissociation process of a receptor-ligand interaction in absence of force load. The transition state barrier separates bound (bond closed) and unbound (bond open) state. Its separation to the bound state is characterized by $x^{\ddagger}$, while the rate at which dissociation occurs is given by $k_{\text {off }}^{0}$. B The application of an external force $f$ tilts the energy landscape (red line) and lowers the energetic barrier. Accordingly, the dissociation rate $k_{\text {off }}$ is a function of the applied force load $f$.

The first model considering the influence of force load on bond dissociation was derived by Bell in 1978. ${ }^{[198]}$ Following Zhurkov's kinetic concept of the strength of solids, ${ }^{[199]}$ he introduced the unbinding rate constant $k_{\text {off }}$ :

$$
k_{\text {off }}=k_{\text {off }}^{0} \exp \left[\beta f x^{\ddagger}\right] \quad \text { with } \quad \beta=\frac{1}{k_{\mathrm{B}} T},
$$

where $k_{\text {off }}^{0}$ denotes the unbinding rate at zero force and $f$ is the applied force per bond, acting along the reaction coordinate $x$. The potential width $x^{\ddagger}$ is assumed to be independent of the applied force. $T$ is the temperature in Kelvin and $k_{\mathrm{B}}$ the Boltzmann's constant. The loading rate $v_{F}$, i.e., the force per time interval a bond is loaded, is critical with regard to the distinction between near-equilibrium and non-equilibrium. The near-equilibrium regime is characterized by slow loading rates where rebinding is a relevant process, whereas in the non-equilibrium regime fast loading rates lead to complete suppression of rebinding events. In case of force spectroscopic measurements, non-equilibrium is in particular prevalent as bonds are generally probed fast with a comparably soft cantilever. Moreover, the experiments are usually performed at a constant pulling speed, i.e., the force load on the bond changes over time (force ramp). The energetic landscape is tilted by the 
applied force, while the energetic barrier is lowered (figure 6.3 B). Assuming that the unbound state is located far away from the energy barrier, i.e., the cantilever is sufficiently soft, the cantilever potential can be approximated as linear function. By using the Bell model, Evans and Ritchie derived a kinetic approach to bond rupture occurring in the non-equilibrium based on Kramers' rate theory: ${ }^{\text {[200,201] }}$

$$
F^{*}=\frac{1}{\beta x^{\ddagger}} \ln \left[\frac{\beta x^{\ddagger}}{k_{\text {off }}^{0}}\right]+\frac{1}{\beta x^{\ddagger}} \ln \left[v_{F}\right] .
$$

$F^{*}$ denotes the most probable rupture force, while $v_{F}$ is the loading rate. Equation 6.2 reveals a linear dependence of $F^{*}$ on the logarithm of $v_{F}$. This appealingly simple relationship is used throughout literature to extract $x^{\ddagger}$ and $k_{\text {off }}^{0}$ by linear fitting. ${ }^{[202,203]}$ The slope is used to calculate $x^{\ddagger}$, while the ordinate intercept yields $k_{\text {off }}^{0}$. These values are basic parameters describing the energy profile along the force-driven reaction coordinate. The concept of applying a wide range of loading rates to investigate bond strength is also referred to as dynamic or molecular force spectroscopy (MFS). It has been used to explore energy landscapes of the domain unfolding in titin, ${ }^{[40]}$ the biotin/(strept)avidin interaction, ${ }^{[204]}$ the separation of complementary DNA-strands, ${ }^{[205]}$ and the interaction between cadherin dimers. ${ }^{[206]}$

The poorly defined apex geometry in case of a conventional AFM tip led to the development of the colloidal probe microscopy (CPM). This technique was established by Ducker and Butt to measure surface forces. ${ }^{[207,208]}$ They modified a cantilever by attaching a spherical particle at its end. The well-defined contact geometry between sphere and flat surface allows a more quantitative determination of surface forces by using contact theories such as the Hertz model. ${ }^{[209]}$ Due to its favorable geometry in terms of low curvature as compared to a conventional AFM tip, this technique has been used for the investigation of membrane-membrane interactions. ${ }^{[210]}$ Moreover, F-actin was attached to a colloidal probe and its interaction with ezrin bound to $\mathrm{PIP}_{2}$ was investigated, which was the starting point of the work presented in this chapter. ${ }^{[15]}$

We aimed to elucidate the interaction between F-actin and ezrin by means of AFM. On the one hand, we employed colloidal probe microscopy (CPM), mimicking the situation in microvilli where many ezrin molecules are present to bind tightly to F-actin. We analyzed adhesion forces and surface adhesion energies as a function of ezrin activation. On the other hand, we made use of single molecule force measurements (SMFM) investigating the interaction between a single ezrin and F-actin for comparison with CPM experiments. Moreover, molecular force spectroscopy (MFS) allowed us to determine kinetic parameters, namely $k_{\text {off }}^{0}$ and $x^{\ddagger}$, describing 
the bond between a single ezrin and F-actin, thus deriving basic information about the energy landscape.

In particular, we addressed the question how the interaction is influenced by the degree of ezrin activation with regard to its F-actin binding capability. To map the influence of the individual activation step, ezrin wildtype and two mutants, ezrin $\mathrm{T}_{56} 6 \mathrm{~A}$ and ezrin $\mathrm{T}_{567} \mathrm{D}$, were used. In the latter one, the threonine at position 567 was substituted for an aspartate, thus mimicking a permanent phosphorylated form. Due to the replacement of threonine by alanine, ezrin T567A is considered constitutively inactive with regard to phosphorylation and therefore used in cell experiments. ${ }^{\left[{ }^{162-164]}\right.}$ Moreover, we employed a submicellar PIP ${ }_{2}$ concentration to induce ezrin activation. This strategy has been successfully used by other groups. ${ }^{[176,211,212]}$

\subsection{RESULTS}

\subsubsection{Surface functionalization strategy and setup}

The schematic drawing in figure 6.4 shows the basic experimental setup used in force measurements and spectroscopy, which is characterized by an ezrin coated cantilever tip and an F-actin covered surface.

A $6 \times$ histidine $\left(\mathrm{His}_{6}\right)$ tag was fused to the $\mathrm{N}$-terminus of ezrin to allow on the one hand its specific purification by using nickel nitrilotriacetic acid (NTA-Ni) bearing agarose. On the other hand, this tag provides, together with a NTA-Ni terminated self-assembled monolayer (SAM) on gold containing cantilevers, both uniform orientation and specific binding of the protein. The SAM was composed of $\mathrm{N}-\left[5-\left[\left[\left[{ }^{20}-\right.\right.\right.\right.$ mercapto-3,6,9-trioxaeicos-1-yl)oxo]carbonyl]amino]-1-carboxypentyl]iminodiacetic acid (NTA thiol) and (1-mercaptoundec-11-yl)tri(ethylene glycol), where the latter one is the matrix thiol to reduce the protein density on the surface, prevent nonspecific binding and denaturation of proteins. The position of the $\mathrm{His}_{6}$-tag was chosen in such a way to mimic the $N$-terminal membrane attachment of ezrin and to prevent blocking of the F-actin binding site, which is located in the C-terminus. In molecular force spectroscopy or single molecule force measurements (MFS/SMFM, figure $6.4 \mathrm{~A}$ ), the protein concentration at the tip is diluted such that in the ideal case only one protein is able to bind to F-actin. On the contrary, colloidal probe microscopy (CPM, figure 6.4 B) provides a well defined contact area, where many proteins are located.

F-actin was attached via electrostatic interaction to a positively charged SAM, which is composed of (11-mercaptoundecyl)trimethylammonium (AUT ${ }^{+}$) and adsorbed onto a gold coated silicon wafer. We refrained from attaching F-actin to the 
cantilever with regard to SMFM and MFS experiments as the attachment of a single rod-like macromolecule to a sharp cantilever tip appears rather challenging.

A

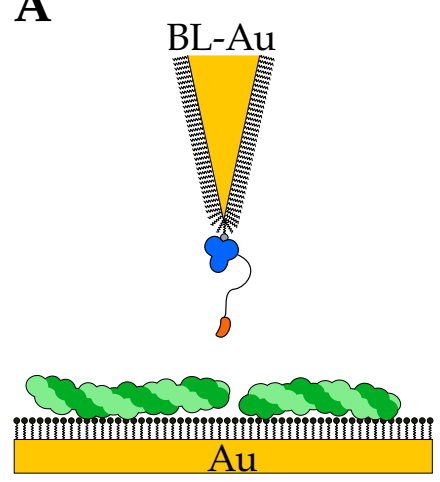

B

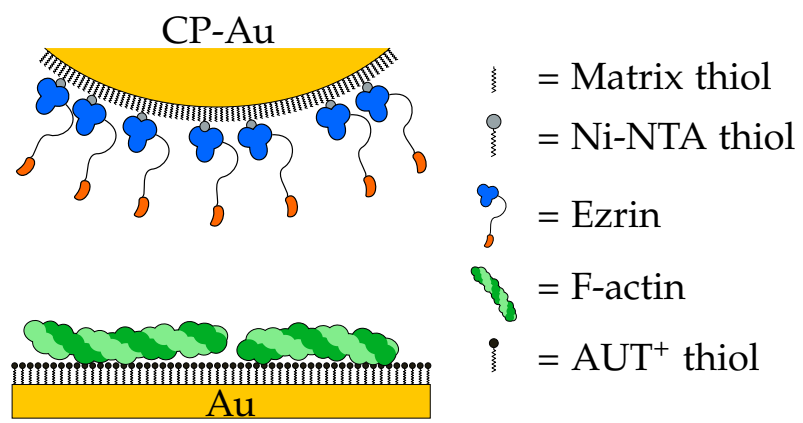

Figure 6.4: Setup for force measurements and functionalization: Schematic drawing of a gold coated Biolever (BL-Au) $(A)$ and a gold colloidal probe (CP-Au) $(\mathbf{B})$ with ezrin functionalization for measuring the interaction with F-actin coated sample.

Depending on the type of experiment, i.e., SMFM/MFS or CPM, different cantilevers were employed. Whereas Biolevers used in MFS or SMFM experiments are characterized by a tip radius of $\sim 30 \mathrm{~nm}$, colloidal probes exhibited radii from $1.7 \mu \mathrm{m}$ to $3.7 \mu \mathrm{m}$. A SEM (scanning electron microscopy) image of a colloidal probe is shown in figure 6.5 A. The colloid radius was determined for each cantilever after the experiment by means of SEM (figure 6.5 B).
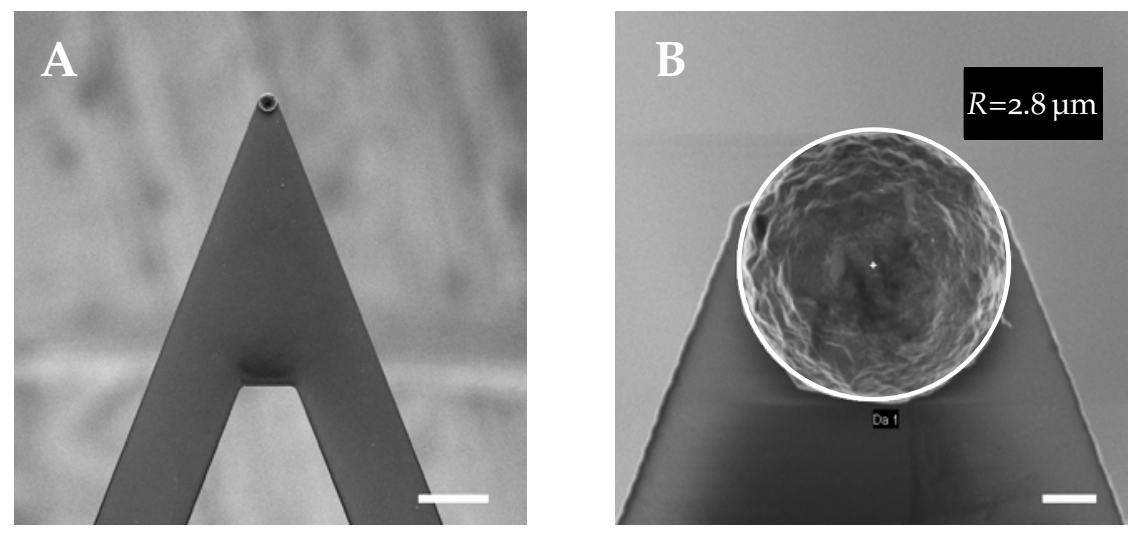

Figure 6.5: SEM images of cantilevers used in colloidal probe microscopy: A Colloidal probe, scale bar: $20 \mu \mathrm{m}$. B Determination of colloidal sphere radius $R$, scale bar: $1 \mu \mathrm{m}$.

\section{Validation of functionalization strategy}

Surface plasmon resonance (SPR) measurements were performed to control the colloidal probe functionalization strategy with regard to its specificity by examining binding and subsequent imidazole induced elution of ezrin bound via the $\mathrm{His}_{6}$ tag to NTA-Ni moieties (section 3.3.3.2). On the one hand, ezrin wildtype, ezrin $\mathrm{T}_{567 \mathrm{~A}}$, and ezrin $\mathrm{T}_{567} \mathrm{D}$ were directly immobilized to the NTA-Ni-containing SAM 
(NTA-thiol/matrix thiol 1:5). On the other hand, the different proteins were preincubated with submicellar $\mathrm{PIP}_{2}$ to mimic the $\mathrm{PIP}_{2}$-induced activation and subsequently bound to the NTA-Ni moieties.

The sensorgram (figure 6.6, a-g) shows the adsorption of $1 \mu \mathrm{M}$ ezrin wildtype pre-incubated with $1 \mu \mathrm{M} \mathrm{PIP}_{2}$ to a mixed SAM (1:5), composed of NTA thiol and matrix thiol, and its imidazole induced desorption. Differences in the refractive indices of buffer solutions caused steplike reflectivity changes upon buffer exchange. The SAM was prepared for specific protein adsorption by charging the NTA head groups with $\mathrm{Ni}^{2+}$ (100 $\mathrm{mm} \mathrm{NiCl}, \mathrm{pH} 8$ adjusted with Tris/ $\left.\mathrm{HCl}\right)$ and rinsing with ultrapure water and $\mathrm{E}_{1}$ buffer to remove excess $\mathrm{Ni}^{2+}(\mathrm{a}-\mathrm{c})$. An increase in reflectivity of 0.13 a.u. was detected upon binding of ezrin wildtype $+\mathrm{PIP}_{2}$ to the NTA-Ni doped surface (d). Rinsing with Eı buffer produced only a minor decrease in reflectivity (e), thus indicating protein binding.

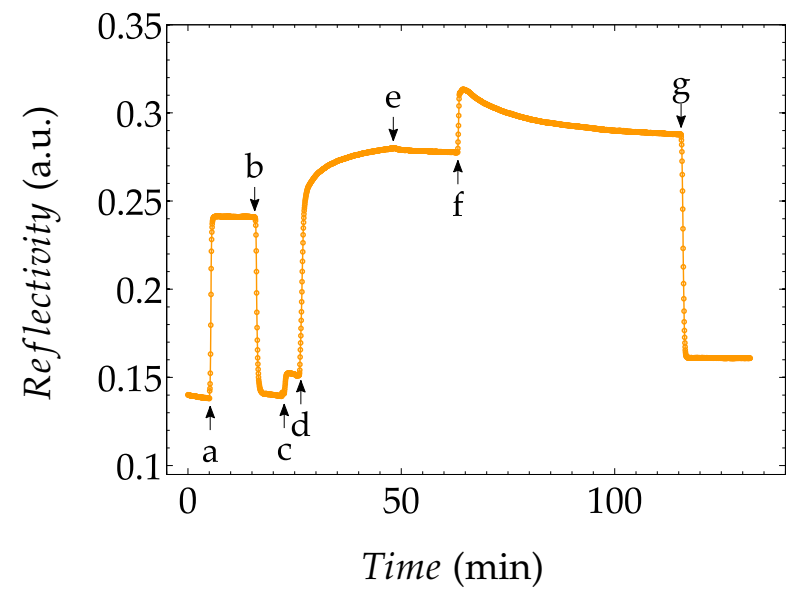

Figure 6.6: Validation of colloidal probe functionalization strategy. SPR measurement showing ezrin wildtype $+\mathrm{PIP}_{2}$ adsorption to NTA-Ni-containing SAM (NTA-thiol/ matrix thiol 1:5) and imidazole induced elution: charging NTA-terminated thiol with $\mathrm{Ni}^{2+}(\mathbf{a})$, rinsing with ultrapure water $(\mathbf{b})$ and E1 buffer $(\mathbf{c})$, binding of $1 \mu \mathrm{M}$ ezrin wildtype $+1 \mu \mathrm{M} \mathrm{PIP}_{2}(\mathrm{~d})$, rinsing with E1 buffer (e), $500 \mathrm{~mm}$ imidazole (f), and E1 buffer (g).

In contrast, the wash step using $500 \mathrm{~mm}$ imidazole (f) and subsequent rinsing with E1 buffer ( $\mathrm{g}$ ) to evaluate the degree of protein desorption lead to a decrease in reflectivity of 0.12 a.u. In total, a minor percentage of $8 \%$ remained bound, caused by nonspecific ezrin wildtype adsorption to the NTA-Ni doped surface, whereas the remaining $92 \%$ could be attributed to specific protein binding to NTA-Ni head groups. Adsorption of ezrin wildtype, ezrin $\mathrm{T}_{56} 6 \mathrm{~A}$, and ezrin $\mathrm{T}_{56}{ }_{7} \mathrm{D}$ in the absence of $\mathrm{PIP}_{2}$ and their imidazole induced desorption gave similar results, although more nonspecifically bound protein was detected $((16 \pm 8) \%)$. However, this higher fraction may be attributed to the fact that the elution period had been halved in these measurements. Furthermore, it has been shown that mono-dispersed $\mathrm{PIP}_{2}(1 \mu \mathrm{M})$ does not adsorb to NTA-Ni doped surfaces on its own. 
We chose to use submicellar $\mathrm{PIP}_{2}$ concentration in order to obtain mono-dispersed $\mathrm{PIP}_{2}$, which is assumed to induce a conformational change of ezrin in solution. ${ }^{176]}$ Values for the critical micellar concentration of $\mathrm{PIP}_{2}$ were found to be in the range of $40 \mu \mathrm{M}$ to $60 \mu \mathrm{M} .^{[176,211]}$ Moreover, we tested whether a similar amount of the ezrin/PIP ${ }_{2}$-complex and ezrin, respectively, was able to bind to the NTA-Ni-containing SAM in the same SPR experiment, regenerating the NTA-Ni-containing SAM by imidazole induced protein elution. First, an ezrin/PIP $\mathrm{P}_{2}$ ratio of 1:10 was used with a $\mathrm{PIP}_{2}$ concentration of $7 \mu \mathrm{M}$. However, we found that the excess of $\mathrm{PIP}_{2}$ appeared to block the $\mathrm{His}_{6}$-tag as a significantly lower amount of ezrin/PIP ${ }_{2}$ (figure 6.7 A, a-b) as compared to ezrin T567D (figure 6.7 A, $\mathrm{a}^{\prime}-\mathrm{b}^{\prime}$ ) was bound to the NTANi-containing SAM .

A

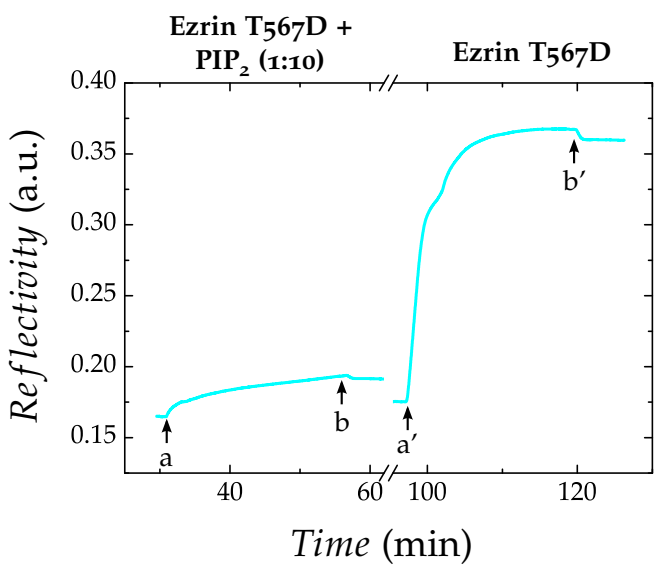

B

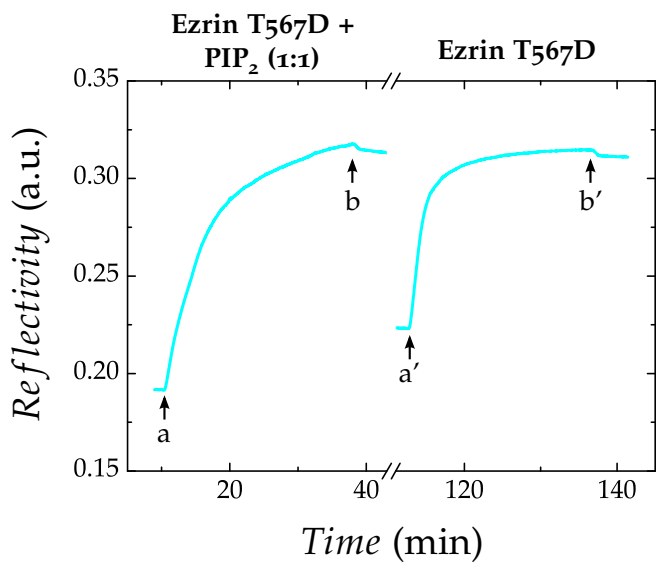

Figure 6.7: Determination of ezrin/PIP ${ }_{2}$ ratio. Extracts from SPR sensorgrams showing the binding of ezrin pre-incubated with PIP $_{2}$ to NTA-Ni-containing SAM, subsequent imidazole induced elution of bound protein (corresponds to axis break), and binding of ezrin $\mathrm{T}_{56} 6 \mathrm{D}$ in absence of $\mathrm{PIP}_{2}$ in one experiment. A Adsorption of $0.7 \mu \mathrm{M}$ ezrin $\mathrm{T}_{5} 67 \mathrm{D}$ preincubated with $\mathrm{PIP}_{2}(1: 10)$ and rinsing with E1 buffer $(\mathrm{a}, \mathrm{b})$. Binding of ezrin $\mathrm{T}_{5} 67 \mathrm{D}$ to regenerated NTA-Ni-containing SAM and subsequent rinsing with E1 buffer $\left(a^{\prime}, b^{\prime}\right)$. B Adsorption of $0.7 \mu \mathrm{M}$ ezrin $\mathrm{T}_{5} 67 \mathrm{D}$ pre-incubated with $\mathrm{PIP}_{2}$ (1:1) and rinsing with E1 buffer $(a, b)$. Binding of ezrin $\mathrm{T}_{5} 67 \mathrm{D}$ to regenerated NTA-Ni-containing SAM and subsequent rinsing with E1 buffer $\left(a^{\prime}, b^{\prime}\right)$.

Employing a 1:1 ratio of ezrin $/ \mathrm{PIP}_{2}$ and thus reducing the concentration of $\mathrm{PIP}_{2}$ to $0.7 \mu \mathrm{M}$ was successful as similar reflectivity values were obtained when either ezrin $\mathrm{T}_{567} \mathrm{D} / \mathrm{PIP}_{2}$ (figure $6.7 \mathrm{~B}, \mathrm{a}-\mathrm{b}$ ) or ezrin $\mathrm{T}_{567} \mathrm{D}$ (figure $6.7 \mathrm{~B}, \mathrm{a}^{\prime}-\mathrm{b}^{\prime}$ ) was bound to the same NTA-Ni-containing SAM.

Confocal laser scanning microscopy (CLSM) was applied to validate the F-actin surface functionalization. The adsorption of AlexaFluor488-phalloidin labeled Factin to an $\mathrm{AUT}^{+}$functionalized wafer is shown in figure 6.8. Even after vigorous rinsing, F-actin remained attached to the surface owing to strong electrostatic interactions. Phalloidin is not only used for mere visualization purposes, but also for stabilization of F-actin. It is known to bind preferably to F-actin rather than to monomeric actin, thus shifting the equilibrium between filaments and monomers 
towards the filamentous form. ${ }^{[213]}$ The length distribution of F-actin was rather inhomogeneous ranging from several $100 \mathrm{~nm}$ to few micrometer. The average F-actin surface coverage amounted to $(67 \pm 7) \%(n=7)$ as determined by pixel analysis in ImageJ.

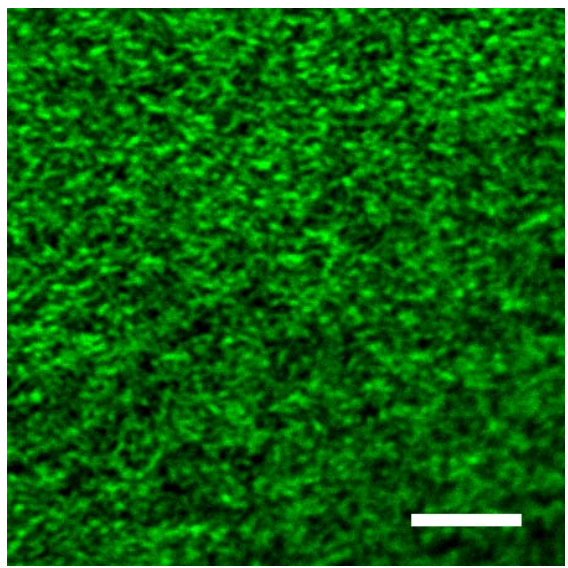

Figure 6.8: Validation of F-actin surface functionalization strategy. Confocal fluorescent image of AlexaFluor488-phalloidin labeled F-actin immobilized on $\mathrm{AUT}^{+}$functionalized gold coated wafer. Scale bar: $5 \mu \mathrm{m}$.

Typically, we recorded in one experiment $\sim 120$ deflection displacement curves. The colloidal probes were controlled after the experiment with regard to unspecific adsorption of F-actin. Rarely, we observed adsorbed F-actin due to poor functionalization, which lead to unusual high adhesion forces and was verified by fluorescence microscopy after the experiment (figure 6.9). Data originating from such measurements was discarded.
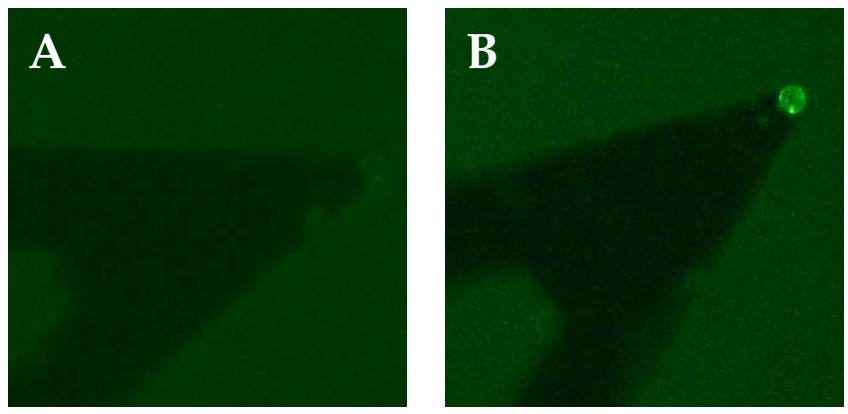

Figure 6.9: Cantilever control regarding adhered F-actin. Fluorescent image of colloidal probe before (A) and after (B) the experiment. AlexaFluor488-phalloidin labeled F-actin adsorbed to colloid due to poor functionalization. Data resulting from such experiments was discarded.

\subsubsection{Measuring the forces between F-actin and ezrin as a function of its activation}

The following experiments were motivated by the question whether and how the activation of ezrin influences the interaction between ezrin and F-actin with regard to its adhesion forces $F_{\mathrm{ad}}$ and surface adhesion energies $W_{\mathrm{ad}}$. An intramolecular 
head-to-tail association conformationally regulates the F-actin binding capability of ezrin. Dissociation and consequently release of the F-actin binding site is thought to require $\mathrm{PIP}_{2}$ binding and phosphorylation of the threonine at position $567 .^{[1]}$ To investigate the individual contribution of the respective activation factor, we made use of ezrin wildtype, pseudophosphorylated ezrin $\mathrm{T}_{56} 6_{7} \mathrm{D}$, nonphosphorylatable ezrin $\mathrm{T}_{567} \mathrm{~A}$, respectively, with or without submicellar $\mathrm{PIP}_{2}$ concentrations. We established a CPM assay consisting of an ezrin coated cantilever probing an F-actin functionalized surface to monitor specifically the impact of ezrin activation on the interaction. Colloidal probes were chosen over usual cantilevers as the colloid sample interaction scenario resembles rather the situation within the cell, where many ezrin molecules are presented to F-actin.

Our strategy to use ezrin wildtype, ezrin $\mathrm{T}_{567} \mathrm{~A}$, and ezrin $\mathrm{T}_{56} 6 \mathrm{D}$ with and without $\mathrm{PIP}_{2}$ to map the influence of no, partial, and full activation generated the six different interaction scenarios summarized in table 6.1.

Table 6.1: Six different ezrin-F-actin interaction scenarios were used to monitor the impact of $\mathrm{PIP}_{2}$ and pseudophosphorylation. Their putative activation state is indicated by + .

\begin{tabular}{l|ccc}
\hline & No activation & Partial activation & Full activation \\
\hline Ezrin $\mathrm{T}_{567 \mathrm{~A}}$ & + & - & - \\
\hline EzrinT567A $+\mathrm{PIP}_{2}$ & - & + & $-/+$ \\
\hline Ezrin wildtype & + & - & - \\
\hline Ezrin wildtype $+\mathrm{PIP}_{2}$ & - & + & $-/+$ \\
\hline Ezrin T567D & - & + & - \\
\hline Ezrin $\mathrm{T}_{567} \mathrm{D}+\mathrm{PIP}_{2}$ & - & - & + \\
\hline
\end{tabular}

Ezrin $\mathrm{T}_{567} \mathrm{~A}$ and ezrin wildtype are considered to be inactive in absence of $\mathrm{PIP}_{2}$. A partial activation is assumed to be induced by the pseudophosphorylation in case of ezrin $\mathrm{T}_{567} \mathrm{D}$. The influence of $\mathrm{PIP}_{2}$ has been controversially discussed in literature. Its contribution to ERM activation was confirmed. However, some studies indicate that $\mathrm{PIP}_{2}$ alone might be able to induce full activation of ERM proteins. ${ }^{[11,13]}$ The combination of the pseudophosphorylated ezrin $\mathrm{T}_{5} 67 \mathrm{D}$ and $\mathrm{PIP}_{2}$ represents in our work the fully activated ezrin. Considering the limiting cases of the six different interaction scenarios, we expected that no interaction with F-actin is observed for ezrin T567A and ezrin wildtype, respectively (figure 6.10 A). Accordingly, no deviations between the colloidal probe deflection of approach (blue) and retraction curve (red), respectively, should occur. On the contrary, ezrin $\mathrm{T}_{567} \mathrm{D}$ in presence of $\mathrm{PIP}_{2}$ was expected to be fully activated, thus being able to bind efficiently to F-actin (figure 6.10 B). For this reason, adhesion events due to the interaction between Factin and ezrin $\mathrm{T}_{56} 6 \mathrm{D}$ in presence of $\mathrm{PIP}_{2}$ were expected to generate a characteristic 
deflection pattern upon retraction.

A

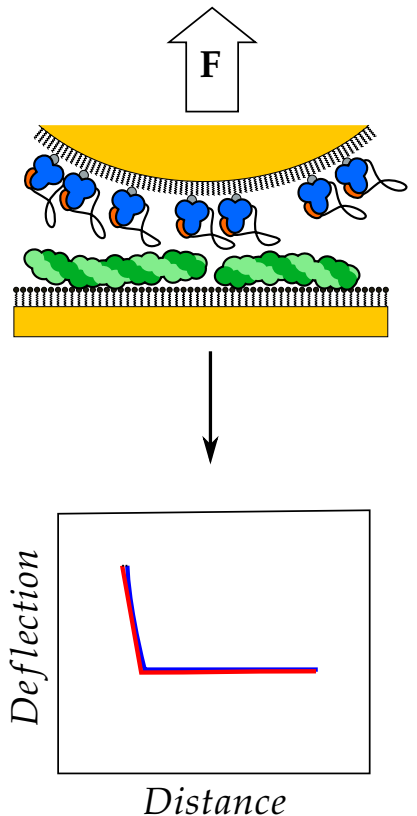

B
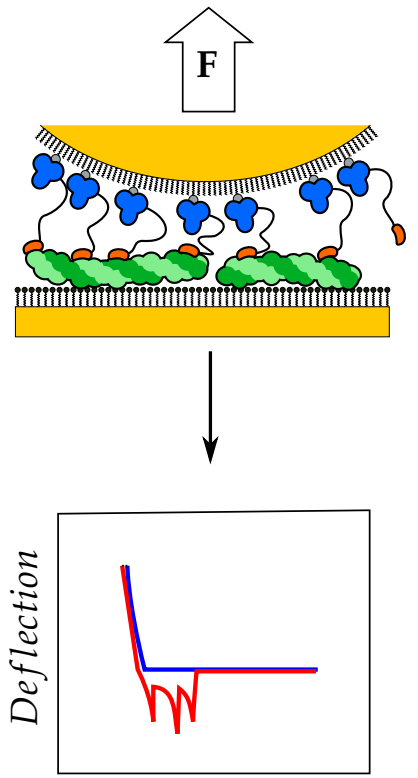

Distance

Figure 6.10: Limiting cases of interaction between ezrin coated colloidal probe and F-actin covered surface according to experimental setup shown in figure 6.4 B. A Inactive ezrin is not able to bind F-actin. The deflection course is the same for both approach (blue line) and retraction (red line) curve of the colloidal probe. B Active ezrin interacts with F-actin. The force $F$ acting on the formed bonds caused by the retracting colloidal probe induces bond rupture, leading to abrupt changes in deflection.

All deflection displacement curves originating from the six different interaction scenarios were converted into force distance curves (section 3.3.1.1) and processed as described in the following section 6.2.2. Representative force distance curves describing the limiting cases are shown in figure 6.11. The interaction between ezrin T567 A and F-actin is characterized by several adhesion events, although no interaction was expected. However, the force distance curve showing the interaction between $\mathrm{F}$-actin and ezrin $\mathrm{T}_{567} \mathrm{D}$ in presence of $\mathrm{PIP}_{2}$ exhibits significantly more adhesion events and consequently a larger surface adhesion energy.
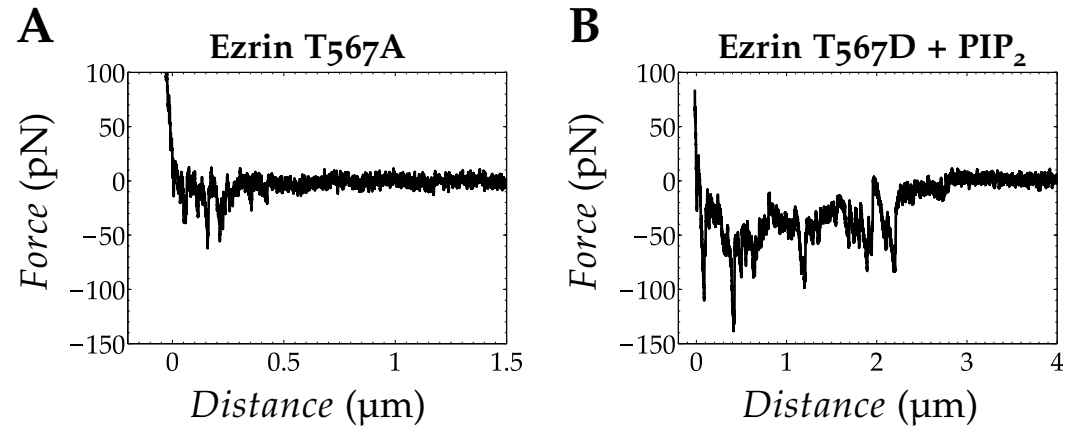

Figure 6.11: Representative force distance curve (retrace) showing interaction between ezrin coated colloidal probe and F-actin covered surface. A Ezrin T567A. B Ezrin T567D + PIP ${ }_{2}$. 
In general, the force distance curves were characterized by a high degree of heterogeneity. One reason for that might be intrinsic to the experimental setup. F-actin on the sample surface was not homogeneously distributed but rather forming a mesh-like structure (figure 6.8). Consequently, the number of formed bonds varied with each contact, leading to rather heterogeneous force distance curves. Therefore, we refrained from evaluating individual force distance curves and chose to use statistical tools to analyze our data with regard to possible differences in adhesion force and surface adhesion energy depending on the respective interaction scenario as described in the following.

\section{Data analysis}

Raw data was processed via the MFP-3D software (Asylum Research, Santa Barbara, USA) based in IGOR Pro (version 6.22a, WaveMetrics, Portland, USA). Thereby, deflection displacement curves were converted to force distance curves according to paragraph 3.3.1.1. The processed data was imported for further analysis into MATLAB using the FDC analysis routine. The first analysis step comprised baseline correction and setting the contact point manually. Local minima (figure 6.12, red stars) were determined via a peak finding routine, yielding the adhesion forces $F_{\mathrm{ad}}$.

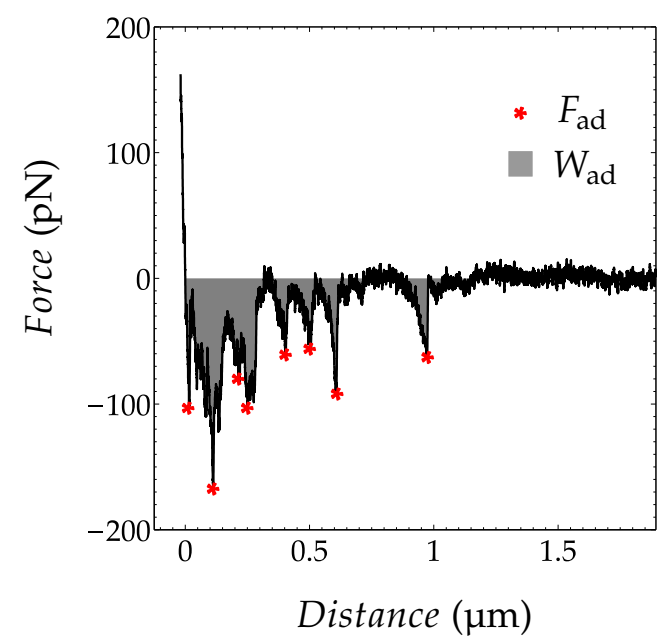

Figure 6.12: Typical force distance curve in colloidal probe microscopy. For data analysis, the adhesion peaks (red stars) and the area under the curve (gray area) were evaluated, yielding adhesion forces $\left(F_{\mathrm{ad}}\right)$ and surface adhesion energy $\left(W_{\mathrm{ad}}\right)$, respectively.

Integration of the area under the curve produced the respective surface adhesion energy $W_{\text {ad }}$ (figure 6.12, gray area). Depending on the data quality, i.e., signal/noise ratio or interference issues, the analysis was either automated or carried out by hand. 
In the second step, the browse tool was used to pool the results for each experiment resulting in two data sets, $F_{\mathrm{ad}}$ and $W_{\mathrm{ad}}$. Data sets originating from different experiments were combined, provided they were performed under the same conditions. Next, each data set was evaluated via a self-written MATLAB routine, estimating the respective probability density function (pdf) and the cumulative distribution function (cdf) (figure 6.13). For visualizing the obtained estimated densities, either histogram analysis or kernel density estimation was performed. In case of histogram density estimation, the optimal bin number was computed according to Freedman-Diaconis. ${ }^{[214]}$ The function rhist yielded the relative frequency $f_{w}(x)$, i.e., the number of times a particular value for a variable occurs divided by the total number $n$ of observations:

$$
f_{w}(x)=\frac{1}{n} \sum_{i=1}^{n} B\left(x-\tilde{X}_{i}\right) \quad \text { with } \quad B= \begin{cases}1 & \text { if } x \in(-w / 2, w / 2) \\ 0 & \text { otherwise }\end{cases}
$$

$\tilde{X}_{i}$ specifies the center of the bin where observation $X_{i}$ lies and B denotes the indicator function, which equals 1 if its argument holds and 0 otherwise. The bin width $w$ was calculated from the distance between two adjacent bin centers. To obtain a density histogram, the relative frequency was divided by the bin width $w$. Accordingly, the pdf estimator $\hat{p}_{w}$ is given by:

$$
\widehat{p}_{w}=\frac{1}{w} f_{w}(x)
$$

However, drawbacks have to be taken into account as histograms are used for probability density estimation including the dependence on bin width, bin origin and the intrinsic discontinuous density. The two latter ones are circumvented by using the kernel density estimation approach. Its principle relies on the weighting kernel function $K$ : the closer the observation $X_{i}$ is to $x$, the greater is the allocated weight. Therefore, kernel density estimation was employed as complementary pdf estimation approach by the MATLAB routine (figure 6.13). The pdf estimator $\widehat{p}_{h}(x)$ for kernel density estimation is given by:

$$
\widehat{p}_{h}(x)=\frac{1}{n h} \sum_{i=1}^{n} K\left(\frac{x-X_{i}}{h}\right)
$$

where $h$ denotes the kernel bandwidth, also referred to as smoothing parameter. Its choice is critical with regard to over- or undersmoothing of the density estimate. 
A widely used kernel function is the so-called Epanechnikov kernel based on a second-degree polynomial: ${ }^{[215]}$

$$
K(u)= \begin{cases}\frac{3}{4}\left(1-u^{2}\right) & \text { if }|u| \leq 1 \\ 0 & \text { otherwise }\end{cases}
$$

Test kernel density estimations with varying bandwidth were performed to determine a suitable width leading to smooth but not oversmoothed density estimations. The kernel probability density estimation was carried out by using the ksdensity function with Epanechnikov kernel. As result of the pdf estimation, the histogram was overlaid with the pdf obtained from kernel density estimation.

The empirical cdf describes the proportion of observed values that are less than or equal to each value in turn. In case of the cdf, the estimator is given by:

$$
\widehat{F}(x)=\frac{1}{n} \sum_{i=1}^{n} I\left(X_{i} \leq x\right) \quad \text { with } \quad I\left(X_{i} \leq x\right)= \begin{cases}1 & \text { if } X_{i} \leq x \\ 0 & \text { otherwise }\end{cases}
$$

where $I$ denotes the indicator function, which equals 1 if its argument holds and 0 otherwise. The empirical cdf assigns a probability mass of $1 / n$ at each observation. 


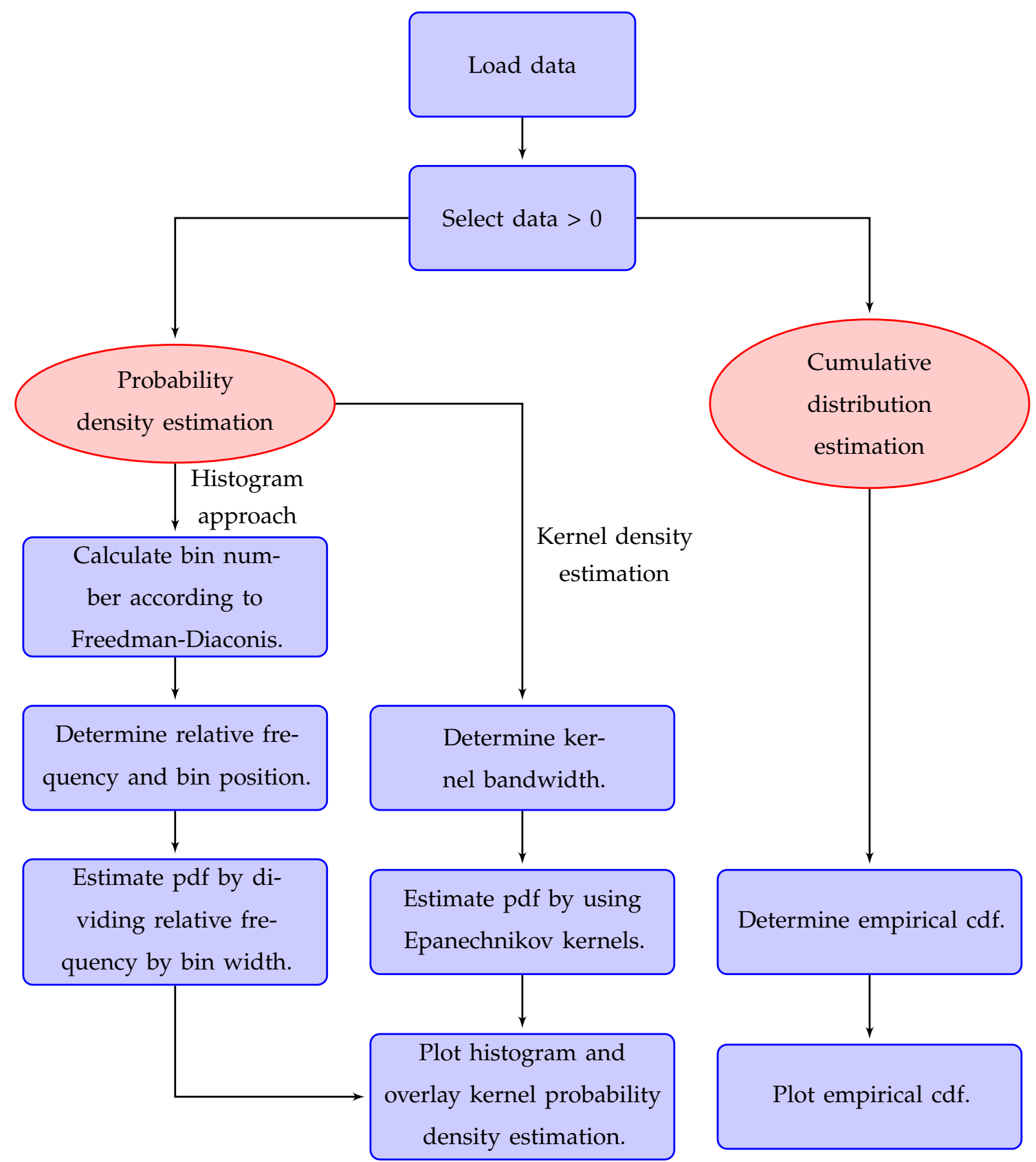

Figure 6.13: Flow chart of MATLAB routine for probability density estimation of force measurement data (pdf: probability density function, cdf: cumulative distribution function). 
6.2.3 Colloidal probe microscopy revealing differences and similarities in adhesion between F-actin and ezrin as a function of its activation

In this section, we present the adhesion forces $F_{\text {ad }}$ and surface adhesion energies $W_{\text {ad }}$ of the interaction between F-actin and ezrin determined by means of colloidal probe microscopy (CPM). In particular, the influence of stepwise or increasing ezrin activation on the ezrin F-actin interaction will be considered by evaluating the influence of $\mathrm{PIP}_{2}$ binding and phosphorylation separately as described in table 6.1.

The two-dimensional Kolmogorov-Smirnov (KS) test was employed to compare the distributions with regard to significant differences. One advantage of this test is that no assumptions are made about the underlying type of distribution. Based on the Bonferroni correction, a reduced significance level of $\alpha^{\prime}=3.1 \times 10^{-3}$ was set to take multiple testing into account, thus guaranteeing an overall significance level of $\alpha=0.05$. The two-dimensional KS test is based on the empirical distribution function and tests whether two samples come from the same continuous distribution. We found that all distributions of $F_{\text {ad }}$ (figure 6.14) differ significantly from each other. The same result was obtained when comparing the distributions of $W_{\mathrm{ad} \text {, norm }}$ (figure 6.18).

\section{Adhesion forces between F-actin and ezrin}

The most probable adhesion force $F_{\text {ad }}^{*}$ and the median $\tilde{F}_{\text {ad }}$ were determined from the respective distributions. As we employed CPM where many ezrin molecules are attached to the colloid, the probability of a binding event is high. Accordingly, $F_{\text {ad }}^{*}$ represents the most probable adhesion force of the interaction between ezrin and F-actin. The median value halves the distribution as such that there are the same number of adhesion forces below the median as there are above the median. In case of a perfectly symmetrical distribution the most probable value and the median coincide. However, the distributions of measured adhesion forces are skewed to the right (figure 6.14). Accordingly, the most probable value is less than the median.

In figure 6.14 all adhesion force distributions are depicted, describing the different interaction scenarios between F-actin and ezrin as a function of its putative activation state (table 6.1). 


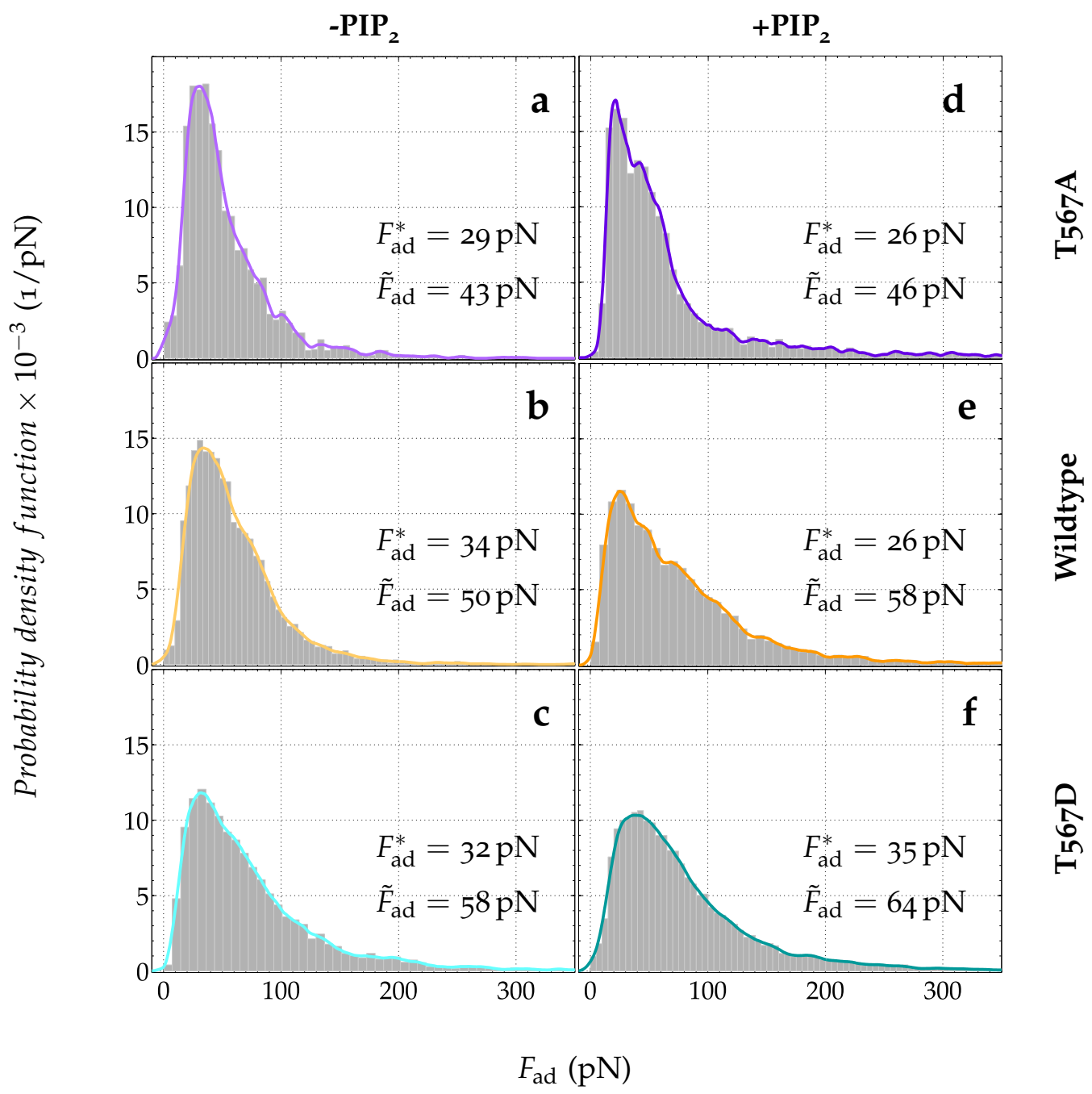

Figure 6.14: Probability density function of adhesion forces $F_{\text {ad }}$ between F-actin and ezrin as a function of its activation: ezrin T567A $(n=2825)$, ezrin wildtype $(n=6128)$, and ezrin $\mathrm{T}_{567} \mathrm{D}(n=4747)$ in absence of $\mathrm{PIP}_{2}(\mathrm{a}-\mathrm{c})$ and ezrin $\mathrm{T}_{567} \mathrm{~A}(n=3849)$, ezrin wildtype $(n=6675)$, and ezrin $\mathrm{T}_{567} \mathrm{D}(n=17216)$ in presence of $\mathrm{PIP}_{2}(\mathbf{d}-\mathbf{f})$. The most probable adhesion force $F_{\text {ad }}^{*}$ as well as the median $\tilde{F}_{\text {ad }}$ were determined for each distribution.

In case of the interaction between ezrin $\mathrm{T}_{5} 67 \mathrm{~A}$ and F-actin, the most probable adhesion force $F_{\text {ad }}^{*}$ amounts to approximately $29 \mathrm{pN}$, while the median adhesion force was determined with $\tilde{F}_{\text {ad }}=43 \mathrm{pN}$ (figure $6.14 \mathrm{a}$ ). In presence of $\mathrm{PIP}_{2}, F_{\text {ad }}^{*}$ slightly decreases to $26 \mathrm{pN}$ in contrast to the increased $\tilde{F}_{\text {ad }}$ of $46 \mathrm{pN}$ (figure $6.14 \mathrm{~d}$ ). This can be attributed to the fact, that the adhesion force distribution, describing the interaction between F-actin and ezrin $\mathrm{T}_{5} 67 \mathrm{~A}$ in presence of $\mathrm{PIP}_{2}$, exhibits a shoulder at $\sim 42 \mathrm{pN}$. We observed an increase in both $F_{\text {ad }}^{*}$ to $34 \mathrm{pN}$ and $\tilde{F}_{\text {ad }}$ to $50 \mathrm{pN}$ upon ezrin wildtype interacting with F-actin (figure 6.14 b). PIP 2 affects $F_{\text {ad }}^{*}$ as observed for ezrin T567A yielding $26 \mathrm{pN}$. Similarly, $\tilde{F}_{\text {ad }}$ increases in presence of $\mathrm{PIP}_{2}$ to $58 \mathrm{pN}$ (figure $6.14 \mathrm{e}$ ). In case of the interaction between F-actin and the pseudophosphorylated mutant ezrin $\mathrm{T}_{5} 67 \mathrm{D}, F_{\mathrm{ad}}^{*}$ is further increased to $32 \mathrm{pN}$. Likewise, a higher median value $\tilde{F}_{\text {ad }}$ of $75 \mathrm{pN}$ was determined (figure $6.14 \mathrm{c}$ ). Both values are further 
increased upon presence of $\mathrm{PIP}_{2}$ to $F_{\mathrm{ad}}^{*}=35 \mathrm{pN}$ and $\tilde{F}_{\mathrm{ad}}=58 \mathrm{pN}$ (figure 6.14 f).

The adhesion forces of the interaction between N-ERMAD, the $N$-terminal part of ezrin lacking the $C$-terminal F-actin binding site, and F-actin are depicted in figure 6.15. This interaction was used as control to evaluate whether the interactions observed can be attributed to the specific interaction between ezrin and F-actin via the C-terminal F-actin binding site. We found $F_{\text {ad }}^{*}$ to be $22 \mathrm{pN}$, while $\tilde{F}_{\text {ad }}$ amounts to $28 \mathrm{pN}$. It is expected to observe some unspecific interaction as a protein covered probe is brought into contact with the F-actin surface. However, the observed adhesion forces are lower than the ones determined for specific binding. Furthermore, the nature of this unspecific interaction is clearly distinguishable from specific interactions - only very narrow and few peaks were observable - as it is shown in figure 6.15. This effect has been shown to be most pronounced with regard to the corresponding surface adhesion energies (figure 6.18 a). Almost no contribution to the surface adhesion energies resulted from these peaks as will be explained in more detail in the following.
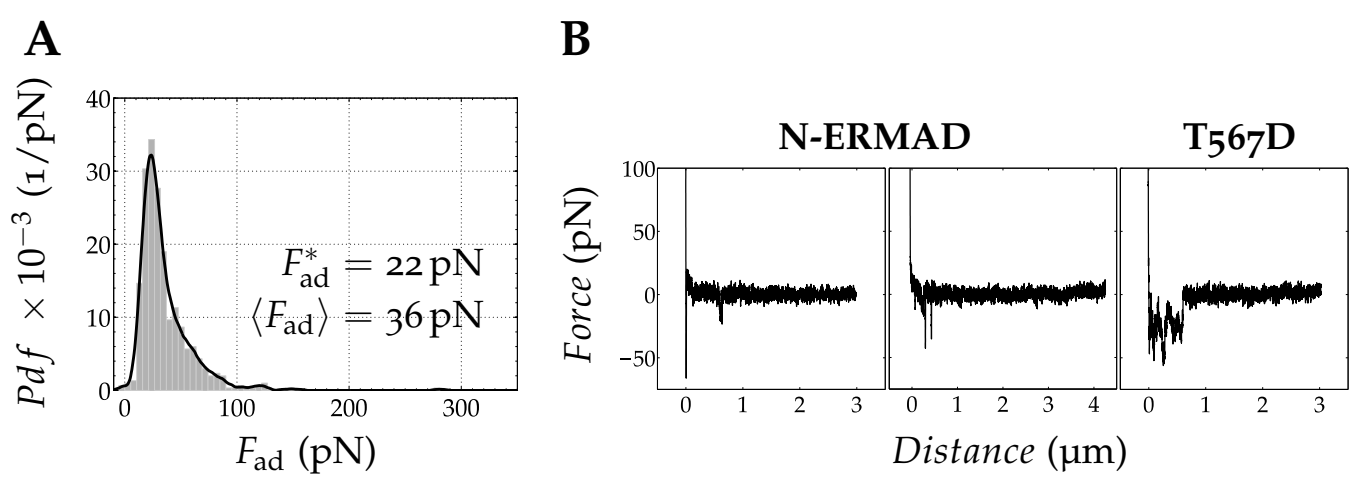

Figure 6.15: A Probability density function of adhesion forces $F_{\text {ad }}$ between F-actin and NERMAD $(n=564)$. The most probable as well as the median adhesion force $F_{\text {ad }}^{*}$ and $\tilde{F}_{\text {ad }}$ were determined. B Representative force distance curves probing the interaction between F-actin and either N-ERMAD or ezrin T567D.

The empirical cdf was used as a different visualization to compare all distributions within one diagram. The impact of ezrin activation on the adhesion forces $F_{\text {ad }}$ to F-actin is not as pronounced as one might expect in the first place. The most probable adhesion forces $F_{\text {ad }}^{*}$ range from $26 \mathrm{pN}$ to $35 \mathrm{pN}$, while the median adhesion forces $\tilde{F}_{\text {ad }}$ cover a range from $43 \mathrm{pN}$ to $64 \mathrm{pN}$. This behavior is reflected in the empirical cdf (figure 6.16). 


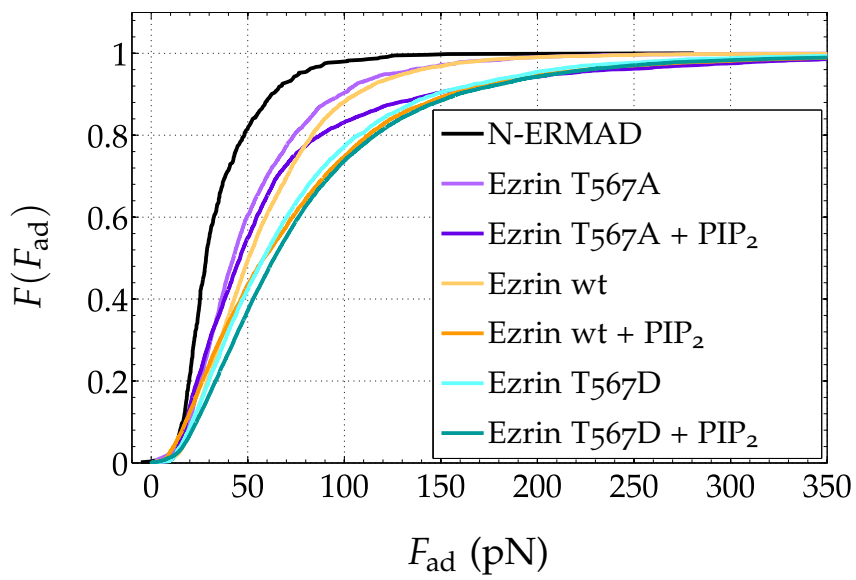

Figure 6.16: Empirical cumulative density function of adhesion forces.

The empirical cdf in case of N-ERMAD (black) differs from the others as it is shifted to lower $F_{\text {ad }}$ owing to its lack of F-actin binding site. Ezrin T567A and ezrin wildtype in absence of $\mathrm{PIP}_{2}$ (light purple and light orange, respectively) are theoretically able to bind to F-actin, although their $C$-terminal F-actin binding site is expected to be blocked owing to intramolecular self-association (figure 6.2). As expected, their empirical cdfs are the closest to N-ERMAD. The pseudophosphorylated mutant ezrin $\mathrm{T}_{567} \mathrm{D}$ (light turquoise) is assumed to be partially activated as the aspartate mimics the negatively charged phosphate group. In comparison to ezrin $\mathrm{T}_{5} 67 \mathrm{~A}$ and ezrin wildtype, the empirical cdf for ezrin $\mathrm{T}_{5} 67 \mathrm{D}$ is further shifted to higher $F_{\text {ad }}$.

$\mathrm{PIP}_{2}$ is the natural occurring receptor lipid of ezrin and believed to be involved in its recruitment to the membrane as well as to be part of its activation mechanism. In presence of $\mathrm{PIP}_{2}$, the empirical cdfs of ezrin $\mathrm{T}_{5} 67 \mathrm{~A}$ and ezrin wildtype (dark purple and dark orange, respectively) differ from each other as well as from the ones in absence of $\mathrm{PIP}_{2}$. The latter one agrees with the concept of the activating influence of $\mathrm{PIP}_{2}$. However, it was not expected to observe unalike impact of $\mathrm{PIP}_{2}$ on ezrin T567A and ezrin wildtype. The empirical cdf of ezrin T567A with and without $\mathrm{PIP}_{2}$ are closely matching at low $F_{\mathrm{ad}}$, which is similar to ezrin wildtype. At approximately $40 \mathrm{pN}$, the curves start to diverge. The degree of divergence, however, is more prominent for ezrin wildtype in presence of $\mathrm{PIP}_{2}$ as compared to ezrin $\mathrm{T}_{5} 67 \mathrm{~A}$ under same conditions. Interestingly, the presence of $\mathrm{PIP}_{2}$ has minor impact on $F_{\mathrm{ad}}$ of ezrin $\mathrm{T}_{5} 6_{7} \mathrm{D}$ as its empirical cdf (dark turquoise) is only shifted slightly towards higher $F_{\mathrm{ad}}$. If we consider the influence of the pseudophosphorylation in presence of $\mathrm{PIP}_{2}$ by comparing ezrin wildtype and ezrin $\mathrm{T}_{5} 67 \mathrm{D}$, the empirical cdf of ezrin $\mathrm{T}_{5} 67 \mathrm{D}$ is shifted to higher $F_{\mathrm{ad}}$ as compared to ezrin wildtype below $\sim 8 \mathrm{o} \mathrm{pN}$. However, this difference diminishes at higher $F_{\text {ad }}$ leading to overlapping empirical cdfs for both ezrin $\mathrm{T}_{5} 67 \mathrm{D}$ and ezrin wildtype in presence of $\mathrm{PIP}_{2}$. 
To summarize and elaborate the characteristics of the distributions, we chose to compare them by using box plots. One major advantage of the box plot is its ability to visualize straightforward critical information about a dataset including the spread of observations and symmetry of the distribution. ${ }^{[216]}$ A typical box plot is characterized by a solid box that spans the interquartile range (IQR). Dashed lines, so-called whiskers extended up to $1.5 \times \mathrm{IQR}$ from the two ends of the box to exclude outliers. The median is located within the IQR and defines the center of a distribution irrespective of its shape. while the location of the median is scarcely affected by outliers and extreme values. The notch in a box plot represents the uncertainty of the central tendency, thus indicating the significance of differences between determined median values. If two notches do not overlap, the respective medians are different at $5 \%$ significance level. ${ }^{[217]}$

Box plot distributions of the measured $F_{a d}$ are depicted in figure 6.17. Already the histogram analysis has revealed that all distributions are skewed to the right (figure 6.14). This asymmetry is visualized in the box plot as such that the median is rather shifted to lower $F_{\mathrm{ad}}$ values than being centered within the IQR, which would be the case for a symmetric distribution. By comparing the different box plots, a trend to higher $F_{\mathrm{ad}}$ emerges with increasing activation of ezrin, although the changes are rather subtle. The box plots of ezrin $\mathrm{T}_{567} \mathrm{~A}$ with and without $\mathrm{PIP}_{2}$ as well as ezrin wildtype resemble each other.

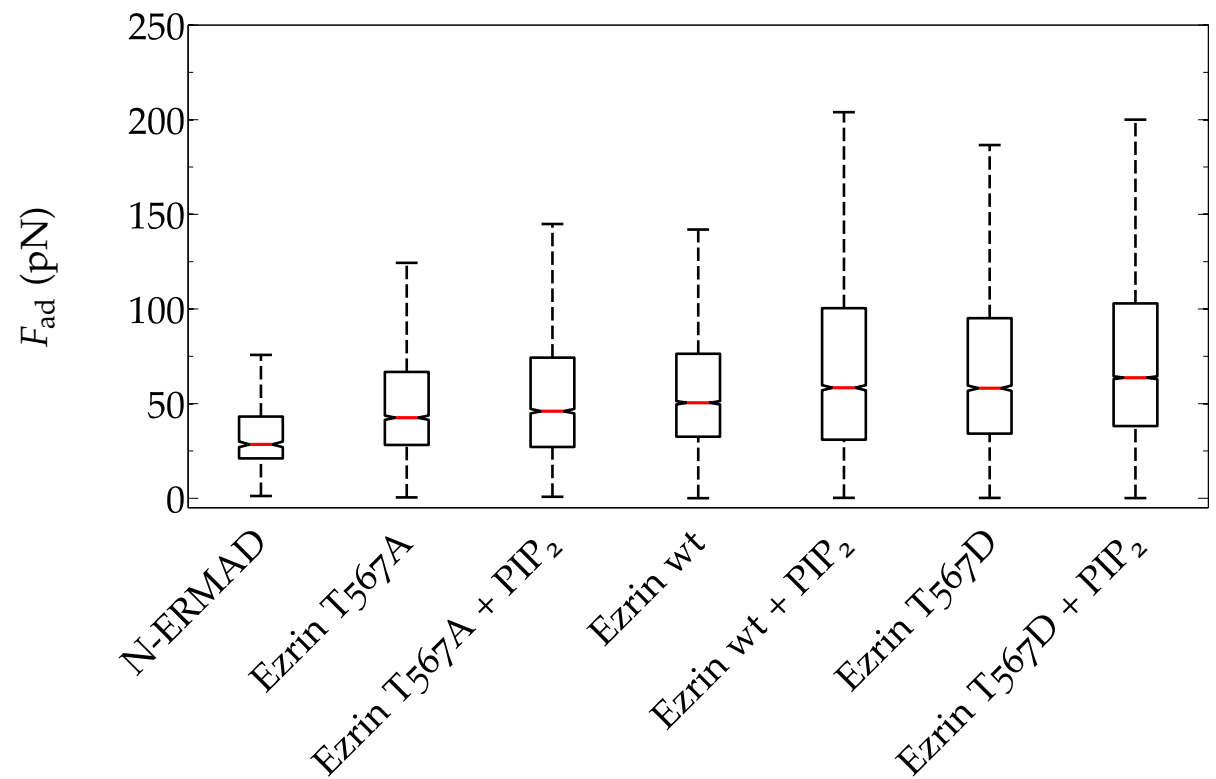

Figure 6.17: Box plot distribution of adhesion forces resulting from the interaction between F-actin and ezrin depending on its activation. N-ERMAD is included as control. The red line represents the median, the solid box is the interquartile range (IQR) and the whiskers (dashed lines) are defined in length by $1.5 \times$ IQR. Data points outside this range are not shown. Notches indicate the location of the median at $95 \%$ confidence.

Spreading of the $F_{\text {ad }}$ distributions is observed upon activation of ezrin as the IQR is extended for ezrin wildtype $+\mathrm{PIP}_{2}$, ezrin $\mathrm{T}_{5} 67 \mathrm{D}$ with and without $\mathrm{PIP}_{2}$ towards 
higher $F_{\mathrm{ad}}$. Although the distribution of $F_{\mathrm{ad}}$ appears to change upon ezrin activation in general, the overall shift is too small to allow any conclusions with regard to an increased $F_{\text {ad }}$ as a result of the activation process.

In case of N-ERMAD, the IQR is shifted to lower $F_{\text {ad }}$ values and the spread of the distribution is not as extended as for all other cases. The observed adhesion events for N-ERMAD might be attributed to a certain F-actin binding affinity that was detected for C-terminally truncated ezrin (amino acids 1-310, 1-330). ${ }^{[218]}$ However, it was found to be considerably lower as compared to the one for full-length ezrin. This is in accordance with our results, showing that the determined $W_{\text {ad, norm }}$ is substantially lower as the one observed for fully activated ezrin, i.e., ezrin $\mathrm{T}_{567} \mathrm{D}$ in presence of $\mathrm{PIP}_{2}$ (figure 6.20), thus indicating the importance of the C-terminal F-actin binding site.

\section{Surface adhesion energies between F-actin and ezrin}

Before estimating the particular surface adhesion energies, each data originating from the same experiment was normalized to account for different colloidal dimensions. The proportionality was derived from the Hertz Model according to which the contact radius $a_{\text {Hertz }}$ of a spherical tip with radius $R$ and a flat surface is given by: $[44]$

$$
a_{\mathrm{Hertz}}=\sqrt[3]{\frac{R F}{E_{\mathrm{tot}}}},
$$

where $F$ is the force applied by the tip on the surface and $E_{\text {tot }}$ is the reduced Young's modulus. Accordingly, the contact area $A_{\mathrm{Hertz}}$ satisfies the following proportionality:

$$
A_{\text {Hertz }} \sim \sqrt[3]{R^{2}}
$$

This proportional relationship is used to normalize the surface adhesion energy data.

The mean normalized surface adhesion energy $\left\langle W_{\mathrm{ad}, \text { norm }}\right\rangle$ as well as the median $\tilde{W}_{\text {ad, norm }}$ were determined from the respective distributions. The most probable normalized surface adhesion energy was not considered as the normalized surface adhesion energy distributions were partly heavily skewed to the right. Accordingly, this value would not represent these distributions properly.

In presence of $\mathrm{PIP}_{2}$, the mean surface adhesion energy $\left\langle W_{\mathrm{ad}, \text { norm }}\right\rangle$ between F-actin and ezrin $\mathrm{T}_{5} 67 \mathrm{~A}$ is increased from $80 \mathrm{fJ} / \mathrm{m}^{2 / 3}$ to $100 \mathrm{fJ} / \mathrm{m}^{2 / 3}$ (figure $6.18 \mathrm{~b}$, e). We 
observed a significantly higher $\left\langle W_{\mathrm{ad}}\right.$, norm $\rangle$ of $220 \mathrm{fJ} / \mathrm{m}^{2 / 3}$ in case of the interaction between ezrin wildtype and F-actin that was even further increased to $\left\langle W_{\mathrm{ad}, \text { norm }}\right\rangle$ $330 \mathrm{fJ} / \mathrm{m}^{2 / 3}$ in presence of $\mathrm{PIP}_{2}$ (figure $6.18 \mathrm{c}, \mathrm{f}$ ).

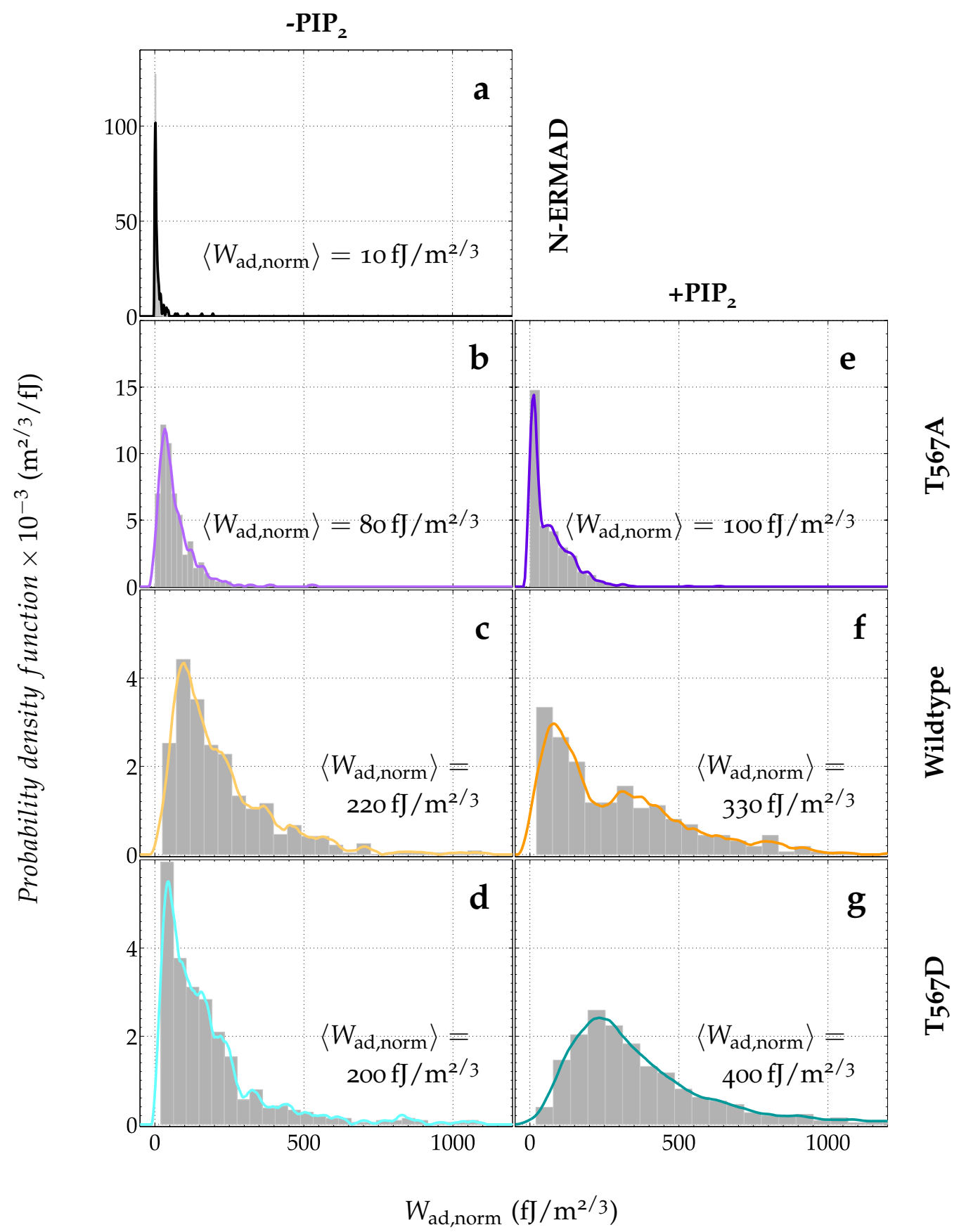

Figure 6.18: Probability density function of normalized surface adhesion energies $W_{\text {ad,norm }}$ between F-actin and ezrin as a function of its activation: control N-ERMAD $(n=156, \mathbf{a})$, ezrin T567A $(n=236)$, ezrin wildtype $(n=519)$, ezrin $T_{567} \mathrm{D}(n=505)$ in absence of $\mathrm{PIP}_{2}$ (b-d) and ezrin T567A $(n=481)$, ezrin wildtype $(n=299)$, and ezrin T567 $\mathrm{D}(n=836)$ with $\mathrm{PIP}_{2}(\mathbf{e}-\mathbf{g})$. The mean normalized surface adhesion energy $\left\langle W_{\mathrm{ad}, \text { norm }}\right\rangle$ was determined for each distribution.

In case of the interaction between the pseudophosphorylated ezrin $\mathrm{T}_{56} 6_{7} \mathrm{D}$ and $\mathrm{F}-$ actin $\left\langle W_{\text {ad, norm }}\right\rangle$ was with $200 \mathrm{fJ} / \mathrm{m}^{2 / 3}$ comparable to ezrin wildtype and F-actin. The influence of $\mathrm{PIP}_{2}$ on the interaction between $\mathrm{F}$-actin and ezrin $\mathrm{T}_{5} 67 \mathrm{D}$ was 
most pronounced, leading to a considerably higher $\left\langle W_{\mathrm{ad}, \text { norm }}\right\rangle$ of $400 \mathrm{fJ} / \mathrm{m}^{2 / 3}$ (figure $6.18 \mathrm{~d}, \mathrm{~g}$ ). Contrary to the results obtained with full length ezrin, the lack of the $C$-terminal F-actin binding site, as for N-ERMAD, leads to significantly lower $\left\langle W_{\mathrm{ad}, \text { norm }}\right\rangle$ of $10 \mathrm{fJ} / \mathrm{m}^{2 / 3}$ (figure $6.18 \mathrm{a}$ ).

In contrast to the adhesion forces between ezrin and F-actin, the impact of ezrin activation on the normalized surface adhesion energies $W_{\text {ad, norm }}$ is significant. The mean normalized surface adhesion energies $\left\langle W_{\mathrm{ad}, \text { norm }}\right\rangle$ span a range from $80 \mathrm{fJ} / \mathrm{m}^{2 / 3}$ to $400 \mathrm{fJ} / \mathrm{m}^{2 / 3}$, which is reflected in the course of the respective empirical cdfs (figure 6.19).

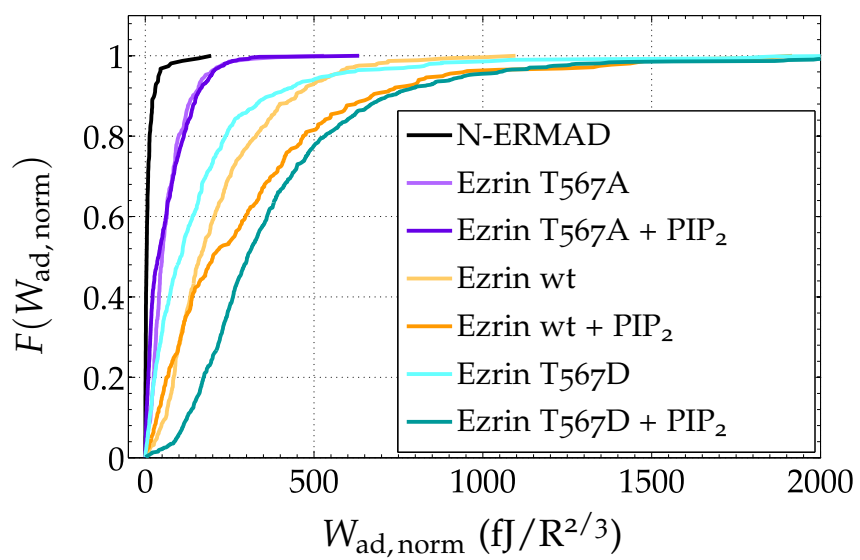

Figure 6.19: Empirical cumulative density function of normalized adhesion energies.

Only low $W_{\text {ad, norm }}$ between N-ERMAD and F-actin were observed, which can be attributed to the lack of the $C$-terminal F-actin binding site found in ezrin. Therefore, the empirical cdf, describing the adhesion energy between N-ERMAD and F-actin, is steeply upward sloping (black). If we consider the effect of pseudophosphorylation in absence of $\mathrm{PIP}_{2}$, the empirical cdfs are shifted towards higher $W_{\mathrm{ad}}$, norm according to the following ordering: ezrin T567A (light purple), ezrin $\mathrm{T}_{5} 67 \mathrm{D}$ (light turquoise), and ezrin wildtype (light orange). This order, however, is contradictory to the expectation that the pseudophosphorylation would enhance the adhesion energy between F-actin and ezrin. In presence of $\mathrm{PIP}_{2}$ the situation has changed. Both empirical cdfs of ezrin wildtype and ezrin $\mathrm{T}_{567} \mathrm{D}$ are shifted with $\mathrm{PIP}_{2}$ to higher $W_{\mathrm{ad}}$, norm (dark orange and dark turquoise, respectively), whereas ezrin T567A remains almost unchanged (dark purple). Striking is that the combination of pseudophosphorylation and PIP $_{2}$ results in the empirical cdf most shifted towards higher $W_{\mathrm{ad} \text {, norm. }}$. This is in agreement with the model that for full activation of ezrin both $\mathrm{PIP}_{2}$ binding and phosphorylation are necessary. Interestingly, the empirical cdf of ezrin wildtype in presence of $\mathrm{PIP}_{2}$ exhibits a step like structure at approximately $250 \mathrm{fJ} / \mathrm{m}^{2 / 3}$. This behavior is owing to the bimodal distribution of $W_{\text {ad, norm }}$ as can be seen in figure $6.18 \mathrm{f}$. At $W_{\text {ad, norm }}$ below $150 \mathrm{fJ} / \mathrm{m}^{2 / 3}$ the empirical 
cdfs of ezrin wildtype with and without $\mathrm{PIP}_{2}$ are similar, while at higher $W_{\text {ad, norm }}$ the empirical cdf of ezrin wildtype in presence of $\mathrm{PIP}_{2}$ approaches the one of fully activated ezrin $\mathrm{T}_{56} 6 \mathrm{D}$ with $\mathrm{PIP}_{2}$.

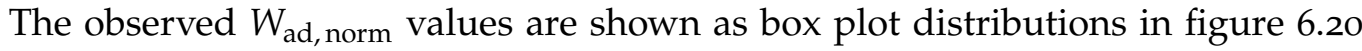
to summarize the obtained distributions and evaluate their spread. As described for the distributions of $F_{\mathrm{ad}}$, all distributions of $W_{\mathrm{ad}}$, norm are skewed to the right (figure 6.20). The spread of the distributions and the location of the IQR appear to correlate with the activating factors $\mathrm{PIP}_{2}$ and pseudophosphorylation. The IQR of fully activated ezrin, i.e., ezrin $\mathrm{T}_{5} 67 \mathrm{D}$ in presence of $\mathrm{PIP}_{2}$, is shifted the most to high $W_{\mathrm{ad}}$, norm. Remarkable is the situation for ezrin wildtype in presence of $\mathrm{PIP}_{2}$ as the IQR appears to combine the IQRs of both ezrin wildtype and ezrin $\mathrm{T}_{56} 6 \mathrm{D}$ in presence of $\mathrm{PIP}_{2}$. This is reflected in the respective histogram by a bimodal distribution (figure 6.18). We may speculate that a fraction of ezrin wildtype in presence of $\mathrm{PIP}_{2}$ exhibits similar adhesion characteristics as ezrin $\mathrm{T}_{5} 67 \mathrm{D}$ with $\mathrm{PIP}_{2}$, whereas another fraction behaves more or less like ezrin wildtype in absence of $\mathrm{PIP}_{2}$.

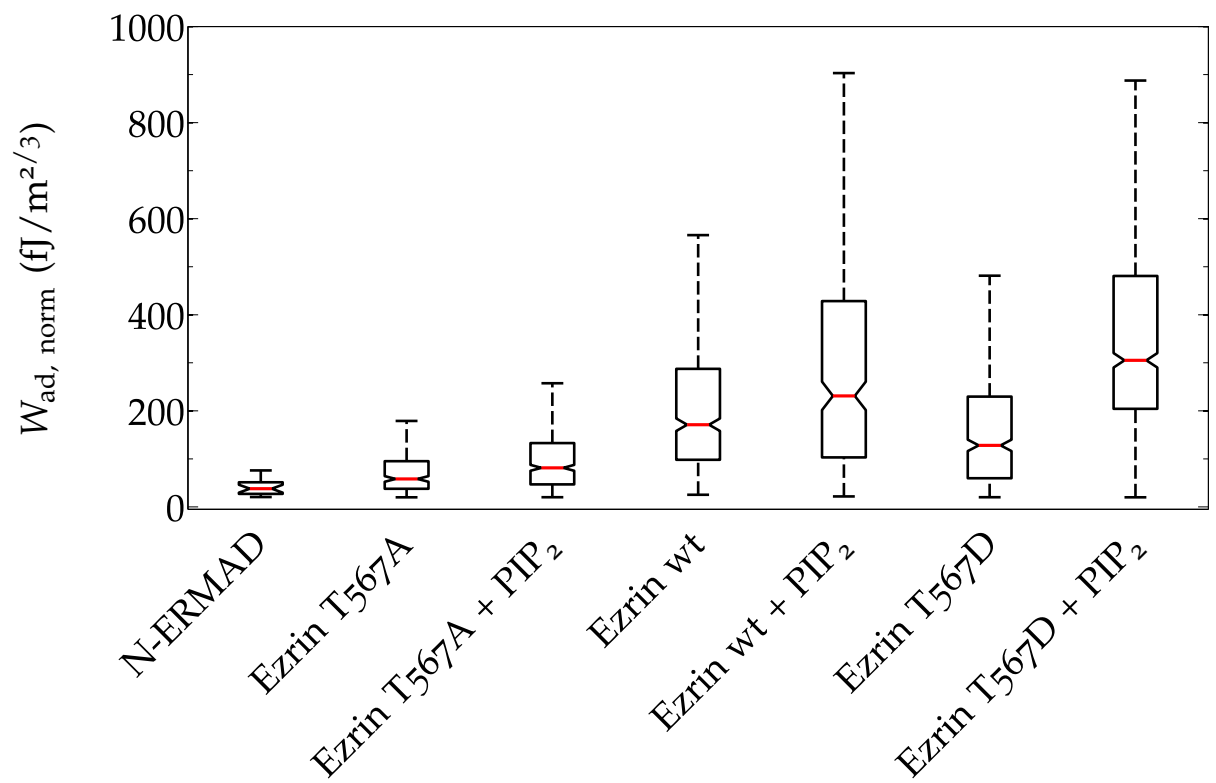

Figure 6.20: Box plot distribution of normalized surface adhesion energies resulting from the interaction between F-actin and ezrin depending on its activation. N-ERMAD is included as control. The red line represents the median, the solid box is the interquartile range (IQR) and the whiskers (dashed lines) are defined in length by $1.5 \times \mathrm{IQR}$. Data points outside this range are not shown. Notches indicate the location of the median at $95 \%$ confidence.

Based on the $W_{\mathrm{ad}}$, norm distribution of ezrin wildtype as well as ezrin $\mathrm{T}_{56} \mathrm{D} \mathrm{D}$ in presence of $\mathrm{PIP}_{2}$, we argue that this lipid is not only essential with regard to membrane anchoring, but also contributes significantly to the activation of ezrin in terms of rendering the F-actin binding site more accessible. Notably, a contribution of the pseudophosphorylation to higher $W_{\text {ad, norm }}$ values is only observable in combina- 
tion with $\mathrm{PIP}_{2}$, acting synergistically to enforce the interaction between ezrin and F-actin.

6.2.4 Low adhesion forces in CPM experiments - a comparison with single molecule experiments

Although we expected to probe many ezrin molecules simultaneously in the CPM experiments, we observed that the major part of the adhesion forces was below $100 \mathrm{pN}$, which is a reasonable range of single molecule rupturing. ${ }^{[219]}$ To evaluate the magnitude of the interaction between a single ezrin and F-actin, we investigated the interaction between F-actin and the pseudophosphorylated mutant ezrin $T_{56}{ }_{7} \mathrm{D}$ for comparison in single molecule force measurements (SMFM). The experimental design was chosen according to the CPM experiments (figure $6.4 \mathrm{~A}$ ). However, a Biolever was used instead of a colloidal probe. This cantilever is characterized by a very small tip radius of $\sim 30 \mathrm{~nm}$ to ensure that only one protein comes into contact with the sample surface in the ideal case. Figure 6.21 A shows a representative force distance curve exhibiting one single rupture event of $\sim 50 \mathrm{pN}$. The box plot distribution in figure $6.21 \mathrm{~B}$ reveals that the $F_{\text {ad }}$ values at similar loading rates $v_{F}$ are in the same range, indicating that the difference between ensemble (CPM) and single molecule measurement (SMFM) is not as pronounced as expected.

A

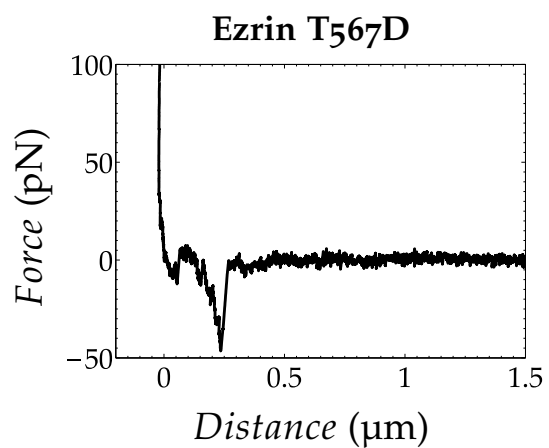

B

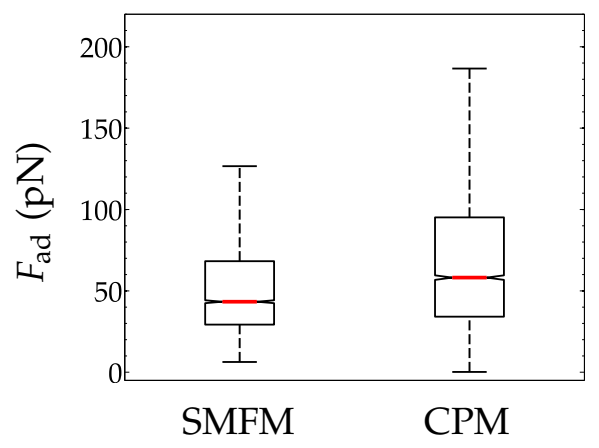

Figure 6.21: A Force distance curve showing single rupture events in SMFM experiment. B Box plots of adhesion forces $F_{\text {ad }}$ in SMFM and CPM experiments in comparison at similar loading rates $\left(v_{F} \sim 4 \times 10^{3} \mathrm{pN} / \mathrm{s}\right.$, calculated from the product of the effective stiffness (figure $6.28)$ and the pulling velocity).

With this setup in hand, we intended to characterize the interaction between ezrin and F-actin as a function of the applied loading rate by molecular force spectroscopy (MFS). 


\section{Loading rate dependence}

According to Bell's and Evan's theory (section 6.1), the adhesion forces should be shifted towards higher values upon increase of the loading rate $v_{F}$. More precisely, a linear dependence of the adhesion force on the logarithm of the loading rate $v_{F}$ is expected. The distribution of $F_{\mathrm{ad}}$ as a function of the natural logarithm of $v_{F}$ is depicted in figure 6.22. Loading rates in the range of $10^{2} \mathrm{pN} / \mathrm{s}$ to $7 \times 10^{4} \mathrm{pN} / \mathrm{s}$ were covered.

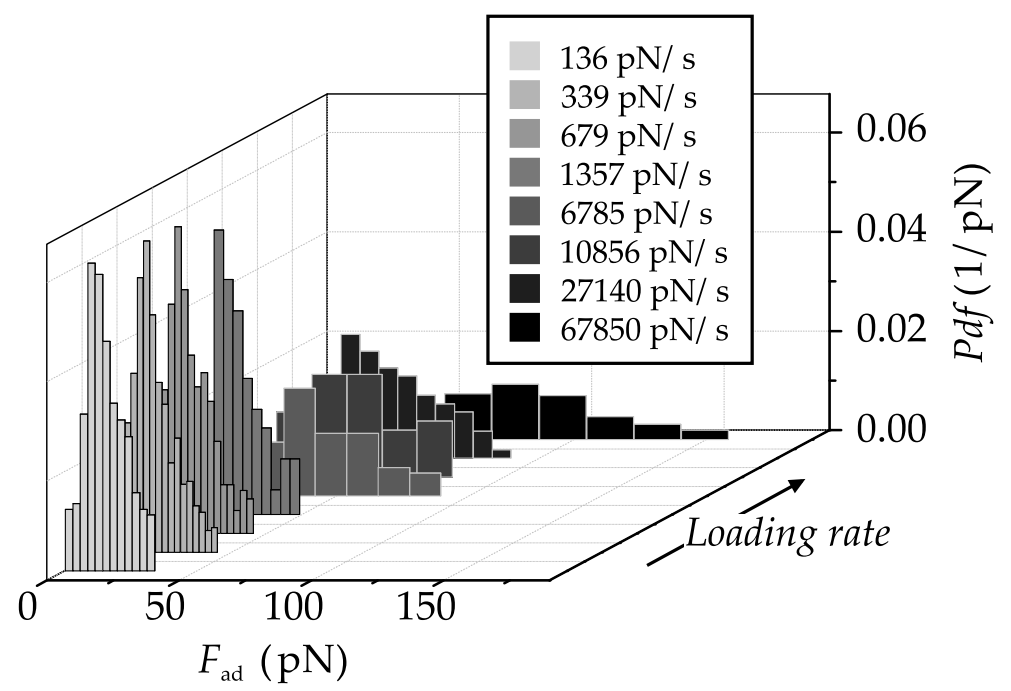

Figure 6.22: Molecular force spectroscopy probing the loading rate dependence of adhesion force $F_{\mathrm{ad}}$ between ezrin $\mathrm{T}_{567} \mathrm{D}$ and F-actin. The distribution of $F_{\mathrm{ad}}$ is visualized by histograms (different gray shades).

With increasing $v_{F}$ the distributions are shifted to higher $F_{\text {ad }}$ as well as broadened. At the lowest applied loading rate $\left(v_{F}=137 \mathrm{pN} / \mathrm{s}\right)$, the most probable adhesion force $F_{\mathrm{ad}}^{*}$ amounts to $12 \mathrm{pN}$, whereas at the fastest loading rate $\left(v_{F}=67850 \mathrm{pN} / \mathrm{s}\right)$ an increase to $75 \mathrm{pN}$ was observed. Similarly, the mean adhesion force $\left\langle F_{\mathrm{ad}}\right\rangle$ is shifted from $16 \mathrm{pN}$ to $71 \mathrm{pN}$.

To further evaluate the dependence of $F_{\text {ad }}$ on the variation in $v_{F}$, the mean adhesion force $\left\langle F_{\text {ad }}\right\rangle$ is plotted as a function of $v_{F}$ on a semi-logarithmic scale (figure $6.23 \mathrm{~A})$. A linear trend is observed between $10^{2} \mathrm{pN} / \mathrm{s}$ and $3 \times 10^{4} \mathrm{pN} / \mathrm{s}$. At first sight, the value of $F_{\mathrm{ad}}$ determined for $v_{F}=7 \times 10^{4} \mathrm{pN} / \mathrm{s}$ appears to be an outlier. However, this mean value is calculated from three independent experiments, thus invalidating the outlier assumption. Therefore, $F_{\mathrm{ad}}$ is obviously nonlinear within the range of applied $v_{F}$. 
A

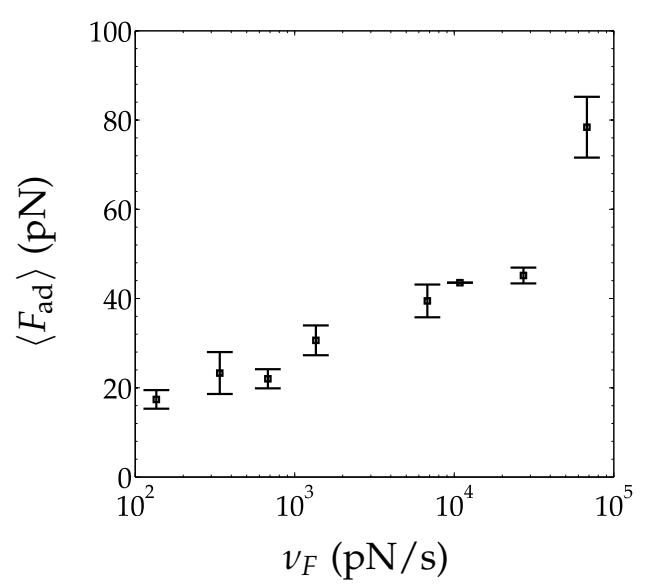

B

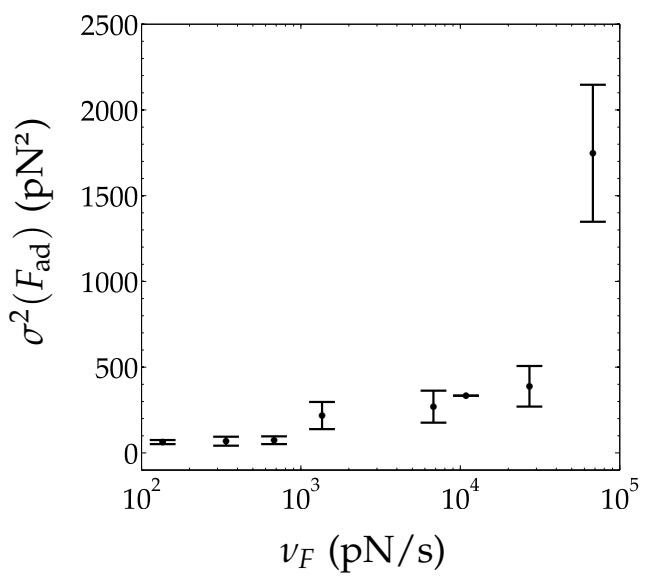

Figure 6.23: Loading rate dependence of mean adhesion force $\left\langle F_{\mathrm{ad}}\right\rangle$ between ezrin $\mathrm{T}_{567} \mathrm{D}$ and F-actin $(\mathbf{A})$ and variance $\sigma^{2}\left(F_{\mathrm{ad}}\right)(\mathbf{B})$. Mean values were determined for each applied $v_{\mathrm{F}}$. The corresponding error bars were calculated from standard deviation of the mean.

The variance $\sigma^{2}\left(F_{\mathrm{ad}}\right)$ is depicted in figure $6.23 \mathrm{~B}$. At $v_{F}<10^{3} \mathrm{pN} / \mathrm{s}$, the variance appears to be independent of $v_{F}$. Further increase in $v_{F}$, though, reveals that the variance is indeed a function of $v_{F}$.

\subsection{Discussion}

The aim of this work was to investigate quantitatively the interaction between Factin and ezrin by means of AFM to evaluate the influence of ezrin activation. An intramolecular head-to-tail association conformationally regulates the F-actin binding capability of ezrin. Dissociation and consequently release of the F-actin binding site thought to require $\mathrm{PIP}_{2}$ binding and phosphorylation of the threonine at position $567 \cdot{ }^{[1]}$ To investigate the individual contribution of the respective activation factor, we made use of ezrin wildtype, pseudophosphorylated and nonphosphorylatable ezrin mutants and submicellar PIP $_{2}$ as shown in table 6.1.

First, we will validate the functionalization strategy, while in the following we propose an answer to the question how the interaction between ezrin and F-actin is affected by the activation factors phosphorylation and $\mathrm{PIP}_{2}$ based on the CPM experiments. Thereafter, we reveal a rather surprising linkage between SMFM and CPM experiments and suggest a model for ezrin activation. The remainder of the discussion focuses on the force spectroscopic characterization of the bond between ezrin and F-actin. 


\subsubsection{NTA-Ni-His ${ }_{6}$ and electrostatics overcome the ezrin F-actin connection}

Crucial with regard to force measurements is that the interaction of interest is the weakest one, i.e., both NTA-Ni-His 6 -tag and F-actin-AUT ${ }^{+}$interaction are required to be stronger than the interaction between ezrin and F-actin. Janke et al. could show in CPM experiments that the cumulative work of adhesion between an Factin covered substrate and an F-actin coated colloid significantly exceeds the one between ezrin and F-actin. ${ }^{[15]}$ Furthermore, Verbelen et al. investigated the NTA$\mathrm{Ni}-\mathrm{His}_{6}$ bond in single molecule force measurements and found three maxima at rupture forces of $(153 \pm 57) \mathrm{pN},(316 \pm 50) \mathrm{pN}$, and $(468 \pm 44) \mathrm{pN}$ at a loading rate of $6600 \mathrm{pN} / \mathrm{s}$, thus exceeding the typical $F_{\mathrm{ad}}^{*}$ values for the interaction between ezrin and F-actin ranging from $26 \mathrm{pN}$ to $35 \mathrm{pN}$ in our experiments. ${ }^{[220]}$ Accordingly, the interaction of interest, i.e., the interaction between ezrin and F-actin, is the weakest one in the system and therefore expected to rupture first.

The functionalization strategy was validated by means of SPR and CLSM. We were able to show that the His $_{6}$-tagged protein was specifically bound to the NTANi-containing SAM, while the F-actin was firmly attached to a positively charged SAM via electrostatic interactions. In particular, the oriented $\mathrm{N}$-terminal immobilization of ezrin was essential to guarantee its specific interaction with F-actin via the $C$-terminal F-actin binding site. In a previous study, we could show that the $N$ terminal His $_{6}$-tag immobilization strategy is suitable with regard to specific F-actin adsorption. ${ }^{[14]}$ Moreover, high binding affinities of several $\sim 10 \mathrm{nM}$ were found for all three variants to either NTA-Ni or PIP ${ }_{2}{ }^{\left[{ }^{[221]}\right.}$ Therefore, we concluded that essentially the same protein density on the surface for ezrin wildtype, ezrin $\mathrm{T}_{5} 67 \mathrm{~A}$, and ezrin $\mathrm{T}_{567} \mathrm{D}$, respectively, was obtained, which is prerequisite for the comparison among the different interaction scenarios (table 6.1).

\subsubsection{Activation of ezrin: Increasing surface adhesion energies instead of adhesion forces}

The $F_{\mathrm{ad}}$ and $W_{\mathrm{ad}}$, norm values determined for the interaction between F-actin and ezrin in CPM experiments were evaluated by histogram analysis, empirical cumulative distributions, and box plots. We were able to show that all distributions of $F_{\text {ad }}$ differ from each other as such that they do not belong to the same distribution according to the two-dimensional KS test. The same result was obtained upon testing the distributions of $W_{\mathrm{ad}}$, norm .

The influence of ezrin activation on the $F_{\text {ad }}$ distribution was not as pronounced as expected. Although the distribution of $F_{\mathrm{ad}}$ appeared to change upon ezrin activation in general, the overall shift was too small to allow any conclusions with regard to an increased $F_{\mathrm{ad}}$ as a result of the activation process (figure 6.17). 
However, the $W_{\mathrm{ad} \text {, norm }}$ distributions showed clear differences regarding the different activation states (figure 6.20). Based on the high $W_{\mathrm{ad}}$, norm values found for ezrin wildtype as well as ezrin $\mathrm{T}_{56} 6 \mathrm{D}$ in presence of $\mathrm{PIP}_{2}$, we argue that this lipid is not only essential with regard to membrane anchoring, but also contributes significantly to the activation of ezrin in terms of rendering the F-actin binding site more accessible.

This pivotal role of $\mathrm{PIP}_{2}$ was reported by Janke et al. in CPM experiments, where they observed in presence of $\mathrm{PIP}_{2}$ a distinct increase in cumulative adhesion energy between ezrin, which was immobilized via PIP $_{2}$ at a solid-supported membrane, and an F-actin coated probe. ${ }^{[15]}$ However, they did not investigate the influence of phosphorylation. Similarly, we could show in a previous study that the F-actin surface coverage of $\mathrm{PIP}_{2}$ bound ezrin wildtype is increased more than twofold as compared to the one of NTA-Ni immobilized ezrin wildtype on solid-supported membranes. ${ }^{[14]}$ Carvalho et al. examined the changes in the intrinsic tryptophane fluorescence of ezrin wildtype and a mutant deficient in specific $\mathrm{PIP}_{2}$ binding. ${ }^{[176]}$ In presence of $\mathrm{PIP}_{2}$ concentrations in the micromolar range, they observed different quenching characteristic, which they attributed to a conformational change in case of the wildtype and to nonspecific interactions for the mutant. The involvement of $\mathrm{PIP}_{2}$ in the conformational change of ezrin is corroborated by Maniti et al., reporting a higher sensitivity of ezrin to digestion by chymotrypsin in presence of $\mathrm{PIP}_{2}$ thus concluding that a conformational change renders cleavages sites more accessible. ${ }^{[161]}$ The latter two studies as well as our work employ $\mathrm{PIP}_{2}$ in solution rather than incorporated in a membrane. This approach may be challenged from a cellular point of view as PIP $_{2}$ is rather incorporated in the membrane than dispersed in the cytosol. However, a similar quenching of the intrinsic tryptophane fluorescence was observed when ezrin was bound to $\mathrm{PIP}_{2}$-containing large unilamellar vesicles (LUVs). ${ }^{[161]}$

Notably, the pseudophosphorylation appears not to contribute on its own to an increased F-actin binding capability as the distribution of $W_{\mathrm{ad}}$, norm for ezrin $\mathrm{T}_{56}{ }_{7} \mathrm{D}$ is even shifted slightly to lower $W_{\mathrm{ad}}$, norm values as compared to ezrin wildtype. Conversely, we reported an F-actin surface coverage in case of NTA-Ni immobilized ezrin $\mathrm{T}_{567} \mathrm{D}$ that was similar to $\mathrm{PIP}_{2}$ bound ezrin wildtype, thus suggesting a partially active conformation of ezrin $\mathrm{T}_{56}{ }^{6}{ }_{\mathrm{D}}{ }^{\left[{ }^{[14}\right]}$ The influence of phosphorylation at this conserved threonine residue on ERM proteins is widely documented in literature. Several in vivo studies reported colocalization of such phosphorylated ERMs with actin-rich membrane protrusions. ${ }^{[222,223]}$ However, Matsui et al. reported similar F-actin binding capabilities in terms of the analogous cosedimentation of F-actin with either phosphorylated or non-phosphorylated C-terminal radixin. ${ }^{[9]}$ A corresponding behavior was observed by Huang et al. for $C$-terminal moesin, mimicking 
its phosphorylation by substitution of T558 with aspartate (T558D). Nevertheless, they could show that the pseudophosphorylation enhanced the cosedimentation of the full length moesin mutant with F-actin, indicating a partial release of the Factin binding site owing to electrostatic repulsion. ${ }^{[175]}$ Interestingly, Chambers et al. showed in binding assays that the $\mathrm{T}_{56} 6 \mathrm{D}$ mutation in ezrin impairs only to a minor extent the association of $\mathrm{N}$ - and C-termini. ${ }^{[173]}$ Jayasundar et al. corroborated this finding by using contrast variation small angular neutron scattering, showing that ezrin wildtype as well as ezrin $\mathrm{T}_{5} 67 \mathrm{D}$ adopt a closed conformation, i.e., no release of the F-actin binding site was detectable upon pseudophosphorylation. ${ }^{[174]}$

Regarding the similar spread of $W_{\mathrm{ad}}$, norm in case of ezrin wildtype and ezrin $\mathrm{T}_{567} \mathrm{D}$ both in presence of $\mathrm{PIP}_{2}$, we suggest that the pseudophosphorylation is not a prerequisite for efficient binding of ezrin to F-actin. This is in accordance with in vivo studies reporting that the phosphorylation is not necessarily essential with regard to the ability of ERMs to provide a linkage between plasma membrane and actin cytoskeleton. Yonemura et al. observed that ERM proteins appear to be activated in the absence of Rho induced phosphorylation and more importantly remain active, their lack of phosphorylation notwithstanding. ${ }^{[11]}$ Moreover, the authors could show that the microinjection of neomycin, a polyphosphoinositide $\left(\mathrm{PIP}_{2}\right)$ binding drug, impaired severely ERM activation regardless of Rho activity. Based on these results, they proposed that the activation of ERM proteins does not necessarily rely on phosphorylation but requires rather a local increase in $\mathrm{PIP}_{2}$ concentration, at least in certain cell types. Roch et al. analyzed the contribution of $\mathrm{PIP}_{2}$ binding and phosphorylation to the regulation of moesin during Drosophila development. ${ }^{[13]}$ They postulated a pool of open, non-phosphorylated moesin that is capable of establishing the connection to the actin cortex. Phosphorylation appeared to be required for proper morphogenesis depending on the type of tissue, however. Consistently, they proposed that ERM activity is initiated by $\mathrm{PIP}_{2}$ and further stabilized by phosphorylation. The authors suggested that the role of the phosphorylation is rather a fine-tuning mechanism, reinforcing the already established connection between plasma membrane and the F-actin cytoskeleton, thus providing additional control for the cell. In this framework, we may attribute the fact that the $W_{\mathrm{ad}}$,norm distribution of ezrin $\mathrm{T}_{56} 6 \mathrm{D}$ in presence of $\mathrm{PIP}_{2}$ is shifted to higher values as compared to that of ezrin wildtype with $\mathrm{PIP}_{2}$ to such fine-tuning characteristics.

Notably, the interaction involving ezrin $\mathrm{T}_{5} 67 \mathrm{~A}$ either with or without $\mathrm{PIP}_{2}$ is different. It appears that although the activating $\mathrm{PIP}_{2}$ is present, the substitution of threonine for alanine strongly hampers the F-actin binding capability of ezrin

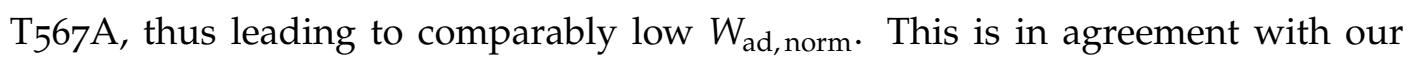
previous study, investigating the ezrin F-actin interaction by means of CLSM. ${ }^{[4]}$ 
In this study, we could show that independent of $\mathrm{PIP}_{2}$ binding the F-actin surface

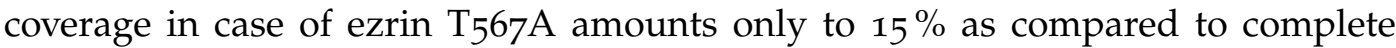
coverage in case of fully activated ezrin. Circular dichroism (CD) experiments further suggested that a structural change is caused by the substitution of threonine for alanine resulting in changed $\alpha$-helical as well as random coil contents compared to those determined for ezrin wildtype.

6.3.3 Ensemble versus single molecule measurement: Similar adhesion forces in both SMFM and CPM experiments

Although we expected to probe many ezrin molecules simultaneously, we observed mostly forces similar to that determined in SMFM below $100 \mathrm{pN}$, which is in a reasonable range of single molecule rupturing. ${ }^{[219]}$ Janke et al. employed a similar although reversed CPM assay to investigate the F-actin binding affinity of ezrin wildtype that was immobilized via $\mathrm{PIP}_{2}$ to a solid-supported membrane (SSM) with an F-actin coated colloidal probe. We refrained from attaching F-actin to the cantilever with regard to SMFM and MFS experiments as the attachment of a single rod-like macromolecule appears rather challenging. They reported that in presence of $\mathrm{PIP}_{2}$, the maximum adhesion force, i.e., the global minimum in a force distance curve, is shifted from $(0.45 \pm 0.15) \mathrm{nN}$ to $(1.32 \pm 0.44) \mathrm{nN} .{ }^{[15]}$ In contrast to these results, we did neither observe maximum adhesion forces in this range nor such distinct shift in $F_{\text {ad }}$. However, in spite of the minor shift in $F_{a d}$, we did observe a distinct increase in $W_{\mathrm{ad} \text {,norm }}$ upon PIP 2 binding for ezrin wildtype similar to Janke et al. and even more pronounced for ezrin $\mathrm{T}_{56} 6 \mathrm{D}$. Interestingly, the authors showed single ezrin-actin rupture events exhibiting a rupture force of approximately $200 \mathrm{pN}$. Considering that the force required to rupture at a comparable loading rate a single streptavidin-biotin bond - one of the high affinity bonds in nature - amounts to $\sim 150 \mathrm{pN},{ }^{[224]}$ it remains elusive how to evaluate such high unbinding forces for a single F-actin-ezrin bond in the cellular context, where both mechanical stability and dynamic properties are required. Although we indeed observed comparatively low $F_{\text {ad }}$ in both SMFM and CPM experiments, it is still not clear why no pronounced shared bond loading, i.e., the macroscopic rupture force is quantized in integer multiples of the single rupture force, was observed in case of CPM.

In general, one major advantage of CPM is that owing to the defined contact geometry - sphere versus flat surface - it is in principle possible to calculate the contact area. In the framework of the Hertz model, the contact area is dependent on the radius of the probe, the load force, and the reduced Young's modulus of the materials in contact. ${ }^{[209]}$ Approximating that the contact area is determined by the 
properties of the adsorbed protein material, we can calculate a contact radius of $\sim 60 \mathrm{~nm}$ according to equation 6.8 using a reduced Young's modulus $E_{\text {tot }}=4 \mathrm{MPa}$ and Poisson ratio $v=0.4$, a sphere radius of $3.5 \mu \mathrm{m}$, and an applied force load of $200 \mathrm{pN}$. ${ }^{[4]}$ The resulting contact area amounts to approximately $10000 \mathrm{~nm}^{2}$. Assuming that the area occupied by one ezrin is mainly determined by its $N$-terminal part, we are able to derive a required area of $\sim 30 \mathrm{~nm}^{2}$ per ezrin. ${ }^{[119]}$ Assuming random sequential adsorption of ezrin, a packing density of $55 \%$ is obtained. ${ }^{\text {[225] }}$ Hence, we can approximate 180 ezrin molecules located within the contact area under the assumption of a densely packed protein layer. Obviously, this is a rather rough estimate completely neglecting the adhesion of the sample. Nevertheless, it gives an impression on the magnitude of how many molecules should be involved in this interaction. Assuming that the colloid attached to the end of a cantilever is an ideal sphere with a smooth surface on the atomic level, we would expect approximately 180 interactions between ezrin proteins and F-actin to be formed (figure $6.24 \mathrm{~A}$ ). On this ground, the interaction forces should add up owing to shared bond loading, thus resulting in considerably larger $F_{\mathrm{ad}}$ in CPM than compared to those determined in SMFM experiments. However, as mentioned above we observed that $F_{\mathrm{ad}}$ is in a similar range for both CPM and SMFM. A possible explanation for this rather counterintuitive observation lies in the inherent surface roughness of the used colloidal probes. The colloids are made of pure gold and the SEM micrograph (figure 6.24 C) reveals that instead of a smooth surface we deal with microscopically rough features not to mention nanoscopic asperities, which are not resolved by SEM. Losic et al. found a root mean square roughness of $R_{\text {rms }}=$ $(5.1 \pm 0.5) \mathrm{nm}$ for bulk gold in AFM studies. ${ }^{[226]}$

A

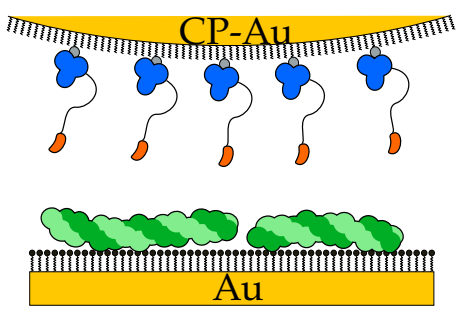

B

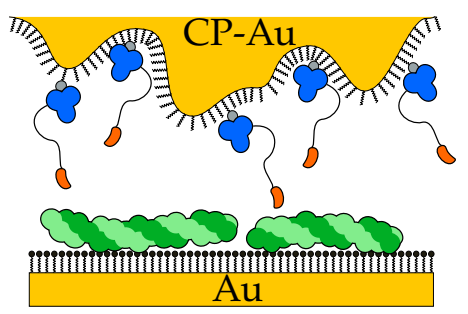

C

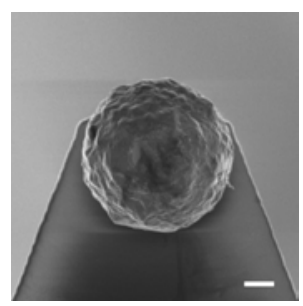

Figure 6.24: Schematic showing the influence of surface roughness on the interaction geometry in CPM: A Ideal colloidal probe sphere. B Rough colloidal sphere. C SEM micrograph showing a typical colloidal probe. Scale bar: $1 \mu \mathrm{m}$.

Therefore, the schematic $6.24 \mathrm{~A}$ is rather replaced by the one, depicted in figure $6.24 \mathrm{~B}$, providing a more realistic model. In this scenario, the number of interactions would be diminished to a great extent. Rabinovich et al. measured the interaction between a glass sphere and substrates, exhibiting different roughness profiles. ${ }^{[227]}$ They found that the adhesion force was reduced by $\sim 80 \%$ when the substrate, having a single roughness profile with $0.17 \mathrm{~nm}$, was replaced by one with 
two superimposed roughness profiles (1.64 $\mathrm{nm}$ and $10.5 \mathrm{~nm})$. At first, this might be a rather obvious explanation for our drastically reduced $F_{\text {ad }}$. One possibility to reduce the effect of surface roughness is the use of stiff cantilevers and the application of higher loads to cause flattening of nanoscale asperities. However, in case of solids such as gold, characterized by a Young's modulus of $78 \mathrm{GPa}$, it is rather unlikely to induce deformation upon loading with $200 \mathrm{pN} .^{[228]}$ We chose to apply such low force loads to avoid damaging the ezrin and F-actin layers. Exactly these layers, composed of soft biological material, however, will be deformed to a certain degree upon applied force, thus diminishing the impact of rough features.

Not only surface roughness but also heterogeneities in F-actin as well as ezrin coverage can affect the number of contact points. To reduce the impact of F-actin heterogeneity we carefully evaluated the degree in F-actin surface coverage by means of CLSM and used only comparable ones for the experiments, exhibiting an F-actin surface coverage of $(67 \pm 7) \%$. Furthermore, we applied the same NTA-Ni surface density in all measurements to ensure similar protein coverage. Moreover, sample heterogeneity is in particular an issue with conventional AFM cantilevers. However, we employed large spheres instead of sharp tips, thus averaging over a greater area and reducing the impact of sample heterogeneity on $F_{\text {ad }}$. In conclusion, we have factors that lead to a clearly reduced number of contact points including surface roughness and sample heterogeneity. But on the contrary, these effects are diminished as we use large spheres and samples coated with soft, deformable material. Therefore, these factors are most probable not the only ones leading to such small $F_{\text {ad }}$ values suggesting single bond rupture in the CPM experiments.

The distribution of $F_{\mathrm{ad}}$ is determined by the way the force is applied to load the formed bonds (figure 6.25). In CPM experiments, we expect to form a large number of parallel bonds as a comparably large colloid replaces the conventional sharp AFM tip. Accordingly, the applied force is equally shared among them and $F_{a d}$ is determined by the number of formed bonds (figure 6.25 A).

A

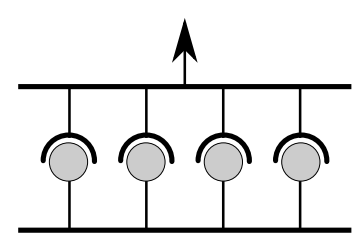

B

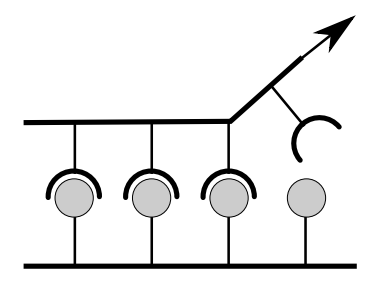

Figure 6.25: Different bond loading scenarios: parallel loading (A) and zipper mechanism leading to serial loading (B). The arrow illustrates the direction of the applied force. 
In contrast, serial loading is characterized by single bonds rupturing sequentially. For instance, the separation of DNA strands can be approximated by an unzipping mechanism as indicated in figure 6.25 B. ${ }^{[229,230]}$

Considering the three-dimensional case of the colloid being retracted from the surface, we might observe the sequential rupture of circularly arranged parallel bonds, i.e., the bonds at the rim of the contact zone are loaded whereas the ones located further toward the middle are not loaded until the outside bonds break. However, even under the assumption of such a loading scenario, we would still expect $F_{\text {ad }}$ being a function of several parallel bonds.

Figure 6.26 shows a spring model, where the elastic properties of cantilever and bonds are approximated by harmonic springs with stiffnesses $k_{\mathrm{c}}$ and $k_{\mathrm{s}}$, respectively, following Erdmann's work on the behavior of multiple bonds under loading. ${ }^{[231]}$

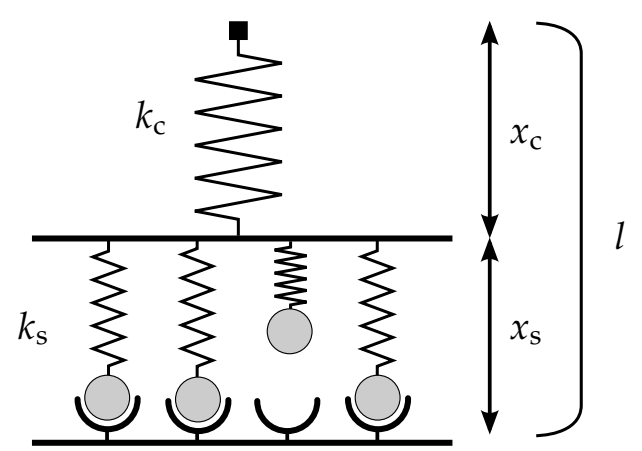

Figure 6.26: Spring model describing bond loading in colloidal probe microscopy. The stiffnesses of cantilever and bond are given by $k_{\mathrm{c}}$ and $k_{\mathrm{s}}$, respectively. The displacement $l$ denotes the distance between resting positions of transducer and bonds. It is determined by extensions of bond $x_{\mathrm{s}}$ and cantilever $x_{\mathrm{c}}$.

If $N$ bonds are closed, they are able to sustain force. Hence, a displacement $l>0$ causes an extension of both cantilever spring $x_{\mathrm{c}}$ and bond springs $x_{\mathrm{s}}$. According to Hooke's law, the required forces $F_{\mathrm{c}}$ and $F_{\mathrm{s}}$ to extend the cantilever and a single bond, respectively, are given by:

$$
F_{\mathrm{c}}=k_{\mathrm{c}} x_{\mathrm{c}} \quad \text { and } \quad F_{\mathrm{s}}=k_{\mathrm{s}} x_{\mathrm{s}} .
$$

Considering that the displacement $l$ is given by:

$$
l=x_{\mathrm{c}}+x_{\mathrm{s}}
$$

the balance of force between cantilever and $N$ closed bonds satisfies:

$$
F_{\mathrm{c}}=k_{\mathrm{c}} x_{\mathrm{c}}=k_{\mathrm{c}}\left(l-x_{\mathrm{s}}\right)=N k_{\mathrm{s}}\left(l-x_{\mathrm{c}}\right)=N k_{\mathrm{s}} x_{\mathrm{s}}=N F_{\mathrm{s}} .
$$


This equation allows to derive expressions for the respective extensions as a function of $l$ and $N$. This is pivotal considering that rather the displacement $l$ than the actual force is controlled in most force experiments:

$$
x_{\mathrm{c}}=\frac{N k_{\mathrm{s}} l}{k_{\mathrm{c}}+N k_{\mathrm{s}}} \quad \text { and } \quad x_{\mathrm{s}}=\frac{k_{\mathrm{c}} l}{k_{\mathrm{c}}+N k_{\mathrm{s}}} .
$$

Substituting $x_{\mathrm{c}}$ or $x_{\mathrm{s}}$ in equation 6.12 with the corresponding expression derived in equation 6.13, the balance of force depending on $l$ is given by:

$$
F_{\mathrm{c}}=k_{\mathrm{c}} x_{\mathrm{c}}=\frac{N k_{\mathrm{c}} k_{\mathrm{s}} l}{k_{\mathrm{c}}+N k_{\mathrm{s}}}=N k_{\mathrm{s}} x_{\mathrm{s}}=N F_{\mathrm{s}}
$$

This relation shows that $F_{\mathrm{c}}$ is determined by the ensemble of $N$ bonds with an effective stiffness $k_{\text {eff }}$ :

$$
k_{\mathrm{eff}}=\frac{N k_{\mathrm{c}} k_{\mathrm{s}}}{k_{\mathrm{c}}+N k_{\mathrm{s}}}
$$

The force per bond is given by:

$$
F_{\mathrm{s}}=\frac{k_{\mathrm{eff}}}{N} l
$$

Consequently, we would expect to observe $N$ times higher adhesion forces in CPM than SMFM experiments. Notably, Seifert showed in his deterministic model that this intuitive expectation is strongly dependent on the stiffness ratio of $k_{\mathrm{c}}$ to $k_{\mathrm{s}}$ in case of force experiments controlling the displacement instead of force. ${ }^{[232]} \mathrm{He}$ derived two limiting cases, namely the soft transducer, which is characterized by shared bond loading, and the stiff transducer, exhibiting only weak dependence of adhesion forces on the number of bonds. The limit of a soft transducer case is given by $k_{\mathrm{s}} \gg k_{\mathrm{c}}$ (figure $6.27 \mathrm{~A}$ ).

A

$$
k_{\mathrm{s}} \gg k_{\mathrm{c}}
$$

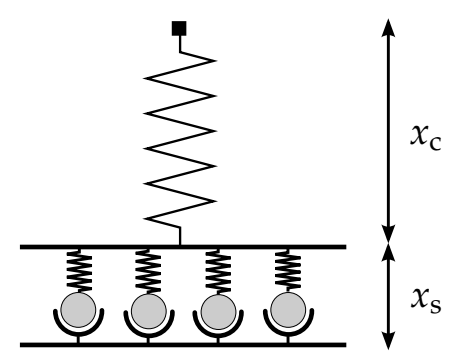

B

$$
N k_{\mathrm{s}} \ll k_{\mathrm{c}}
$$

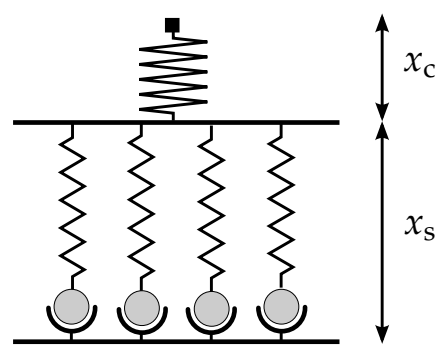

Figure 6.27: Spring model showing shared loading (A), where the cantilever stiffness $k_{\mathrm{c}}$ is much less than the stiffness of a single bond $k_{\mathrm{s}}$. The cantilever extension $x_{\mathrm{c}}$ is much greater than the extension $x_{\mathrm{s}}$ of the bonds. Contrary to this behavior is the case of non-cooperative loading $(\mathbf{B})$, where the overall bond stiffness $N k_{\mathrm{s}}$ is much less than cantilever stiffness $k_{\mathrm{C}}$, resulting in $x_{\mathrm{c}} \ll x_{\mathrm{s}}$. 
That is when multiple parallel bonds are loaded by a very soft transducer or cantilever, its extension $x_{\mathrm{c}}$ is much greater than the extension of the bonds $x_{\mathrm{s}}$. Consequently, the displacement $l$ is mainly determined by $x_{c}$, simplifying equation 6.12 to:

$$
F_{\mathrm{c}}=k_{\mathrm{c}} l
$$

while the force per bond $F_{\mathrm{s}}$ is determined by $N$ closed bonds with:

$$
F_{\mathrm{s}}=\frac{F_{\mathrm{c}}}{N}=\frac{k_{\mathrm{c}} l}{N}
$$

This is the case of shared loading, where the coupling among closed bonds is most prominent.

In contrast, the limit of a stiff transducer, where $k_{\mathrm{c}} \gg N k_{\mathrm{s}}$, is shown in figure 6.27 B. In this case, the loading of bonds leads to their extension, determining $l$, whereas the extension of the cantilever spring is neglectable due to its high stiffness. The force per bond $F_{\mathrm{S}}$ is now directly controlled by $l$. Consequently, equation 6.12 simplifies to:

$$
F_{\mathrm{s}}=k_{\mathrm{s}} l
$$

while the force acting on the cantilever is dependent on $N$ :

$$
F_{\mathrm{c}}=N F_{\mathrm{s}}=N k_{\mathrm{s}} l
$$

This non-cooperative loading is initially counterintuitive as although many bonds are probed simultaneously, only the force value of a single bond is detected. Following this interpretation, Lorenz et al. could show that the rupture forces of an adhesion cluster do not linearly increase with its size in case of rather soft bond stiffness. ${ }^{[233]}$

The system we probe in our force measurements is composed of F-actin, ezrin, thiol linker, and cantilever. The mechanical properties of the system are approximated by an effective spring constant $k_{\text {eff }}$ comprising the stiffnesses $k_{\mathrm{c}}$ and $k_{\mathrm{s}}$ of both cantilever and bond between ezrin and F-actin according to equation 6.15. Considering the serial compliance of cantilever and $N$ parallel bonds (figure 6.26), $k_{\text {eff }}$ is essentially determined by the weakest individual contribution:

$$
\frac{1}{k_{\mathrm{eff}}}=\frac{1}{k_{\mathrm{c}}}+\frac{1}{N k_{\mathrm{s}}}
$$

This equation is a transformation of equation 6.15, illustrating that the magnitude of $k_{\text {eff }}$ is mainly determined by the softest stiffness contribution. Values for $k_{\text {eff }}$ 
were obtained from linear fits to the part of the force distance curve preceding the rupture event for both SMFM and CPM (figure 6.28). The most probable effective stiffness $k_{\mathrm{eff}}^{*}$ amounts to $2.2 \mathrm{pN} / \mathrm{nm}$ and $1.4 \mathrm{pN} / \mathrm{nm}$ for CPM experiments and SMFM, respectively.

A

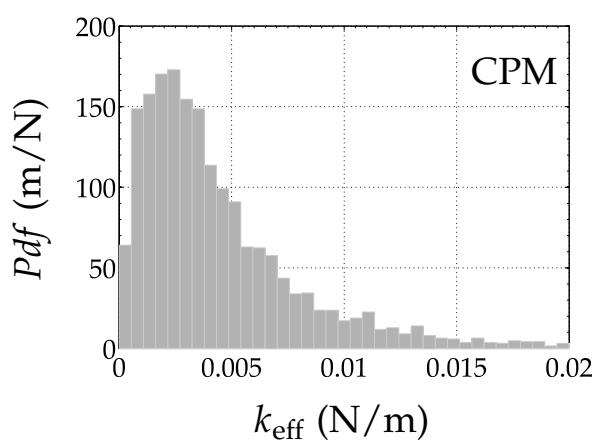

B

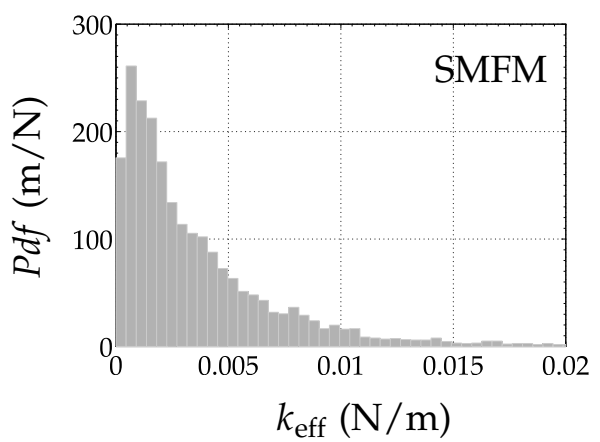

Figure 6.28: Effective stiffness $k_{\text {eff }}$ determined for colloidal probe microscopy (A) and single molecule force measurements (B).

We conclude for CPM experiments that $k_{\text {eff }}$ is essentially determined by the stiffness $N k_{\mathrm{s}}$ of the interaction between ezrin and F-actin as it is rather low compared to the stiffness of a colloidal probe $\left(k_{\mathrm{c}} \sim 40 \mathrm{pN} / \mathrm{nm}\right)$. If we assume $N k_{\mathrm{s}} \ll k_{\mathrm{c}}$, equation 6.15 simplifies to:

$$
k_{\mathrm{eff}} \approx N k_{\mathrm{s}}
$$

Within the framework of Seifert's and Erdmann's model, Lorenz et al. used an approach to estimate the energy landscape and the number of participating bonds of a small adhesion cluster within the contact area of a colloidal probe in the limit of a stiff transducer. ${ }^{[233]}$ The number of bonds $N$ was estimated from the overall mean molecular stiffness $\left\langle N k_{\mathrm{s}}\right\rangle$ and its variance $\sigma^{2}$ assuming a Poisson distribution satisfying the identity:

$$
k_{\mathrm{s}}=\frac{\sigma^{2}}{\left\langle N k_{\mathrm{s}}\right\rangle} .
$$

We calculated $\left\langle k_{\text {eff }}\right\rangle \approx\left\langle N k_{\mathrm{s}}\right\rangle$ from $>3400$ events (figure 6.28 A) and according to equation $6.23 k_{\mathrm{s}}$ is $4.6 \mathrm{pN} / \mathrm{nm}$ and $N$ amounts to $\sim 1$ in the CPM experiment. As we assume that $N k_{\mathrm{s}}$ determines $k_{\text {eff }}$, this result is in good agreement with the similar range of $k_{\text {eff }}$ observed in SMFM (6.28 B), where only a single bond is probed.

In conclusion, we postulate that our CPM measurements approximate the limiting case of a stiff transducer. To test this hypothesis, it would be most interesting to employ softer colloidal probes to determine whether the loading situation is shifted to shared loading resulting in considerable larger $F_{\mathrm{ad}}$. Admittedly, this is limited as such that a certain cantilever stiffness is required to withstand the gravity of an ad- 
ditional mass, i.e., the colloid, at its end. Janke et al. used four times softer colloidal probes as in our case and observed considerably higher $F_{\text {ad }}$ values, indicating that the loading situation is shifted to shared loading. ${ }^{\left[{ }^{[5]}\right.}$ Although we apparently probe single molecules in our CPM assay, we can still assess the number of formed bonds at least qualitatively evaluating $W_{\mathrm{ad}}$,norm. Its magnitude is determined as such that as soon as one bond fails, the next one is loaded subsequently, thus cumulating the more bonds are formed.

\subsubsection{Molecular force spectroscopy: Dependence of mean adhesion force on loading rate}

In the framework of the phenomenological approach, MFS experiments allow to extract the strength, lifetime as well as details of the energy landscape of an intermolecular interaction (section 6.1). The Bell-Evans model postulates for a single bond in the nonequilibrium a linear dependence of the observed rupture force on the logarithm of the loading rate, whereas the variance remains unchanged. As can be concluded from figure $6.23 \mathrm{~B}$, clear deviations from this prediction were observed. This is in agreement with an increasing number of MFS studies reporting an apparent nonlinearity in the plot of $F_{\text {ad }}^{*}$ versus $\ln \left(v_{F}\right)$ instead of a single linear regime. ${ }^{[234]}$ This behavior was attributed, however, to the presence of multiple barriers in the energy landscape and accordingly treated by applying straight line fits with different slopes. The seminal work of Merkel et al. investigating the biotin-avidin bond was to our knowledge the first reporting such deviations. ${ }^{[204]}$ Within the context of the phenomenological model, they concluded the existence of subsequent barriers along the rupture pathway, thus corroborating the exceptional strength of this interaction. The authors suggested the existence of three barriers, located at $x^{\ddagger}=1.2 \AA, 3 \AA$, and $30 \AA$. The assumption of multiple barriers has become popular within the field of MFS; in particular as rupture processes revealed frequently nonlinear trends, when investigated over a broad range of loading rates. Rather different types of interactions including peptides, ${ }^{[235]}$ macromolecules, ${ }^{[236]}$ and adhesion between silicon nitride cantilevers and hydrophobic SAMs ${ }^{[237]}$ have been reported to be characterized by multiple barriers.

The question whether the multi-barrier assumption adequately describes the energy landscape or if the apparent distinct linear regimes arise owing to limitations of the Bell-Evans model is a subject of controversy. However, the model relies on the assumption that the position of the transition state, i.e. $x^{\ddagger}$, remains unchanged with increasing $v_{F}$, leading to the prediction that the variance $\sigma_{F}^{2}$ is basically independent of $v_{F}$. This implied independence contradicts the observed increase in

$\sigma_{F}^{2}$ in both our experiments and simulation upon increasing $\nu_{F \cdot}{ }^{[238]}$ Therefore, we refrained from interpreting the nonlinearity in the plot of $F_{\mathrm{ad}} v s \ln v_{F}$ as evidence 
for a second energy barrier. Consequently, the Bell-Evans approach is only valid up to $v_{F} \sim 3 \times 10^{4} \mathrm{pN} / \mathrm{s}$ for our data.

The linear fit to the data is shown in figure 6.29. From the linear equation the potential width $x^{\ddagger}$ and the unbinding rate at zero force $k_{\text {off }}^{0}$ can be extracted. According to equation $6.2, x^{\ddagger}$ amounts to $7.2 \AA$, while $k_{\text {off }}^{0}$ is $\sim 1.3 \mathrm{~s}^{-1}$ at $T=294 \mathrm{~K}$.

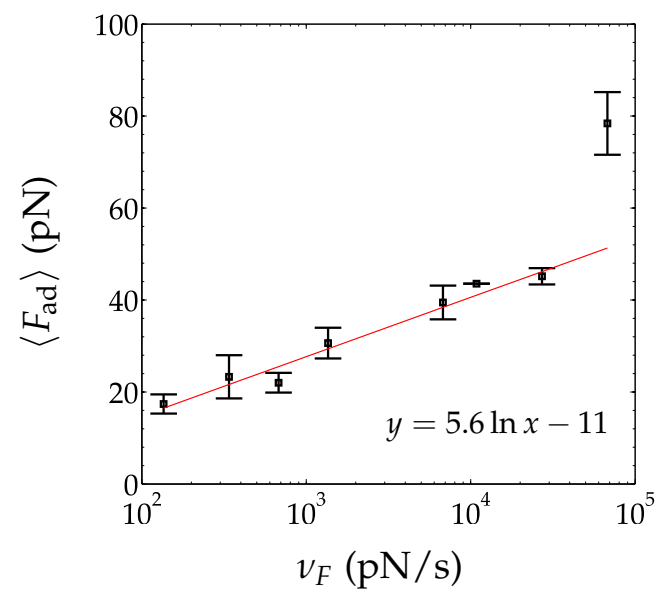

Figure 6.29: Loading rate dependence of mean adhesion force $\left\langle F_{\mathrm{ad}}\right\rangle$ between ezrin $\mathrm{T}_{567} \mathrm{D}$ and F-actin. Error bars were calculated from standard deviations. A straight line fit (red) yields $x^{\ddagger}$ and $k_{\text {off }}^{0}$ according to equation 6.2.

The values we obtained from the Bell-Evans model are in the range of typical values obtained in MFS experiments. ${ }^{[239]}$ Ferrer et al. determined for the interaction between F-actin and $\alpha$-actinin, which is an actin filament crosslinker, a potential width $x^{\ddagger}$ of $\sim 2.8 \AA$ and an intrinsic dissociation rate $k_{\text {off }}^{0}$ of $\sim 0.07 \mathrm{~s}^{-1}$. ${ }^{[240]}$ The comparatively high dissociation rate of $\sim 1 \mathrm{~s}^{-1}$ in our case implicates that the interaction between ezrin and F-actin is rather weak. However, $k_{\text {off }}^{0}$ values determined from mechanical unbinding experiments are in general not in close agreement with that determined in bulk measurements. ${ }^{[241]}$ Even for the same ligand-receptor system a large variety in $k_{\text {off }}^{0}$ can be found, e.g. for streptavidin/avidin, values ranging from $1 \times 10^{-5} \mathrm{~s}^{-1}$ to $30 \mathrm{~s}^{-1}$ were reported in different MFS studies. ${ }^{[239]}$

Other models than the Bell-Evans approach have to be used to describe the data obtained at $v_{F}<3 \times 10^{4} \mathrm{pN} / \mathrm{s}$. However, the attempt to use the microscopic model according to the work of Hummer and Szabo to account for the observed nonlinearity in the plot $F_{\text {ad }} v s \ln v_{F}$ and the found dependence of $\sigma^{2}$ on $v_{F}$ was not successful as this model did not describe our data adequately in the range of applied loading rates. ${ }^{[238]}$

\subsection{THE FORCE RESPONSE TO EZRIN ACTIVATION - CONCLUSION}

We showed that the activation of ezrin via $\mathrm{PIP}_{2}$ and pseudophosphorylation in our CPM assay involves a pronounced increase in normalized surface adhesion energy, 
indicating a strong interaction between activated ezrin and F-actin. Moreover, we have shown the necessity of $\mathrm{PIP}_{2}$ and further fine-tuning via phosphorylation to establish a tight connection. Notably, we were able to derive a relation between SMFM and CPM experiments accounting for the similar adhesion forces $<100 \mathrm{pN}$. We have shown, that single events can be detected in CPM experiments although an ensemble is probed. Moreover, CPM allows the determination of an overall surface adhesion energy resulting from many individual interactions. Strikingly, the activation of ezrin does not result in a pronounced increase in adhesion force. At first glance, this is rather unexpected. However, if we consider that ezrin is highly concentrated in microvilli, ${ }^{[148]}$ the necessity of a drastic increase in adhesion force of a single interaction is questionable. A strong individual interaction between ezrin and F-actin would probably hamper the dynamic properties of a cell. Therefore, we conclude that the number of ezrin molecules, that are capable of bind F-actin efficiently, increases upon the synergistic influence of $\mathrm{PIP}_{2}$ and phosphorylation rather than the interaction force (figure 6.30).

A

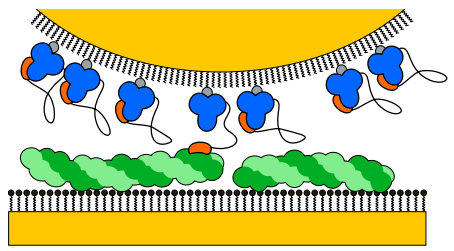

B

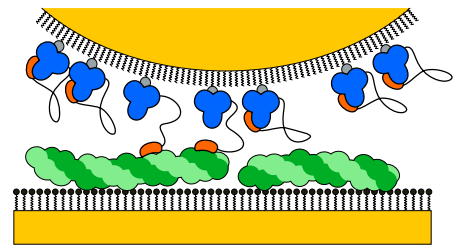

C

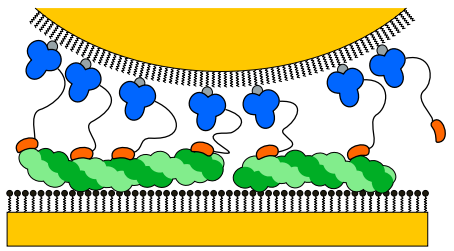

Figure 6.30: Schematic showing effect of ezrin activation: Ezrin is mostly inactive (A). Partial (B) and full activation (C) lead to increasing interaction with F-actin.

The principle of combining many weak bonds to form stable, intermolecular connections is used within the cellular context to allow for dynamic response to external or internal stimuli, whereas strong bonds are suited for static connections. ${ }^{\text {[242] }}$ In light of this and the molecular dimensions - nanometer sized ezrin versus micrometer long F-actin - an ezrin-F-actin connection formed by many individual ezrin molecules appears reasonable.

\subsection{CO-WORKERS}

Mey, Ingo

\subsection{EXPERIMENTAL DETAILS}

ACTIN POLYMERIZATION Lypophilized non-muscular actin ( $>95 \%$ pure, purified from human platelet) with an isotype composition of $85 \% \beta$-actin and $15 \%$ $\gamma$-actin was purchased from Cytoskeleton (Denver, USA). Reconstitution in $5 \mathrm{~mm}$ 
TRIS/ $\mathrm{HCl}, 0.2 \mathrm{~mm} \mathrm{CaCl}_{2}, 0.2 \mathrm{~mm}$ ATP, $5 \%$ sucrose $(w / v), 1 \%$ dextran $(w / v)$ at $\mathrm{pH} 8$ was achieved by addition of ultra pure water to a concentration of $10 \mathrm{mg} / \mathrm{ml}$. Aliquots of $10 \mu \mathrm{l}$ were snap-frozen in liquid nitrogen and stored at $-80^{\circ} \mathrm{C}$. Before use, the aliquot was diluted with cold G-buffer $\left(5 \mathrm{~mm}\right.$ TRIS/ $\mathrm{HCl}, 0.2 \mathrm{mM} \mathrm{CaCl}{ }_{2}$, $0.5 \mathrm{~mm}$ DTT, $0.2 \mathrm{~mm}$ ATP, $0.8 \mathrm{~mm} \mathrm{NaN}_{3}$ at $\mathrm{pH}_{7.4}$ ) to a concentration of $0.4 \mathrm{mg} / \mathrm{ml}$ and incubated on ice for $1 \mathrm{~h}$. Then, the actin solution was clarified by centrifugation at $17000 \mathrm{xg}$ for $15 \mathrm{~min}$ at $4{ }^{\circ} \mathrm{C}$ to remove remaining oligomers. The supernatant was transferred into a fresh Eppendorf tube and actin polymerization was induced by adding $1 / 10$ volume of polymerization solution $(500 \mathrm{mM} \mathrm{KCl}, 20 \mathrm{~mm}$ $\left.\mathrm{MgCl}_{2}, 20 \mathrm{~mm} \mathrm{ATP}\right)$. After $15 \mathrm{~min}, 1 \mathrm{~mol} \%$ of AlexaFluor488-phalloidin was added to fluorescently label actin filaments. After another $5 \mathrm{~min}$, an equimolar amount of phalloidin (with respect to monomeric actin) was added to further stabilize the formed filaments. Phalloidin is known to bind preferably to F-actin rather than to monomeric actin, thus shifting the equilibrium between filaments and monomers towards the filamentous form. ${ }^{[213]}$ The solution was left on ice for at least $1 \mathrm{~h}$ to ensure complete binding of phalloidin. Half of the resulting F-actin solution was added to an $\mathrm{AUT}^{+}$-functionalized gold coated substrate (paragraph 3.2.1.1) immersed in F buffer (20 mM TRIS/ $\mathrm{HCl}, 50 \mathrm{~mm} \mathrm{KCl}, 0.2 \mathrm{~mm} \mathrm{MgCl}_{2}$, 0.1 mM EDTA, O.I $\mathrm{mM} \mathrm{NaN}_{3}$ at $\mathrm{pH} 7.4$ ) and incubated overnight at $4{ }^{\circ} \mathrm{C}$.

CANTILEVER FUNCTIONALizATiON For CPM experiments, colloidal probes with attached gold colloids (diameter: $5.5 \mu \mathrm{m}$ to $9.5 \mu \mathrm{m}$ ) and a nominal spring constant of $0.08 \mathrm{~N} / \mathrm{m}$ were purchased from sQube (Bickenbach, Germany). For MFS and SMFM experiments, Biolever (Olympus, Tokyo, Japan) with a nominal spring constant of $0.006 \mathrm{~N} / \mathrm{m}$ were used. The spring constant was calibrated before each experiment as described in paragraph 3.3.1. The cantilevers were thiol functionalized as outlined in paragraph 3.2.1.1. An incubation step of $30 \mathrm{~min}$ in $\mathrm{NiCl}_{2}$ (100 mM, pH 8.o adjusted with TRIS) was performed to charge the NTA moieties with $\mathrm{Ni}^{2+}$. After copious rinsing with ultrapure water and E1 buffer, the cantilever was mounted into the standard holder provided by Asylum Research (Santa Barbara, USA). Protein binding was achieved by putting the holder on top of a flowthrough chamber containing $1 \mu \mathrm{M}$ protein solution. In case of experiments involving $\mathrm{PIP}_{2}$ activation, $1 \mu \mathrm{M}$ protein solution was pre-incubated for $30 \mathrm{~min}$ with $1 \mu \mathrm{M}$ submicellar $\mathrm{PIP}_{2}$ solution in E1 buffer by using a rotator. After $1 \mathrm{~h}$ flow-through protein incubation, the cantilever holder was removed, carefully rinsed with buffer, and mounted onto the AFM head.

FORCE MEASUREMENTS Before each experiment, the system was thermally equilibrated for $20 \mathrm{~min}$. During a force measurement, the protein covered probe 
was brought into contact with the F-actin functionalized surface (figure 6.4). Forcedistance cycles were performed at $200 \mathrm{pN}$ load with varying pulling velocities and matching sample rates. The dwell time was set to $1 \mathrm{~s}$. All measurements were performed in F buffer. Colloidal probes were controlled afterwards to ensure on the one hand that the colloid was still firmly attached. On the other hand, epifluorescence was used to ensure that no F-actin was adhered to the colloid. 

This work aimed to investigate in vitro pivotal components regulating the membranecytoskeleton interface, namely the membrane lipid L- $\alpha$-phosphatidylinositol-4,5-bisphosphate $\left(\mathrm{PIP}_{2}\right)$, the membrane-cytoskeleton linker ezrin, and filamentous actin (F-actin) at different levels. The function of ezrin is conformationally regulated by self-association of $\mathrm{N}$ - and $\mathrm{C}$-terminal domains, which is thought to dissociate upon binding of ezrin to $\mathrm{PIP}_{2}$ and subsequent phosphorylation of a specific threonine residue. The main focus of this work was to examine this activation process of ezrin considering the individual influence of $\mathrm{PIP}_{2}$ binding and phosphorylation on the conformational change and accumulation of ezrin on solid-supported membranes as well as the F-actin binding capability of ezrin depending on its activation state.

The first part of this thesis dealt with the characterization of $\mathrm{PIP}_{2}$-containing solid-supported lipid bilayers (SLBs) and hybrid membranes (SHMs, chapter 4). A profound influence of spreading conditions on the $\mathrm{PIP}_{2}$ distribution and accessibility in terms of ezrin binding was revealed in reflectometric interference spectroscopy (RIfS) and confocal laser scanning microscopy (CLSM) experiments. The use of either $200 \mathrm{~mm} \mathrm{NaCl}$ or $2 \mathrm{mM} \mathrm{Ca}^{2+}$ to promote spreading of small unilamellar vesicles containing up to $8 \mathrm{~mol} \% \mathrm{PIP}_{2}$ led to inhomogeneously distributed $\mathrm{PIP}_{2}$ and adhering excess vesicles in case of $\mathrm{Ca}^{2+}$. Consequently, such SLBs are not well suited for protein binding studies in combination with surface sensitive techniques such as atomic force microscopy (AFM) and RIfS. However, spreading at $\mathrm{pH} 4.8$ yielded SLBs characterized by homogeneously distributed $\mathrm{PIP}_{2}$ and no adhering excess vesicles, thus allowing the specific binding of ezrin. Moreover, it was shown that $\mathrm{PIP}_{2}$-containing SHMs exhibited a diffusion coefficient of $3 \mu^{2} / \mathrm{s}$ and a mobile fraction of $98 \%$ in fluorescence recovery after photobleaching (FRAP) experiments using a fluorescent $\mathrm{PIP}_{2}$ analogue as tracer. $\mathrm{Ca}^{2+}$ induced clustering of $\mathrm{PIP}_{2}$ led to a reduced diffusion coefficient of $1.4 \mu \mathrm{m}^{2} / \mathrm{s}$, whereas ezrin binding diminished the lateral mobility of $\mathrm{PIP}_{2}$ even further to $D=0.7 \mu \mathrm{m}^{2} / \mathrm{s}$. Consequently, ezrin might be able to maintain $\mathrm{PIP}_{2}$ clusters within the cellular context, which are thought to have an important role with regard to the functional versatility of PIP $_{2}$.

In the second part, the question was addressed whether the conformational change of ezrin due to activation correlates with changed height levels of ezrin 
aggregates on SLBs (chapter 5). Ezrin wildtype, nonphosphorylatable ezrin $\mathrm{T}_{56} 6 \mathrm{~A}$, and pseudophosphorylated ezrin $\mathrm{T}_{56} 6_{7} \mathrm{D}$ were each immobilized either to $\mathrm{PIP}_{2}-$ containing SLBs or via an $N$-terminal $6 \times$ histidine $\left(\mathrm{His}_{6}\right)$ tag to SLBs displaying nickel nitrilotriacetic acid (NTA-Ni) head groups. No substantially changed height level owing to phosphorylation alone could be detected in RIfS experiments. However, a clear decrease in height was observed in case of the combination of $\mathrm{PIP}_{2}$ binding and phosphorylation. This finding was confirmed by AFM imaging, where ezrin wildtype on $\mathrm{PIP}_{2}$-containing SLBs exhibited $3.5 \mathrm{~nm}$ height levels in contrast to the height value of $1.5 \mathrm{~nm}$ found for ezrin $\mathrm{T}_{567} \mathrm{D}$ on the same membrane system. The activating influence of $\mathrm{PIP}_{2}$ alone could not be unambiguously resolved by analyzing the height levels of ezrin wildtype on SLBs. However, activation of ezrin might be not only restricted to a dissociation of $\mathrm{N}$ - and $\mathrm{C}$-terminal domains leading to lower protein height levels, but also include the formation of a dense protein layer via lateral protein-protein interactions to offer as many binding site as possible toward F-actin. Binding to $\mathrm{PIP}_{2}$ might favor lateral protein-protein interaction leading to higher protein surface coverage for ezrin wildtype as compared to its immobilization to NTA-Ni head groups. Accordingly, the combination of evaluating the protein surface coverage and analyzing protein height levels on SLBs appears rather suited to conclude on ezrin's activation state.

The F-actin binding capability of ezrin was analyzed as ultimate measure for ezrin activation by force measurements (chapter 6). The influence of ezrin activation on the adhesion forces and surface adhesion energies between ezrin and F-actin was investigated by colloidal probe microscopy (CPM). An assay allowing the thorough characterization of the individual contributions of $\mathrm{PIP}_{2}$ and phosphorylation to the F-actin binding capability of ezrin was developed. F-actin was electrostatically attached to a self-assembled thiol monolayer (SAM), while the colloidal probe was functionalized with a SAM displaying NTA-Ni head groups, allowing the immobilization of ezrin via its $\mathrm{His}_{6}$-tag. Ezrin wildtype, ezrin $\mathrm{T}_{5} 67 \mathrm{~A}$, and ezrin $T_{567} \mathrm{D}$ were employed to assess the influence of phosphorylation on the interaction between ezrin and F-actin, while incubation of the respective protein with mono-dispersed $\mathrm{PIP}_{2}$ before its immobilization to the colloidal probe allowed to mimic $\mathrm{PIP}_{2}$ mediated activation. A significant increase in surface adhesion energy in presence of $\mathrm{PIP}_{2}$ was found, while phosphorylation contributed only to a minor extent on its own. However, both activation factors act synergistically to increase the surface adhesion energy between ezrin and F-actin. A relation between single molecule force measurements and CPM experiments was derived, accounting for the similar range of adhesion forces $<100 \mathrm{pN}$. Upon approximating the limiting case of a stiff transducer, single events could be detected in CPM experi- 
ments although an ensemble was probed. No distinct influence of ezrin activation on the adhesion forces between ezrin and F-actin was observable. Accordingly, it was shown for the first time that the strength of the interaction between ezrin and F-actin in vitro is rather defined by the increasing number of formed bonds than the enforcement of a single bond. The concept of combining many weak bonds to form strong intermolecular connections is employed within the cell to establish a stable yet dynamic interaction. 



\section{APPENDIX}

\section{A.I ABBREVIATIONS}

\begin{tabular}{|c|c|}
\hline AFM & Atomic force microscopy \\
\hline APS & Ammoniumperoxodisulfat \\
\hline $\mathrm{AUT}^{+}$-thiol & (11-mercaptoundecyl)trimethylammonium \\
\hline CD & Circular dichroism \\
\hline C-ERMAD & C-terminal ERM association domain \\
\hline CLSM & Confocal laser scanning microscopy \\
\hline DOGS-NTA-Ni & $\begin{array}{l}\text { 1,2-Dioleoyl-sn-glycero-3-[( } N \text {-(5-amino-1-carboxypentyl) } \\
\text { iminodiacetic acid)succinyl] (nickel salt) }\end{array}$ \\
\hline DPPC & 1,2-Dipalmitoyl-sn-glycero-3-phosphocholine \\
\hline DTS & Dodecyl-trichorosilane \\
\hline DTT & Dithiothreitol \\
\hline E. coli & Escherichia coli \\
\hline EDTA & Ethylene diamine tetraacetic acid \\
\hline EGTA & Ethylene glycol tetraacetic acid \\
\hline ERM & Ezrin, radixin, moesin \\
\hline F-actin & Filamentous actin \\
\hline FCS & Fluorescence correlation spectroscopy \\
\hline FD curve & Force distance curve \\
\hline FERM & Four-point-one-ERM \\
\hline FRAP & Fluorescence recovery after photobleaching \\
\hline G-actin & Globular actin \\
\hline GUV & Giant unilamellar vesicle \\
\hline $\mathrm{HCl}$ & Hydrochloric acid \\
\hline HEPES & 4-(2-Hydroxyethyl)-1-piperazineethanesulfonic acid \\
\hline $\mathrm{His}_{6}$ & $6 \times$ histidine \\
\hline InvOLS & Inverse optical lever sensitivity \\
\hline IPTG & Isopropyl $\beta$-D-1-thiogalactopyranoside \\
\hline
\end{tabular}


IQR

LB

LUV

MARCKS

Matrix thiol

MFP

MLV

MWCO

NA

NTA-Ni

N-ERMAD

NTA-thiol

N-WASP

OD

$\mathrm{PIP}_{2}$

POPC

rpm

QCM

RIfS

SAM

SDS-PAGE

SEM

SHM

SLB

SMFM

SSM

SPR

SUV

TEM

TEMED

TRIS

UV-VIS
Interquartile range

Lysogeny broth

Large unilamellar vesicle

Myristoylated alanine-rich $C$ kinase substrate

(1-mercaptoundec-11-yI)tri(ethylene glycol)

Molecular force probe

multilamellar vesicle

Molecular weight cut off

Numerical aperture

Nickel nitrilotriacetic acid

$\mathrm{N}$-terminal ERM association domain

$N$-[5-[[[(20-mercapto-3,6,9-trioxaeicos-1-yl)oxo]carbonyl]-

amino]-1-carboxypentyl]iminodiacetic acid

Neuronal WASP

Optical density

L- $\alpha$-Phosphatidylinositol-4,5-bisphosphate

1-Palmitoyl-2-oleoyl-sn-glycero-3-phosphocholine

Rounds per minute

Quartz crystal microbalance

Reflectometric interference spectroscopy

Self-assembled monolayer

Sodium dodecyl sulfate polyacrylamide gel electrophoresis

Scanning electron microscopy

Solid-supported hybrid membrane

Solid-supported lipid bilayer

Single molecule force measurement

Solid-supported membrane

Surface plasmon resonance

Small unilamellar vesicle

Transmission electron microscopy

Tetramethylethylenediamine

Tris(hydroxymethyl)aminomethane

Ultraviolet-visible 
WASP

wt

A.2 CHEMICALS
Wiskott-Aldrich syndrome protein

Wildtype
Acetic acid

Acrylamid-bisacrylamid

$\gamma$-actin

Agar

AlexaFluor488 phalloidin

AlexaFluor488 C5-maleimide

APS

Argon

$\mathrm{AUT}^{+}$-thiol

Bodipy- $\mathrm{C}_{12}-\mathrm{HPC}$

Bodipy TMR-PIP 2 (C16)

Bromphenol blue sodium salt

Chloroform

Chloramphenicol

Chromium

Complete mini EDTA-free

Coomassie Brilliant Blue G250

DOGS-NTA-Ni

DOPC

DTS

DTT

EDTA

EGTA

Ethanol p.a.

Glycerin

Glycin

Gold $9999 \%$
Carl Roth GmbH, Karlsruhe (Germany)

Carl Roth $\mathrm{GmbH}$, Karlsruhe (Germany)

Cytoskeleton, Denver (USA)

Carl Roth $\mathrm{GmbH}$, Karlsruhe (Germany)

Life Technologies, Darmstadt (Germany)

Life Technologies, Darmstadt (Germany)

Sigma Aldrich, Taufkirchen (Germany)

Air Liquide, Düsseldorf (Germany)

Prochimia, Sopot (Poland)

Life Technologies, Darmstadt (Germany)

Echelon Biosciences, Salt Lake City (USA)

Carl Roth $\mathrm{GmbH}$, Karlsruhe (Germany)

Merck, Darmstadt (Germany)

abcr GMbH, Karlsruhe (Germany)

Umicore Materials AG, Balzers (Liechtenstein)

Roche, Mannheim (Germany)

Carl Roth $\mathrm{GmbH}$, Karlsruhe

Avanti Polar Lipids, Alabaster (USA)

Avanti Polar Lipids, Alabaster (USA)

Sigma-Aldrich, Steinheim (Germany)

Sigma Aldrich, Taufkirchen (Germany)

Carl Roth GmbH, Karlsruhe (Germany)

Sigma Aldrich, Taufkirchen (Germany)

VWR International, Darmstadt (Germany)

Merck, Darmstadt (Germany)

Merck, Darmstadt (Germany)

Allgemeine Gold- und Silberscheideanstalt, Pforzheim (Germany) 
$\mathrm{HCl}$

HEPES

Hydrogen peroxide $30 \%$

Imidazole

IPTG

Kanamycin

Low molecular weight marker

Magnesium chloride

2-mercaptoethanol

Matrix thiol

Methanol, p.a.

Nickel chloride

NTA-Ni agarose

NTA-thiol

$\mathrm{PIP}_{2}$

POPC

Potassium chloride

SDS

Sodium azide

Sodium chloride

Sodium hydrogen carbonate

Sodium hydroxide

TEMED

Toluene p.a.

TRIS/HCl

Tryptone

Yeast extract
Sigma Aldrich, Taufkirchen (Germany)

Carl Roth GmbH, Karlsruhe (Germany)

VWR International, Darmstadt (Germany)

Sigma Aldrich, Taufkirchen (Germany)

Sigma Aldrich, Taufkirchen (Germany)

Sigma Aldrich, Taufkirchen (Germany)

GE Healthcare, Freiburg (Germany)

Merck, Darmstadt (Germany)

Carl Roth $\mathrm{GmbH}$, Karlsruhe (Germany)

Prochimia, Sopot (Poland)

VWR International, Darmstadt (Germany)

Merck, Darmstadt (Germany)

Qiagen, Hilden (Germany)

Prochimia, Sopot (Poland)

Avanti Polar Lipids, Alabaster (USA)

Avanti Polar Lipids, Alabaster (USA)

AppliChem, Darmstadt (Germany)

Merck, Darmstadt (Germany)

Merck, Darmstadt (Germany)

VWR International, Darmstadt (Germany)

Carl Roth GmbH, Karlsruhe (Germany)

Merck, Darmstadt (Germany)

Sigma Aldrich, Taufkirchen (Germany)

VWR, Darmstadt (Germany)

Carl Roth $\mathrm{GmbH}$, Karlsruhe (Germany)

Carl Roth $\mathrm{GmbH}$, Karlsruhe (Germany)

Carl Roth GmbH, Karlsruhe (Germany)

\section{A.3 HARDWARE}

Allegra $^{\mathrm{TM}} \mathrm{X}-22 \mathrm{R}$, Rotor $\mathrm{SX}_{4250}$

Cary 50 UV spectrometer
Beckman Coulter, Krefeld (Germany)

Varian, Darmstadt (Germany) 
Galaxy mini

Gel electrophoresis system PerfectBlue Twin

Heraeus Fresco 17

Impedance/Gain-Phase Analyzer SI 1260

JPK Nanowizard III

LEO SUPRA 35 SEM microscope

LSM 710

MED o20 Modular High Vacuum Coating System

MFP-3D
Milli-Q plus 185
Milli-RO 3
NanoDrop spectrophotometer
Optima $^{\mathrm{TM}}$ L-9oK, Rotor Ti70
Peristaltic pump
pH-Meter Calimatic 766
Plasma cleaner
Res-Tec2005 spectrometer

Rotator

Shaking incubator $\mathrm{SM}_{30}$

Sonopuls HD 2070, resonator cup

Thermomixer compact

Transilluminator

Ultrasonic bath Sonorex

Vacuum oven

Vortex mixer Reax Top
VWR International, Darmstadt (Germany)

PEQLAB Biotechnologie, Erlangen (Germany)

Thermo, Waltham (USA) (Germany)

Solartron Instruments, Farnborough (UK)

JPK Instruments, Berlin (Germany)

Zeiss, Jena (Germany)

Zeiss, Jena (Germany)

Bal-Tec AG, Balzers (Liechtenstein)

Asylum Research, Santa Barbara (USA)

Millipore, Eschborn (Germany)

Millipore, Eschborn (Germany)

Thermo Scientific, Wilmington (USA)

Beckman Coulter, Krefeld (Germany)

Ismatec, Wertheim (Germany)

Knick, Berlin (Germany)

Harrick, New York City (USA)

Resonant Technologies, Framersheim (Germany)

VWR International, Darmstadt (Germany)

Edmund Bühler GmbH, Tübingen (Germany)

Bandelin, Berlin (Germany)

Eppendorf, Hamburg (Germany)

Vilber Lourmat, Eberhardzell (Germany)

Bandelin, Berlin (Germany)

Binder, Tuttlingen (Germany)

Heidolph, Schwabach (Germany) 
Biolever

Colloidal probes

CSC 37 cantilevers

Dialysis Tubing

LaSFN9 glasses

Molecular sieves $4 \AA$

Peristaltic pump tubing

RIfS transducer chips

Silicon wafers

Vivaspin $500 \& 6$
Olympus, Tokyo (Japan)

sQube, Bickenbach (Germany)

MikroMasch, Wetzlar (Germany)

Carl Roth, Karlsruhe (Germany)

Hellma Optik, Halle (Germany)

Carl Roth, Karlsruhe (Germany)

Ismatec, Wertheim (Germany)

ABC - Active business company,

München (Germany)

Silicon materials, Kaufering (Germany)

Sartorius, Göttingen (Germany)

\title{
A. 5 SYMBOLS
}

$A_{\mathrm{H}}$
$a_{\mathrm{Hertz}}$
$A_{\mathrm{Hertz}}$
$c$
$C$
$C_{\mathrm{D}}$
$C_{\mathrm{K}}$
$C_{\mathrm{L}}$
$\delta$
$d$
$D$
$E$
$E$
E

\author{
Absorbance \\ Hamaker constant \\ Contact radius according to Hertz model \\ Contact area according to Hertz model \\ Concentration, Speed of light \\ Coefficient in the surface-surface poten- \\ tial \\ Contribution of Debye force \\ Contribution of Keesom force \\ Contribution of London force \\ Indentation \\ Physical layer thickness \\ Diffusion coefficient, Distance \\ Young's modulus \\ Molar extinction coefficient, Dielectric \\ constant \\ Applied force per bond
}




\begin{tabular}{|c|c|}
\hline$F$ & Force \\
\hline$F_{\mathrm{ad}}$ & Adhesion force \\
\hline$I$ & Intensity \\
\hline$k$ & Wave vector \\
\hline$k_{\mathrm{B}}$ & Boltzmann's constant \\
\hline$k_{c}$ & Cantilever stiffness/ spring constant \\
\hline$k_{\mathrm{off}}^{0}$ & Unbinding rate at zero force \\
\hline$K_{\mathrm{d}}$ & Dissociation constant/ binding affinity \\
\hline$l$ & Pathlength \\
\hline$L$ & Length \\
\hline$\lambda$ & Wavelength \\
\hline$n$ & Refractive index \\
\hline OT & Optical thickness \\
\hline$\rho$ & Number of atoms per unit volume \\
\hline$R$ & Radius, Reflectivity \\
\hline$r$ & Fresnel coefficient \\
\hline$T$ & Temperature \\
\hline$\Theta$ & Angle of incidence \\
\hline$t_{\mathrm{c}}$ & Cantilever thickness \\
\hline$\tau_{\mathrm{D}}$ & Diffusion time \\
\hline$\theta$ & Cantilever tilt \\
\hline$v_{F}$ & Loading rate \\
\hline$V$ & Interaction potential \\
\hline$\omega$ & Circular frequency \\
\hline$W_{\mathrm{ad}}$ & Surface adhesion energy \\
\hline$w$ & $\begin{array}{l}\text { Overall VdW-contribution to interaction } \\
\text { potential, Bin width }\end{array}$ \\
\hline$w_{\mathrm{c}}$ & Cantilever width \\
\hline$w_{\mathrm{G}}$ & Gauss radius \\
\hline$x^{\ddagger}$ & Potential width \\
\hline$Z_{\mathrm{c}}$ & Cantilever deflection \\
\hline$Z_{\mathrm{p}}$ & z-Piezo displacement \\
\hline
\end{tabular}




\section{MATLAB CODE}

In the following an example of a MATLAB script is given on how histogram, kernel density, and empirical cumulative distribution functions were determined.

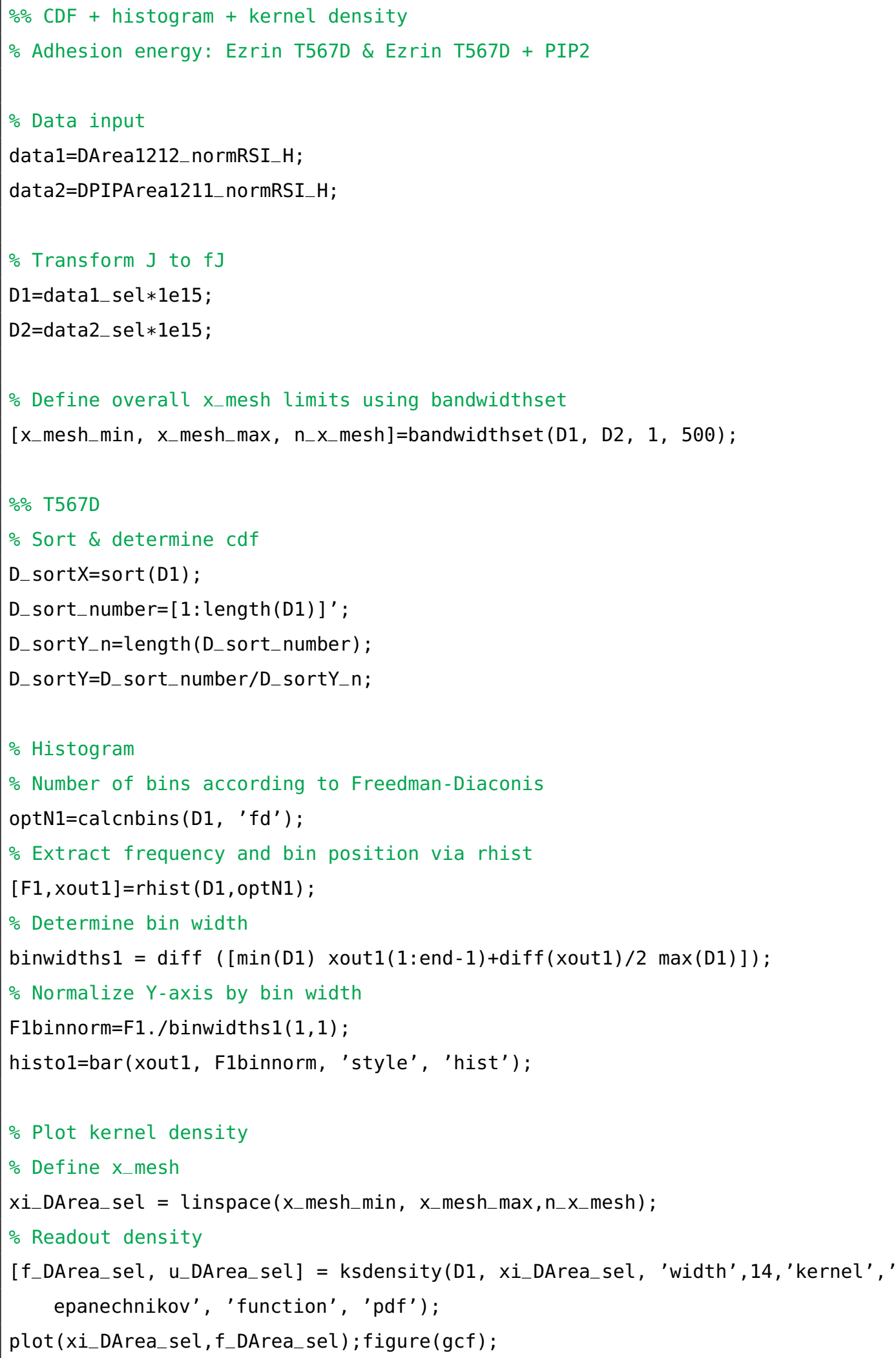




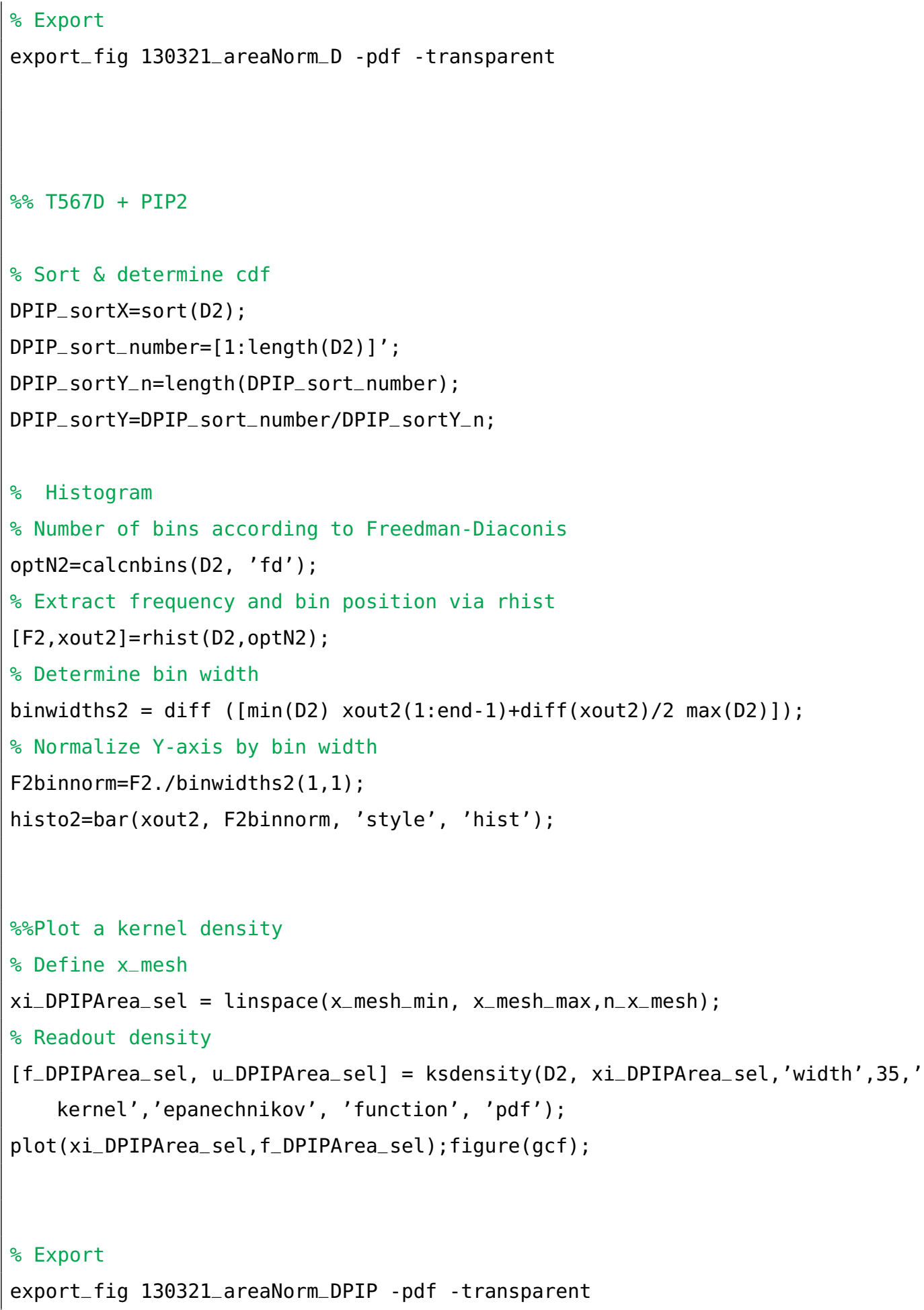



[1] Fehon, R. G, McClatchey, A. I, \& Bretscher, A. (2010) Organizing the cell cortex: the role of ERM proteins. Nature Reviews Molecular Cell Biology 11, $276-287$.

[2] Doi, Y, Itoh, M, Yonemura, S, Ishihara, S, Takano, H, Noda, T, Tsukita, S, \& Tsukita, S. (1999) Normal development of mice and unimpaired cell adhesion/cell motility/actin-based cytoskeleton without compensatory upregulation of ezrin or radixin in moesin gene knockout. Journal of Biological Chemistry 274, 2315-2321.

[3] Bretscher, A, Edwards, K, \& Fehon, R. G. (2002) ERM proteins and merlin: integrators at the cell cortex. Nature Reviews Molecular Cell Biology 3, 586-599.

[4] Arpin, M, Chirivino, D, Naba, A, \& Zwaenepoel, I. (2011) Emerging role for ERM proteins in cell adhesion and migration. Cell Adhesion $\mathcal{E}$ Migration 5, 199-206.

[5] Casaletto, J. B, Saotome, I, Curto, M, \& McClatchey, A. I. (2011) Ezrinmediated apical integrity is required for intestinal homeostasis. Proceedings of the National Academy of Sciences 108, 11924-11929.

[6] Turunen, O, Wahlström, T, \& Vaheri, A. (1994) Ezrin has a COOH-terminal actin-binding site that is conserved in the ezrin protein family. Journal of Cell Biology 126, 1445-1453.

[7] Gary, R \& Bretscher, A. (1995) Ezrin self-association involves binding of an N-terminal domain to a normally masked C-terminal domain that includes the F-actin binding site. Molecular Biology of the Cell 6, 1061.

[8] Nakamura, F, Amieva, M. R, \& Furthmayr, H. (1995) Phosphorylation of threonine 558 in the carboxyl-terminal actin-binding domain of moesin by thrombin activation of human platelets. Journal of Biological Chemistry 270, $31377-31385$.

[9] Matsui, T, Maeda, M, Doi, Y, Yonemura, S, Amano, M, Kaibuchi, K, Tsukita, S, \& Tsukita, S. (1998) Rho-kinase phosphorylates COOH-terminal threonines of ezrin/radixin/moesin (ERM) proteins and regulates their head-to-tail association. Journal of Cell Biology 140, 647-657. 
[10] Niggli, V, Andréoli, C, Roy, C, \& Mangeat, P. (1995) Identification of a phosphatidylinositol-4,5-bisphosphate-binding domain in the n-terminal region of ezrin. FEBS Letters 376, 172-176.

[11] Yonemura, S, Matsui, T, Tsukita, S, \& Tsukita, S. (2002) Rho-dependent andindependent activation mechanisms of ezrin/radixin/moesin proteins: an essential role for polyphosphoinositides in vivo. Journal of Cell Science 115, 2569-2580.

[12] Fievet, B. T, Gautreau, A, Roy, C, Del Maestro, L, Mangeat, P, Louvard, D, \& Arpin, M. (2004) Phosphoinositide binding and phosphorylation act sequentially in the activation mechanism of ezrin. Journal of Cell Biology 164, 653-659.

[13] Roch, F, Polesello, C, Roubinet, C, Martin, M, Roy, C, Valenti, P, Carreno, S, Mangeat, P, \& Payre, F. (2010) Differential roles of PtdIns $(4,5) \mathrm{P} 2$ and phosphorylation in moesin activation during Drosophila development. Journal of Cell Science 123, 2058-2067.

[14] Bosk, S, Braunger, J. A, Gerke, V, \& Steinem, C. (2011) Activation of F-actin binding capacity of ezrin: Synergism of PIP2 interaction and phosphorylation. Biophysical Journal 100, 1708-1717.

[15] Janke, M, Herrig, A, Austermann, J, Gerke, V, Steinem, C, \& Janshoff, A. (2008) Actin binding of ezrin is activated by specific recognition of PIP2functionalized lipid bilayers. Biochemistry 47, 3762-3769.

[16] Studier, F \& Moffatt, B. (1986) Use of bacteriophage $T_{7}$ RNA polymerase to direct selective high-level expression of cloned genes. Journal of Molecular Biology 189, 113.

[17] Studier, F et al. (1991) Use of bacteriophage $\mathrm{T}_{7}$ lysozyme to improve an inducible $\mathrm{T}_{7}$ expression system. Journal of Molecular Biology 219, 37.

[18] Baneyx, F et al. (1999) Recombinant protein expression in Escherichia coli. Current Opinion in Biotechnology 10, 411-421.

[19] Laemmli, U. (1970) Cleavage of structural proteins during the assembly of the head of bacteriophage $T_{4}$. Nature 227, 680-685.

[20] Eftink, M. R. (2006) Fluorescence Techniques for Studying Protein Structure. (John Wiley \& Sons, Inc.).

[21] Gasteiger, E, Hoogland, C, Gattiker, A, Duvaud, S, Wilkins, M. R, Appel, R. D, \& Bairoch, A. (2005) The Proteomics Protocols Handbook ed. Walker, J. M. (Springer-Verlag). 
[22] Nuzzo, R. G \& Allara, D. L. (1983) Adsorption of bifunctional organic disulfides on gold surfaces. Journal of the American Chemical Society 105, 4481-4483.

[23] Maoz, R \& Sagiv, J. (1984) On the formation and structure of self-assembling monolayers. I. A comparative ATR-wettability study of Langmuir-Blodgett and adsorbed films on flat substrates and glass microbeads. Journal of Colloid and Interface Science 100, 465-496.

[24] Brian, A. A \& McConnell, H. M. (1984) Allogeneic stimulation of cytotoxic T cells by supported planar membranes. Proceedings of the National Academy of Sciences 81, 6159-6163.

[25] Seul, M, Subramaniam, S, \& McConnell, H. M. (1985) Mono- and bilayers of phospholipids at interfaces: interlayer coupling and phase stability. Journal of Physical Chemistry 89, 3592-3595.

[26] Plant, A. L. (1993) Self-assembled phospholipid/alkanethiol biomimetic bilayers on gold. Langmuir 9, 2764-2767.

[27] Spinke, J, Liley, M, Schmitt, F.-J, Guder, H.-J, Angermaier, L, \& Knoll, W. (1993) Molecular recognition at self-assembled monolayers: Optimization of surface functionalization. Journal of Chemical Physics 99, 7012.

[28] Sigal, G. B, Bamdad, C, Barberis, A, Strominger, J, \& Whitesides, G. M. (1996) A self-assembled monolayer for the binding and study of histidine-tagged proteins by surface plasmon resonance. Analytical Chemistry 68, 490-497.

[29] Lazzara, T. D, Kliesch, T.-T, Janshoff, A, \& Steinem, C. (2011) Orthogonal functionalization of nanoporous substrates: Control of $3 \mathrm{D}$ surface functionality. ACS Applied Materials \& Interfaces 3, 1068-1076.

[30] Tamm, L \& McConnell, H. (1985) Supported phospholipid bilayers. Biophysical Journal 47, $105-113$.

[31] Cremer, P. S \& Boxer, S. G. (1999) Formation and spreading of lipid bilayers on planar glass supports. Journal of Physical Chemistry B 103, 2554-2559.

[32] Zasadzinski, J, Helm, C, Longo, M, Weisenhorn, A, Gould, S, \& Hansma, P. (1991) Atomic force microscopy of hydrated phosphatidylethanolamine bilayers. Biophysical Journal 59, $755-760$.

[33] Binning, G, Quate, C, \& Gerber, C. (1986) Atomic force microscope. Physical Review Letters 56, 930-933.

[34] Butt, H, Downing, K, \& Hansma, P. (1990) Imaging the membrane protein bacteriorhodopsin with the atomic force microscope. Biophysical Journal 58, 1473 . 
[35] Butt, H, Wolff, E, Gould, S, Dixon Northern, B, Peterson, C, \& Hansma, P. (1990) Imaging cells with the atomic force microscope. Journal of Structural Biology 105, 54-61.

[36] Jandt, K, Heier, J, Bates, F, \& Kramer, E. (1996) Transient surface roughening of thin films of phase separating polymer mixtures. Langmuir 12, 3716-3720.

[37] Orisaka, S, Minobe, T, Uchihashi, T, Sugawara, Y, \& Morita, S. (1999) The atomic resolution imaging of metallic Ag (111) surface by noncontact atomic force microscope. Applied Surface Science 140, 243-246.

[38] Martin, Y, Williams, C, \& Wickramasinghe, H. (1987) Atomic force microscope-force mapping and profiling on a sub 100- $\AA$ scale. Journal of Applied Physics 61, 4723-4729.

[39] Florin, E.-L, Moy, V. T, \& Gaub, H. E. (1994) Adhesion forces between individual ligand-receptor pairs. Science 264, 415-417.

[40] Rief, M, Gautel, M, Oesterhelt, F, Fernandez, J, \& Gaub, H. (1997) Reversible unfolding of individual titin immunoglobulin domains by afm. Science 276, 1109-1112.

[41] Gould, S, Drake, B, Prater, C, Weisenhorn, A, Manne, S, Kelderman, G, Butt, H.-J, Hansma, H, Hansma, P, Magonov, S, et al. (1990) The atomic force microscope: a tool for science and industry. Ultramicroscopy 33, 93-98.

[42] Tompkins, B, Manos, C, \& Salatino, A. (2008) MFP-3D Installation and Operation Manual (Asylum Research).

[43] Jandt, K. (2001) Atomic force microscopy of biomaterials surfaces and interfaces. Surface Science 491, 303-332.

[44] Butt, H.-J, Cappella, B, \& Kappl, M. (2005) Force measurements with the atomic force microscope: Technique, interpretation and applications. Surface Science Reports 59, 1-152.

[45] Hamaker, H. (1937) The London-van der Waals attraction between spherical particles. Physica 4, 1058-1072.

[46] Israelachvili, J. N. (1992) Intermolecular and Surface Forces, Second Edition: With Applications to Colloidal and Biological Systems (Colloid Science). (Academic Press).

[47] Sarid, D. (1991) Review of scanning force microscopy. Journal of Vacuum Science \& Technology B: Microelectronics and Nanometer Structures 9, 431. 
[48] Drummond, C. J \& Senden, T. J. (1995) Interfaces II (Materials Science Forum) ed. Muddle, B. C. (Trans Tech Pubn), pp. 107-113.

[49] Hutter, J. L \& Bechhoefer, J. (1993) Calibration of atomic-force microscope tips. Review of Scientific Instruments 64, 1868-1873.

[50] Torii, A, Sasaki, M, Hane, K, \& Okuma, S. (1996) A method for determining the spring constant of cantilevers for atomic force microscopy. Measurement Science and Technology 7, 179.

[51] Cleveland, J. P, Manne, S, Bocek, D, \& Hansma, P. K. (1993) A nondestructive method for determining the spring constant of cantilevers for scanning force microscopy. Review of Scientific Instruments 64, 403-405.

[52] Butt, H. J \& Jaschke, M. (1995) Calculation of thermal noise in atomic force microscopy. Nanotechnology 6, 1-7.

[53] Walters, D. A, Cleveland, J. P, Thomson, N. H, Hansma, P. K, Wendman, M. A, Gurley, G, \& Elings, V. (1996) Short cantilevers for atomic force microscopy. Review of Scientific Instruments 67, 3583 .

[54] Proksch, R, Schäffer, T. E, Cleveland, J. P, Callahan, R. C, \& Viani, M. B. (2004) Finite optical spot size and position corrections in thermal spring constant calibration. Nanotechnology 15, 1344-1350.

[55] Heim, L.-O, Kappl, M, \& Butt, H.-J. (2004) Tilt of atomic force microscope cantilevers: Effect on spring constant and adhesion measurements. Langmuir 20, $2760-2764$.

[56] Hutter, J. L. (2005) Comment on tilt of atomic force microscope cantilevers: Effect on spring constant and adhesion measurements. Langmuir 21, 26302632.

[57] Durkan, C. (2007) Current at the Nanoscale: An Introduction to Nanoelectronics. (Imperial College Press).

[58] García, R. (2002) Dynamic atomic force microscopy methods. Surface Science Reports 47, 197-301.

[59] Dürrenberger, M. (2001) Confocal laser scanning microscopy within the field of biomaterials. European Cells and Materials 1, 10-11.

[6o] Brakenhoff, G, Van der Voort, H, Van Spronsen, E, Nanninga, N, et al. (1988) 3-dimensional imaging of biological structures by high resolution confocal scanning laser microscopy. Scanning Microscopy 2, 33. 
[61] Singer, S. J \& Nicolson, G. L. (1972) The fluid mosaic model of the structure of cell membranes. Science 175, 720-731.

[62] Peters, R, Peters, J, Tews, K, \& Bähr, W. (1974) A microfluorimetric study of translational diffusion in erythrocyte membranes. Biochimica et Biophysica Acta, Biomembranes 367, 282-294.

[63] Axelrod, D, Koppel, D, Schlessinger, J, Elson, E, \& Webb, W. (1976) Mobility measurement by analysis of fluorescence photobleaching recovery kinetics. Biophysical Journal 16, 1055.

[64] Seiffert, S \& Oppermann, W. (2005) Systematic evaluation of FRAP experiments performed in a confocal laser scanning microscope. Journal of $\mathrm{Mi}$ croscopy 220, 20-30.

[65] Jönsson, P, Jonsson, M. P, Tegenfeldt, J. O, \& Höök, F. (2008) A method improving the accuracy of fluorescence recovery after photobleaching analysis. Biophysical Journal 95, $5334-5348$.

[66] Jönnson, P. (2008) Frap analysis program. http://www.mpjonsson.com/frap3.html.

[67] Gauglitz, G. (2006) Optical Chemical Sensors, 217-237 eds. Baldini, F, Chester, A. N, Homola, J, \& Martellucci, S. (Springer Berlin / Heidelberg) Vol. 224.

[68] Gauglitz, G. (2010) Direct optical detection in bioanalysis: an update. Analytical and Bioanalytical Chemistry 398, 2363-2372.

[69] Halliday, D, Resnick, R, \& Walker, J. (2010) Fundamentals of Physics. (John Wiley \& Sons).

[70] Naumann, R, Schmidt, E, Jonczyk, A, Fendler, K, Kadenbach, B, Liebermann, T, Offenhäusser, A, \& Knoll, W. (1999) The peptide-tethered lipid membrane as a biomimetic system to incorporate cytochrome c oxidase in a functionally active form. Biosensors and Bioelectronics 14, 651-662.

[71] Benesch, J, Askendal, A, \& Tengvall, P. (2002) The determination of thickness and surface mass density of mesothick immunoprecipitate layers by null ellipsometry and protein ${ }^{125}$ Iodine labeling. Journal of Colloid and Interface Science 249, $84-90$.

[72] Vörös, J. (2004) The density and refractive index of adsorbing protein layers. Biophysical Journal 87, $553-561$.

[73] Schmitt, H.-M, Brecht, A, Piehler, J, \& Gauglitz, G. (1997) An integrated system for optical biomolecular interaction analysis. Biosensors and Bioelectronics 12, 809-816. 
[74] Ghosh, G. (1999) Dispersion-equation coefficients for the refractive index and birefringence of calcite and quartz crystals. Optics Communications 163, 95-102.

[75] Boardman, A. (1982) Electromagnetic Surface Modes. (John Wiley \& Sons).

[76] Sambles, J. R, Bradbery, G. W, \& Yang, F. (1991) Optical excitation of surface plasmons: An introduction. Contemporary Physics 32, 173-183.

[77] Liedberg, B, Lundström, I, \& Stenberg, E. (1993) Principles of biosensing with an extended coupling matrix and surface plasmon resonance. Sensors and Actuators B: Chemical 11, $63-72$.

[78] Homola, J. (2008) Surface plasmon resonance sensors for detection of chemical and biological species. Chemical Reviews 108, 462-493.

[79] Dunlap, M \& Adaskaveg, J. E. (1997) Introduction to the Scanning Electron Microscope: Theory, Practice, \& Procedures (FACILITY FOR ADVANCED INSTRUMENTATION, U. C. Davis).

[8o] Raucher, D, Stauffer, T, Chen, W, Shen, K, Guo, S, York, J. D, Sheetz, M. P, \& Meyer, T. (2000) Phosphatidylinositol 4, 5-bisphosphate functions as a second messenger that regulates cytoskeleton-plasma membrane adhesion. Cell 1oo, $221-228$.

[81] Liscovitch, M, Chalifa, V, Pertile, P, Chen, C.-S, \& Cantley, L. C. (1994) Novel function of phosphatidylinositol 4,5-bisphosphate as a cofactor for brain membrane phospholipase D. Journal of Biological Chemistry 269, 2140321406.

[82] Hansen, S. B, Tao, X, \& MacKinnon, R. (2011) Structural basis of PIP2 activation of the classical inward rectifier $\mathrm{K}^{+}$channel Kir2.2. Nature 477, 495-498.

[83] Cremona, O, Di Paolo, G, Wenk, M. R, Lüthi, A, Kim, W. T, Takei, K, Daniell, L, Nemoto, Y, Shears, S. B, Flavell, R. A, et al. (1999) Essential role of phosphoinositide metabolism in synaptic vesicle recycling. Cell 99, 179-188.

[84] Simonsen, A, Wurmser, A. E, Emr, S. D, \& Stenmark, H. (2001) The role of phosphoinositides in membrane transport. Current Opinion in Cell Biology 13, 485-492.

[85] Martin, T. F. (2001) PI $(4,5) \mathrm{P} 2$ regulation of surface membrane traffic. Current Opinion in Cell Biology 13, 493-499.

[86] Li Zhang, Yuntao S. Mao, P. A. J \& Yin, H. L. (2012) Phosphoinositides II: The Diverse Biological Functions eds. Balla, T, Wymann, M, \& York, J. D. (Springer Netherlands). 
[87] Lupyan, D, Mezei, M, Logothetis, D. E, \& Osman, R. (2010) A molecular dynamics investigation of lipid bilayer perturbation by PIP2. Biophysical Journal 98, 240-247.

[88] McLaughlin, S, Wang, J, Gambhir, A, \& Murray, D. (2002) PIP2 and proteins: interactions, organization, and information flow. Annual Review of Biophysics and Biomolecular Structure 31, 151-175.

[89] Miki, H, Miura, K, \& Takenawa, T. (1996) N-WASP, a novel actindepolymerizing protein, regulates the cortical cytoskeletal rearrangement in a PIP2-dependent manner downstream of tyrosine kinases. EMBO Journal 15, 5326.

[9o] Rohatgi, R, Ma, L, Miki, H, Lopez, M, Kirchhausen, T, Takenawa, T, \& Kirschner, M. W. (1999) The interaction between N-WASP and the Arp2/3 complex links Cdc42-dependent signals to actin assembly. Cell 97, 221-231.

[91] Yonezawa, N, Nishida, E, Iida, K, Yahara, I, \& Sakai, H. (1990) Inhibition of the interactions of cofilin, destrin, and deoxyribonuclease I with actin by phosphoinositides. Journal of Biological Chemistry 265, 8382-8386.

[92] Lappalainen, P \& Drubin, D. G. (1997) Cofilin promotes rapid actin filament turnover in vivo. Nature $388,78-82$.

[93] Palmgren, S, Ojala, P. J, Wear, M. A, Cooper, J. A, \& Lappalainen, P. (2001) Interactions with $\mathrm{PIP} 2, \mathrm{ADP}$-actin monomers, and capping protein regulate the activity and localization of yeast twinfilin. Journal of Cell Biology 155, 251260 .

[94] McLaughlin, S \& Murray, D. (2005) Plasma membrane phosphoinositide organization by protein electrostatics. Nature 438, 605-611.

[95] Balla, T, Bondeva, T, \& Várnai, P. (2000) How accurately can we image inositol lipids in living cells? Trends in Pharmacological Sciences 21, 238-241.

[96] Janmey, P. A \& Lindberg, U. (2004) Cytoskeletal regulation: rich in lipids. Nature Reviews Molecular Cell Biology 5, 658-666.

[97] Gambhir, A, Hangyás-Mihályné, G, Zaitseva, I, Cafiso, D. S, Wang, J, Murray, D, Pentyala, S. N, Smith, S. O, \& McLaughlin, S. (2004) Electrostatic sequestration of $\mathrm{PIP} 2$ on phospholipid membranes by basic/aromatic regions of proteins. Biophysical Journal 86, $2188-2207$.

[98] Glaser, M, Wanaski, S, Buser, C. A, Boguslavsky, V, Rashidzada, W, Morris, A, Rebecchi, M, Scarlata, S. F, Runnels, L. W, Prestwich, G. D, et al. 
(1996) Myristoylated alanine-rich C kinase substrate (MARCKS) produces reversible inhibition of phospholipase $C$ by sequestering phosphatidylinositol 4,5-bisphosphate in lateral domains. Journal of Biological Chemistry 271, 26187-26193.

[99] Wang, J, Arbuzova, A, Hangyás-Mihályné, G, \& McLaughlin, S. (2001) The effector domain of myristoylated alanine-rich $C$ kinase substrate binds strongly to phosphatidylinositol 4,5-bisphosphate. Journal of Biological Chemistry 276, 5012-5019.

[10o] Ohmori, S, Sakai, N, Shirai, Y, Yamamoto, H, Miyamoto, E, Shimizu, N, \& Saito, N. (2000) Importance of protein kinase C targeting for the phosphorylation of its substrate, myristoylated alanine-rich C-kinase substrate. Journal of Biological Chemistry 275, 26449-26457.

[101] Carvalho, K, Ramos, L, Roy, C, \& Picart, C. (2008) Giant unilamellar vesicles containing phosphatidylinositol $(4,5)$ bisphosphate: Characterization and functionality. Biophysical Journal 95, 4348-4360.

[102] Levental, I, Christian, D. A, Wang, Y.-H, Madara, J. J, Discher, D. E, \& Janmey, P. A. (2009) Calcium-dependent lateral organization in phosphatidylinositol 4,5-bisphosphate ( $\left.\mathrm{PIP}_{2}\right)$ - and cholesterol-containing monolayers. Biochemistry 48, 8241-8248. PMID: 19630438.

[103] Ellenbroek, W, Wang, Y.-H, Christian, D, Discher, D, Janmey, P, \& Liu, A. (2011) Divalent cation-dependent formation of electrostatic PIP2 clusters in lipid monolayers. Biophysical Journal 101, $2178-2184$.

[104] Wilson, L, Matsudaira, P. T, \& Nuccitelli, R. (1994) A practical guide to the study of calcium in living cells. (Academic Press) Vol. 4o.

[105] Castellana, E. T \& Cremer, P. S. (2006) Solid supported lipid bilayers: From biophysical studies to sensor design. Surface Science Reports 61, 429-444.

[106] Wilschut, J \& Hoekstra, D. (1986) Membrane fusion: lipid vesicles as a model system. Chemistry and Physics of Lipids 40, 145-166.

[107] Ong, S, Zhao, X, \& Eisenthal, K. B. (1992) Polarization of water molecules at a charged interface: second harmonic studies of the silica/water interface. Chemical Physics Letters 191, 327-335.

[108] van Paridon, P. A, de Kruijff, B, Ouwerkerk, R, \& Wirtz, K. W. (1986) Polyphosphoinositides undergo charge neutralization in the physiological pH range: a ${ }^{31} \mathrm{P}-\mathrm{NMR}$ study. Biochimica et Biophysica Acta, Lipids and Lipid Metabolism 877, 216-219. 
[109] Richter, R. P, Bérat, R, \& Brisson, A. R. (2006) Formation of solid-supported lipid bilayers: an integrated view. Langmuir 22, 3497-3505.

[110] Rädler, J, Strey, H, \& Sackmann, E. (1995) Phenomenology and kinetics of lipid bilayer spreading on hydrophilic surfaces. Langmuir 11, 4539-4548.

[111] Eisenberg, M, Gresalfi, T, Riccio, T, \& McLaughlin, S. (1979) Adsorption of monovalent cations to bilayer membranes containing negative phospholipids. Biochemistry 18, 5213-5223.

[112] Csúcs, G \& Ramsden, J. J. (1998) Interaction of phospholipid vesicles with smooth metal-oxide surfaces. Biochimica et Biophysica Acta, Biomembranes 1369, 61-70.

[113] Ohki, S \& Ohshima, H. (1999) Interaction and aggregation of lipid vesicles (DLVO theory versus modified DLVO theory). Colloids and Surfaces B: Biointerfaces 14, 27-45.

[114] Richter, R, Mukhopadhyay, A, \& Brisson, A. (2003) Pathways of lipid vesicle deposition on solid surfaces: a combined QCM-D and AFM study. Biophysical Journal 85, 3035-3047.

[115] Fernandes, F, Loura, L. M. S, Fedorov, A, \& Prieto, M. (2006) Absence of clustering of phosphatidylinositol-(4,5)-bisphosphate in fluid phosphatidylcholine. Journal of Lipid Research 47, 1521-1525.

[116] Toker, A. (1998) The synthesis and cellular roles of phosphatidylinositol 4,5bisphosphate. Current Opinion in Cell Biology 10, 254-261.

[117] Herrig, A, Janke, M, Austermann, J, Gerke, V, Janshoff, A, \& Steinem, C. (2006) Cooperative adsorption of ezrin on PIP2-containing membranes. Biochemistry 45, 13025-13034.

[118] Wang, J. (2002) Lateral sequestration of phosphatidylinositol 4,5bisphosphate by the basic effector domain of myristoylated alanine-rich $\mathrm{c}$ kinase substrate is due to nonspecific electrostatic interactions. Journal of Biological Chemistry 277, 34401-34412.

[119] Blin, G, Margeat, E, Carvalho, K, Royer, C. A, Roy, C, \& Picart, C. (2008) Quantitative analysis of the binding of ezrin to large unilamellar vesicles containing phosphatidylinositol 4,5 bisphosphate. Biophysical journal 94, 10211033.

[120] Ben-Aissa, K, Patino-Lopez, G, Belkina, N. V, Maniti, O, Rosales, T, Hao, J.J, Kruhlak, M. J, Knutson, J. R, Picart, C, \& Shaw, S. (2012) Activation of 
moesin, a protein that links actin cytoskeleton to the plasma membrane, occurs by phosphatidylinositol 4,5-bisphosphate (PIP2) binding sequentially to two sites and releasing an autoinhibitory linker. Journal of Biological Chemistry 287, 16311-16323.

[121] Liu, A. P \& Fletcher, D. A. (2006) Actin polymerization serves as a membrane domain switch in model lipid bilayers. Biophysical Journal 91, 4064-4070.

[122] Janshoff, A \& Steinem, C. (2006) Transport across artificial membranes-an analytical perspective. Analytical and Bioanalytical Chemistry 385, 433-451.

[123] Bosk, S. (2011) Ph.D. thesis (Georg-August University Göttingen).

[124] Niggli, V. (2001) Structural properties of lipid-binding sites in cytoskeletal proteins. Trends in Biochemical Sciences 26, 604-611.

[125] Tawa, K \& Morigaki, K. (2005) Substrate-supported phospholipid membranes studied by surface plasmon resonance and surface plasmon fluorescence spectroscopy. Biophysical Journal 89, 2750-2758.

[126] Kučerka, N, Nieh, M.-P, \& Katsaras, J. (2011) Fluid phase lipid areas and bilayer thicknesses of commonly used phosphatidylcholines as a function of temperature. Biochimica et Biophysica Acta, Biomembranes 1808, 2761-2771.

[127] Boussaad, S, Pean, J, \& Tao, N. J. (2000) High-resolution multiwavelength surface plasmon resonance spectroscopy for probing conformational and electronic changes in redox proteins. Analytical Chemistry 72, 222-226.

[128] Wang, Y.-H, Collins, A, Guo, L, Smith-Dupont, K. B, Gai, F, Svitkina, T, \& Janmey, P. A. (2012) Divalent cation-induced cluster formation by polyphosphoinositides in model membranes. Journal of the American Chemical Society 134, 3387-3395.

[129] Levental, I, Janmey, P, \& Cēbers, A. (2008) Electrostatic contribution to the surface pressure of charged monolayers containing polyphosphoinositides. Biophysical Journal 95, 1199-1205.

[130] Portzehl, H, Caldwell, P, \& Rüegg, J. (1964) The dependence of contraction and relaxation of muscle fibres from the crab maia squinado on the internal concentration of free calcium ions. Biochimica et Biophysica Acta 79, 581 .

[131] Liepiņa, I, Czaplewski, C, Janmey, P, \& Liwo, A. (2003) Molecular dynamics study of a gelsolin-derived peptide binding to a lipid bilayer containing phosphatidylinositol 4,5-bisphosphate. Peptide Science 71, 49-70. 
[132] Redfern, D. A \& Gericke, A. (2005) ph-dependent domain formation in phosphatidylinositol polyphosphate/phosphatidylcholine mixed vesicles. Journal of Lipid Research 46, 504-515.

[133] Levental, I, Cēbers, A, \& Janmey, P. A. (2008) Combined electrostatics and hydrogen bonding determine intermolecular interactions between polyphosphoinositides. Journal of the American Chemical Society 130, 9025-9030.

[134] Rossetti, F. F, Textor, M, \& Reviakine, I. (2006) Asymmetric distribution of phosphatidyl serine in supported phospholipid bilayers on titanium dioxide. Langmuir 22, 3467-3473.

[135] Köchy, T \& Bayerl, T. M. (1993) Lateral diffusion coefficients of phospholipids in spherical bilayers on a solid support measured by ${ }^{2} \mathrm{H}$-nuclear-magneticresonance relaxation. Physical Review E 47, 2109.

[136] Johnson, S, Bayerl, T, McDermott, D, Adam, G, Rennie, A, Thomas, R, \& Sackmann, E. (1991) Structure of an adsorbed dimyristoylphosphatidylcholine bilayer measured with specular reflection of neutrons. Biophysical Journal 59, 289-294.

[137] Hetzer, M, Heinz, S, Grage, S, \& Bayerl, T. (1998) Asymmetric molecular friction in supported phospholipid bilayers revealed by nmr measurements of lipid diffusion. Langmuir 14, 982-984.

[138] Przybylo, M, Sỳkora, J, Humpolíc ková, J, Benda, A, Zan, A, \& Hof, M. (2006) Lipid diffusion in giant unilamellar vesicles is more than 2 times faster than in supported phospholipid bilayers under identical conditions. Langmuir 22, 9096-9099.

[139] Guo, L, Har, J. Y, Sankaran, J, Hong, Y, Kannan, B, \& Wohland, T. (2008) Molecular diffusion measurement in lipid bilayers over wide concentration ranges: a comparative study. ChemPhysChem 9, 721-728.

[140] Golebiewska, U, Nyako, M, Woturski, W, Zaitseva, I, \& McLaughlin, S. (2008) Diffusion coefficient of fluorescent phosphatidylinositol 4,5-bisphosphate in the plasma membrane of cells. Molecular biology of the cell 19, 1663-1669.

[141] Golebiewska, U, Gambhir, A, Hangyás-Mihályné, G, Zaitseva, I, Rädler, J, \& McLaughlin, S. (2006) Membrane-bound basic peptides sequester multivalent (PIP2), but not monovalent (PS), acidic lipids. Biophysical Journal 91, 588-599.

[142] Bretscher, A. (1983) Purification of an 80,ooo-dalton protein that is a component of the isolated microvillus cytoskeleton, and its localization in nonmuscle cells. Journal of Cell Biology 97, 425-432. 
[143] Lankes, W, Griesmacher, A, Grünwald, J, Schwartz-Albiez, R, \& Keller, R. (1988) A heparin-binding protein involved in inhibition of smooth-muscle cell proliferation. Biochemical Journal 251, 831.

[144] Tsukita, S, Hieda, Y, \& Tsukita, S. (1989) A new 82-kD barbed end-capping protein (radixin) localized in the cell-to-cell adherens junction: purification and characterization. Journal of Cell Biology 108, 2369-2382.

[145] Sato, N, Funayama, N, Nagafuchi, A, Yonemura, S, \& Tsukita, S. (1992) A gene family consisting of ezrin, radixin and moesin. its specific localization at actin filament/plasma membrane association sites. Journal of Cell Science 103, 131-143.

[146] Franck, Z, Gary, R, \& Bretscher, A. (1993) Moesin, like ezrin, colocalizes with actin in the cortical cytoskeleton in cultured cells, but its expression is more variable. Journal of Cell Science 105, 219-231.

[147] Amieva, M. R \& Furthmayr, H. (1995) Subcellular localization of moesin in dynamic filopodia, retraction fibers, and other structures involved in substrate exploration, attachment, and cell-cell contacts. Experimental Cell Research 219, 180-196.

[148] Berryman, M, Franck, Z, \& Bretscher, A. (1993) Ezrin is concentrated in the apical microvilli of a wide variety of epithelial cells whereas moesin is found primarily in endothelial cells. Journal of Cell Science 105, 1025-1043.

[149] Amieva, M. (1994) Radixin is a component of hepatocyte microvilli in situ. Experimental Cell Research 210, 140-144.

[150] Kitajiri, S.-i, Fukumoto, K, Hata, M, Sasaki, H, Katsuno, T, Nakagawa, T, Ito, J, Tsukita, S, \& Tsukita, S. (2004) Radixin deficiency causes deafness associated with progressive degeneration of cochlear stereocilia. Journal of Cell Biology 166, 559-570.

[151] Bretscher, A, Reczek, D, \& Berryman, M. (1997) Ezrin: a protein requiring conformational activation to link microfilaments to the plasma membrane in the assembly of cell surface structures. Journal of Cell Science 110, 3011-3018.

[152] Chishti, A. H, Kim, A. C, Marfatia, S. M, Lutchman, M, Hanspal, M, Jindal, H, Liu, S.-C, Low, P. S, Rouleau, G. A, Mohandas, N, et al. (1998) The FERM domain: a unique module involved in the linkage of cytoplasmic proteins to the membrane. Trends in Biochemical Sciences 23, 281.

[153] Hamada, K, Shimizu, T, Matsui, T, Tsukita, S, Tsukita, S, \& Hakoshima, T. (2000) Structural basis of the membrane-targeting and unmasking mechanisms of the radixin FERM domain. EMBO Journal 19, 4449-4462. 
[154] Smith, W. J, Nassar, N, Bretscher, A, Cerione, R. A, \& Karplus, P. A. (2003) Structure of the active N-terminal domain of ezrin: Conformational and mobility changes identify keystone interactions. Journal of Biological Chemistry 278, 4949-4956.

[155] Pearson, M. A, Reczek, D, Bretscher, A, \& Karplus, P. A. (2000) Structure of the ERM protein moesin reveals the FERM domain fold masked by an extended actin binding tail domain. Cell 101, 259-270.

[156] Li, Q, Nance, M. R, Kulikauskas, R, Nyberg, K, Fehon, R, Karplus, P. A, Bretscher, A, \& Tesmer, J. J. (2007) Self-masking in an intact ERM-merlin protein: an active role for the central $\alpha$-helical domain. Journal of Molecular Biology 365, 1446-1459.

[157] Algrain, M, Turunen, O, Vaheri, A, Louvard, D, \& Arpin, M. (1993) Ezrin contains cytoskeleton and membrane binding domains accounting for its proposed role as a membrane-cytoskeletal linker. Journal of Cell Biology 120, 129139.

[158] Hirao, M, Sato, N, Kondo, T, Yonemura, S, Monden, M, Sasaki, T, Takai, Y, \& Tsukita, S. (1996) Regulation mechanism of ERM (ezrin/radixin/moesin) protein/plasma membrane association: possible involvement of phosphatidylinositol turnover and Rho-dependent signaling pathway. Journal of Cell Biology 135, 37-51.

[159] Nakamura, F, Huang, L, Pestonjamasp, K, Luna, E. J, \& Furthmayr, H. (1999) Regulation of F-actin binding to platelet moesin in vitro by both phosphorylation of threonine 558 and polyphosphatidylinositides. Molecular biology of the cell 10, 2669-2685.

[160] Barret, C, Roy, C, Montcourrier, P, Mangeat, P, \& Niggli, V. (2000) Mutagenesis of the phosphatidylinositol 4,5-bisphosphate ( $\mathrm{PIP}_{2}$ ) binding site in the $\mathrm{NH}$ 2-terminal domain of ezrin correlates with its altered cellular distribution. Journal of Cell Biology 151, 1067-1080.

[161] Maniti, O, Khalifat, N, Goggia, K, Dalonneau, F, Guérin, C, Blanchoin, L, Ramos, L, \& Picart, C. (2012) Binding of moesin and ezrin to membranes containing phophatidylinositol $(4,5)$ bisphosphate: a comparative study of the affinity constants and conformational changes. Biochimica et Biophysica Acta, Biomembranes.

[162] Gautreau, A, Louvard, D, \& Arpin, M. (2000) Morphogenic effects of ezrin require a phosphorylation-induced transition from oligomers to monomers at the plasma membrane. Journal of Cell Biology 150, 193-204. 
[163] Zhou, R, Zhu, L, Kodani, A, Hauser, P, Yao, X, \& Forte, J. G. (2005) Phosphorylation of ezrin on threonine 567 produces a change in secretory phenotype and repolarizes the gastric parietal cell. Journal of Cell Science 118, 4381-4391.

[164] Zhu, L, Zhou, R, Mettler, S, Wu, T, Abbas, A, Delaney, J, \& Forte, J. G. (2007) High turnover of ezrin $\mathrm{T}_{5} 67$ phosphorylation: conformation, activity, and cellular function. American Journal of Physiology: Cell Physiology 293, C874C884.

[165] Gould, K. L, Bretscher, A, Esch, F. S, \& Hunter, T. (1989) cDNA cloning and sequencing of the protein-tyrosine kinase substrate, ezrin, reveals homology to band 4.1. EMBO Journal 8, 4133 .

[166] Herrig, A. (2007) Ph.D. thesis (University of Regensburg).

[167] Leonenko, Z, Finot, E, Ma, H, Dahms, T, \& Cramb, D. (2004) Investigation of temperature-induced phase transitions in DOPC and DPPC phospholipid bilayers using temperature-controlled scanning force microscopy. Biophysical Journal 86, 3783.

[168] Garcia-Manyes, S, Oncins, G, \& Sanz, F. (2005) Effect of temperature on the nanomechanics of lipid bilayers studied by force spectroscopy. Biophysical Journal 89, 4261-4274.

[169] Nissen, J, Gritsch, S, Wiegand, G, \& Rädler, J. (1999) Wetting of phospholipid membranes on hydrophilic surfaces-concepts towards self-healing membranes. European Physical Journal B: Condensed Matter and Complex Systems 10, 335-344.

[170] Gerdes, B. (2013) Master's thesis (Georg-August University Göttingen).

[171] Hanson, R. M. (2010) Jmol-a paradigm shift in crystallographic visualization. Journal of Applied Crystallography 43, 1250-1260.

[172] Menke, M, Ross, M, Gerke, V, \& Steinem, C. (2004) The molecular arrangement of membrane-bound annexin A2-S10oA1o tetramer as revealed by scanning force microscopy. ChemBioChem 5, 1003-1006.

[173] Chambers, D. N \& Bretscher, A. (2005) Ezrin mutants affecting dimerization and activation. Biochemistry 44, 3926-3932.

[174] Jayasundar, J. J, Ju, J. H, He, L, Liu, D, Meilleur, F, Zhao, J, Callaway, D. J, \& Bu, Z. (2012) Open conformation of ezrin bound to phosphatidylinositol 4,5-bisphosphate and to F-actin revealed by neutron scattering. Journal of Biological Chemistry 287, 37119-37133. 
[175] Huang, L, Wong, T. Y, Lin, R. C, \& Furthmayr, H. (1999) Replacement of threonine 558, a critical site of phosphorylation of moesin in vivo, with aspartate activates F-actin binding of moesin: Regulation by conformational change. Journal of Biological Chemistry 274, 12803-12810.

[176] Carvalho, K, Khalifat, N, Maniti, O, Nicolas, C, Arold, S, Picart, C, \& Ramos, L. (2010) Phosphatidylinositol 4, 5-bisphosphate-induced conformational change of ezrin and formation of ezrin oligomers. Biochemistry 49, 9318-9327.

[177] Berryman, M, Gary, R, \& Bretscher, A. (1995) Ezrin oligomers are major cytoskeletal components of placental microvilli: a proposal for their involvement in cortical morphogenesis. Journal of Cell Biology 131, 1231-1242.

[178] Ishikawa, H, Tamura, A, Matsui, T, Sasaki, H, Hakoshima, T, Tsukita, S, \& Tsukita, S. (2001) Structural conversion between open and closed forms of radixin: low-angle shadowing electron microscopy. Journal of Molecular Biology 310, 973-978.

[179] Nagle, J. F \& Tristram-Nagle, S. (2000) Structure of lipid bilayers. Biochimica et Biophysica Acta, Reviews on Biomembranes 1469, 159-195.

[18o] Abollino, O, Aceto, M, Malandrino, M, Sarzanini, C, \& Mentasti, E. (2003) Adsorption of heavy metals on na-montmorillonite. effect of ph and organic substances. Water Research 37, 1619-1627.

[181] Knight, J. D, Lerner, M. G, Marcano-Velázquez, J. G, Pastor, R. W, \& Falke, J. J. (2010) Single molecule diffusion of membrane-bound proteins: window into lipid contacts and bilayer dynamics. Biophysical Journal 99, 2879-2887.

[182] Hansma, H. G \& Hoh, J. H. (1994) Biomolecular imaging with the atomic force microscope. Annual Review of Biophysics and Biomolecular Structure 23, 115-140.

[183] Viani, M. B, Schaffer, T. E, Chand, A, Rief, M, Gaub, H. E, \& Hansma, P. K. (1999) Small cantilevers for force spectroscopy of single molecules. Journal of Applied Physics 86, 2258-2262.

[184] Ando, T, Kodera, N, Takai, E, Maruyama, D, Saito, K, \& Toda, A. (2001) A high-speed atomic force microscope for studying biological macromolecules. Proceedings of the National Academy of Sciences 98, 12468-12472.

[185] Pollard, T. D \& Cooper, J. A. (2009) Actin, a central player in cell shape and movement. Science 326, 1208-1212. 
[186] Neisch, A. L \& Fehon, R. G. (2011) Ezrin, radixin and moesin: key regulators of membrane-cortex interactions and signaling. Current Opinion in Cell Biology 23, 377-382.

[187] Dos Remedios, C, Chhabra, D, Kekic, M, Dedova, I, Tsubakihara, M, Berry, D, \& Nosworthy, N. (2003) Actin binding proteins: regulation of cytoskeletal microfilaments. Physiological Reviews 83, 433-473.

[188] Podolski, J. L \& Steck, T. L. (1990) Length distribution of F-actin in dictyostelium discoideum. Journal of Biological Chemistry 265, 1312-1318.

[189] Holmes, K. C, Popp, D, Gebhard, W, Kabsch, W, et al. (1990) Atomic model of the actin filament. Nature $347,44-49$.

[190] Otterbein, L. R, Graceffa, P, \& Dominguez, R. (2001) The crystal structure of uncomplexed actin in the ADP state. Science 293, 708-711.

[191] Oda, T, Iwasa, M, Aihara, T, Maéda, Y, \& Narita, A. (2009) The nature of the globular-to fibrous-actin transition. Nature $457,441-445$.

[192] Pollard, T. D, Blanchoin, L, \& Mullins, R. D. (2000) Molecular mechanisms controlling actin filament dynamics in nonmuscle cells. Annual Review of Biophysics and Biomolecular Structure 29, 545-576.

[193] Rafelski, S. M \& Theriot, J. A. (2004) Crawling toward a unified model of cell motility: spatial and temporal regulation of actin dynamics. Annual Review of Biochemistry 73, 209-239.

[194] Lodish, H, Berk, A, Matsudaira, P, Kaiser, C. A, Krieger, M, Scott, M. P, Zipursky, L, \& Darnell, J. (2003) Molecular Cell Biology. (W. H. Freeman).

[195] Hoh, J. H, Cleveland, J. P, Prater, C. B, Revel, J. P, \& Hansma, P. K. (1992) Quantized adhesion detected with the atomic force microscope. Journal of the American Chemical Society 114, 4917-4918.

[196] Kramers, H. A. (1940) Brownian motion in a field of force and the diffusion model of chemical reactions. Physica 7, 284-304.

[197] Hänggi, P, Talkner, P, \& Borkovec, M. (1990) Reaction-rate theory: fifty years after Kramers. Reviews of Modern Physics 62, 251.

[198] Bell, G. (1978) Models for the specific adhesion of cells to cells. Science 200, 618-627.

[199] Zhurkov, S. N. (1965) Kinetic concept of the strength of solids. International Journal of Fracture Mechanics 1, 311-323. 
[200] Evans, E \& Ritchie, K. (1997) Dynamic strength of molecular adhesion bonds. Biophysical Journal 72, 1541 - 1555 .

[201] Evans, E \& Ritchie, K. (1999) Strength of a weak bond connecting flexible polymer chains. Biophysical Journal 76, 2439-2447.

[202] Rief, M, Oesterhelt, F, Heymann, B, \& Gaub, H. (1997) Single molecule force spectroscopy on polysaccharides by atomic force microscopy. Science 275, $1295-1297$.

[203] Oberhauser, A. F, Marszalek, P. E, Erickson, H. P, \& Fernandez, J. M. (1998) The molecular elasticity of the extracellular matrix protein tenascin. Nature $393,181-185$.

[204] Merkel, R, Nassoy, P, Leung, A, Ritchie, K, \& Evans, E. (1999) Energy landscapes of receptor-ligand bonds explored with dynamic force spectroscopy. Nature 397, 50-53.

[205] Strunz, T, Oroszlan, K, Schäfer, R, \& Güntherodt, H.-J. (1999) Dynamic force spectroscopy of single DNA molecules. Proceedings of the National Academy of Sciences 96, 11277-11282.

[206] Baumgartner, W, Hinterdorfer, P, Ness, W, Raab, A, Vestweber, D, Schindler, H, \& Drenckhahn, D. (2000) Cadherin interaction probed by atomic force microscopy. Proceedings of the National Academy of Sciences 97, 4005-4010.

[207] Ducker, W. A, Senden, T. J, \& Pashley, R. M. (1991) Direct measurement of colloidal forces using an atomic force microscope. Nature 353, 239-241.

[208] Butt, H.-J. (1991) Measuring electrostatic, van der Waals, and hydration forces in electrolyte solutions with an atomic force microscope. Biophysical Journal 6o, 1438-1444.

[209] Hertz, H. (1882) Über die Berührung fester elastischer Körper. J. für die reine u. angew. Math. 92.

[210] Lorenz, B, Keller, R, Sunnick, E, Geil, B, \& Janshoff, A. (2010) Colloidal probe microscopy of membrane-membrane interactions: From ligand-receptor recognition to fusion events. Biophysical Chemistry 150, 54-63.

[211] Moens, P. D \& Bagatolli, L. A. (2007) Profilin binding to sub-micellar concentrations of phosphatidylinositol $(4,5)$ bisphosphate and phosphatidylinositol $(3,4,5)$ trisphosphate. Biochimica et Biophysica Acta, Biomembranes 1768, 439449 . 
[212] Huang, F \& Huang, K. (1991) Interaction of protein kinase C isozymes with phosphatidylinositol 4,5-bisphosphate. Journal of Biological Chemistry 266, $8727-8733$.

[213] Cooper, J. (1987) Effects of cytochalasin and phalloidin on actin. Journal of Cell Biology 105, 1473-1478.

[214] Freedman, D \& Diaconis, P. (1981) On the histogram as a density estimator: L 2 theory. Probability Theory and Related Fields 57, 453-476.

[215] Epanechnikov, V. A. (1969) Non-parametric estimation of a multivariate probability density. Theory of Probability $\mathcal{E}$ Its Applications 14, 153-158.

[216] Frigge, M, Hoaglin, D. C, \& Iglewicz, B. (1989) Some implementations of the boxplot. The American Statistician 43, 50-54.

[217] Martinez, W. L \& Martinez, A. R. (2001) Computational statistics handbook with MATLAB. (Chapman and Hall/CRC) Vol. 2.

[218] Roy, C, Martin, M, \& Mangeat, P. (1997) A dual involvement of the aminoterminal domain of ezrin in F-and G-actin binding. Journal of Biological Chemistry 272, 20088-20095.

[219] Bizzarri, A. R \& Cannistraro, S. (2010) The application of atomic force spectroscopy to the study of biological complexes undergoing a biorecognition process. Chemical Society Reviews 39, 734-749.

[220] Verbelen, C, Gruber, H. J, \& Dufrene, Y. F. (2007) The NTA-His(6) bond is strong enough for AFM single-molecular recognition studies. Journal of Molecular Recognition 20, 490-494.

[221] Braunger, J. A. (2009) Master's thesis (Georg-August University Göttingen).

[222] Nakamura, F, Amieva, M. R, Hirota, C, Mizuno, Y, \& Furthmayr, H. (1996) Phosphorylation of ${ }^{558} \mathrm{t}$ of moesin detected by site-specific antibodies in RAW264.7 macrophages. Biochemical and Biophysical Research Communications 226, 650-656.

[223] Hayashi, K, Yonemura, S, Matsui, T, \& Tsukita, S. (1999) Immunofluorescence detection of ezrin/radixin/moesin (ERM) proteins with their carboxylterminal threonine phosphorylated in cultured cells and tissues. Journal of Cell Science 112, 1149-1158.

[224] Yuan, C, Chen, A, Kolb, P, \& Moy, V. T. (2000) Energy landscape of streptavidin-biotin complexes measured by atomic force microscopy. Biochemistry 39, 10219-10223. 
[225] Hinrichsen, E. L, Feder, J, \& Jøssang, T. (1986) Geometry of random sequential adsorption. Journal of Statistical Physics 44, 793-827.

[226] Losic, D, Gooding, J. J, Shapter, J, Hibbert, D, \& Short, K. (2001) The influence of the underlying gold substrate on glucose oxidase electrodes fabricated using self-assembled monolayers. Electroanalysis 13, 1385-1393.

[227] Rabinovich, Y. I, Adler, J. J, Ata, A, Singh, R. K, \& Moudgil, B. M. (2000) Adhesion between nanoscale rough surfaces: II. Measurement and comparison with theory. Journal of Colloid and Interface Science 232, 17-24.

[228] Drelich, J, Tormoen, G. W, \& Beach, E. R. (2004) Determination of solid surface tension from particle-substrate pull-off forces measured with the atomic force microscope. Journal of Colloid and Interface Science 280, 484-497.

[229] Cocco, S, Monasson, R, \& Marko, J. F. (2001) Force and kinetic barriers to unzipping of the dna double helix. Proceedings of the National Academy of Sciences 98, 8608-8613.

[230] Albrecht, C, Blank, K, Lalic-Mülthaler, M, Hirler, S, Mai, T, Gilbert, I, Schiffmann, S, Bayer, T, Clausen-Schaumann, H, \& Gaub, H. E. (2003) DNA: a programmable force sensor. Science 301, 367-370.

[231] Erdmann, T. (2005) Ph.D. thesis (Max Planck Institute of Colloids and Interfaces).

[232] Seifert, U. (2000) Rupture of multiple parallel molecular bonds under dynamic loading. Physical Review Letters 84, 2750-2753.

[233] Lorenz, B, Álvarez de Cienfuegos, L, Oelkers, M, Kriemen, E, Brand, C, Stephan, M, Sunnick, E, Yüksel, D, Kalsani, V, Kumar, K, et al. (2012) Model system for cell adhesion mediated by weak carbohydrate-carbohydrate interactions. Journal of the American Chemical Society 134, 3326-3329.

[234] Friddle, R. W, Noy, A, \& De Yoreo, J. J. (2012) Interpreting the widespread nonlinear force spectra of intermolecular bonds. Proceedings of the National Academy of Sciences 109, 13573-13578.

[235] Kim, B.-H, Palermo, N. Y, Lovas, S, Zaikova, T, Keana, J. F, \& Lyubchenko, Y. L. (2011) Single-molecule atomic force microscopy force spectroscopy study of $\mathrm{A} \beta-40$ interactions. Biochemistry 50, 5154-5162.

[236] Zhang, X, Wojcikiewicz, E, \& Moy, V. T. (2002) Force spectroscopy of the leukocyte function-associated antigen-1/intercellular adhesion molecule- 1 interaction. Biophysical Journal 83, 2270. 
[237] Ptak, A, Gojzewski, H, Kappl, M, \& Butt, H.-J. r. (2010) Quantitative analysis of the interaction between an atomic force microscopy tip and a hydrophobic monolayer. Journal of Physical Chemistry C 114, 21572-21578.

[238] Dudko, O. K, Hummer, G, \& Szabo, A. (2006) Intrinsic rates and activation free energies from single-molecule pulling experiments. Physical Review Letters 96, 108101.

[239] Lee, C.-K, Wang, Y.-M, Huang, L.-S, \& Lin, S. (2007) Atomic force microscopy: determination of unbinding force, off rate and energy barrier for proteinligand interaction. Micron 38, 446-461.

[240] Ferrer, J. M, Lee, H, Chen, J, Pelz, B, Nakamura, F, Kamm, R. D, \& Lang, M. J. (2008) Measuring molecular rupture forces between single actin filaments and actin-binding proteins. Proceedings of the National Academy of Sciences 105, 9221-9226.

[241] Hinterdorfer, P \& Van Oijen, A. (2009) Handbook of single-molecule biophysics. (Springer).

[242] Wong, J, Chilkoti, A, \& Moy, V. T. (1999) Direct force measurements of the streptavidin-biotin interaction. Biomolecular Engineering 16, 45-55. 



\section{DANKSAGUNG}

An erster Stelle danke ich Prof. Dr. Claudia Steinem für ihre Unterstützung und stete Diskussionsbereitschaft. Ich bedanke mich für das Vertrauen, das sie mir entgegengebracht hat, die Freiheit meine Ideen umzusetzen und die vielen lehrreichen Gespräche.

Prof. Dr. Sarah Köster gilt mein Dank für die Übernahme des Korreferats. Dr. Iwan Schaap und ihr danke ich für die angenehme und inspirierende Atmosphäre der regelmäßigen Thesis Committee Meetings.

Prof. Dr. Volker Gerke vom ZMBE in Münster danke ich für die gute Zusammenarbeit und hilfreiche Ratschläge.

Prof. Dr. Burkhard Geil danke ich für die Unterstützung bei der Anwendung verschiedener Modelle zur Dateninterpretation.

Dr. Ingo Mey gebührt großer Dank für die Entwicklung der AFM-Auswertungssoftware und die vielen hilfreichen Diskussionen.

Bei unseren technischen Assistenten Jutta Gerber-Nolte und Michaela Klingebiel bedanke ich mich für ihre tatkräftige Unterstützung im Laboralltag. Unseren Sekretärinnen Marianne Wagener und Melanie Sorhage danke ich für ihre Hilfe in organisatorischen Belangen.

Meinen Bachelor- und Masterstudenten Annika, Corinna und Benni möchte ich ganz besonders danken für ihre engagierte und sorgfältige Arbeit. Es war stets eine Freude mit euch zusammenzuarbeiten. Ihr habt mich bei dieser Arbeit tatkräftig unterstützt!

Für das gewissenhafte Korrekturlesen dieser Arbeit bedanke ich mich bei Corinna, Dany, Ole und Ingo.

Daniel möchte ich für das gemeinsame Durchstehen der erlebten Höhen und Tiefen während des Zusammenschreibens unserer Doktorarbeiten danken! 
Dem gesamten Arbeitskreis Steinem danke ich für die wundervolle Zeit! Die äußerst unterhaltsamen Kaffeepausen mit einem breiten Spektrum an Gesprächsthemen, sowie die schönen Ausflüge, die legendären Messzeiten und Kegelabenteuer haben wesentlichen Anteil daran, dass ich immer sehr gerne zur Arbeit gekommen bin - auch wenn die Experimente mal weniger erfolgreich waren. In diesem Sinne auch einen großen Dank an den Arbeitskreis Janshoff! Ich bin sehr froh, dass ihr nach Göttingen gekommen seid!

Meiner Familie danke ich von ganzem Herzen für ihre uneingeschränkte Unterstützung! Ohne euch wäre das alles hier nicht möglich gewesen.

Schließlich danke ich aus tiefstem Herzen Steffen. Danke, dass du für mich da warst und bist! Ich freue mich auf unsere gemeinsame Zukunft! 